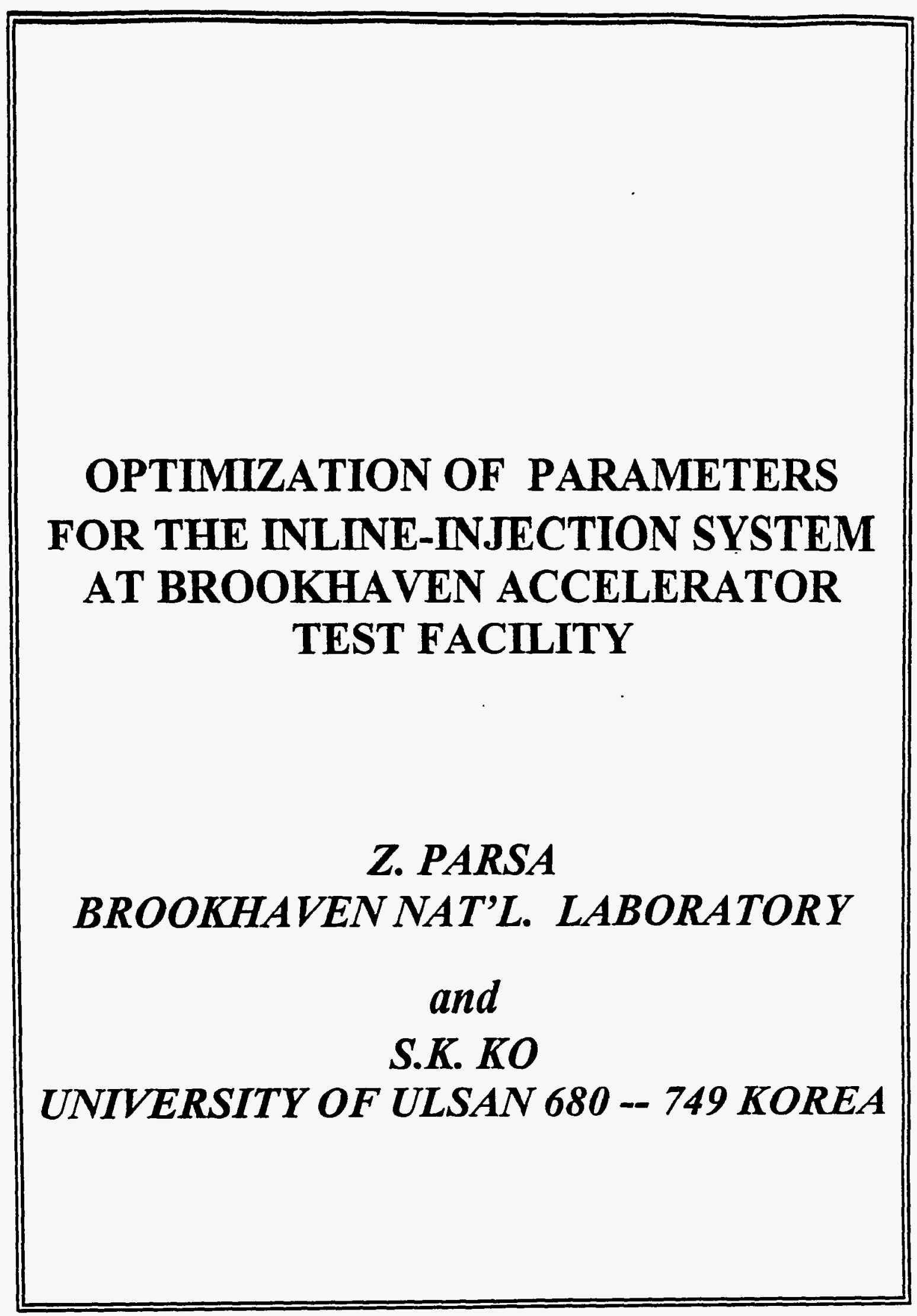




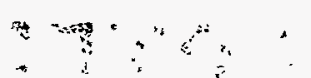

thes 


\section{DISCLAIMER}

This report was prepared as an account of work sponsored by an agency of the United States Government. Neither the United States Government nor any agency thereof, nor any of their employees, make any warranty, express or implied, or assumes any legal liability or responsibility for the accuracy, completeness, or usefulness of any information, apparatus, product, or process disclosed, or represents that its use would not infringe privately owned rights. Reference herein to any specific commercial product, process, or service by trade name, trademark, manufacturer, or otherwise does not necessarily constitute or imply its endorsement, recommendation, or favoring by the United States Government or any agency thereof. The views and opinions of authors expressed herein do not necessarily state or reflect those of the United States Government or any agency thereof. 


\section{DISCLAIMER}

Portions of this document may be illegible in electronic image products. Images are produced from the best available original document. 


\title{
OPTIMIZATION OF PARAMETERS FOR THE INLINE- INJECTION SYSTEM AT BROOKHAVEN ACCELERATOR TEST FACILITY (ATF)
}

\author{
Z. Parsa \\ Brookhaven Nat'l Laboratory \\ Upton, New York 11973-5000
}

and

S. K. Ko

University of Ulsan

680--749 Korea

\begin{abstract}
We present some of our parameter optimization results utilizing code PARMLEA, for the ATF Inline-Injection System. The new solenoid- Gun Solenoid -- Drift-Linac Scheme (Fig. 2) would improve the beam quality needed for FEL and other experiments at ATF as compared to the beam quality of the original design injection system (Fig. 1). To optimize the gain in the beam quality we have considered various parameters including the accelerating field gradient on the photoathode, the Solenoid field strengths, separation between the gun and entrance to the linac as well as the (type and size) initial charge distributions. The effect of the changes in these parameters on the beam emittance is also given.
\end{abstract}

References:

Z. Parsa, Development of the Alternate Entrv Port for the ATE, IEEE 1993, 0-7903-1203-1/93, p. 2723 (1993), and Refs. therein; also Z. Parsa, S.Ko, Optimization of the Photocathode - Linac separation for the ATF Injection System (1993), CAP-ATF TN \#17. 
We present some of our parameter optimization results for the Brookhaven Inline-Injection system, obtained with Program PARMELA (modified version on the UNIX Workstation, which gives slightly different results than the version we regularly have used in collaboration with $\mathrm{L}$. Young. This version is used as a test for comparison with other calculations with this program version at $\mathrm{ATF}$ ).

For convenience in interpreting the beam positions and profiles shown in the Figures, Table 1 shows the labeling of the elements in the beam line utilizing the input format for the code PARMELA.

The change in the beam emittance with variations in the initial particle distributions (with $66 \mathrm{MV} / \mathrm{m}$ field on the cathode ) are shown: In Tables 2-1 to 2-4 (for flat, $1 \sigma, 2 \sigma$, and $3 \sigma$ Guassian distributions), for the case of $I=0$; and in Tables 3-1 to 3-3 (for flat, $1 \sigma$, and $2 \sigma$ Guassian distributions), with solenoid current $\mathrm{I}=2180 \mathrm{amp}$. Figures 1 and 2 gives sketches of the original and new ATF injection systems. The new injection system consists of a solenoid - gun - solenoid inline with the linac, combination which could improve the beam quality needed for FEL and other experiments (compared to the original ATF double bend design (see Fig 1)). In this scheme the 1st solenoid (proceeding the gun) bucks the magnetic field of the 2nd solenoid to assure 0 (no degrading) field on the photocathode. Comparison of these figures provides the effects of the variations in the initial particle distributions and the solenoid field on the beam emittance.

Comparison of the results presented in Tables 2 and 3 (with $66 \mathrm{MV} / \mathrm{m}$ the field gradient on the cathode) to those shown in Figures 3 to 13 (with 100 $\mathrm{MV} / \mathrm{m}$ field gradient on the cathode ) further illustrates, the advantage of applying a higher accelerating gradients at the photocathode (avoiding the beam emittance growth). Figures 3 t0 13 show the variations in the distance between the gun exit and the linac entrance, as well as the effects of the variations in the solenoid field (current) on the beam. In moving from the cathode through the gun the emittance first increases then decreases and diminishes. As the beam drifts out of the gun it accelerates and the beam emittance grows due to space charge (electron bunch self field) defocusing. forces. By using a solenoid we compensate for the space charge defocusing forces. The location of the waist changes with the change in the solenoid field strength, as can be seen in Figures 3 to 13. 
Additional figures and beam profiles for the new injection scheme with other solenoid field strengths as well as the results obtained with various initial distributions were given in our earlier papers ( See Ref.). 


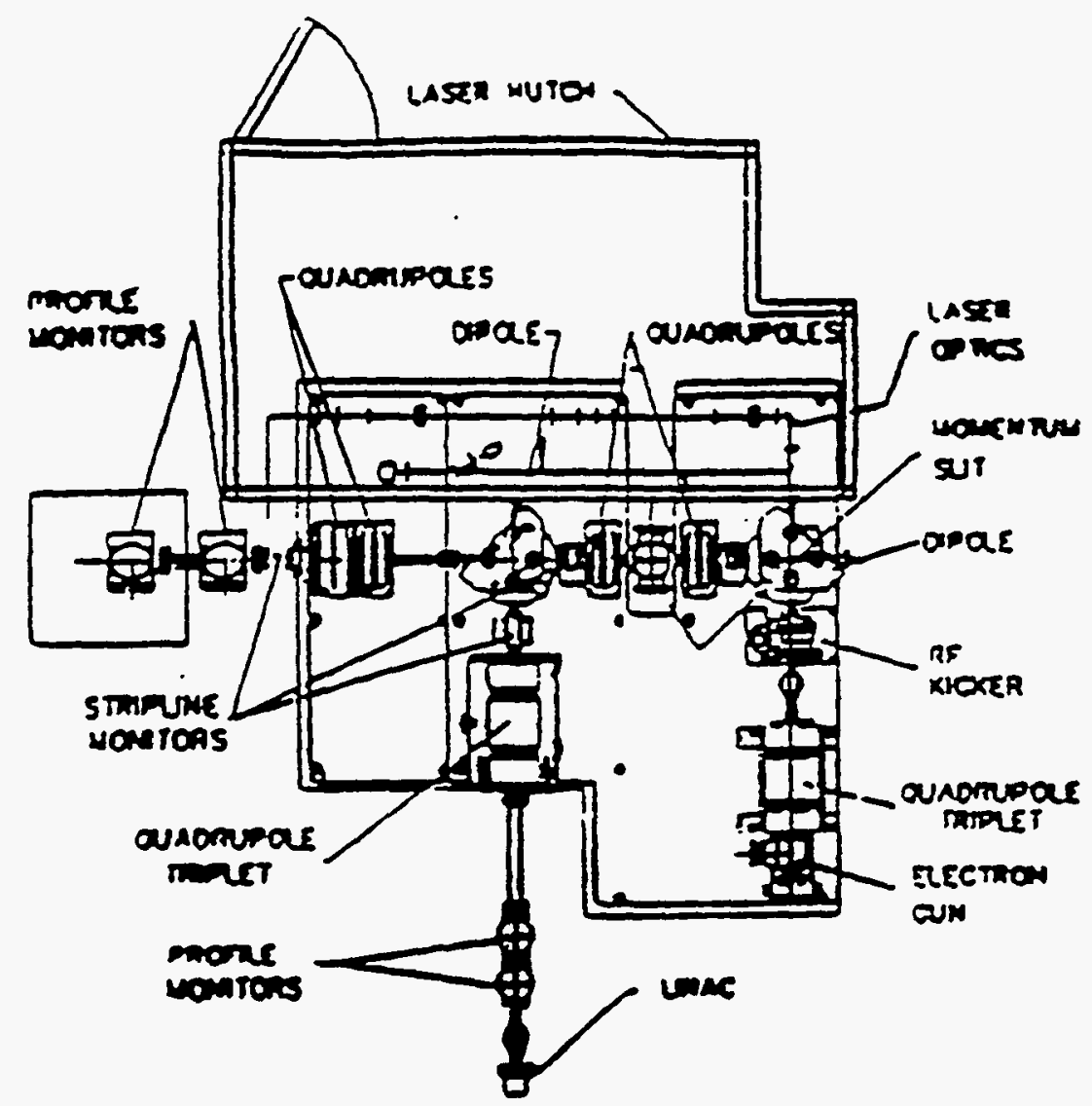

Figure 1. A sketch of the Brookhaven ATF (original design) Injection system with the transport beamline from the gun to the linac entrance. Showing the positions of the magnetic elements.

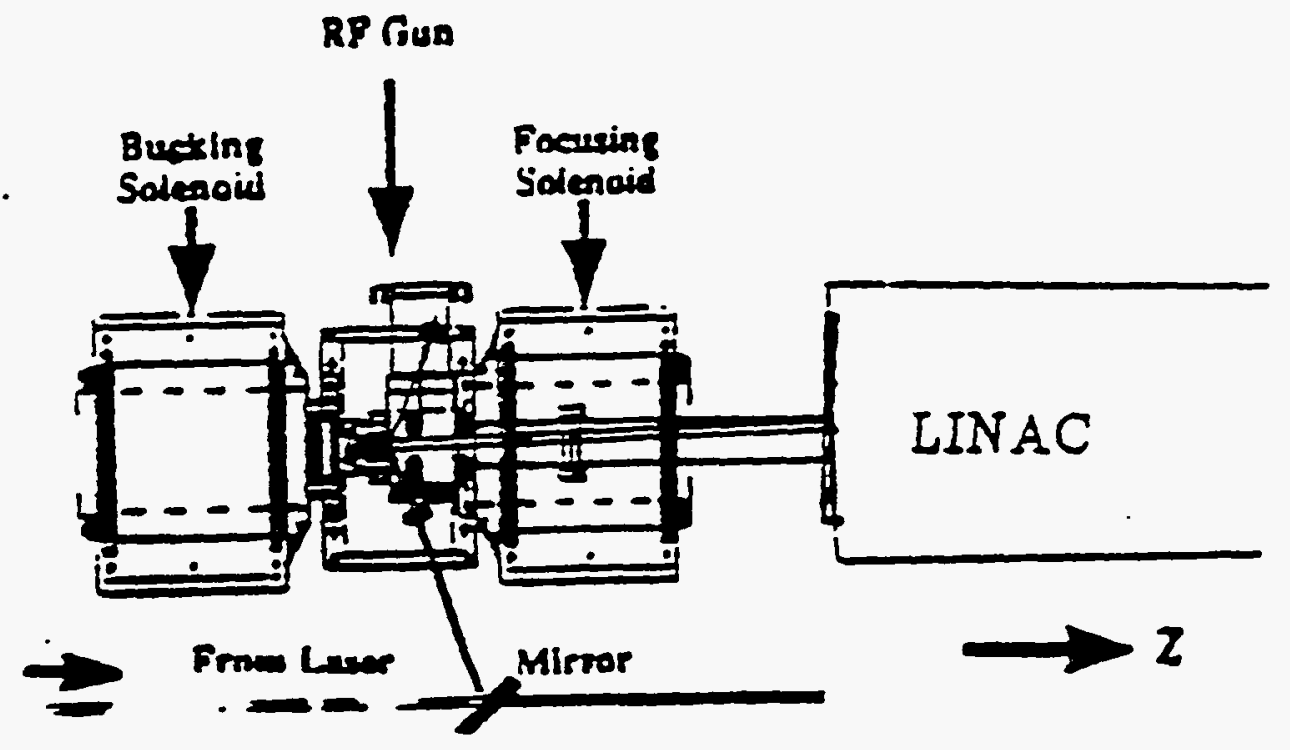

Figure 2. A sketch of the Brookhaven ATF (new design) Inline-Injection system with the Solenoid+Gun+Solenoid combination placed inline with the linac. 
Table 1. Shows the labling of the elements in the beam line (in PARMELA format) from the Cathede (at $z=0$ ), through the RF Gun and at various points (distance $z$ from the cathode) to the Linac entrance.

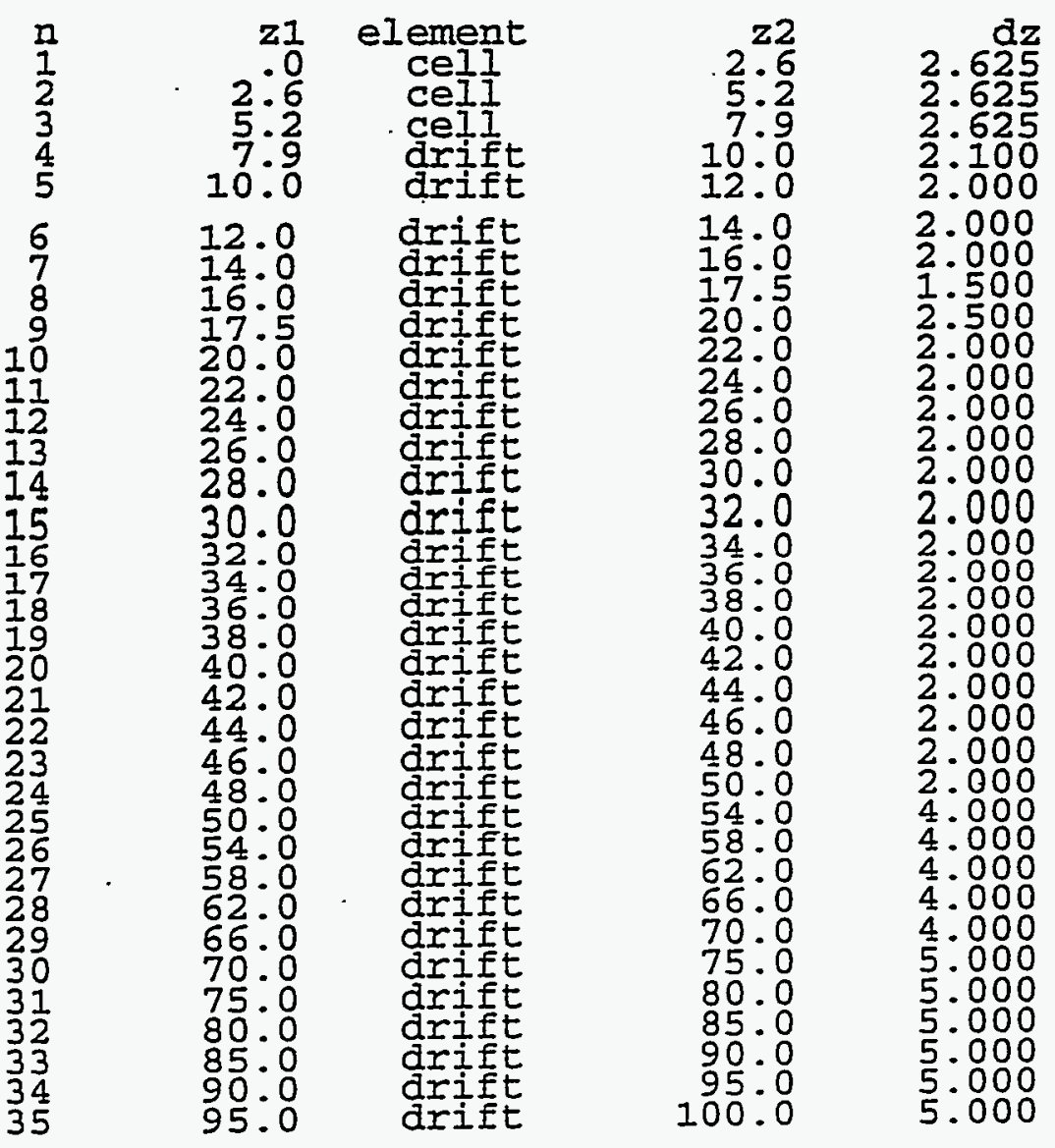




\section{SUMMARY:}

We presented some of our results, for the ATF Inline-Injection System utilizing PARMLEA. As we showed previously, the alternate solenoid - Gun - Solenoid -- Linac Scheme (Fig. 2) would improve the beam quality needed for FEL and other experiments at ATF as compared to the beam quality of the original design injection system (Fig. 1). To optimize the gain in the beam quality we have considered various parameters including the accelerating field gradient on the photocathode, the Solenoid field strengths, separation between the gun and entrance to the linac as well as the (type and size) initial charge distributions. The effects of the changes in these parameters on the beam emittance were shown in Tables 2 and 3, and Figures 3 to 13 .

Additional figures and beam profiles for the new injection scheme with other solenoid field strengths as well as the results obtained with various initial distributions were given in our earlier papers ( See Ref.). With solenoid-Gunsolenoid straight injection into the linac scheme we can reduce the emittance dilution due to space charge forces, and produce the beam needed for FEL, IFEL and other laser acceleration experiments. The effects of variations in the solenoid strengths and $\mathbf{d}$ the distance from the photocathode to the linac entrance was studied and can be detrimental to the quality of beam. Figures 3-13 shows the effects of the variation in $\mathbf{d}$ on the beam profile for various solenoid field strengths. These figures further illustrate how the position of waist changes with the change in initial parameters such as solenoid strength and the distance $\mathrm{d}$ between the cathode and linac. 
BNL RF GUN

$\begin{array}{ccc}\begin{array}{c}\text { nel } \\ \text { no. }\end{array} & \begin{array}{l}\text { part } \\ \text { in }\end{array} & \begin{array}{l}\text { part } \\ \text { out }\end{array} \\ 1 & 6000 & 6000 \\ 2 & 6000 & 6000 \\ 3 & 6000 & 6000 \\ 4 & 6000 & 6000 \\ 5 & 6000 & 6000 \\ 6 & 6000 & 6000 \\ 7 & 6000 & 6000 \\ 8 & 6000 & 6000 \\ 9 & 6000 & 6000 \\ 10 & 6000 & 6000 \\ 11 & 6000 & 6000 \\ 12 & 6000 & 6000 \\ 13 & 6000 & 6000 \\ 14 & 6000 & 6000 \\ 15 & 6000 & 6000 \\ 16 & 6000 & 6000 \\ 17 & 6000 & 6000 \\ 18 & 6000 & 6000 \\ 19 & 6000 & 6000 \\ 20 & 6000 & 6000 \\ 21 & 6000 & 6000 \\ 22 & 6000 & 6000 \\ 23 & 6000 & 6000 \\ 24 & 6000 & 6000 \\ 25 & 6000 & 6000 \\ 26 & 6000 & 6000 \\ 27 & 6000 & 6000 \\ 28 & 6000 & 6000 \\ 29 & 6000 & 5895 \\ 30 & 6000 & .5330 \\ 31 & 6000 & 4706 \\ 32 & 6000 & 4208 \\ 33 & 6000 & 3799 \\ 34 & 6000 & 3457 \\ 35 & 6000 & 3169\end{array}$

$(0.9 \mathrm{~mm}) ; 5.15 \mathrm{ps}, 66.6 \mathrm{MV} / \mathrm{m}, 1 \mathrm{nC} ; \mathrm{phi}=43.0$

$r m s, n$
$\times$
.3710
.4050
.4808
.5263
.5715
.6165
.6624
.6977
.7579
.8074
.8580
.9092
1.9609
1.0135
1.0661
1.1175
1.1719
1.2266
1.2811
1.3344
1.3877
1.4415
1.4961
1.5497
1.6576
1.7649
1.8719
1.9792
2.0844
2.1628
2.1583
2.0861
1.9894
1.9069
1.8229

emax $n$
$\mathrm{x}, 1006$
7.5032
7.4041
8.6621
9.1601
9.6159
10.1563
10.8301
11.3646
12.2366
12.9443
13.6296
14.3166
14.9878
15.6546
16.3453
17.0562
17.7489
18.4627
19.1810
19.9775
20.7226
21.4507
22.1767
22.8821
24.3398
25.7330
27.1455
28.5925
29.4955
28.8258
28.4399
29.5316
30.5949
31.5914
30.0121

$\begin{array}{cr}\text { rms,n } & \text { emax } n \\ y & y .90 \% \\ .3650 & 1.9078 \\ .3984 & 2.0956 \\ .4743 & 2.4764 \\ .5196 & 2.7105 \\ .5644 & 2.9388 \\ .6087 & 3.1546 \\ .6538 & 3.3859 \\ .6884 & 3.5278 \\ .7476 & 3.7812 \\ .7964 & 4.0064 \\ .8463 & 4.2603 \\ .8968 & 4.4993 \\ .9478 & 4.7458 \\ .9996 & 5.0088 \\ 1.0515 & 5.2904 \\ 1.1022 & 5.5351 \\ 1.1559 & 5.8054 \\ 1.2099 & 6.0762 \\ 1.2638 & 6.3577 \\ 1.3163 & 6.6259 \\ 1.3690 & 6.9056 \\ 1.4221 & 7.1752 \\ 1.4761 & 7.4502 \\ 1.5291 & 7.7354 \\ 1.6358 & 8.2977 \\ 1.7420 & 8.8348 \\ 1.8479 & 9.4040 \\ 1.9540 & 9.9536 \\ 2.0478 & 10.5107 \\ 2.0942 & 10.9096 \\ 2.0820 & 10.8515 \\ 2.0343 & 10.5194 \\ 1.9959 & 10.0423 \\ 1.9114 & 9.4774 \\ 1.8466 & 9.2680\end{array}$
rms, $\mathrm{n}$

emax n
$\times, 90 \%$
1.8965
2.0929
2.4656
2.6922
2.9017
3.1329
3.3557
3.5277
3.8219
4.0639
4.2817
4.5001
4.7426
4.9932
5.2466
5.4960
5.7533
6.0180
6.3353
6.6116
6.8800
7.1722
7.4424
7.7007
8.2359
8.7715
9.3085
9.8717
10.5401
11.1152
11.2282
10.6266
10.0923
9.6483
9.2926

Table 2-1. Shows variation of emittance through the gun, at the the gun exit and out side of the gun through the drift space(s) to the Linac entrance. With $66 \mathrm{MV} / \mathrm{m}$ field on the cathode for the Inline-Injection system with $I=0$, and "flat" beam. 
Table 2-2. Shows variation of emittance through the gun, at the the gun exit and out side of the gun through the drift space(s) to the Linac entrance. With $66 \mathrm{MV} / \mathrm{m}$ field on the cathode for the Inline-Injection system with $\underline{I=0}$, "Gaussian distribution, and $1 \sigma$ cut.

BNL RF GUN

\begin{tabular}{|c|c|c|c|c|}
\hline $\begin{array}{l}\text { nel } \\
\text { no. }\end{array}$ & $\operatorname{part}_{\text {in }}$ & part & $\underset{x}{r m s}, n$ & $\operatorname{emax}_{x, 90 \%}^{n}$ \\
\hline $\begin{array}{l}1 \\
2 \\
3 \\
4 \\
5 \\
6 \\
7 \\
8 \\
9 \\
10 \\
11 \\
12 \\
13 \\
14 \\
15 \\
16 \\
17 \\
18 \\
19 \\
20 \\
21 \\
22 \\
23 \\
24 \\
25 \\
26 \\
27 \\
28 \\
29 \\
30 \\
31 \\
32 \\
33 \\
34 \\
35\end{array}$ & $\begin{array}{l}6000 \\
6000 \\
6000 \\
6000 \\
6000 \\
6000 \\
6000 \\
6000 \\
6000 \\
6000 \\
6000 \\
6000 \\
6000 \\
6000 \\
6000 \\
6000 \\
6000 \\
6000 \\
6000 \\
6000 \\
6000 \\
6000 \\
6000 \\
6000 \\
6000 \\
6000 \\
6000 \\
6000 \\
6000 \\
6000 \\
6000 \\
6000 \\
6000 \\
6000 \\
6000\end{array}$ & $\begin{array}{l}6000 \\
6000 \\
6000 \\
6000 \\
6000 \\
6000 \\
6000 \\
6000 \\
6000 \\
6000 \\
6000 \\
6000 \\
6000 \\
6000 \\
6000 \\
6000 \\
6000 \\
6000 \\
6000 \\
6000 \\
6000 \\
6000 \\
6000 \\
6000 \\
6000 \\
6000 \\
6000 \\
5863 \\
5235 \\
4686 \\
4172 \\
3711 \\
3331 \\
2980 \\
2684\end{array}$ & $\begin{array}{r}.4297 \\
.4555 \\
.5573 \\
.6144 \\
.6712 \\
.7291 \\
.7884 \\
.8336 \\
.9099 \\
.9713 \\
1.0329 \\
1.0954 \\
1.1575 \\
1.2205 \\
1.2847 \\
1.3477 \\
1.4116 \\
1.4756 \\
1.5395 \\
1.6033 \\
1.6670 \\
1.7320 \\
1.7962 \\
1.8606 \\
1.9885 \\
2.1169 \\
2.2467 \\
2.3668 \\
2.4330 \\
2.4566 \\
2.4426 \\
2.3976 \\
2.3475 \\
2.2544 \\
2.1890\end{array}$ & $\begin{array}{r}1.9115 \\
2.1142 \\
2.6176 \\
2.9255 \\
3.2174 \\
3.5112 \\
3.8036 \\
4.0231 \\
4.395 \\
4.7226 \\
5.0207 \\
5.3331 \\
5.6314 \\
5.9197 \\
6.2409 \\
6.5310 \\
6.8537 \\
7.1851 \\
7.4847 \\
7.7867 \\
8.1108 \\
8.4443 \\
8.7929 \\
9.1223 \\
9.7496 \\
10.4079 \\
11.0947 \\
11.8152 \\
12.4517 \\
12.7190 \\
12.6033 \\
12.4315 \\
12.1435 \\
11.3968 \\
11.2021\end{array}$ \\
\hline
\end{tabular}

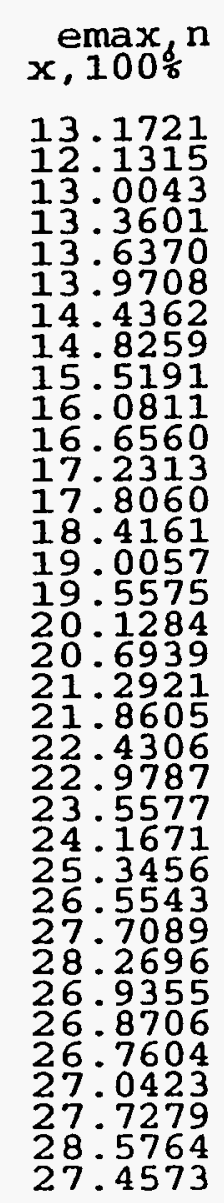

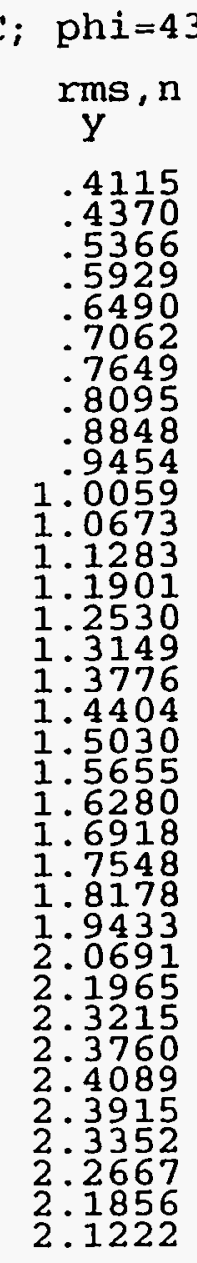

$\operatorname{emax}_{y, 90 \frac{6}{6}} n$

1.8329

2.0238

$2 \cdot 5082$

(1)

3.0890

3.6253

3.6307

4.1821

4.4820

4.7486

5.0230

5.3061

5.8934

6.1961

6.5030

6.8124

7.1312

7.4327

8.7516

8.3596

8.3596

9.6664

9.9260

10.5432

11.2441

11.8080

11.9695

12.1203

11.6767

11.0768

10.7291 emax $n$ $y, 100 \frac{5}{5}$

13.0765

12.5367

12.6595

12.7926

13.0317

13.4598

14.3829

14.8506

15.3234

15.7921

16.2746

16.7505

17.9180

18.5549

19.1883

19.8395

21.0779

21.6900

22.3460

23.0155

24.3067

26.8585

27.6168

26.6530

26.6364

26.8519

27.9292

27.2720

27.7605 
Table 2-3. Shows variation of emittance through the gun, at the the gun exit and out side of the gun through the drift space(s) to the Linac entrance. With $66 \mathrm{MV} / \mathrm{m}$ field on the cathode for the Inline-Injection system with $\underline{I=0}$, "Gaussian distribution, and $2 \sigma$ cut.

BNL RF GUN

$\begin{array}{ccc}\text { nel } & \text { part } & \begin{array}{l}\text { part } \\ \text { no }\end{array} \\ 1 & & \text { out } \\ 1 & 6000 & 6000 \\ 2 & 6000 & 6000 \\ 3 & 6000 & 6000 \\ 4 & 6000 & 6000 \\ 5 & 6000 & 6000 \\ 6 & 6000 & 6000 \\ 7 & 6000 & 6000 \\ 8 & 6000 & 6000 \\ 9 & 6000 & 6000 \\ 10 & 6000 & 6000 \\ 11 & 6000 & 6000 \\ 12 & 6000 & 6000 \\ 13 & 6000 & 6000 \\ 14 & 6000 & 6000 \\ 15 & 6000 & 6000 \\ 16 & 6000 & 6000 \\ 17 & 6000 & 6000 \\ 18 & 6000 & 6000 \\ 19 & 6000 & 6000 \\ 20 & 6000 & 6000 \\ 21 & 6000 & 6000 \\ 22 & 6000 & 6000 \\ 23 & 6000 & 6000 \\ 24 & 6000 & 6000 \\ 25 & 6000 & 6000 \\ 26 & 6000 & 6000 \\ 27 & 6000 & 6000 \\ 28 & 6000 & 6000 \\ 29 & 6000 & 6000 \\ 30 & 6000 & 6000 \\ 31 & 6000 & 6000 \\ 32 & 6000 & 5580 \\ 33 & 6000 & 4954 \\ 34 & 6000 & 4463 \\ 35 & 6000 & 3972 \\ & & \end{array}$

$(0.9 \mathrm{~mm}) ; 5.15 \mathrm{ps}, 66.6 \mathrm{MV} / \mathrm{m}, 1 \mathrm{nC} ; \mathrm{phi}=43.0$

\begin{tabular}{|c|c|}
\hline $\operatorname{rms}_{x}, n$ & $\underset{x, 9}{\operatorname{emax}}$ \\
\hline 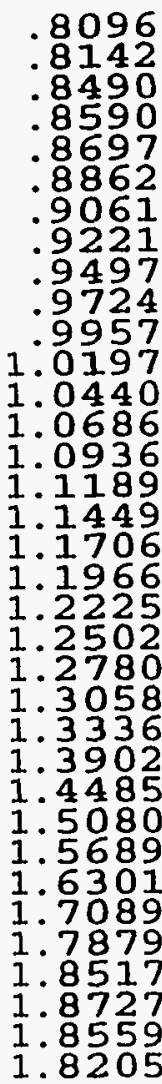 & 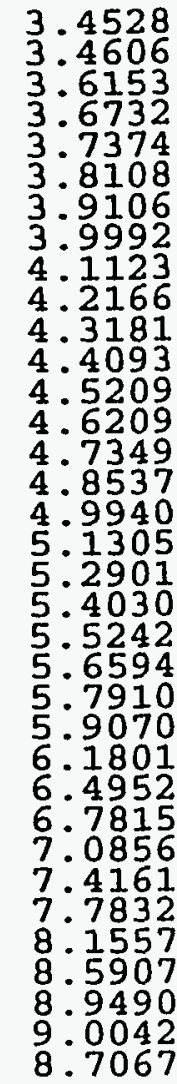 \\
\hline
\end{tabular}

$\operatorname{emax}_{x, 100 \frac{\xi}{6}} n$

38.2458

45.1723

49.8454

53.8403

55.2302

56.3319

56.9971

57.9137

58.9786

59.3224

59.6173

59.8758

60.4144

60.6350

60.8402

61.0281

61.3040

61.4884

61.6672

61.8107

62.0054

62.3460

63.3106

63.6085

63.9668

64.4552

64.8898

62.7116

58.6590

54.0812 rms, $\mathrm{n}$

$$
y
$$

.8050

.8091

.8422

.8631

.8798

.9005

.9461

.9461

.9943

1.0192

1.0444

1.0700

1.0959

1.1488

1.1754

1. 2022

1.2289

1.2576

1.2862

1.3149

1.3436

1.4019

1.5232

1.5857

1.6484

1.7292

1.8103

1.8734

1.8903

1.8549 emax $\mathrm{n}$

$y, 90 \frac{8}{6}$

3.3517
3.3231

3.4231

3.5209

3.5522

3.7738

3.8654

3.9987

4.2339

4.4616

4.4616

4.6680

4.7853

4.9220

5.0198

5.3442

5.4986

5.6518

5.8066

$5 \cdot 9384$

6.4928

6.7619

7.0629

7.3680

8.1613

8.6010

8.9199

9.1118 emax $n$ $y, 100 \frac{8}{6}$

43.6499

55.8061

57.8025

59.5785

60.9412

62.4327

62.9159

63.2252

63.7031

63.9169

64.1298

64.2612

64.6648

64.8026

64.9364

65.0443

65.2787

65.4509

65.5471

65.7735

66.3304

66.5155

66.7559

67.5116

65.2338

61.1833

56.1183 
Table 2-4. Shows variation of emittance through the gun, at the the gun exit and out side of the gun through the drift space(s) to the Linac entrance. With $66 \mathrm{MV} / \mathrm{m}$ field on the cathode for the Inline-Injection system with $\underline{I=0}$, "Gaussian distribution, and $3 \sigma$ cut.

BNL RF GUN

\begin{tabular}{|c|c|c|}
\hline no. & part & ut \\
\hline $\begin{array}{l}1 \\
2 \\
3 \\
4 \\
5 \\
6 \\
7 \\
8 \\
9 \\
10 \\
11 \\
12 \\
13 \\
14 \\
15 \\
16 \\
17 \\
18 \\
19 \\
20 \\
21 \\
22 \\
23 \\
24 \\
25 \\
26 \\
27 \\
28 \\
29 \\
30 \\
31 \\
32\end{array}$ & $\begin{array}{l}6000 \\
6000 \\
6000 \\
6000 \\
6000 \\
000 \\
6000 \\
6000 \\
6000 \\
6000 \\
6000 \\
6000 \\
6000 \\
6000 \\
6000 \\
6000 \\
6000 \\
6000 \\
6000 \\
6000 \\
6000 \\
6000 \\
6000 \\
6000 \\
6000 \\
6000 \\
6000 \\
6000 \\
6000 \\
6000 \\
6000 \\
6000 \\
6000\end{array}$ & $\begin{array}{l}6000 \\
6000 \\
6000 \\
6000 \\
6000 \\
6000 \\
6000 \\
6000 \\
6000 \\
6000 \\
6000 \\
6000 \\
6000 \\
6000 \\
6000 \\
6000 \\
6000 \\
6000 \\
6000 \\
6000 \\
6000 \\
6000 \\
6000 \\
6000 \\
6000 \\
6000 \\
6000 \\
6000 \\
6000 \\
6000 \\
6000 \\
5925 \\
5208 \\
4691\end{array}$ \\
\hline
\end{tabular}

$(0.9 \mathrm{~mm}) ; 5.15 \mathrm{ps}, 66.6 \mathrm{MV} / \mathrm{m}, 1 \mathrm{nC} ; \mathrm{phi}=43.0$

$\begin{array}{lc}\begin{array}{r}r m s, n \\ x\end{array} & \begin{array}{c}\text { emax } \\ x, 96 \%\end{array} \\ .9106 & 3.7444 \\ .9255 & 3.7498 \\ .9620 & 3.9205 \\ .9715 & 3.9871 \\ .9808 & 4.0357 \\ .9948 & 4.1246 \\ 1.0125 & 4.2150 \\ 1.0266 & 4.2963 \\ 1.0508 & 4.4363 \\ 1.0705 & 4.5469 \\ 1.0900 & 4.6330 \\ 1.1097 & 4.7096 \\ 1.1297 & 4.7911 \\ 1.1499 & 4.8802 \\ 1.1701 & 4.9715 \\ 1.1907 & 5.0739 \\ 1.2122 & 5.1738 \\ 1.2338 & 5.2723 \\ 1.2555 & 5.3743 \\ 1.2782 & 5.4746 \\ 1.3007 & 5.5932 \\ 1.3231 & 5.6955 \\ 1.3462 & 5.8348 \\ 1.3698 & 5.9768 \\ 1.4174 & 6.2132 \\ 1.4659 & 6.4256 \\ 1.5173 & 6.6821 \\ 1.5692 & 6.9525 \\ 1.6222 & 7.2110 \\ 1.6895 & 7.5453 \\ 1.7578 & 7.9185 \\ 1.7981 & 8.1621 \\ 1.8177 & 8.4874 \\ 1.8064 & 8.6253 \\ 1.7670 & 8.5938 \\ & \end{array}$

rms, $n$$$
y
$$

.9289

.9434

.9818

.9917

1.0016

1.0164

1.0500

1.0758

1.0968

1.1177

1.1388

1.1600

1. 1814

1.2028

1.2246

1.2471

1.2925

1.3163

1.3399

1.3633

1. 3874

1.4120

1.4617

1.5122

1.5656

1.6795

1.7442

1.8149

1.8611

1.8918

1.9008 emax $n$ y, 90\%

3.6943

3.9635

4.0132

4.0770

4.2516

4.3403

4.4821

4.5710

4.6576

4.7460

4.8367

4.9238

5.0089

5.0959

5.247

5.4637

5.5710

5.6802

5.7844

5.8966

6.0144

6.2984

6.5921

6.8563

7.384

7.7017

8.0660

8.2847

8.5587

8.7584 $\operatorname{emax}_{y, 100 \frac{1}{\delta}} n$

54.2099 57.1810 62.5888 63.2662 64.0481 64.3489 64.4801 66.2711 67.3943 68.3176 69.0770 70.0170 70.9200 71.5309 72.1284 72.7474 73.3091 73.8901 74.2876 74.6815 75.0799 75.4670 $75: 9072$ 76.6742 77.9768 78.5645 79.1213 79.8535 80.5240 82.1138 76.6298 73.0376 
Table 3-1. Shows variation of emittance through the gun, at the the gun exit and out side of the gun through the drift space(s) to the Linac entrance. With $66 \mathrm{MV} / \mathrm{m}$ field on the cathode for the Inline-Injection system, solenoid current of $\underline{I=2180}$, with a "flat" beam.

BNL RF GUN

nel
no.
1
2
3
4
5
6
7
8
9
10
11
12
13
14
15
16
17
18
19
20
21
22
23
24
25
26
27
28
29
30
31
32
33
34
35

part
in
6000
6000
6000
6000
6000
6000
6000
6000
6000
6000
6000
6000
6000
6000
6000
6000
6000
6000
6000
6000
6000
6000
6000
6000
6000
6000
6000
6000
6000
6000
6000
6000
6000
6000
6000
6

$(0.9 \mathrm{~mm}) ; 5$

$\begin{array}{ll}\text { part } & \text { rms, } \\ \text { out } & \mathrm{x} \\ 6000 & .2452 \\ 6000 & .3336 \\ 6000 & .2232 \\ 6000 & .2242 \\ 6000 & .2262 \\ 6000 & .2302 \\ 6000 & .2350 \\ 6000 & .2394 \\ 6000 & .2455 \\ 6000 & .2491 \\ 6000 & .2530 \\ 6000 & .2571 \\ 6000 & .2602 \\ 6000 & .2613 \\ 6000 & .2611 \\ 6000 & .2611 \\ 6000 & .2608 \\ 6000 & .2603 \\ 6000 & .2597 \\ 6000 & .2589 \\ 6000 & .2582 \\ 6000 & .2572 \\ 6000 & .2561 \\ 6000 & .2547 \\ 6000 & .2517 \\ 6000 & .2479 \\ 6000 & .2436 \\ 6000 & .2382 \\ 6000 & .2321 \\ 6000 & .2228 \\ 6000 & .2122 \\ 6000 & .1997 \\ 6000 & .1853 \\ 6000 & .1699 \\ 6000 & .1542\end{array}$

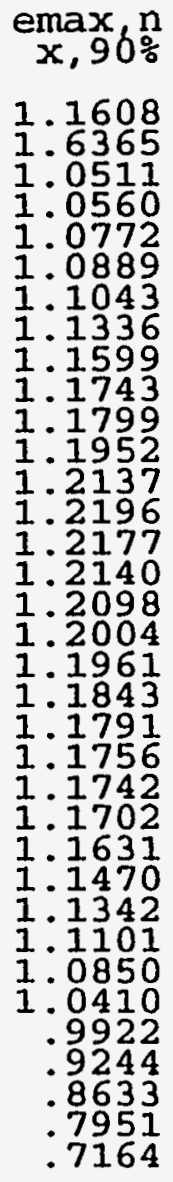

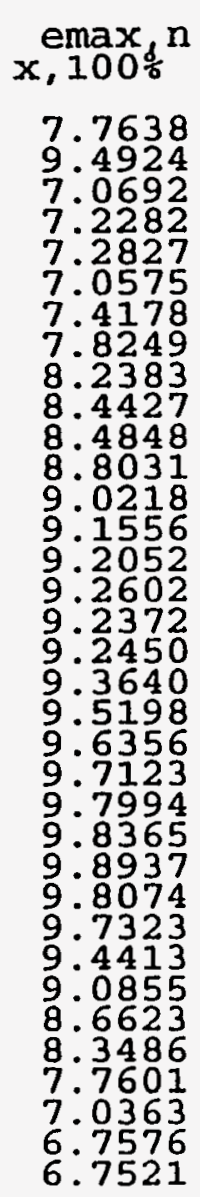

$\mathrm{ph} i=43.0$

$\underset{y}{r m s, n} \quad \operatorname{emax}_{y, 90 \%} n$

$\mathrm{Y}$

.2397

.3261

.2191

.2208

.2243

.2292

.2414

.2508

.2578

.2634

2685

.

. 2698

.2703

.2700

.2696

.2690

.2683

.2676

.2666

.2655

.2612

.2532

.2480

.2419

.2327

.2220

- 1951

.1637
1.1407

1.6213

1.0351

1.0461

1.0890

1.1193

1.1518

1.1861

1.2041

1.2275

1.2371

1.2426

1. 2449

1.2480

1.2533

1.2518

1.2426

1.2359

1. 2352

1.2355

1.2269

1.2084

1. 1893

1.1722

1.1499

1.1251

1. 0266

.9673

.9053

.8301 $\max _{Y, 100 \frac{6}{6}} n$

8.2648 7.2727 7.2868 7.2565 7.1350 7.2931 7.3818

8.1966

8.0961

9.2181

9.5545 9.7918 10.1634 10.2911 10.3640 10.4337 10.4989 10.5085 10.4689 10.4022 10.2375 10.0170 9.8420 9.6529 9.5024

9.1275

8.5563

7.3390

7.4200 
Table 3-2. Shows variation of emittance through the gun, at the the gun exit and out side of the gun through the drift space(s) to the Linac entrance. With $66 \mathrm{MV} / \mathrm{m}$ field on the cathode for the Inline-Injection system, solenoid current of $\underline{I=2180}$, with a "Gaussian distribution, and 1 ocut.

BNL RF GUN $(\mathrm{R}=.04$

\begin{tabular}{|c|c|c|c|}
\hline $\begin{array}{l}\text { nel } \\
\text { no. }\end{array}$ & $\underset{\text { in }}{\text { part }}$ & $\begin{array}{l}\text { part } \\
\text { out }\end{array}$ & $\operatorname{Ims}_{x}, n$ \\
\hline $\begin{array}{r}1 \\
2 \\
3 \\
4 \\
5 \\
6 \\
7 \\
8 \\
9 \\
10 \\
11 \\
12 \\
13 \\
14 \\
15 \\
16 \\
17 \\
18 \\
19 \\
20 \\
21 \\
22 \\
23 \\
24 \\
25 \\
26 \\
27 \\
28 \\
29 \\
30 \\
31 \\
32 \\
33 \\
34 \\
35\end{array}$ & $\begin{array}{l}6000 \\
6000 \\
6000 \\
6000 \\
6000 \\
6000 \\
6000 \\
6000 \\
6000 \\
6000 \\
6000 \\
6000 \\
6000 \\
6000 \\
6000 \\
6000 \\
6000 \\
6000 \\
6000 \\
6000 \\
6000 \\
6000 \\
6000 \\
6000 \\
6000 \\
6000 \\
6000 \\
6000 \\
6000 \\
6000 \\
6000 \\
6000 \\
6000 \\
6000 \\
6000\end{array}$ & $\begin{array}{l}6000 \\
6000 \\
6000 \\
6000 \\
6000 \\
6000 \\
6000 \\
6000 \\
6000 \\
6000 \\
6000 \\
6000 \\
6000 \\
6000 \\
6000 \\
6000 \\
6000 \\
6000 \\
6000 \\
6000 \\
6000 \\
6000 \\
6000 \\
6000 \\
6000 \\
6000 \\
6000 \\
6000 \\
6000 \\
6000 \\
6000 \\
6000 \\
6000 \\
6000 \\
6000\end{array}$ & $\begin{array}{r}.3572 \\
.4123 \\
.3446 \\
.3460 \\
.3479 \\
.3513 \\
.3557 \\
.3597 \\
.3657 \\
.3696 \\
.3729 \\
.3745 \\
.3752 \\
.3746 \\
.3733 \\
.3719 \\
.3703 \\
.3682 \\
.3656 \\
.3625 \\
.3591 \\
.3556 \\
.3520 \\
.3483 \\
.3406 \\
.33244 \\
.3236 \\
.3143 \\
.3042 \\
.2904 \\
.2755 \\
.2592 \\
.2421 \\
.2243 \\
.2062\end{array}$ \\
\hline
\end{tabular}

$(0.9 \mathrm{~mm}) ; 5.15 \mathrm{ps}, 66.6 \mathrm{MV} / \mathrm{m}, 1 \mathrm{nC} ; \mathrm{phi}=43.0$ :'

\begin{tabular}{|c|c|c|c|c|}
\hline $\operatorname{emax}_{x, 96 \frac{0}{6}}$ & $\operatorname{emax}_{x, 100 \frac{f}{6}} n$ & $\underset{y}{\mathrm{rms}}, \mathrm{n}$ & $\operatorname{emax}_{y, 90 \frac{\delta}{6}} n$ & $\operatorname{y.max}_{y, 100 \frac{d}{6}} n$ \\
\hline $\begin{array}{r}1.5017 \\
1.7307 \\
1.4639 \\
1.4663 \\
1.4832 \\
1.4958 \\
1.4996 \\
1.5240 \\
1.5525 \\
1.5708 \\
1.5942 \\
1.6126 \\
1.6284 \\
1.6308 \\
1.6327 \\
1.6356 \\
1.6309 \\
1.6241 \\
1.6118 \\
1.5960 \\
1.5825 \\
1.5659 \\
1.5503 \\
1.5375 \\
1.5083 \\
1.4704 \\
1.4403 \\
1.3943 \\
1.3549 \\
1.2890 \\
1.2176 \\
1.1458 \\
1.0723 \\
.9902 \\
.9025\end{array}$ & $\begin{array}{l}14.4280 \\
16.4855 \\
12.5882 \\
12.6450 \\
12.3690 \\
11.6009 \\
10.2656 \\
9.4593 \\
11.4778 \\
12.8902 \\
13.7198 \\
14.1186 \\
14.3030 \\
14.4050 \\
14.4906 \\
14.5489 \\
14.6070 \\
14.6974 \\
14.7806 \\
14.8975 \\
15.0211 \\
15.1159 \\
15.2360 \\
15.3487 \\
15.5486 \\
15.7328 \\
15.9472 \\
16.1308 \\
16.3297 \\
16.6044 \\
16.8731 \\
17.2398 \\
17.7165 \\
18.3357 \\
19.1361\end{array}$ & $\begin{array}{l}.3446 \\
.3952 \\
.3314 \\
.3324 \\
.3348 \\
.3390 \\
.3457 \\
.3522 \\
.3631 \\
.3712 \\
.3778 \\
.3821 \\
.3845 \\
.3848 \\
.3838 \\
.3826 \\
.3810 \\
.3791 \\
.3766 \\
.3736 \\
.3703 \\
.3669 \\
.3634 \\
.3599 \\
.3522 \\
.3441 \\
.3354 \\
.3261 \\
.3161 \\
.3022 \\
.2872 \\
.2709 \\
.2534 \\
.2350 \\
.2162\end{array}$ & $\begin{array}{l}1.4707 \\
1.6636 \\
1.3989 \\
1.4087 \\
1.4305 \\
1.4502 \\
1.4769 \\
1.5160 \\
1.5857 \\
1.6425 \\
1.6577 \\
1.6629 \\
1.6842 \\
1.6967 \\
1.6985 \\
1.6909 \\
1.6925 \\
1.6893 \\
1.6792 \\
1.6692 \\
1.6508 \\
1.6332 \\
1.6155 \\
1.5990 \\
1.5704 \\
1.5221 \\
1.4892 \\
1.4474 \\
1.4057 \\
1.3390 \\
1.2764 \\
1.2076 \\
1.1301 \\
1.0527 \\
.9622\end{array}$ & $\begin{array}{l}13.8732 \\
16.2587 \\
12.2198 \\
11.7472 \\
11.7570 \\
11.8923 \\
11.9923 \\
12.1799 \\
12.6562 \\
13.2941 \\
13.4073 \\
13.2785 \\
13.0730 \\
13.0193 \\
13.0111 \\
12.9181 \\
12.8917 \\
13.0784 \\
13.2465 \\
13.4340 \\
13.6119 \\
13.7619 \\
13.9293 \\
14.0833 \\
14.3594 \\
14.6127 \\
14.8654 \\
15.0886 \\
15.2869 \\
15.5131 \\
15.6944 \\
15.9034 \\
16.1705 \\
16.5009 \\
16.9267\end{array}$ \\
\hline
\end{tabular}


Table 3-3. Shows variation of emittance through the gun, at the the gun exit and out side of the gun through the drift space(s) to the Linac entrance. With $66 \mathrm{MV} / \mathrm{m}$ field on the cathode for the Inline-Injection system with solenoid current $I=2180, a$ "Gaussian distribution, and $\underline{2 \sigma}$ cut.

BNL RF GUN

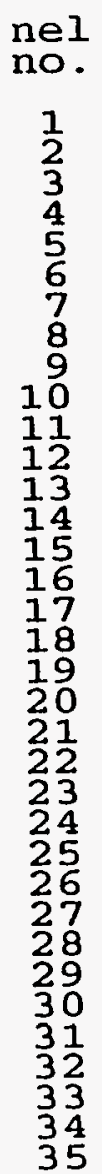

part
in
6000
6000
6000
6000
6000
6000
6000
6000
6000
6000
6000
6000
6000
6000
6000
6000
6000
6000
6000
6000
6000
6000
6000
6000
6000
6000
6000
6000
6000
6000
6000
6000
6000
6000
6000

part

6000

6000

6000

6000

6000

6000

6000

6000

6000

6000

6000

6000

6000

6000

(6)

60

6

6000

6000

6000

6000

6000

6000

6000

6000

6000

6000

600

600

(6)

6000

6000

6000

6000
6000
$(0.9 \mathrm{~mm}) ; 5.15 \mathrm{ps}, 66.6 \mathrm{MV} / \mathrm{m}, 1 \mathrm{nC} ; \mathrm{phi}=43.0$

\begin{tabular}{|c|c|c|c|c|c|}
\hline$\underset{x}{\mathrm{rms}_{\mathrm{m}}}, \mathrm{n}$ & $\underset{x, 90}{\operatorname{emax}}$ & $\underset{x, 100 \frac{8}{6}}{\operatorname{emax}} n$ & $\underset{y}{\mathrm{rms}}, \mathrm{n}$ & $\operatorname{emax}_{y, 90 \frac{\delta}{6}} n$ & $\operatorname{y,100\% }_{\frac{8}{6}}^{\operatorname{emax}}$ \\
\hline $\begin{array}{l}.6968 \\
.7645 \\
.7633 \\
.7726 \\
.7813 \\
.7904 \\
.7996 \\
.8065 \\
.8181 \\
.8272 \\
.8353 \\
.8411 \\
.8452 \\
.8484 \\
.8512 \\
.8537 \\
.8560 \\
.8579 \\
.8595 \\
.8607 \\
.8615 \\
.8620 \\
.8621 \\
.8618 \\
.8600 \\
.8565 \\
.8514 \\
.8446 \\
.8360 \\
.8233 \\
.8091 \\
.7947 \\
.7834 \\
.7775 \\
.7746\end{array}$ & $\begin{array}{l}3.0448 \\
3: 2953 \\
3: 2905 \\
3: 3156 \\
3: 3507 \\
3: 3595 \\
3: 4496 \\
3: 4632 \\
3: 5013 \\
3: 5187 \\
3: 5460 \\
3: 5555 \\
3: 5781 \\
3: 5733 \\
3: 5920 \\
3: 6086 \\
3: 6014 \\
3: 6083 \\
3: 6121 \\
3: 6136 \\
3: 6090 \\
3: 6055 \\
3: 6091 \\
3: 6150 \\
3: 5869 \\
3: 5672 \\
3: 5310 \\
3: 4958 \\
3: 4492 \\
3: 3893 \\
3: 3393 \\
3: 2815 \\
3: 2014 \\
3: 1564 \\
3.1253\end{array}$ & 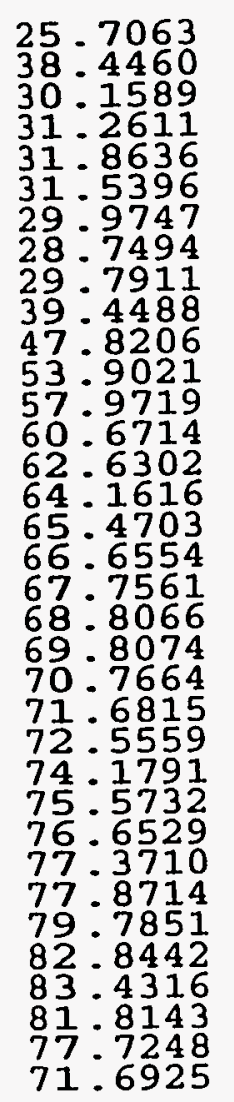 & $\begin{array}{l}.6926 \\
.7626 \\
.7548 \\
.7642 \\
.7739 \\
.7850 \\
.7977 \\
.8080 \\
.8258 \\
.8395 \\
.8510 \\
.8594 \\
.8649 \\
.8690 \\
.8722 \\
.8752 \\
.8778 \\
.8801 \\
.8821 \\
.8837 \\
.8849 \\
.8858 \\
.8862 \\
.8862 \\
.8850 \\
.8820 \\
.8772 \\
.8707 \\
.8621 \\
.8492 \\
.8352 \\
.8221 \\
.8109 \\
.8035 \\
.7984\end{array}$ & $\begin{array}{l}2.9687 \\
3.1897 \\
3.2148 \\
3: 2620 \\
3.3122 \\
3.3674 \\
3.3852 \\
3.4398 \\
3.5114 \\
3: 5752 \\
3.6617 \\
3.6722 \\
3.7020 \\
3: 7285 \\
3: 7595 \\
3: 7859 \\
3.8034 \\
3: 7981 \\
3: 7857 \\
3: 7844 \\
3.7856 \\
3.7742 \\
3.7731 \\
3: 7728 \\
3.7604 \\
3.7368 \\
3.7107 \\
3.6810 \\
3.6390 \\
3.5834 \\
3.5103 \\
3.4439 \\
3.4189 \\
3.3865 \\
3.3468\end{array}$ & $\begin{array}{l}29.0730 \\
40.5704 \\
36.3098 \\
37.8081 \\
38.3457 \\
39.3224 \\
41.4154 \\
41.8552 \\
40.0654 \\
36.6919 \\
40.7925 \\
43.3185 \\
44.7987 \\
45.7532 \\
46.4854 \\
47.1376 \\
47.7575 \\
48.3658 \\
48.9636 \\
49.5572 \\
50.1335 \\
50.6898 \\
51.2257 \\
51.7263 \\
52.6517 \\
53.3962 \\
53.9397 \\
54.2826 \\
54.4754 \\
54.8552 \\
57.5053 \\
59.9301 \\
59.8486 \\
57.8039 \\
54.0897\end{array}$ \\
\hline
\end{tabular}


Figures $3-1$ to 3-8 shows the beam profile at various positions (distance $z$ from the cathode), with the Inline-Injection system of Solenoid + Gun + Solenoid combination inline with the linac. With Solenoid current of I=2140 $\mathbf{~ a m p . ~}$

- BNL RF GUN

60.0
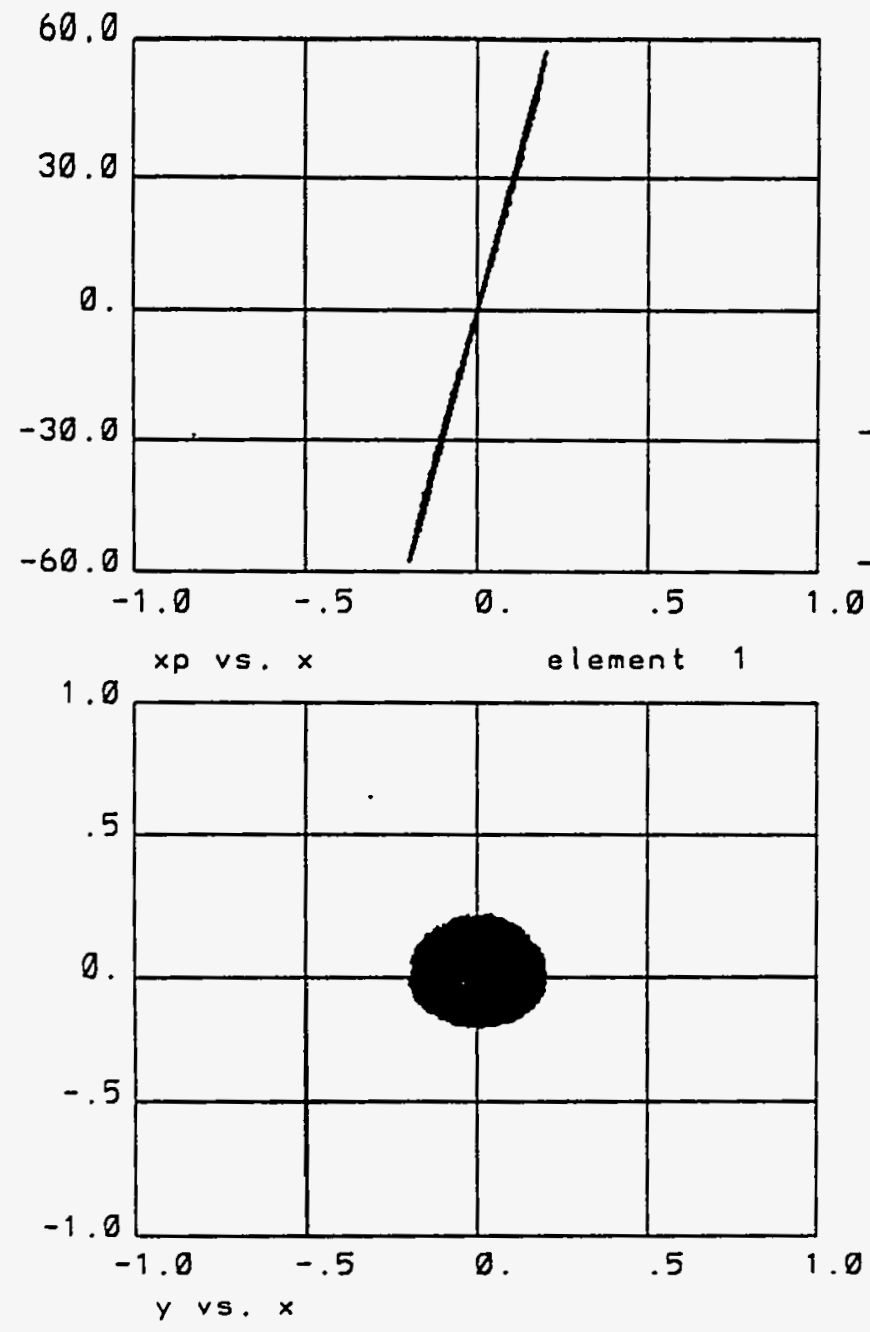

$5,15 p s$ G $(10), 1 \cap C, p h i=43.)^{\circ}$

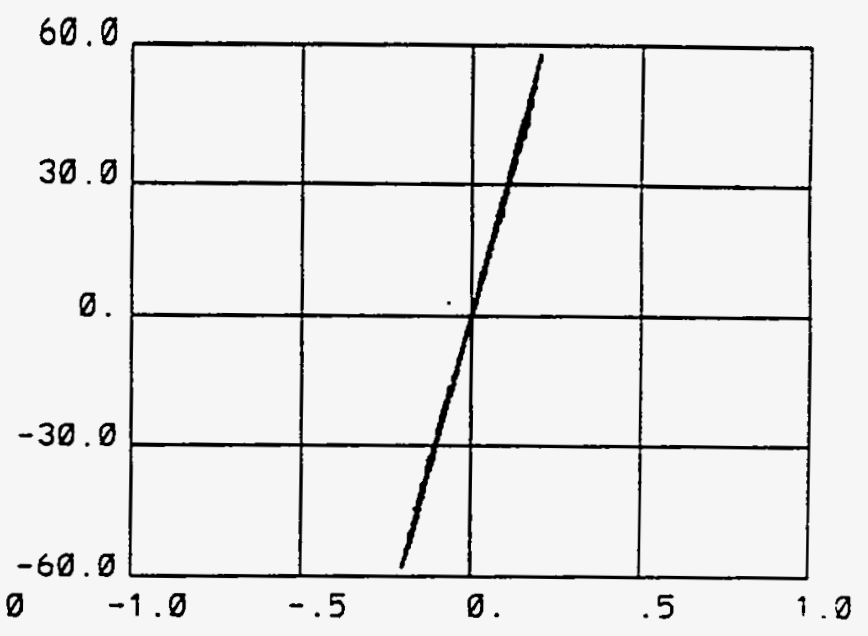

yp vs. y ngood $=6000$
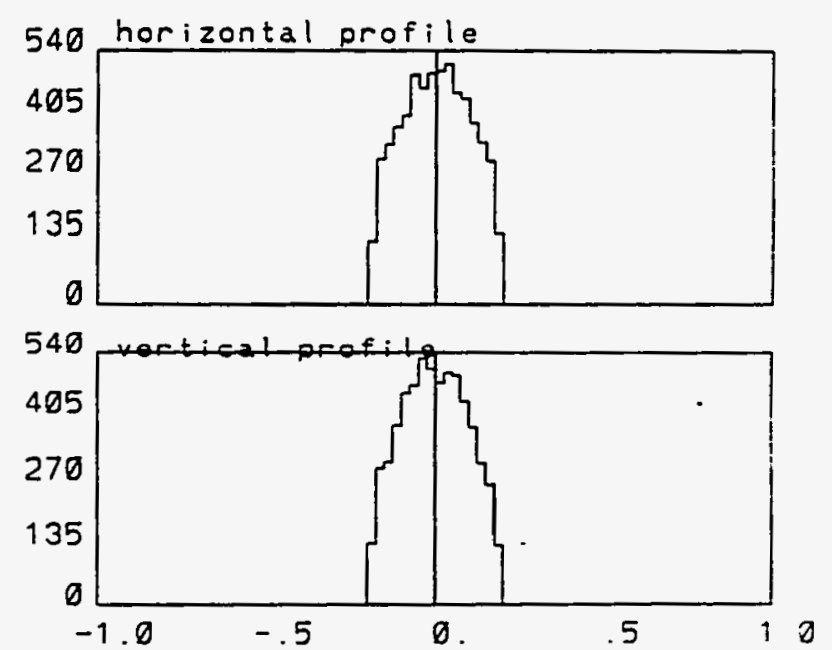

Figure $3-1$ shows the beam profile at position $(n e=1) \quad z=0$, with the Inline-Injection system of Solenoid + Gun + Solenoid combination inline with the linac. With Solenoid current of $I=2140$ amp. 
Figure $3-2$ shows the beam profile at position $(n e=2) z=2.6$, with the Inline-Injection system of Solenoid + Gun + Solenoid combination inline with the linac. With Solenoid current of $I=2140$ amp.

- BNL RF GUN
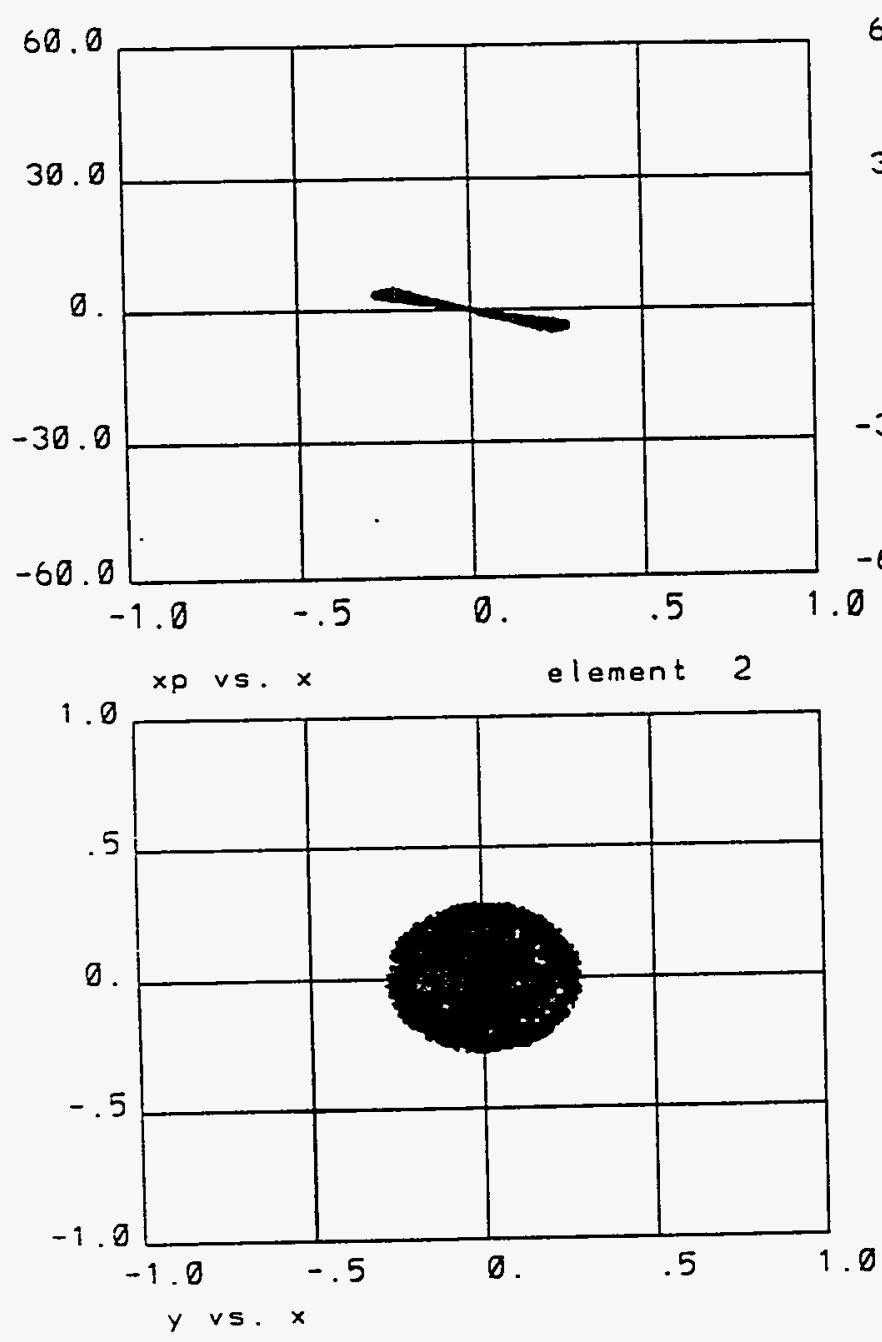

$5.15 p s G(10), 1 n C$, phi $=43.)^{\text {. }}$
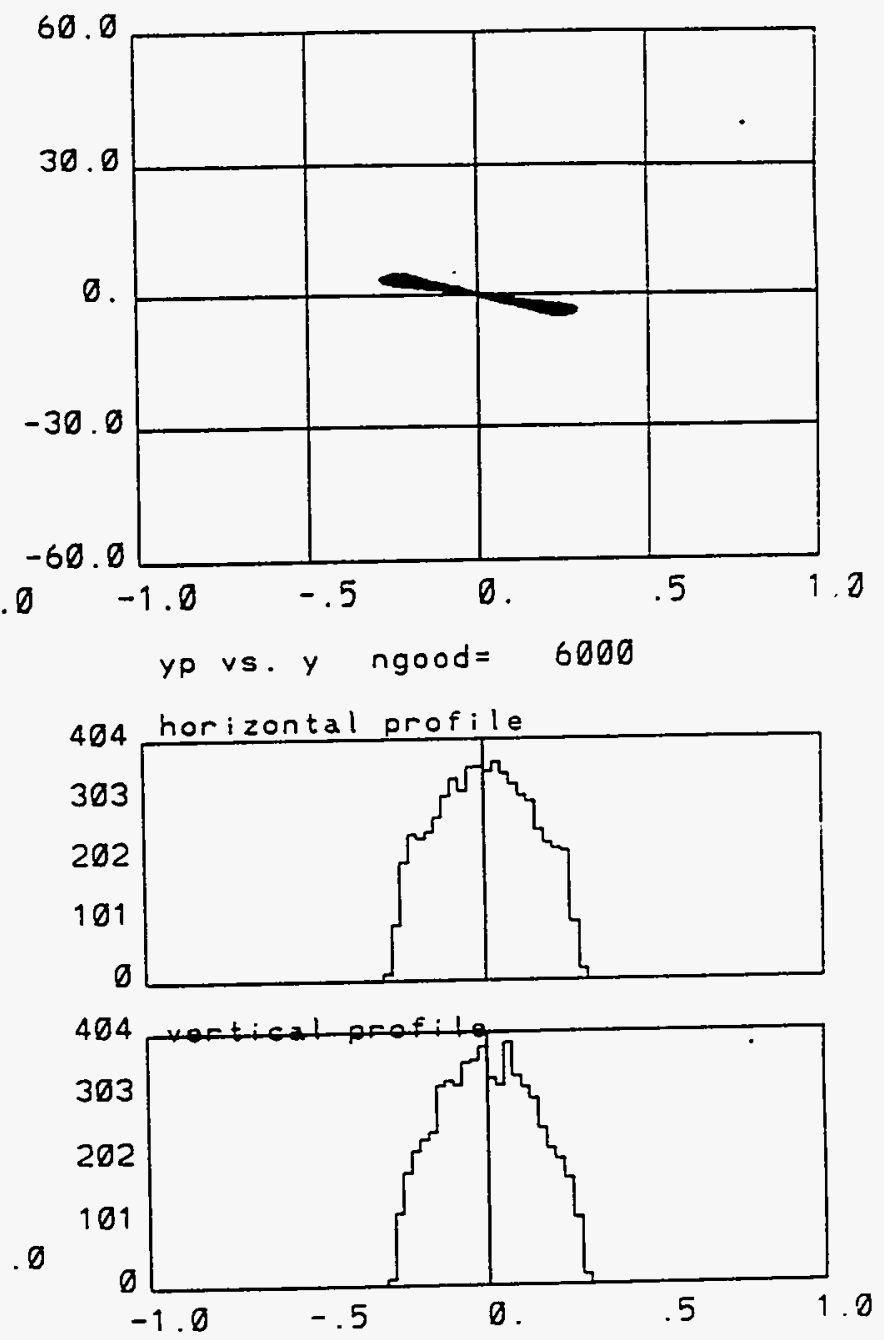
Figure $3-3$ shows the beam profile at position $(n e=3) \quad z=5.2$, with the Inline-Injection system of Solenoid + Gun + Solenoid combination inline with the linac. With Solenoid current of $\mathrm{I}=2140$ amp.

- BNL RF GUN
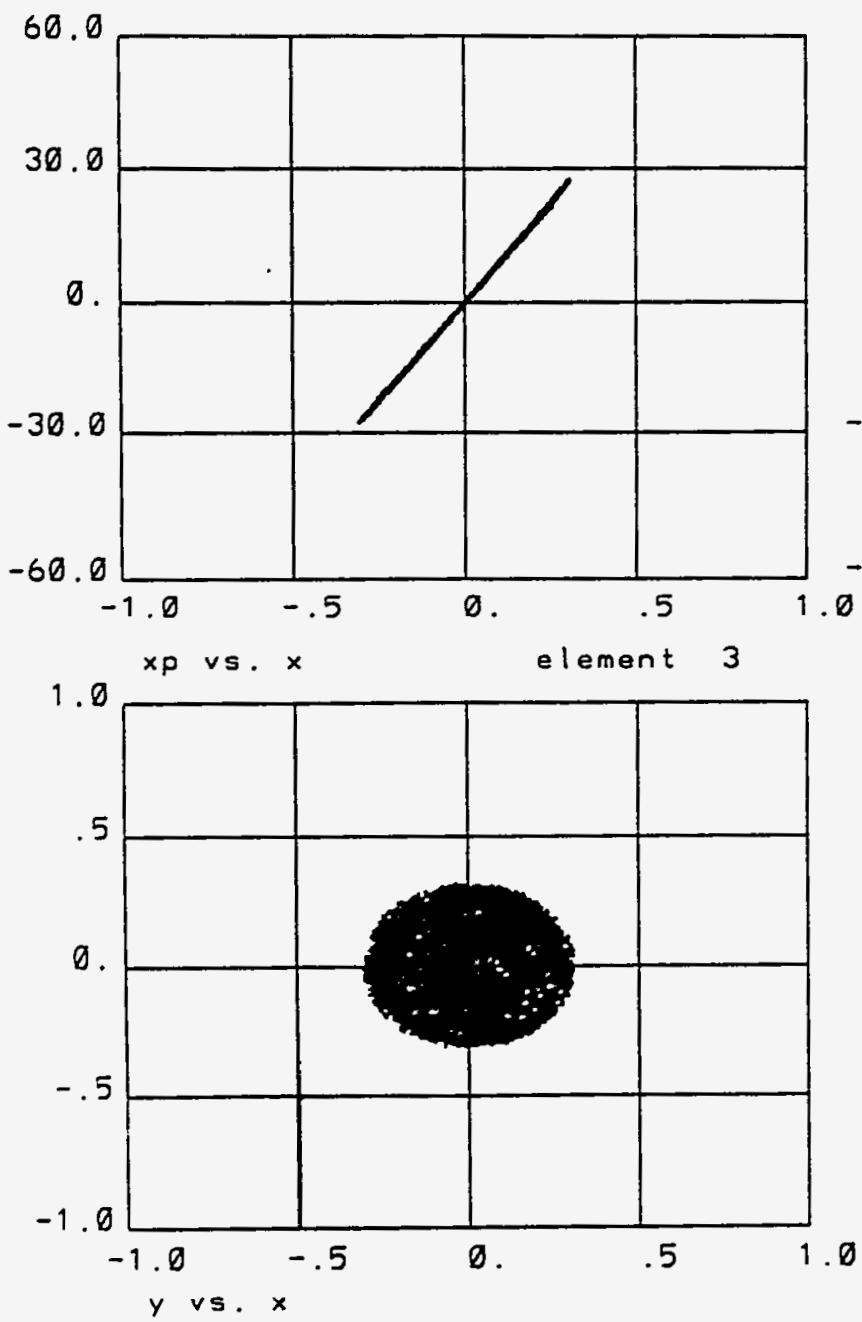

$5.15 p s G(10), 1 n C$, phi $=43.)^{\circ}$

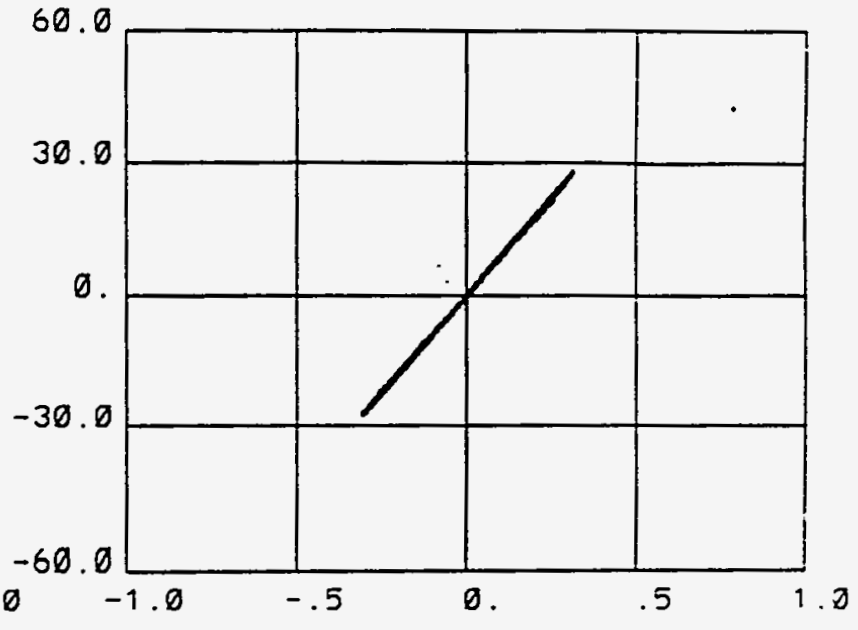

yp vs. Y ngood $=6000$
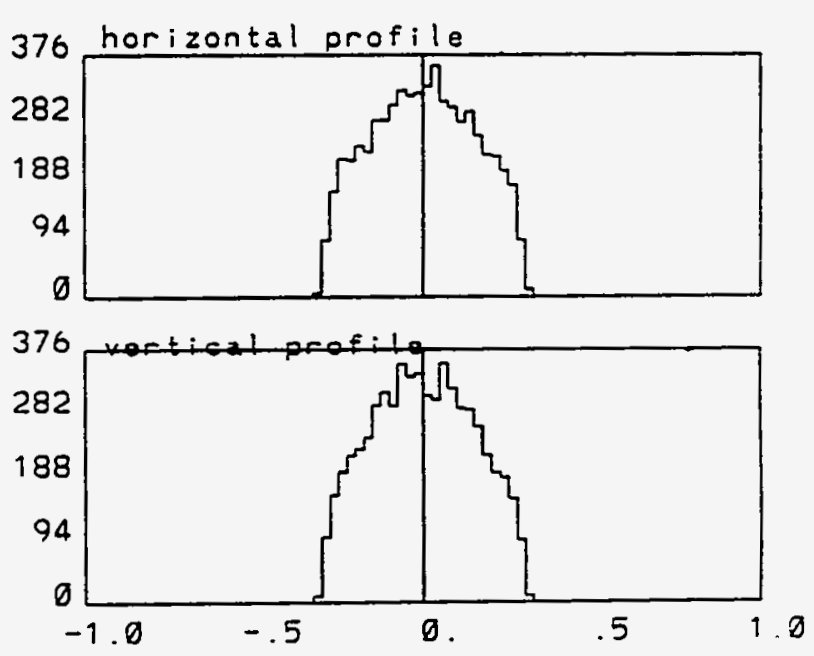
Figure $3-4$ shows the beam profile at position $(n e=4) z=7.9$, with the Inline-Injection system of Solenoid + Gun + Solenoid combination inline with the linac. With Solenoid current of $I=2140 \mathrm{amp}$.

- BNL RF GUN
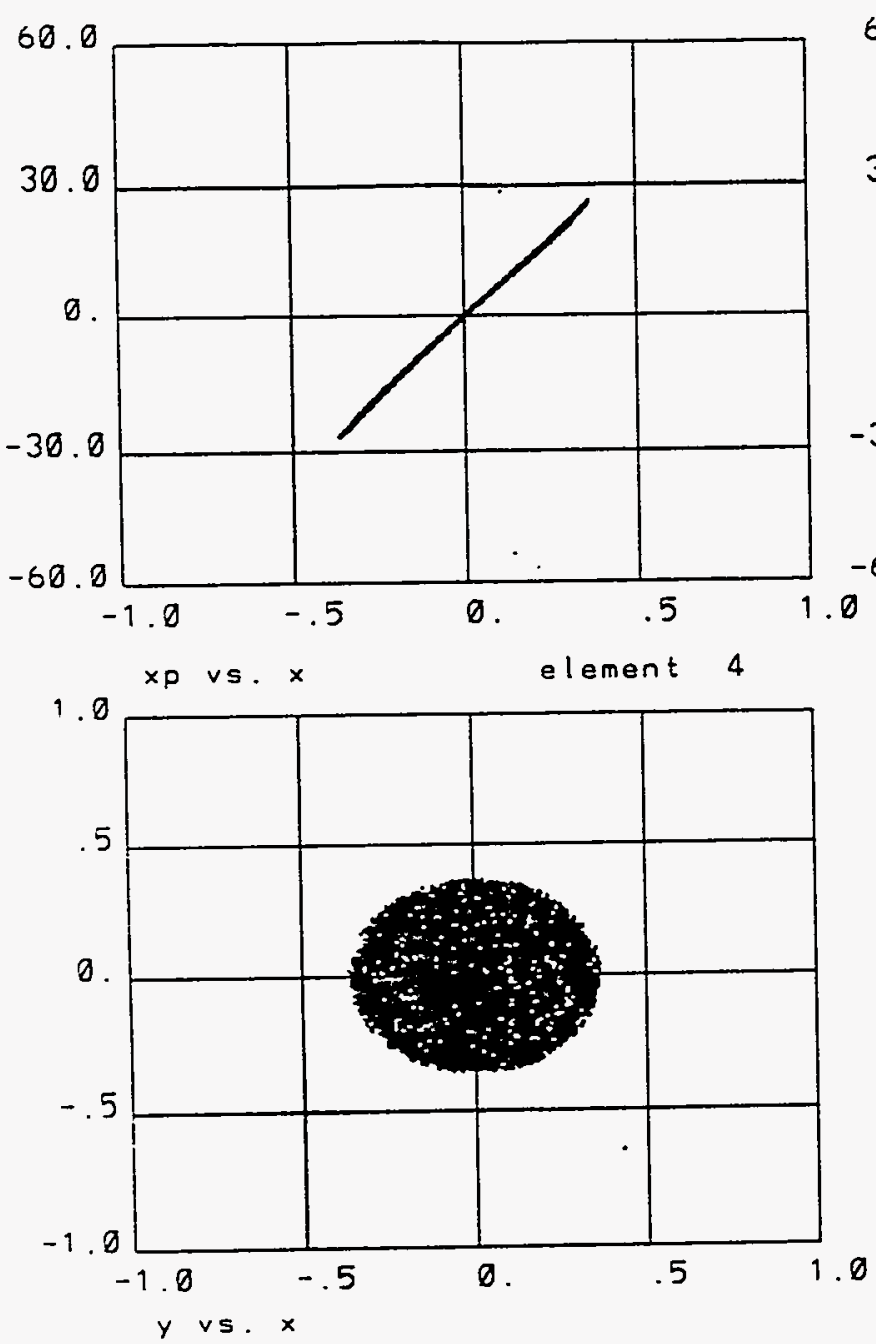

$5.15 p$ s $G(10), 1 \cap C, p h i=43.1$.
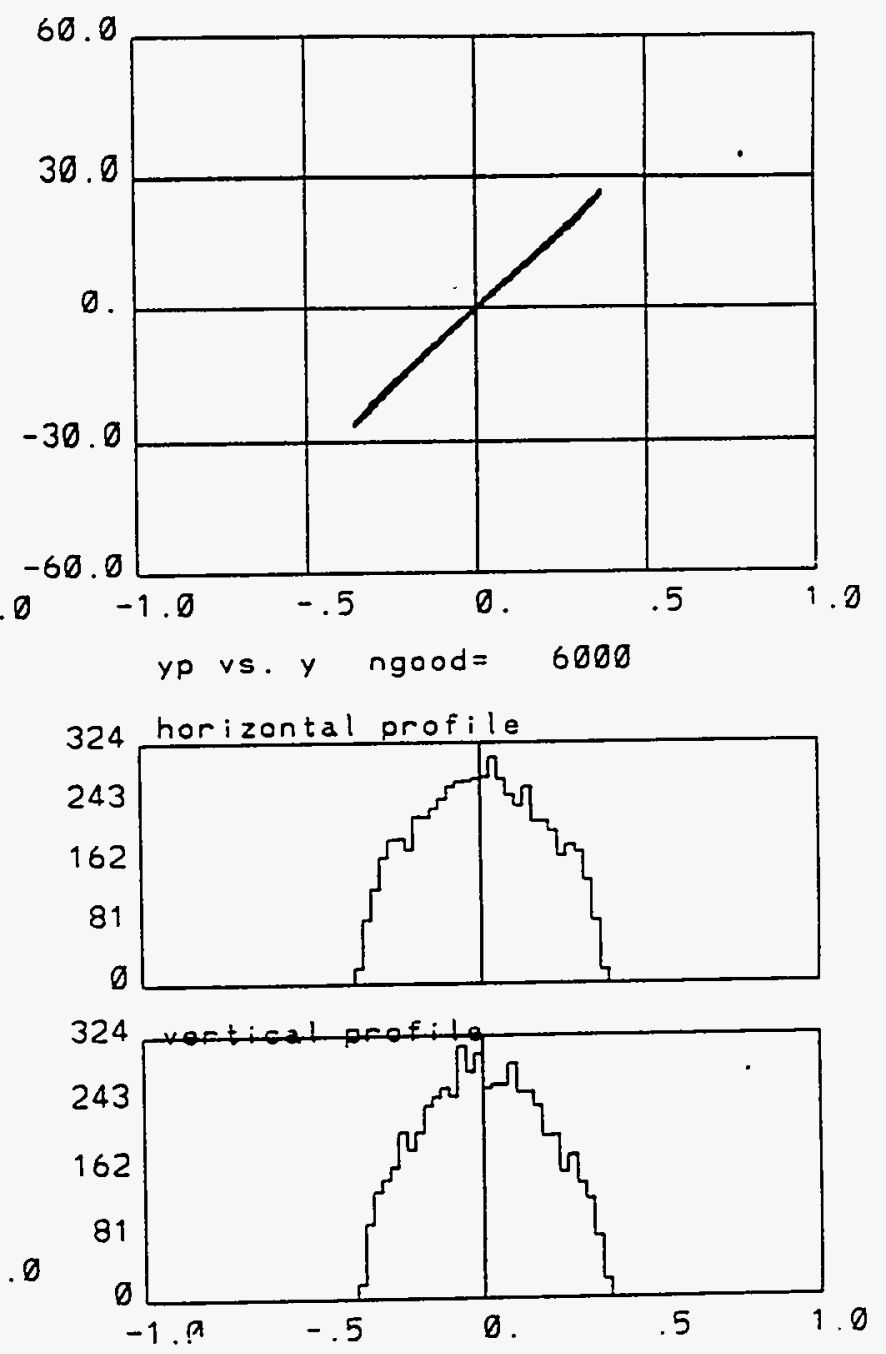
Figure $3-5$ shows the beam profile at position (ne $=5$ ) $z=10$, with the Inline-Injection system of Solenoid + Gun + Solenoid combination inline with the linac. With Solenoid current of $I=2140 \mathrm{amp}$.

- BNL RF GUN

60.0

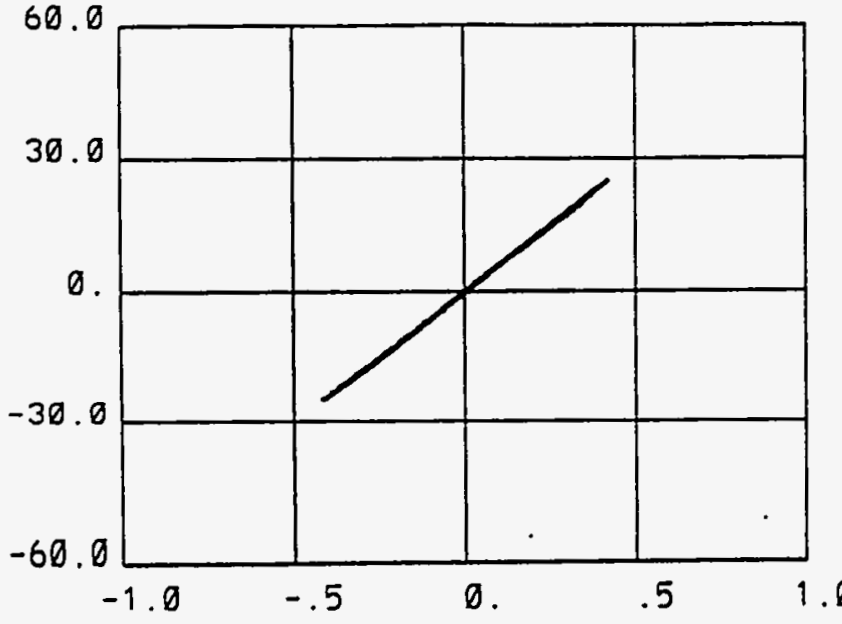

$x p$ v . $x$ element 5

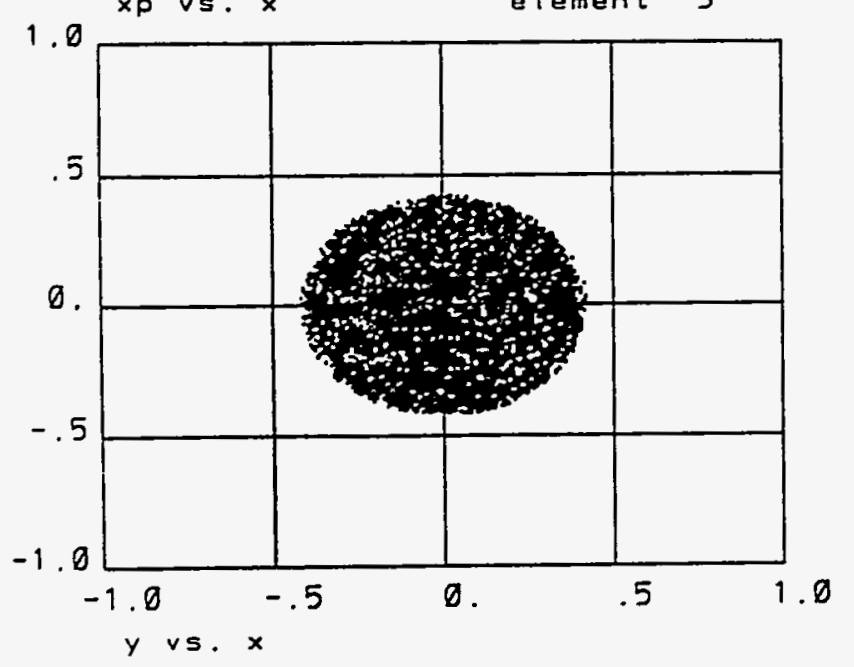

5.15ps $G(10), 1 \mathrm{nC}$, phi $=43.1$.
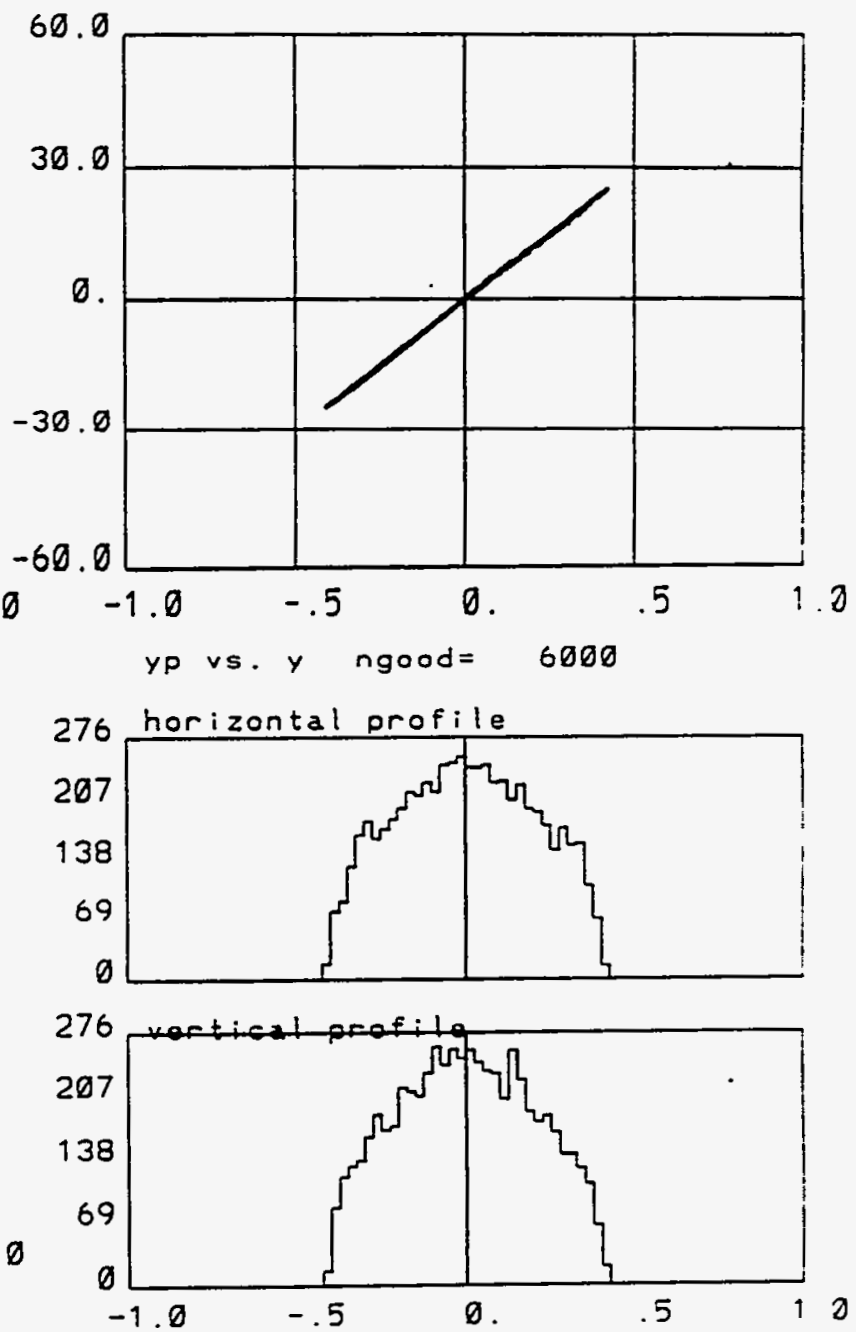
Figure 3 -6 shows the beam profile at position $(n e=10) \quad z=20$, with the Inline-Injection system of Solenoid + Gun + Solenoid combination inline with the linac. With Solenoid current of $I=2140 \mathrm{amp}$.

- BNL RF GUN $(R=.09 \text { gaus }(1 \mathrm{~mm}) .5 .15 p s \text { G }(10), 1 n C \text {, phi }=43 .)^{\circ}$
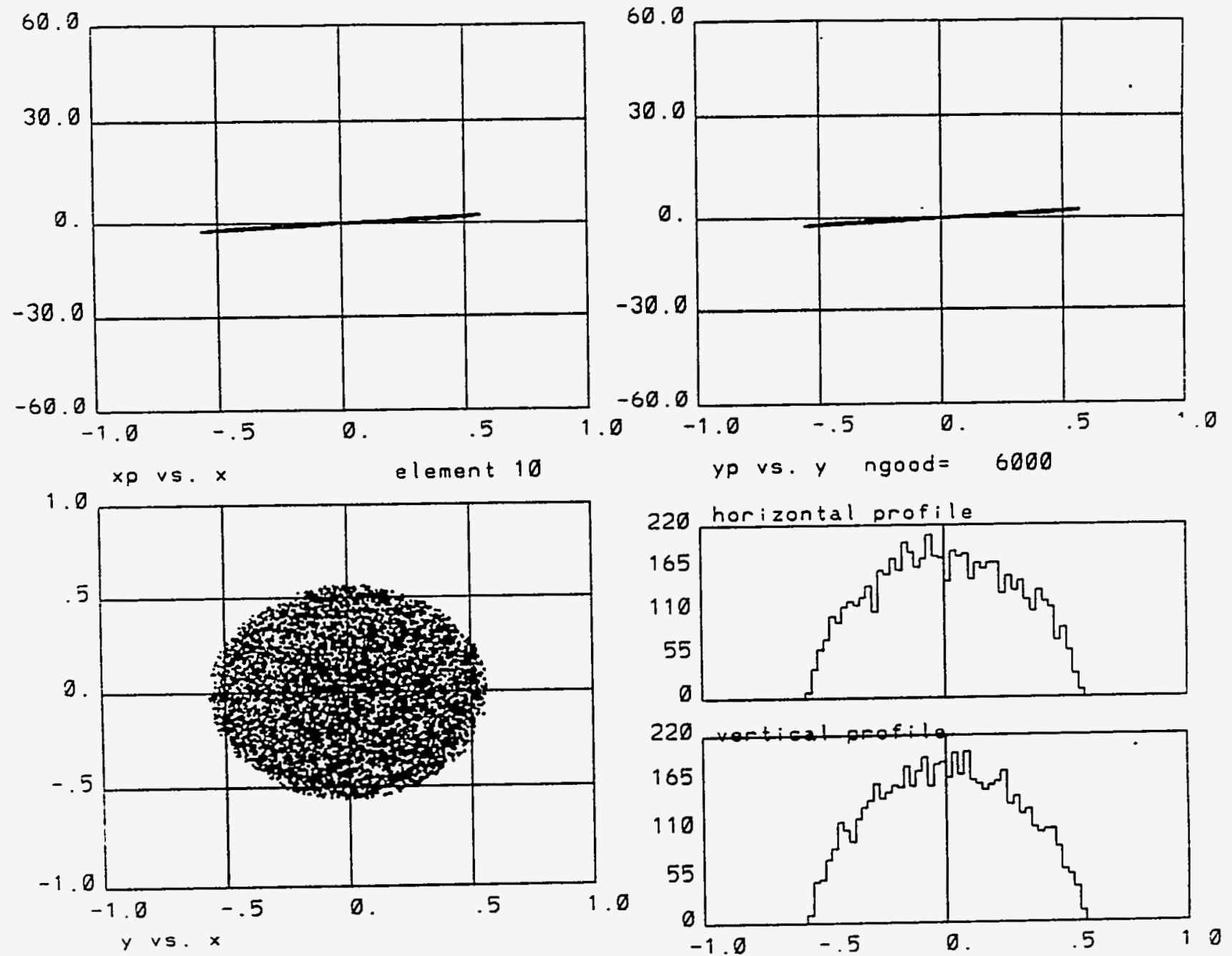
Figure $3-7$ shows the beam profile at position $(n e=20) z=40$, with the Inline-Injection system of Solenoid + Gun + Solenoid combination inline with the linac. With Solenoid current of $I=2140$ amp.

- BNL RF GUN

60.
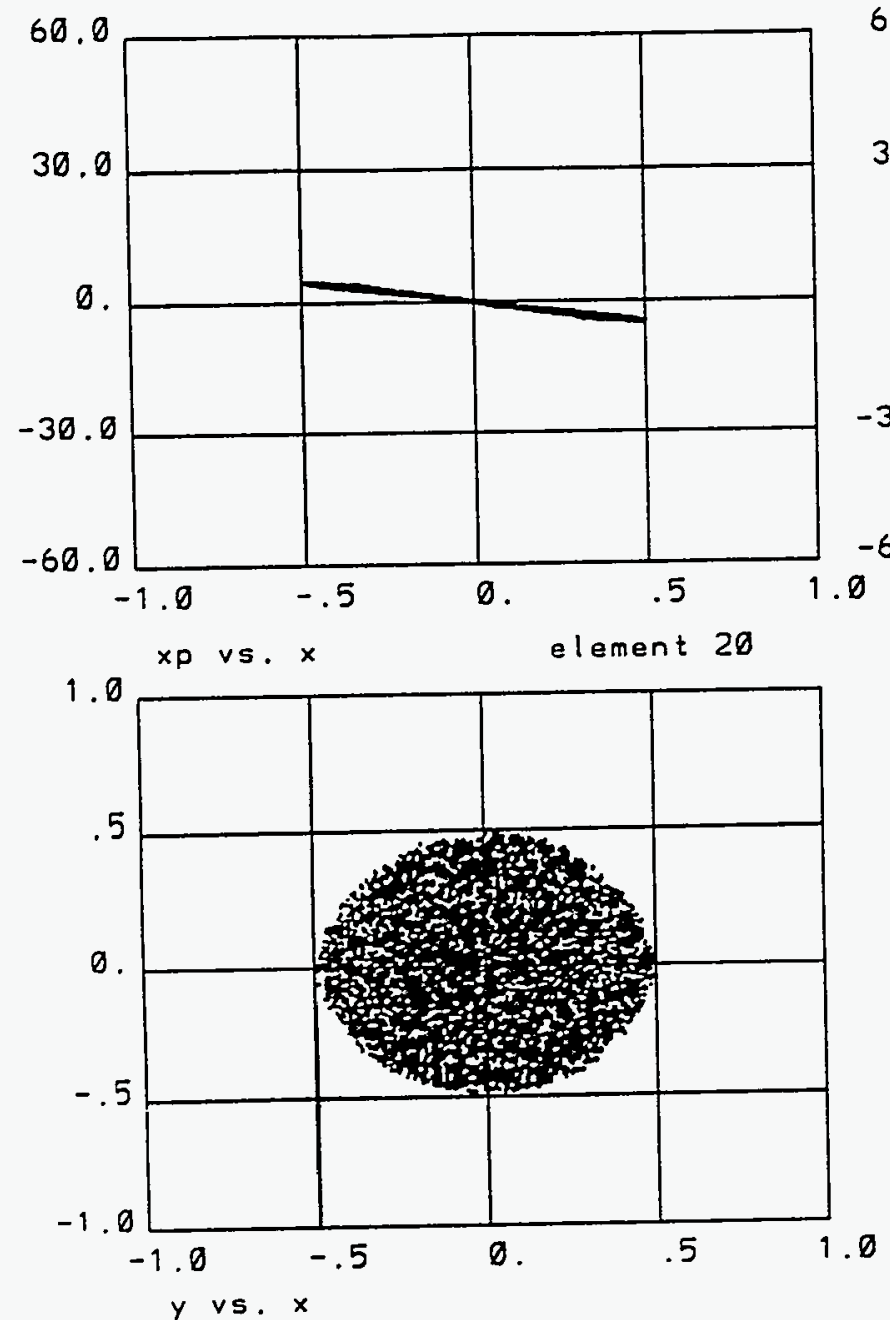

$5.15 p s G(10), 1 n C$, ohi $=43.)^{\circ}$
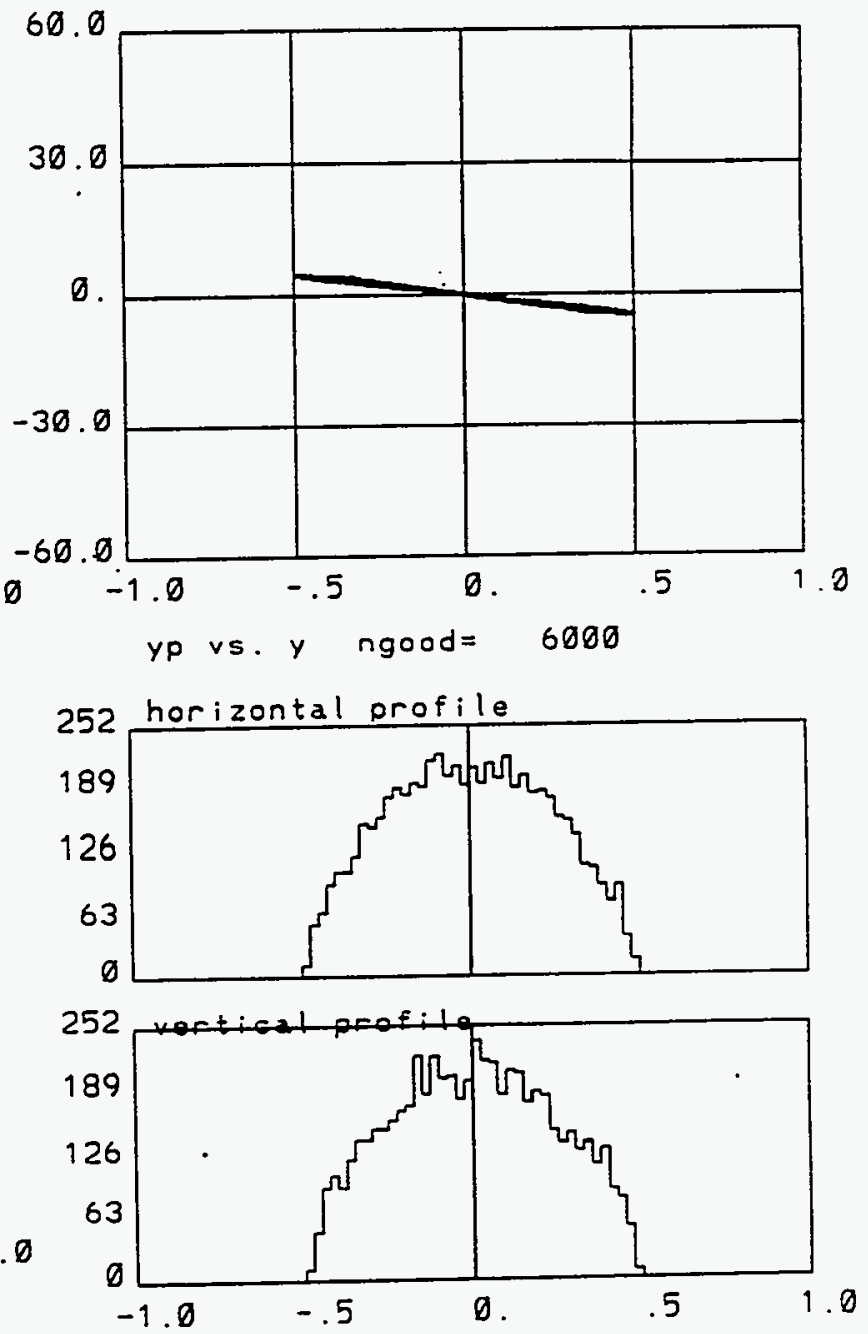
Figure $3-8$ shows the beam profile at position $(n e=30) z=70$, with the Inline-Injection system of Solenoid + Gun + Solenoid combination inline with the linac. With Solenoid current of $I=2140 \mathrm{amp}$.

-BNL RF GUN

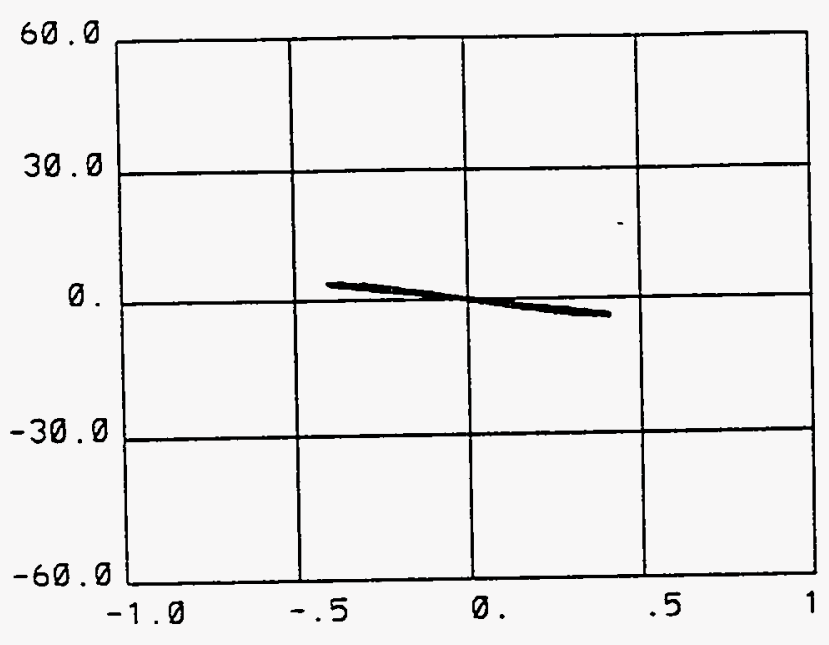

$x$ vs. $x$ element 30

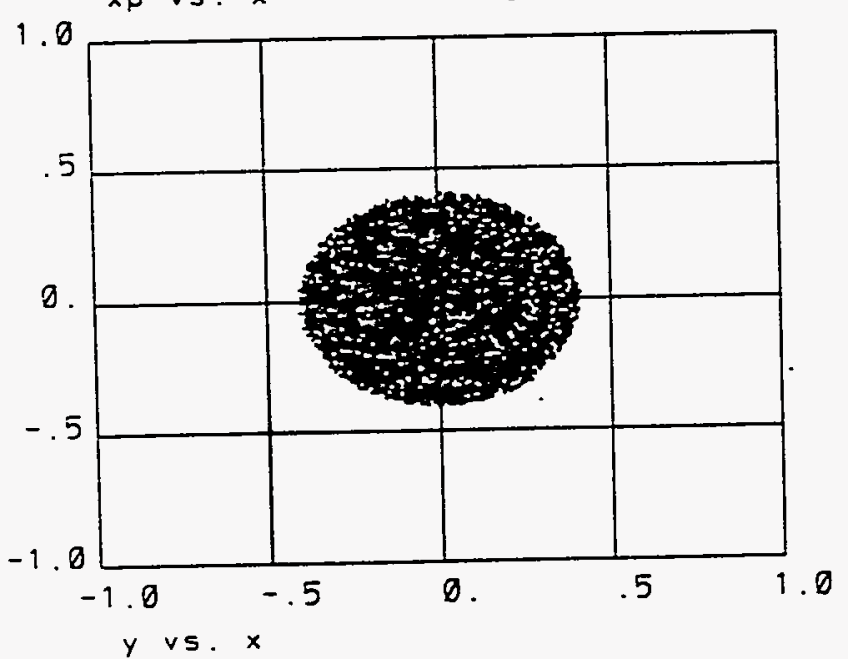

$5.15 p s G(10), 1$ nC, phi $=43.1^{\text {. }}$
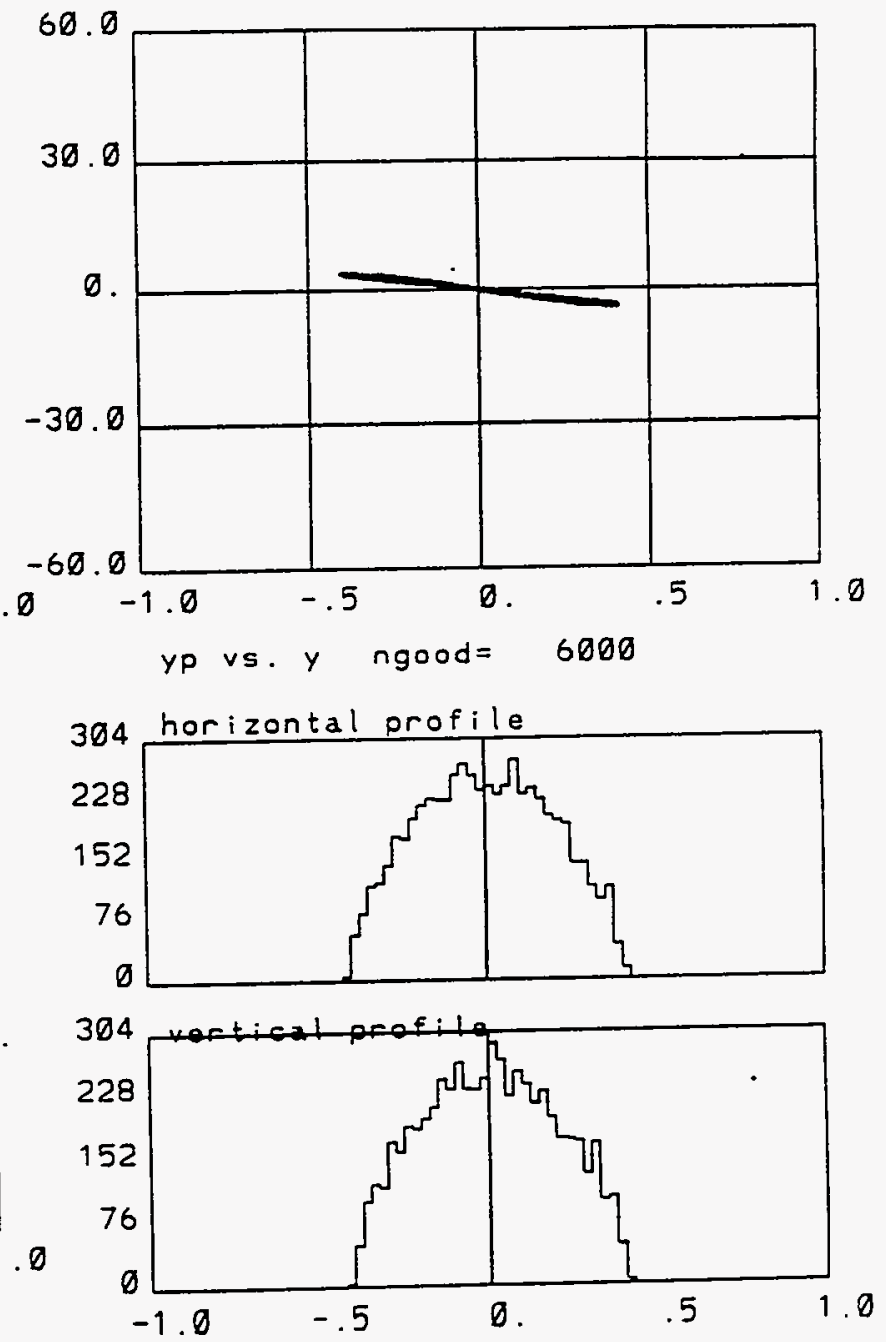
Figures 4-1 to 4-8 shows the beam profile at various positions (distance 2 from the cathode), with the Inline-Injection system of Solenoid + Gun + Solenoid combination inline with the linac. With Solenoid current of I =2140 amp.

'BNL RF GUN
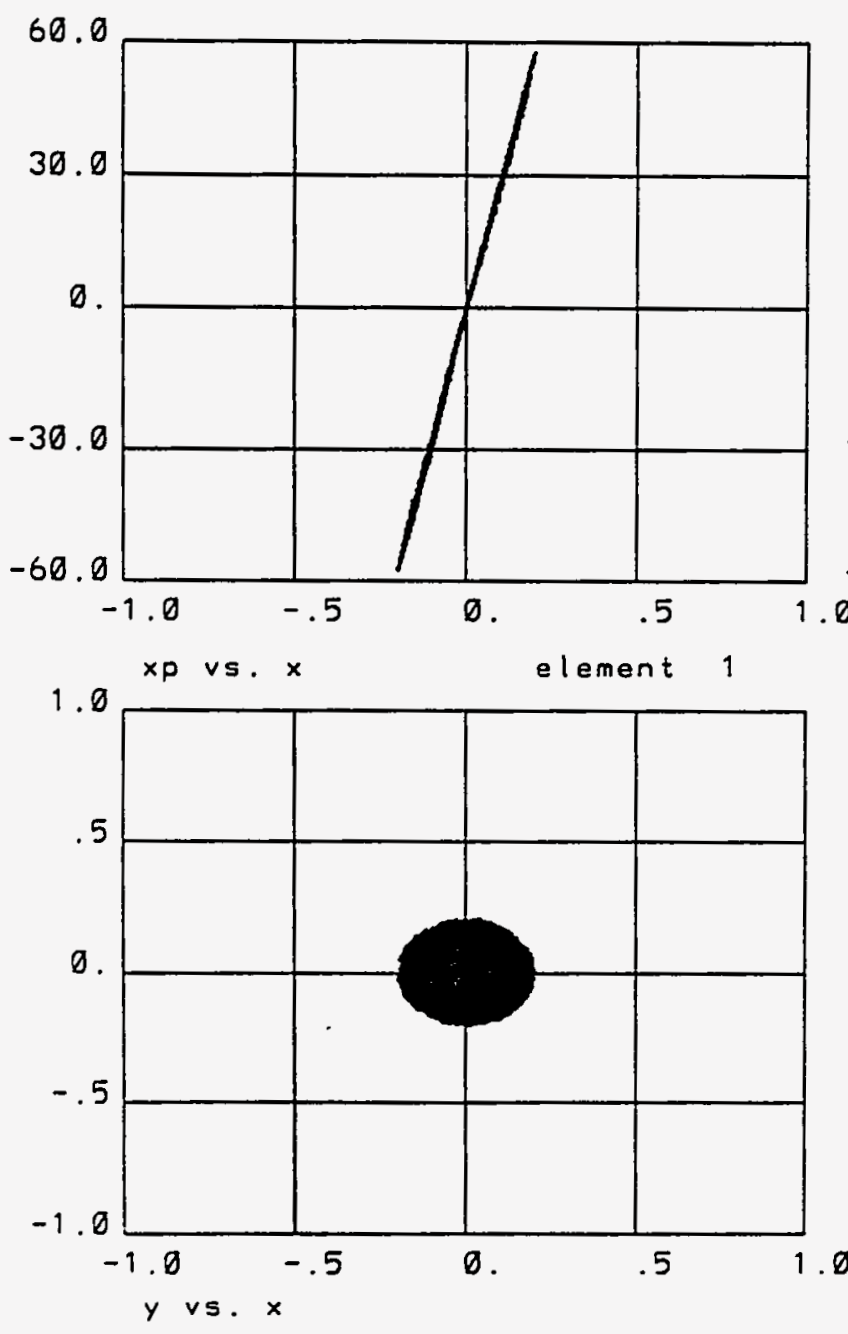

$5.15 p s G(10), 1 n C$, ph $i=43.1$

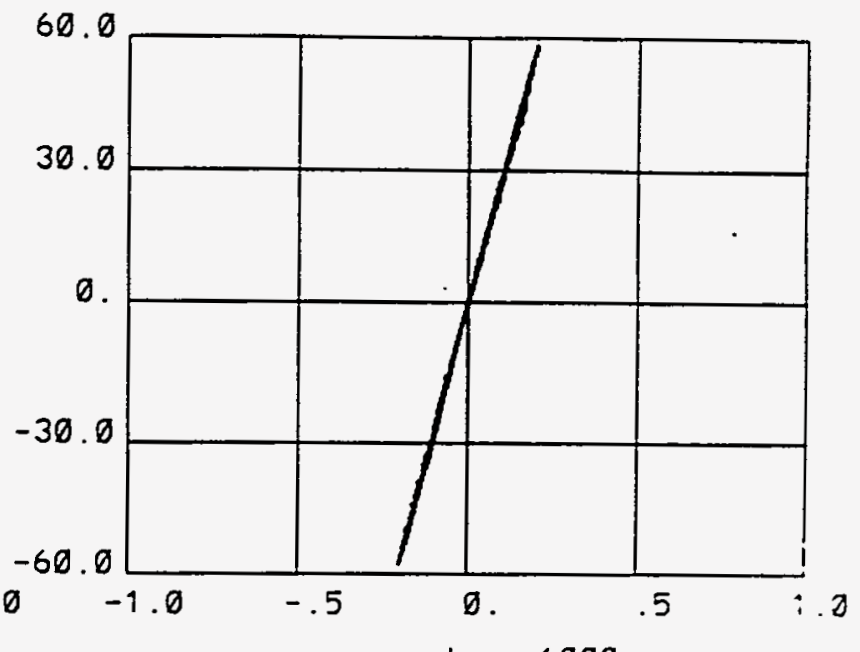

yp vs. y ngood $=6000$
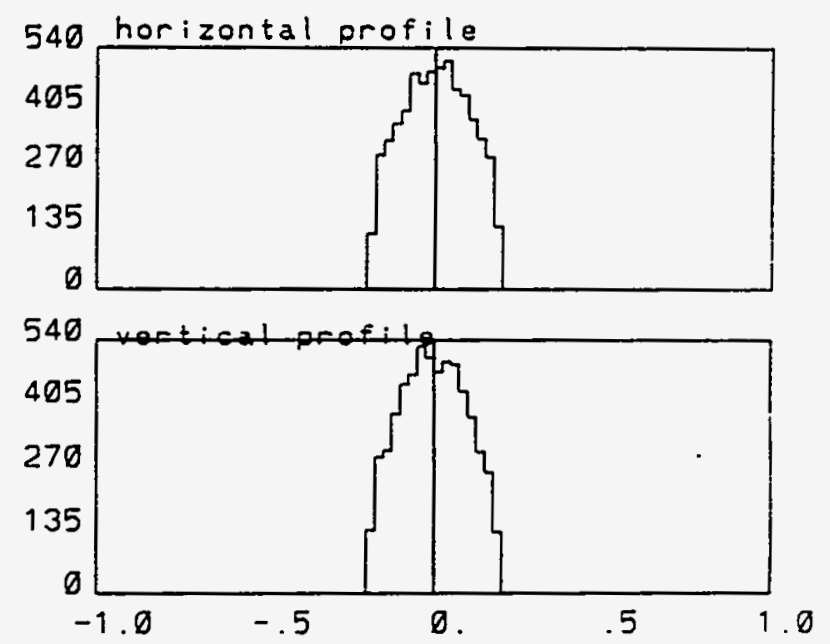

Figure $4-1$ shows the beam profile at position $(n e=1) \tau=0$, with the Inline-Injection system of Solenoid + Gun + Solenoid combination inline with the linac. With Solenoid current of $\mathrm{I}=2160 \mathrm{amp}$. 
Figure $4-2$ shows the beam profile at position $(n e=2) z=2.6$, with the Inline-Injection system of Solenoid + Gun + Solenoid combination inline with the linac. With Solenoid current of $I=2160$ amp.

-BNL RF GUN
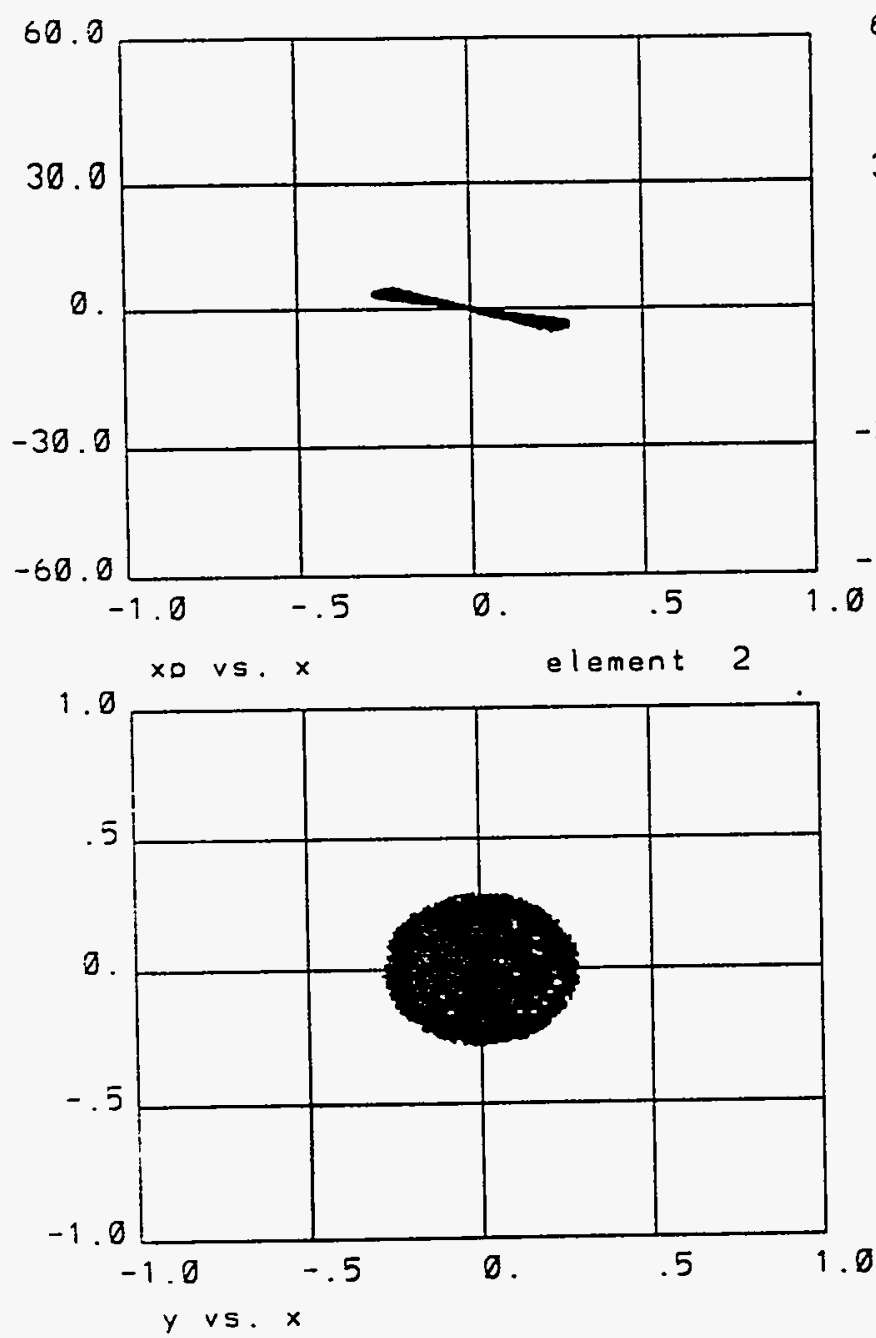

$5.15 p s$ G $(10), 1$ nC, phi $=43.1^{\circ}$
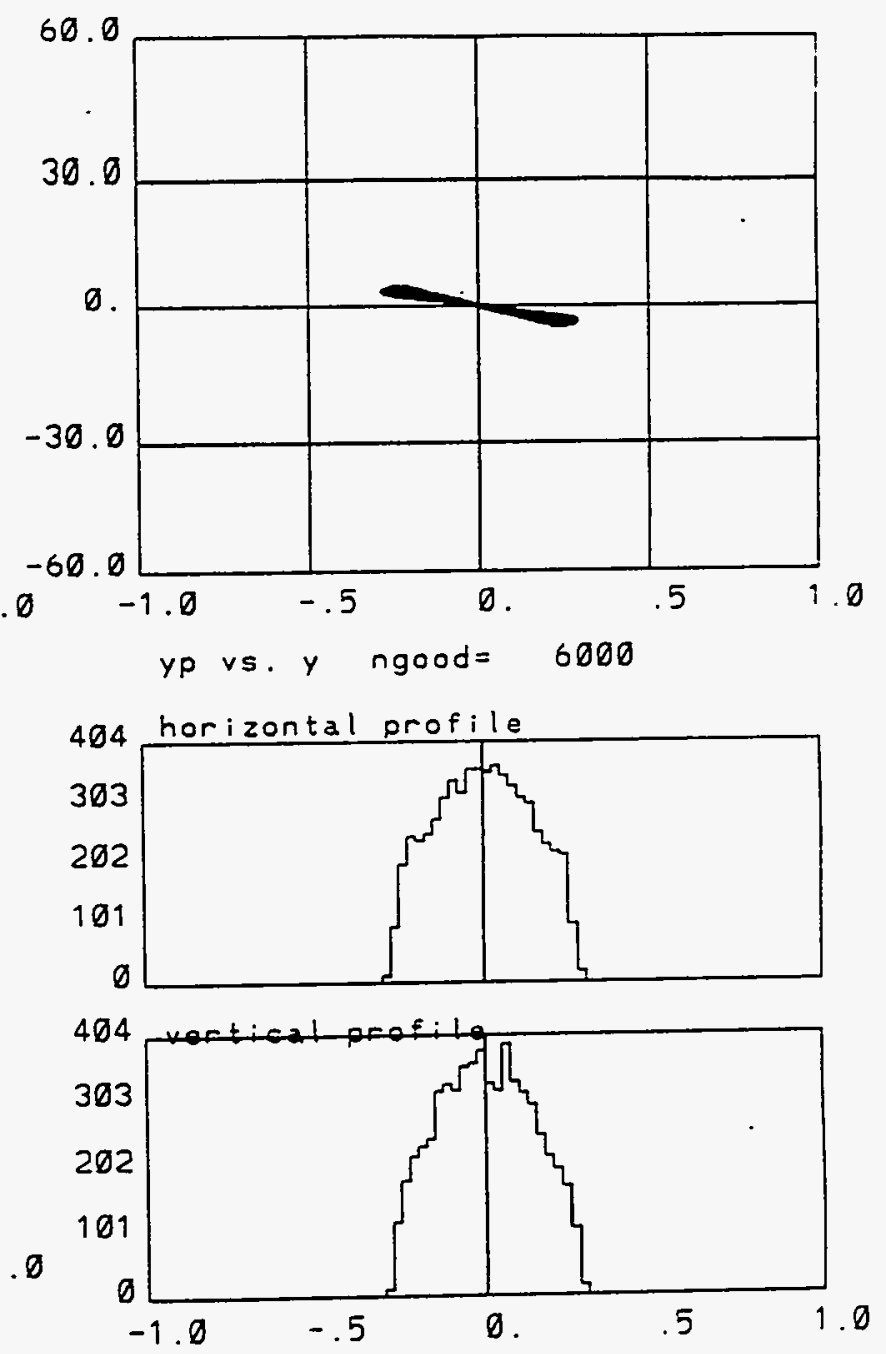
Figure $4-3$ shows the beam profile at position $(n e=3) z=5.2$, with the Inline-Injection system of Solenoid + Gun + Solenoid combination inline with the linac. With Solenoid current of $\mathrm{I}=2160 \mathrm{amp}$.

- BNL RF GUN
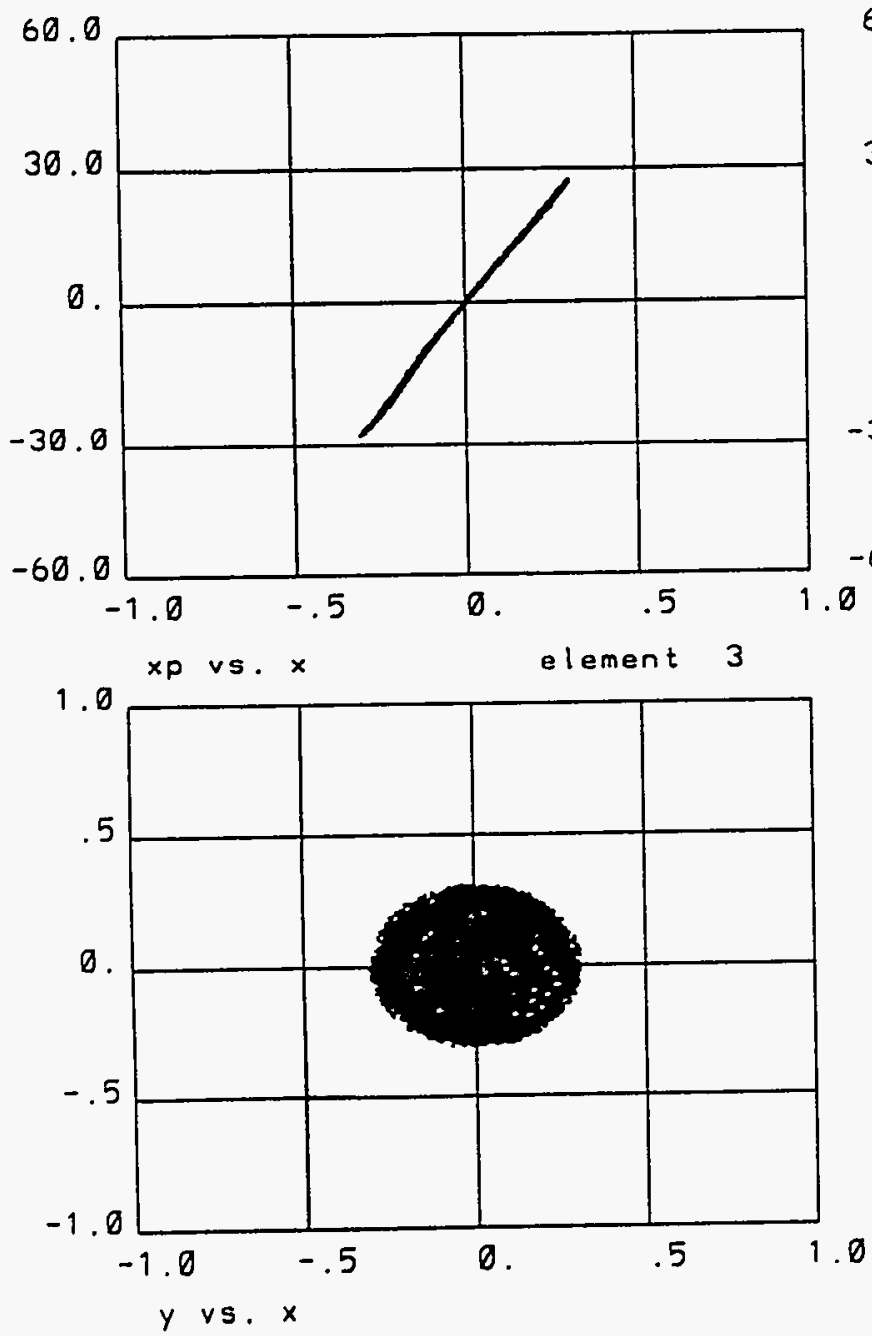

$5.15 p s$ s $(10), 1$ nC, phi=43.1.
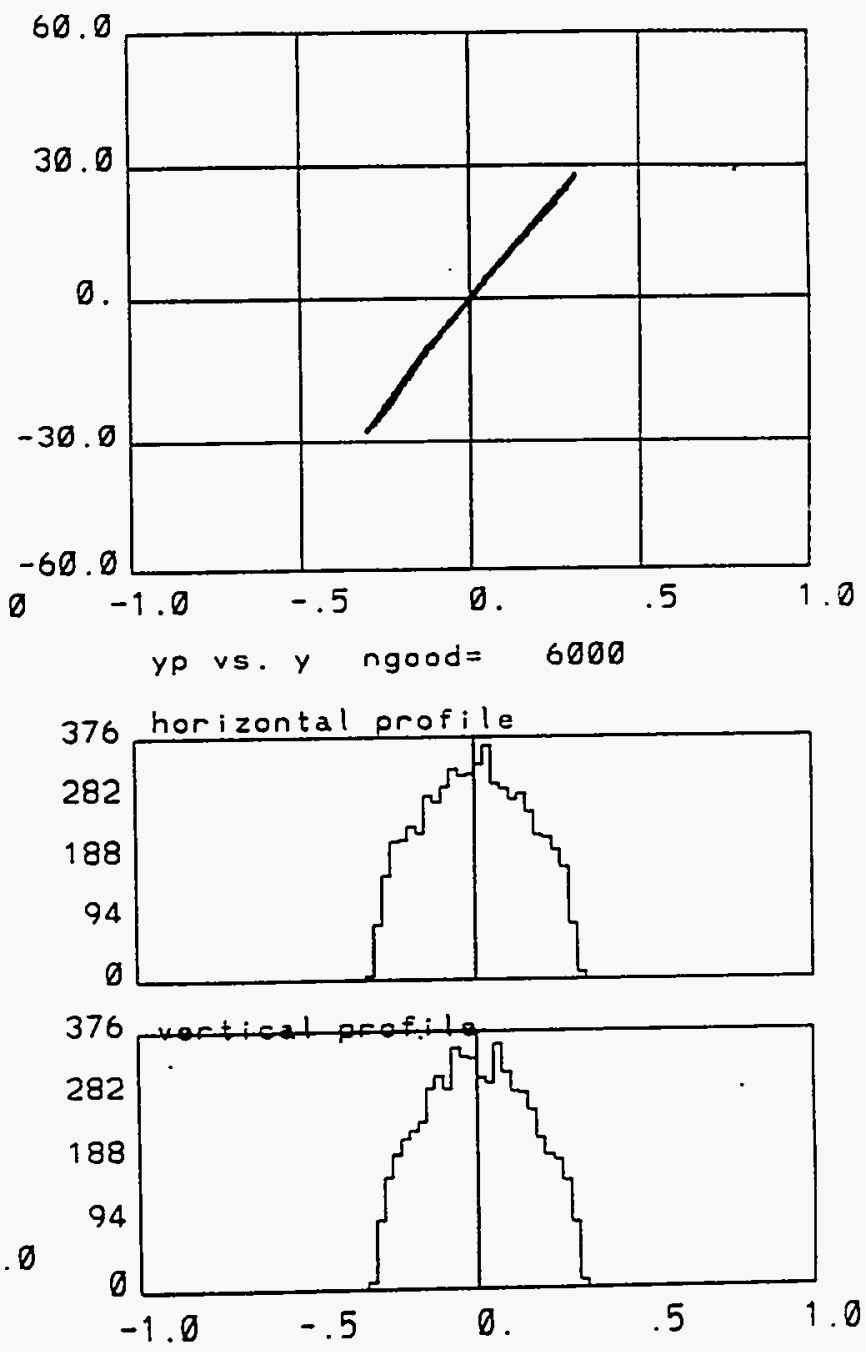
Figure $4-4$ shows the beam profile at position $(n e=4) z=7.9$, with the Inline-Injection system of Solenoid + Gun + Solenoid combination inline with the linac. With Solenoid current of $I=2160 \mathrm{amp}$.

- BNL RF GUN
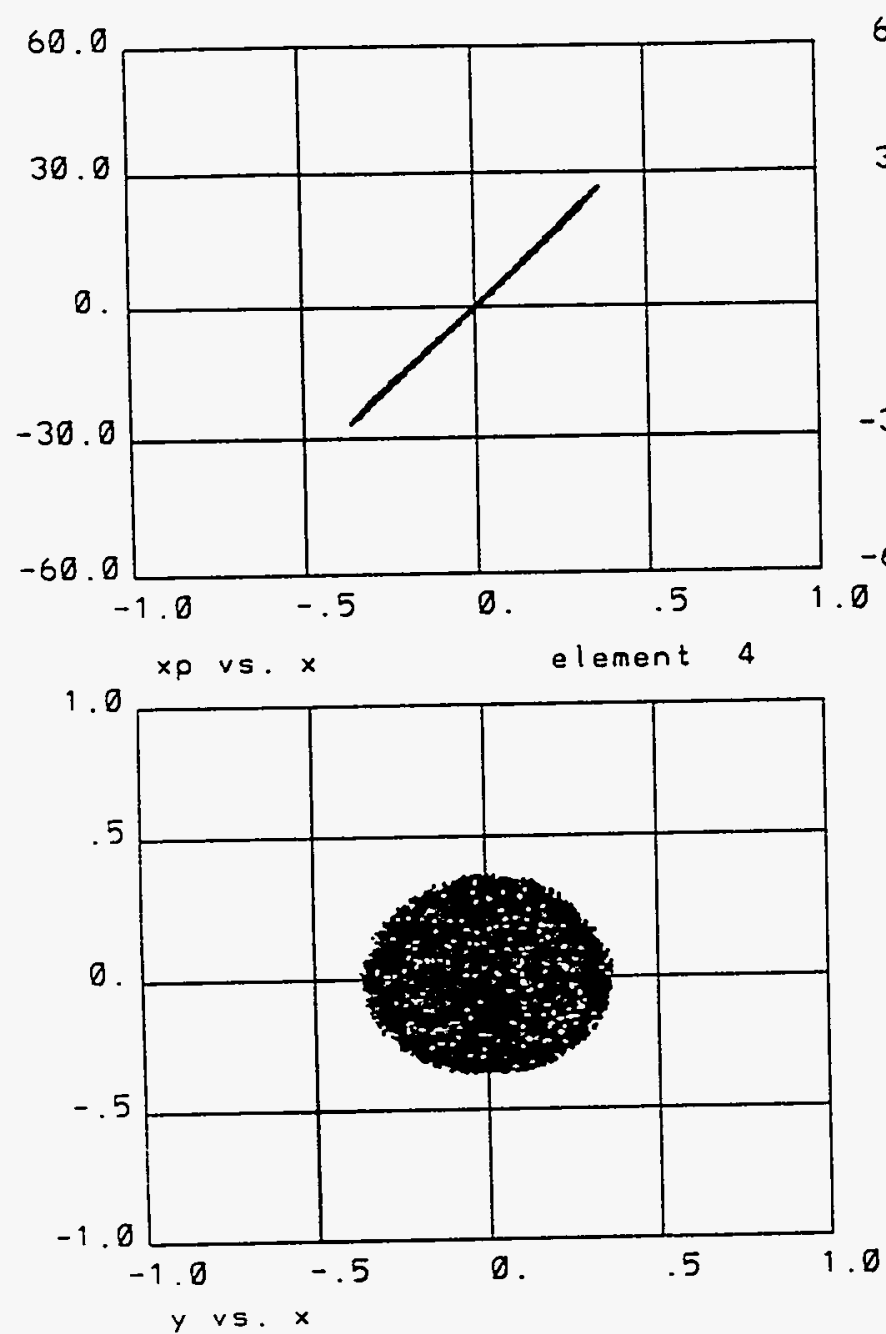

$5.15 p s G(10), 1 \cap C$, phi $=43.)^{\circ}$
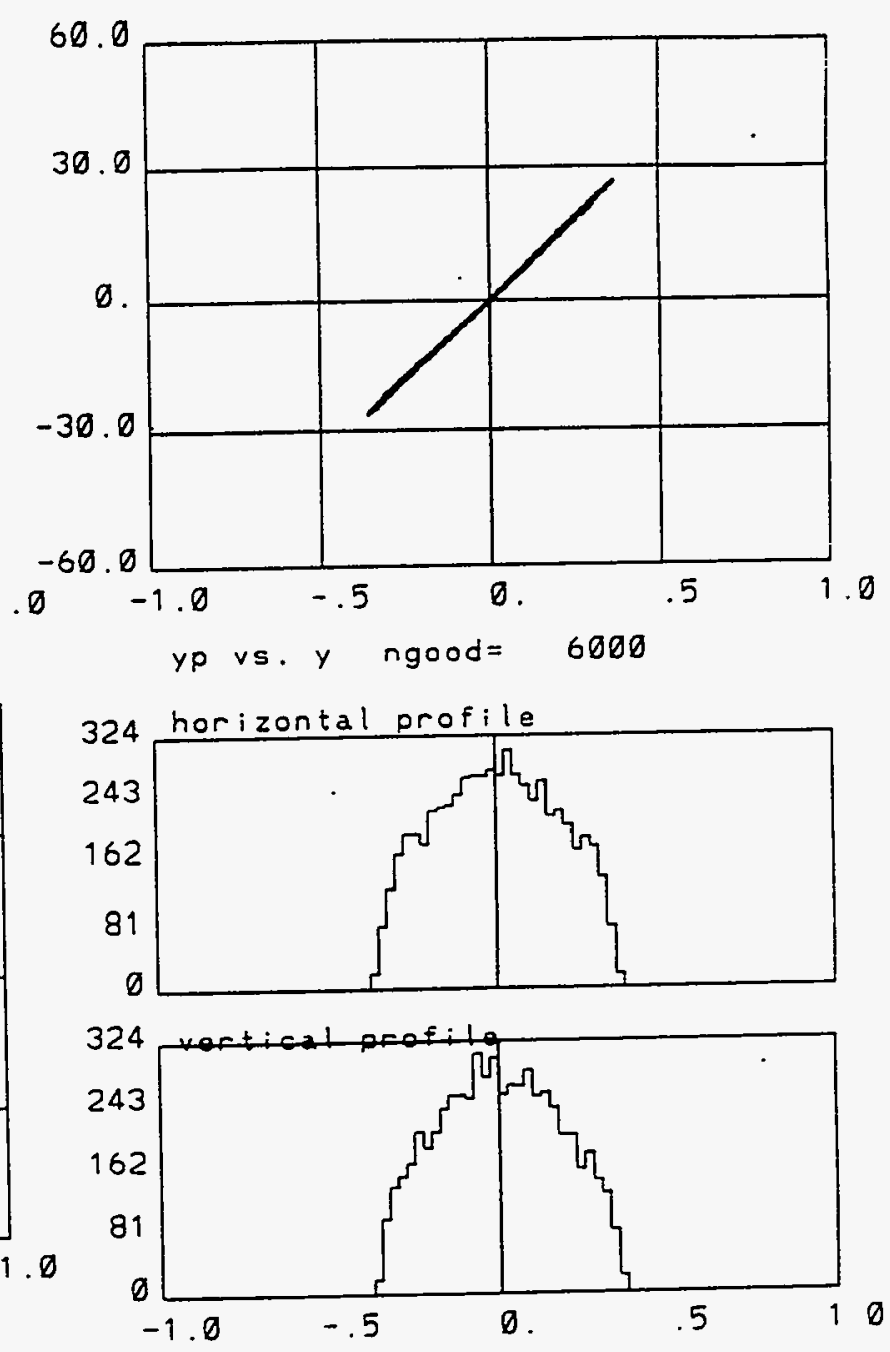
Figure $4-5$ shows the beam profile at position $(\mathrm{ne}=5) z=10$, with the Inline-Injection system of Solenoid + Gun + Solenoid combination inline with the linac. With Solenoid current of $I=2160$ amp.

- BNL RF GUN
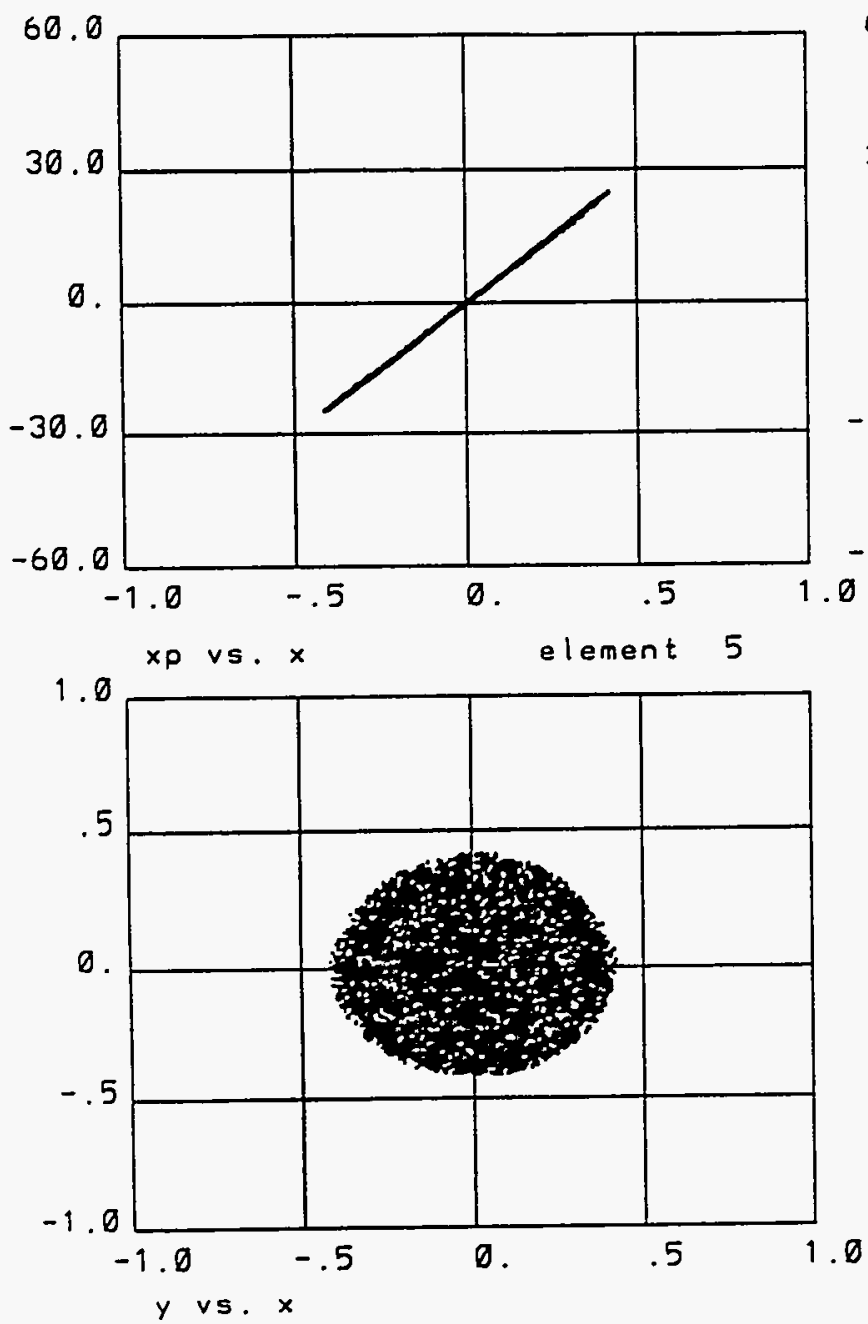

5.15ps $G(10), 1 n C$, phi $=43.)^{\circ}$

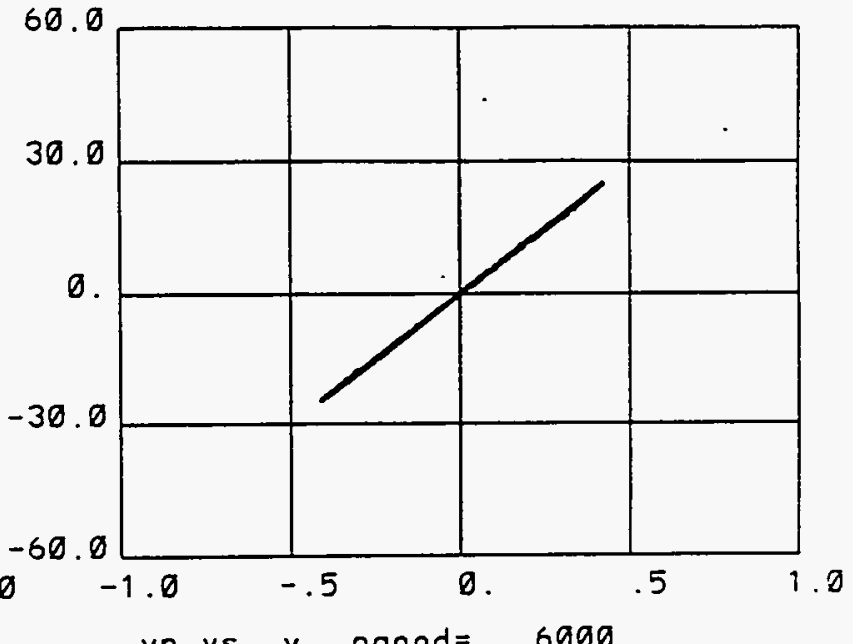

yp vs. y ngood $=6000$
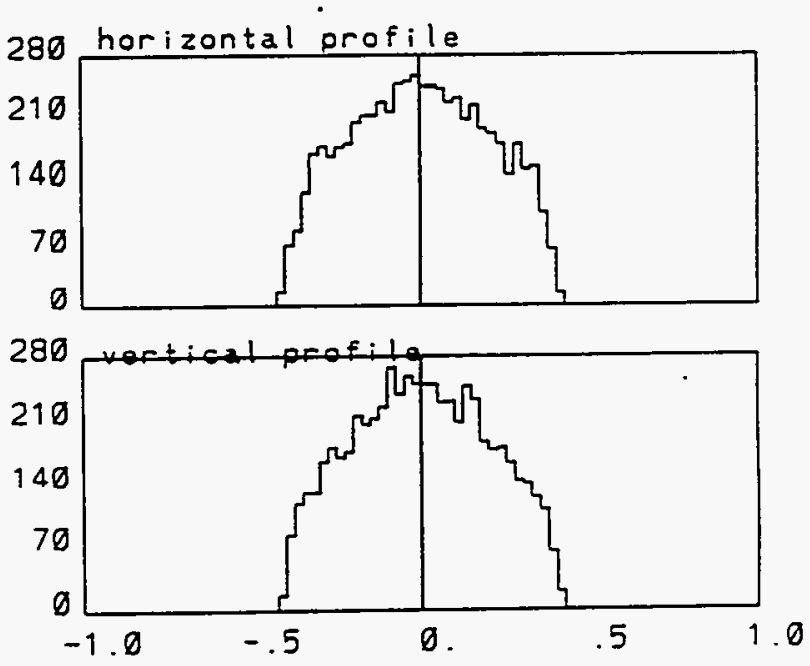
Figure 4 -6 shows the beam profile at position (ne=10) $z=20$, with the Inline-Injection system of Solenoid + Gun + Solenoid combination inline with the linac. With Solenoid current of $I=2160 \mathrm{amp}$.

- BNL RF GUN
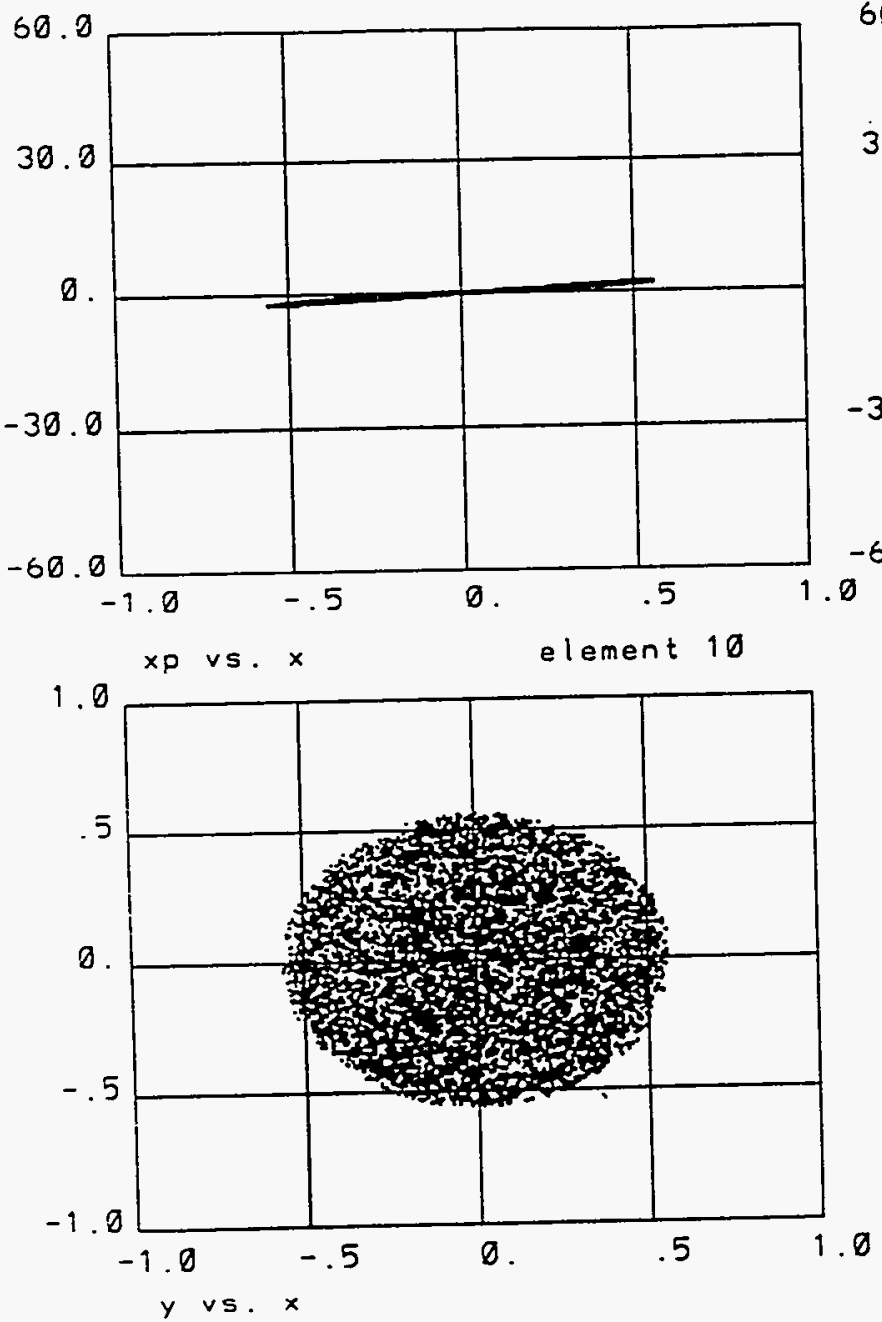

$5.15 p s G(10), 1$ nC, phi $=43.1$.
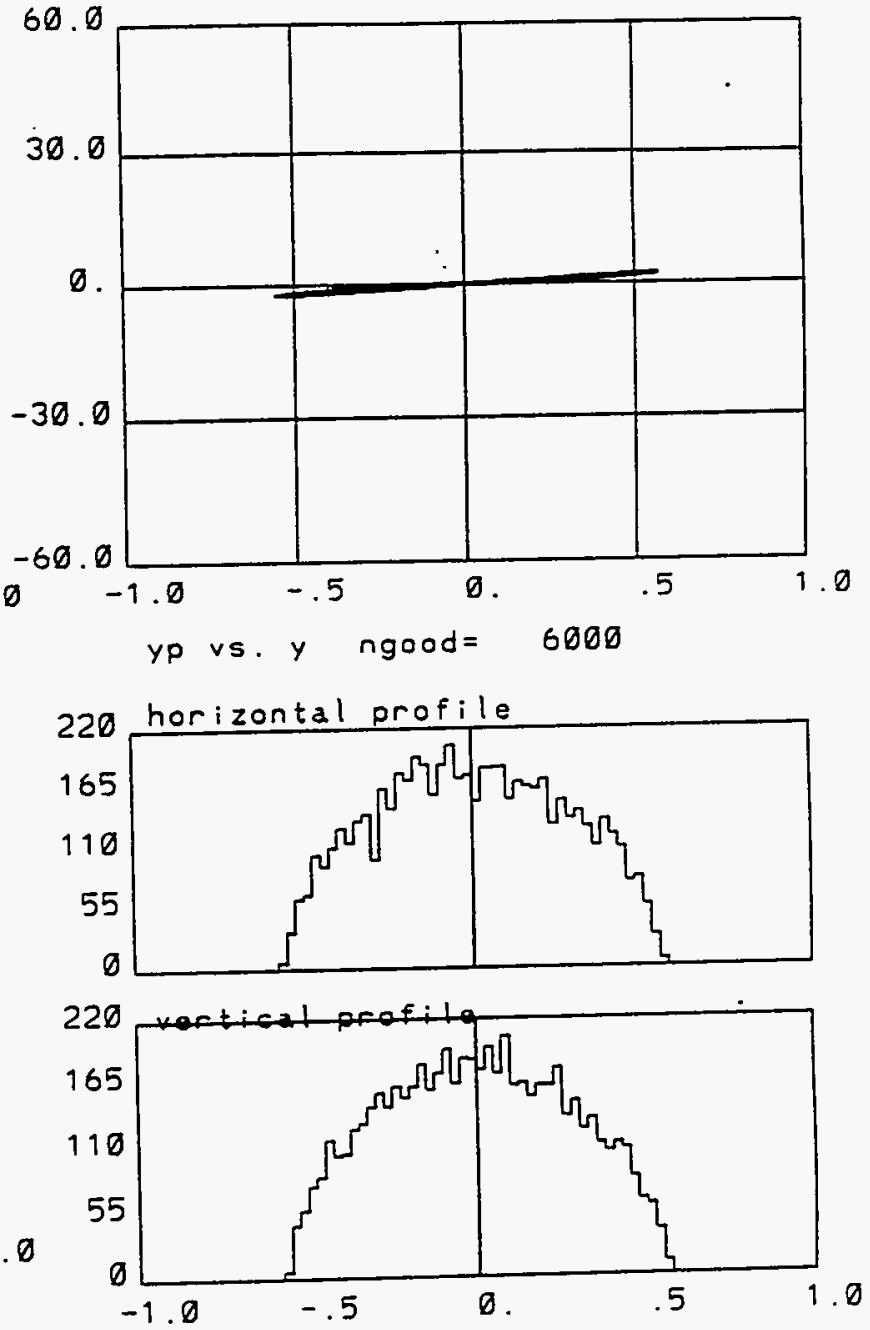
Figure $4-7$ shows the beam profile at position $(n e=20) \quad z=40$, with the Inline-Injection system of Solenoid + Gun + Solenoid combination inline with the linac. With Solenoid current of $I=2160 \mathrm{amp}$.

- BNL RF GUN
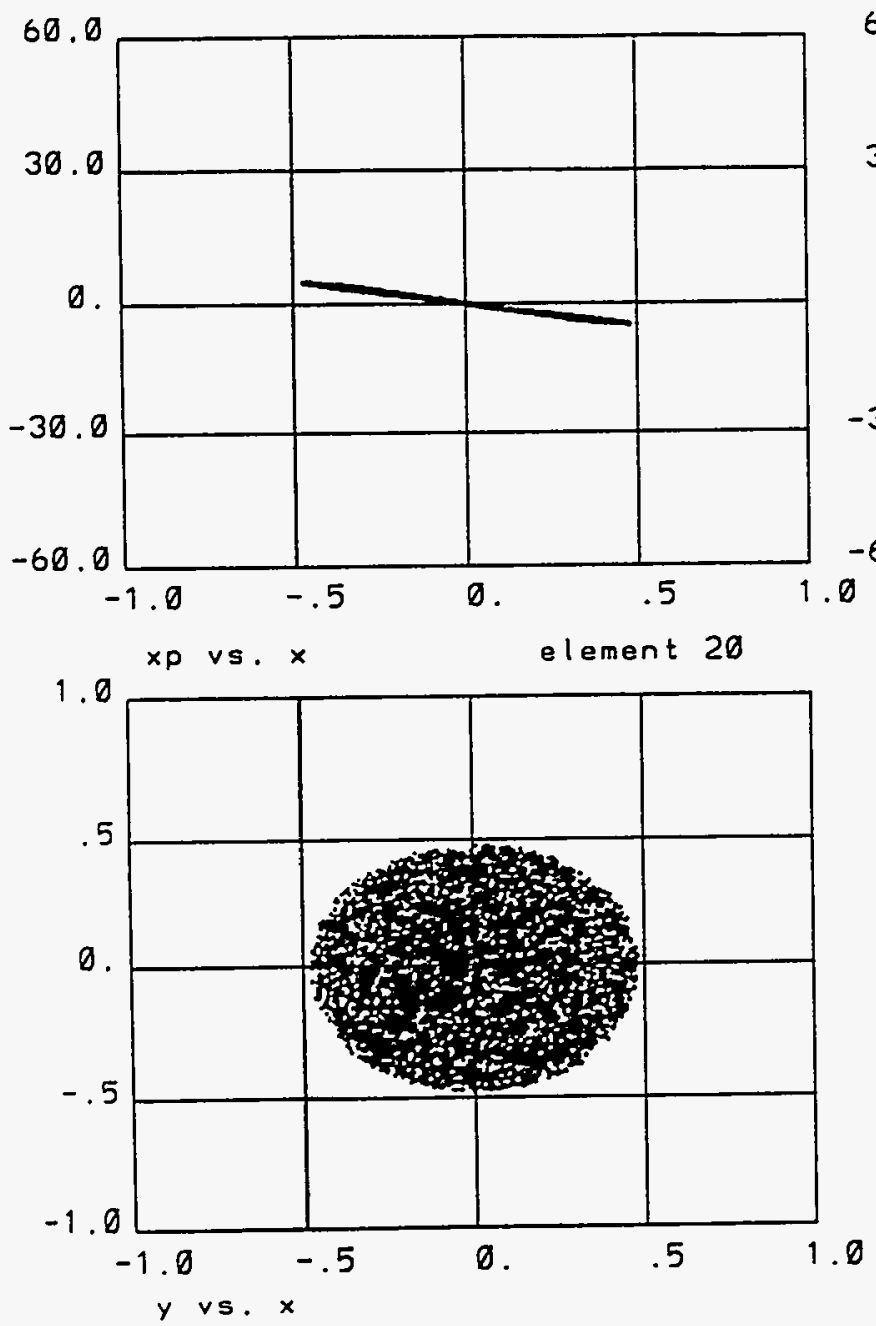

$5.15 p s$ G $(10), 1 n C$, phi $=43.1^{\circ}$

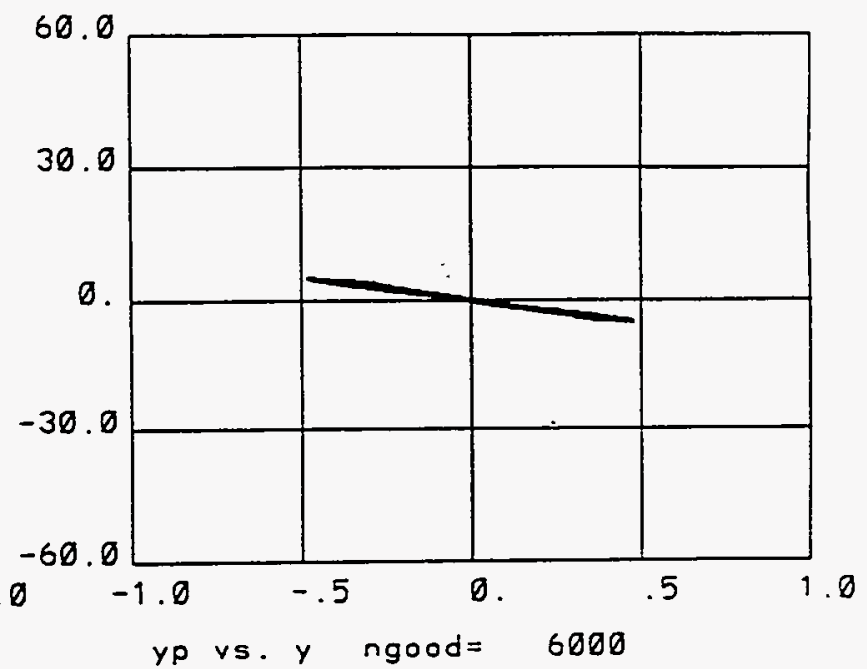

260 horizontal profile
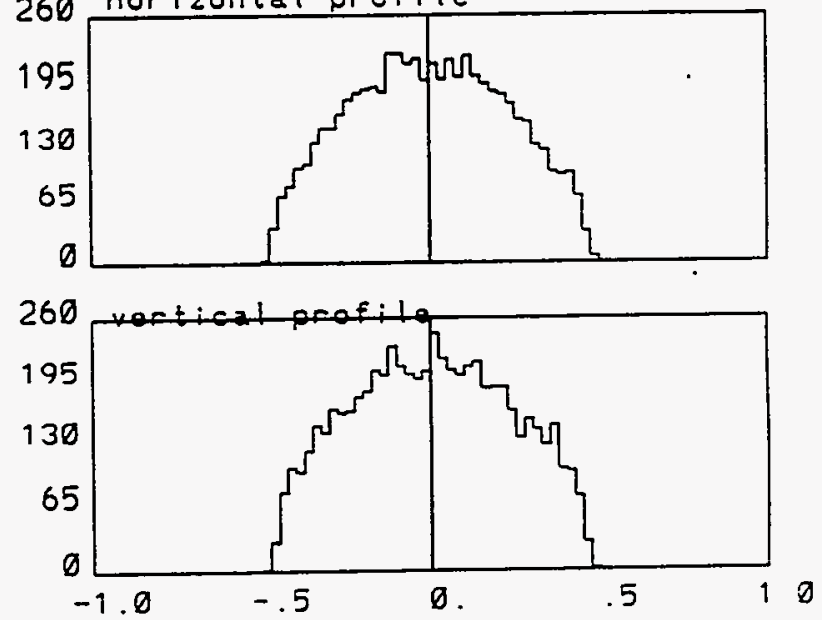
Figure $4-8$ shows the beam profile at position $(n e=30) z=70$, with the Inline-Injection system of Solenoid + Gun + Solenoid combination inline with the linac. With Solenoid current of $I=2160 \mathrm{amp}$.

'BNL RF GUN
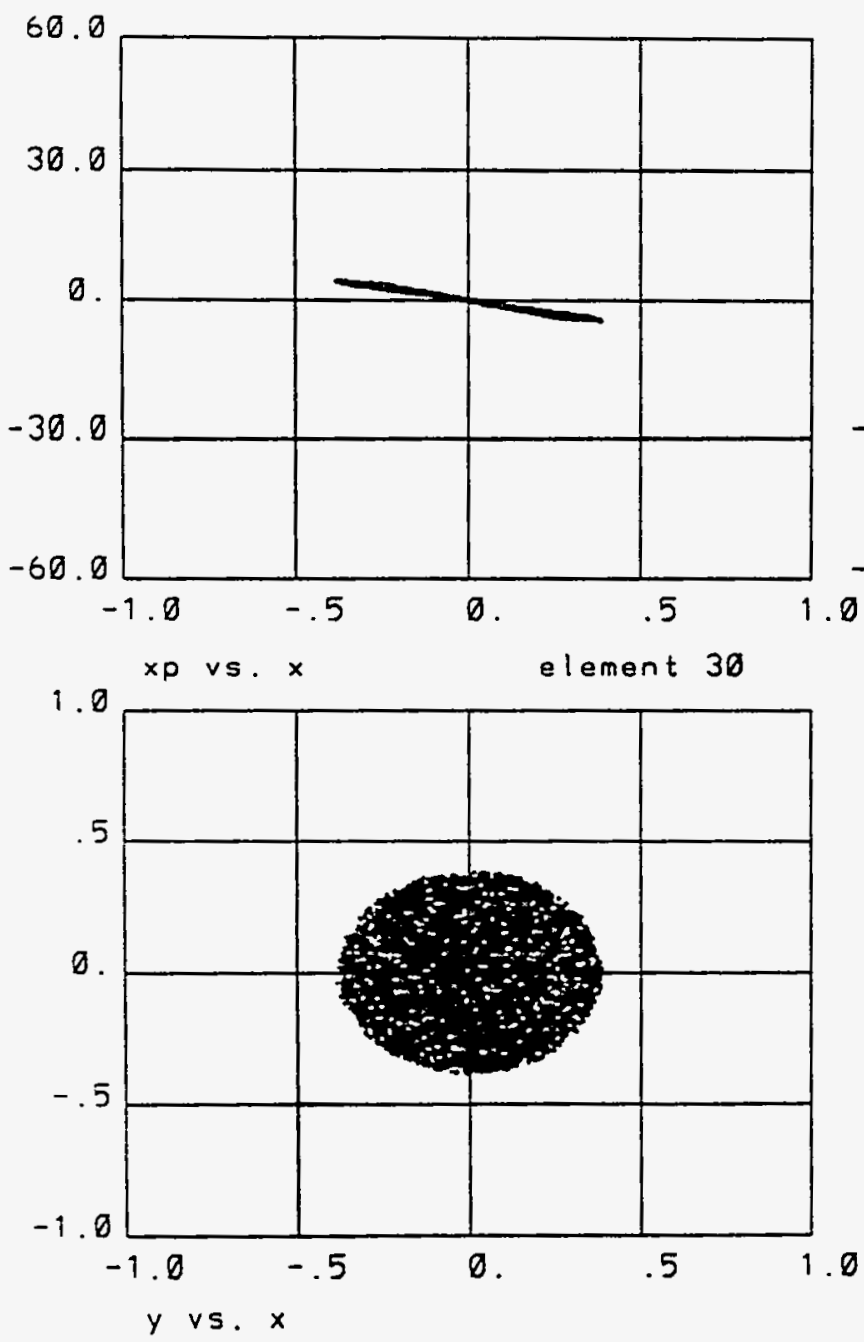

5.15os G(10), $1 n C$, ohi $=43.1^{\prime}$

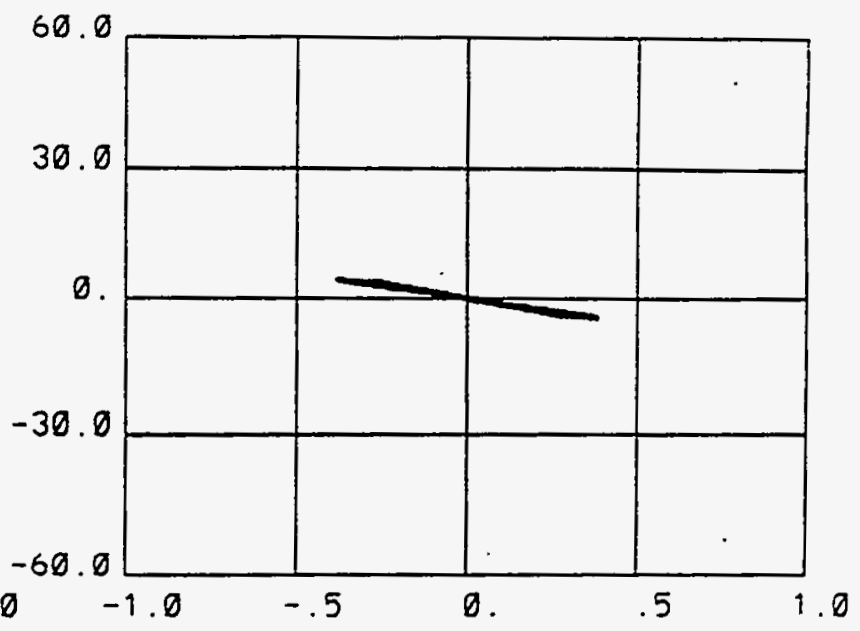

yp vs. y ngood $=6000$
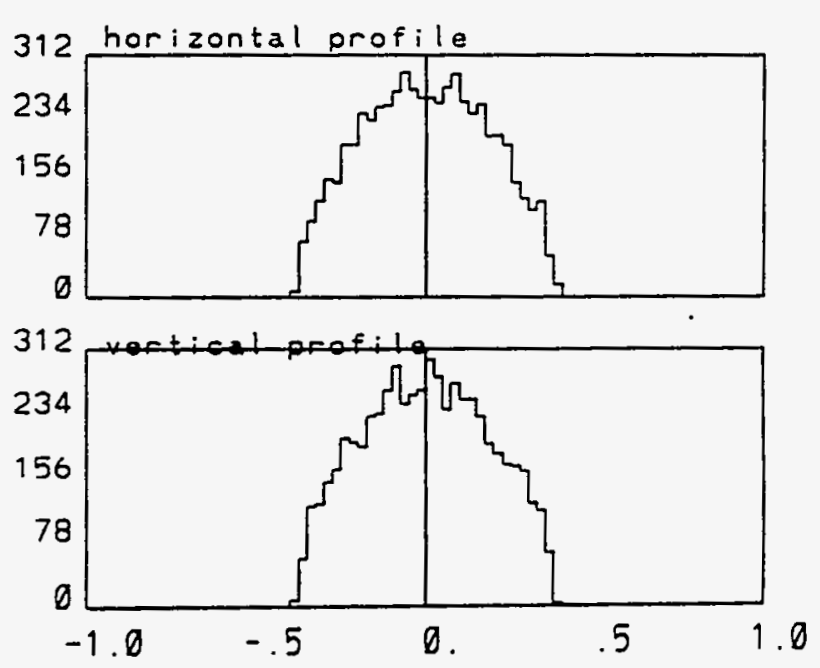
Figures 5-1 to 5-8 shows the beam profile at various positions (distance $z$ from the cathode), with the Inline-Injection system of Solenoid + Gun + Solenoid combination inline with the linac. With Solenoid current of I=2180 amp.

- BNL RF GUN
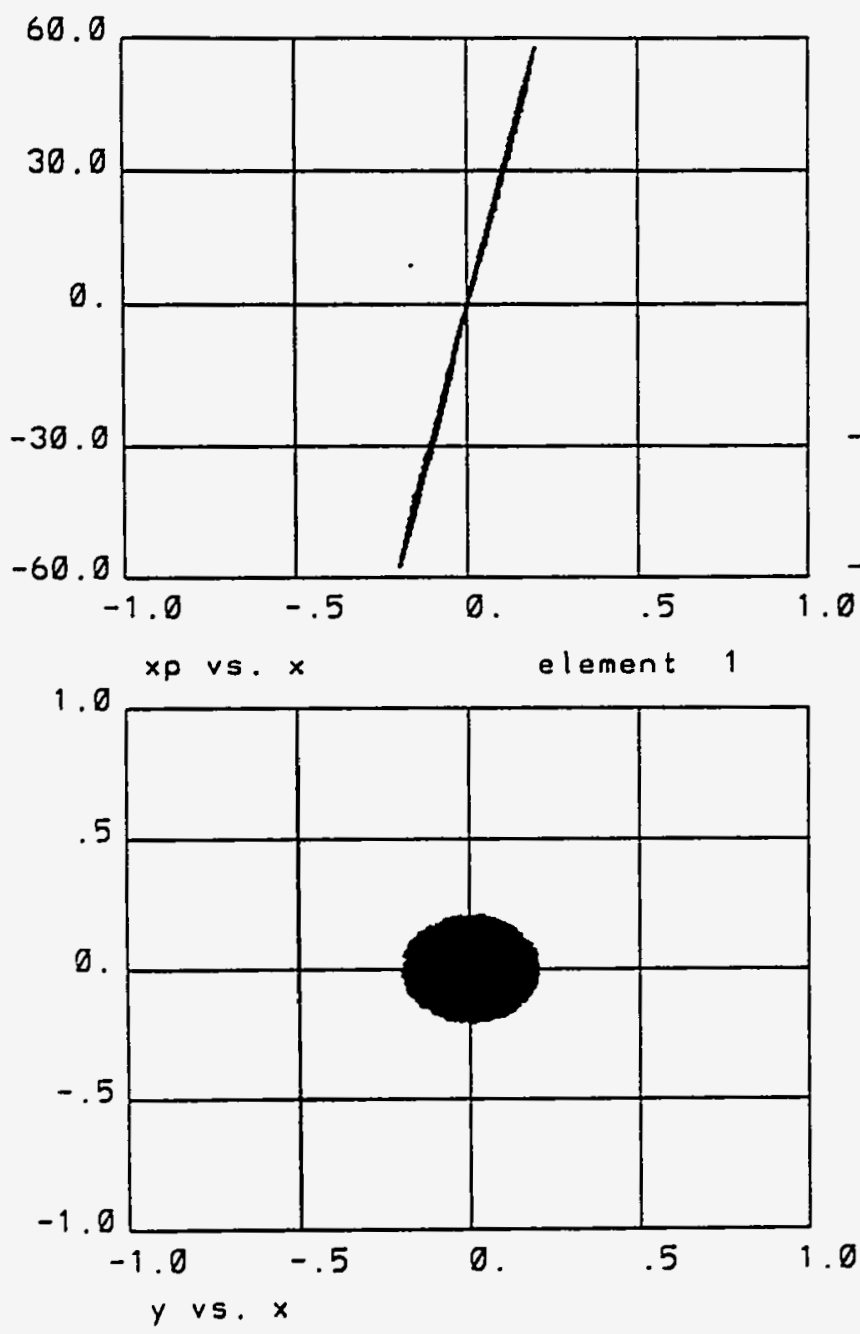

$5.15 p s G(10): 1 n C$, ohi $=43.)^{\circ}$

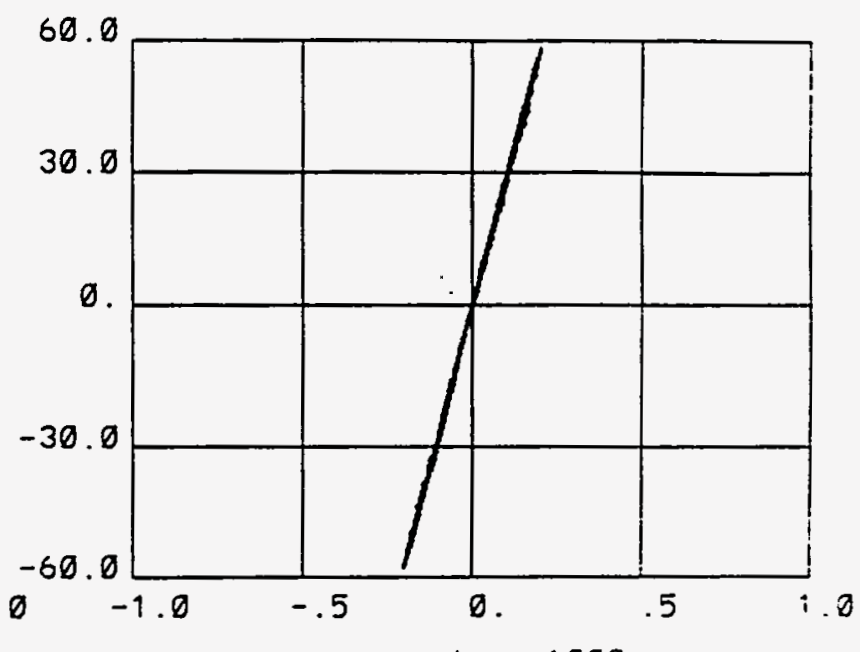

yp vs.y ngood $=6000$
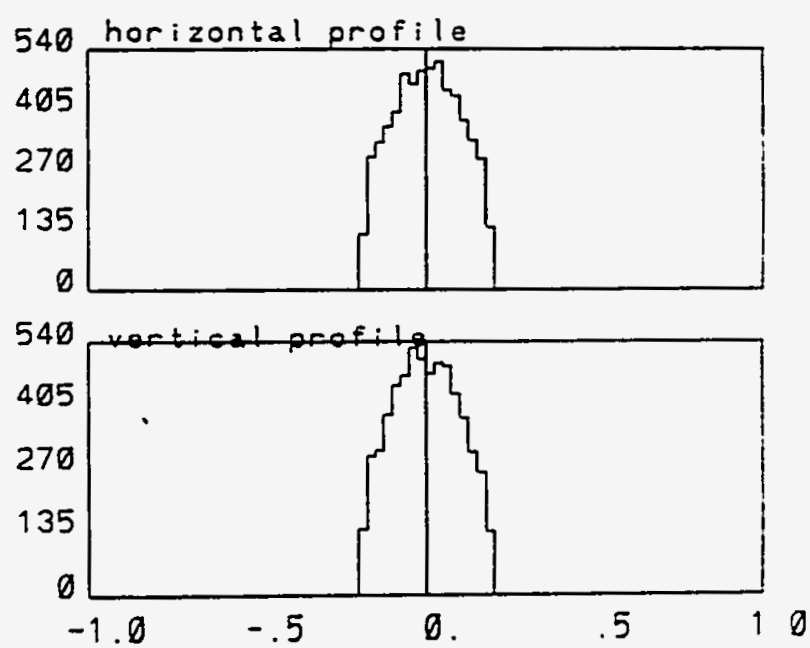

Figure $5-1$ shows the beam profile at position $(n e=1) \quad z=0$, with the Inline-Injection system of Solenoid + Gun + Solenoid combination inline with the linac. With Solenoid current of $I=2180 \mathrm{amp}$. 
Figure $5-2$ shows the beam profile at position $(n e=2) z=2.6$, with the Inline-Injection system of Solenoid + Gun + Solenoid combination inline with the linac. With Solenoid current of $I=2180 \mathrm{amp}$.

- BNL RF GUN
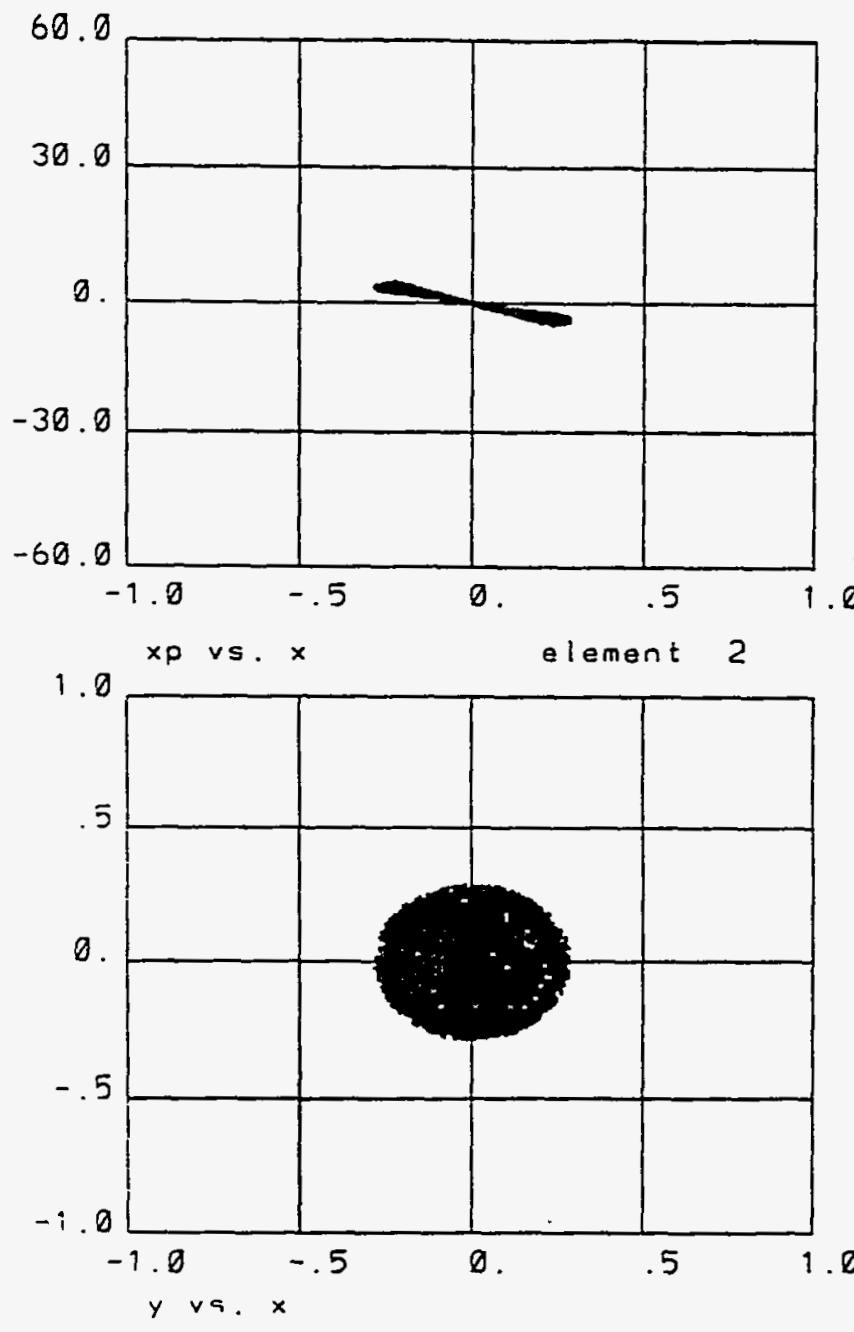

5. $15 p s G(10), 1 n C, p h i=43.1^{\circ}$

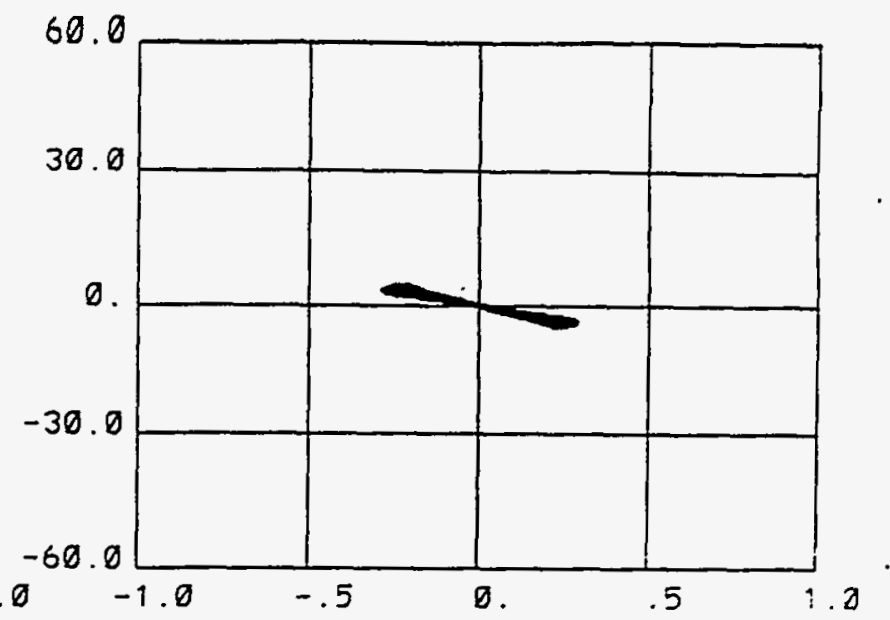

yp vs. y ngood $=6000$
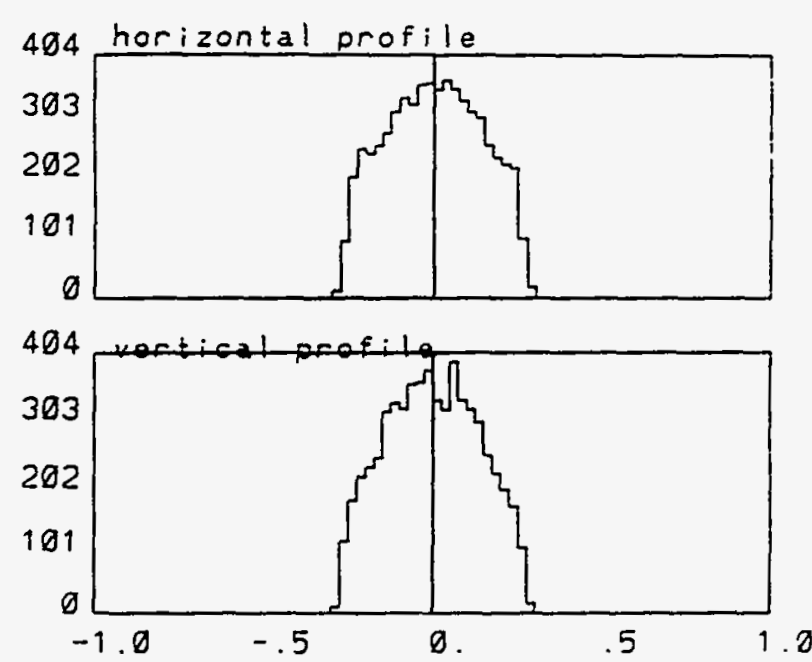
Figure $5-3$ shows the beam profile at position $(n e=3) z=5.2$, with the Inline-Injection system of Solenoid + Gun + Solenoid combination inline with the linac. With Solenoid current of $I=2180 \mathrm{amp}$.

- BNL RF GUN
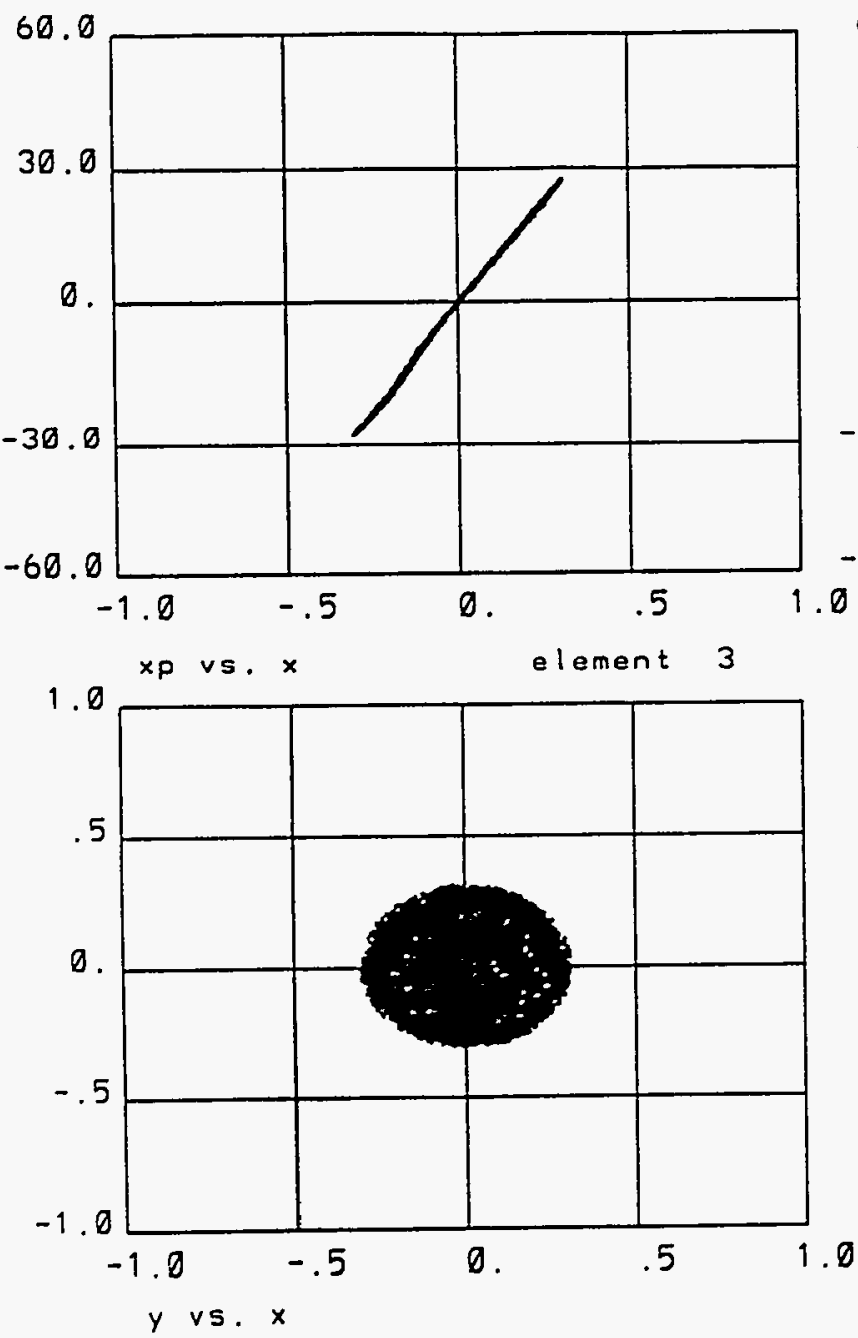

5.15ps $G(10) .1 n C$, phi $=43.1$.
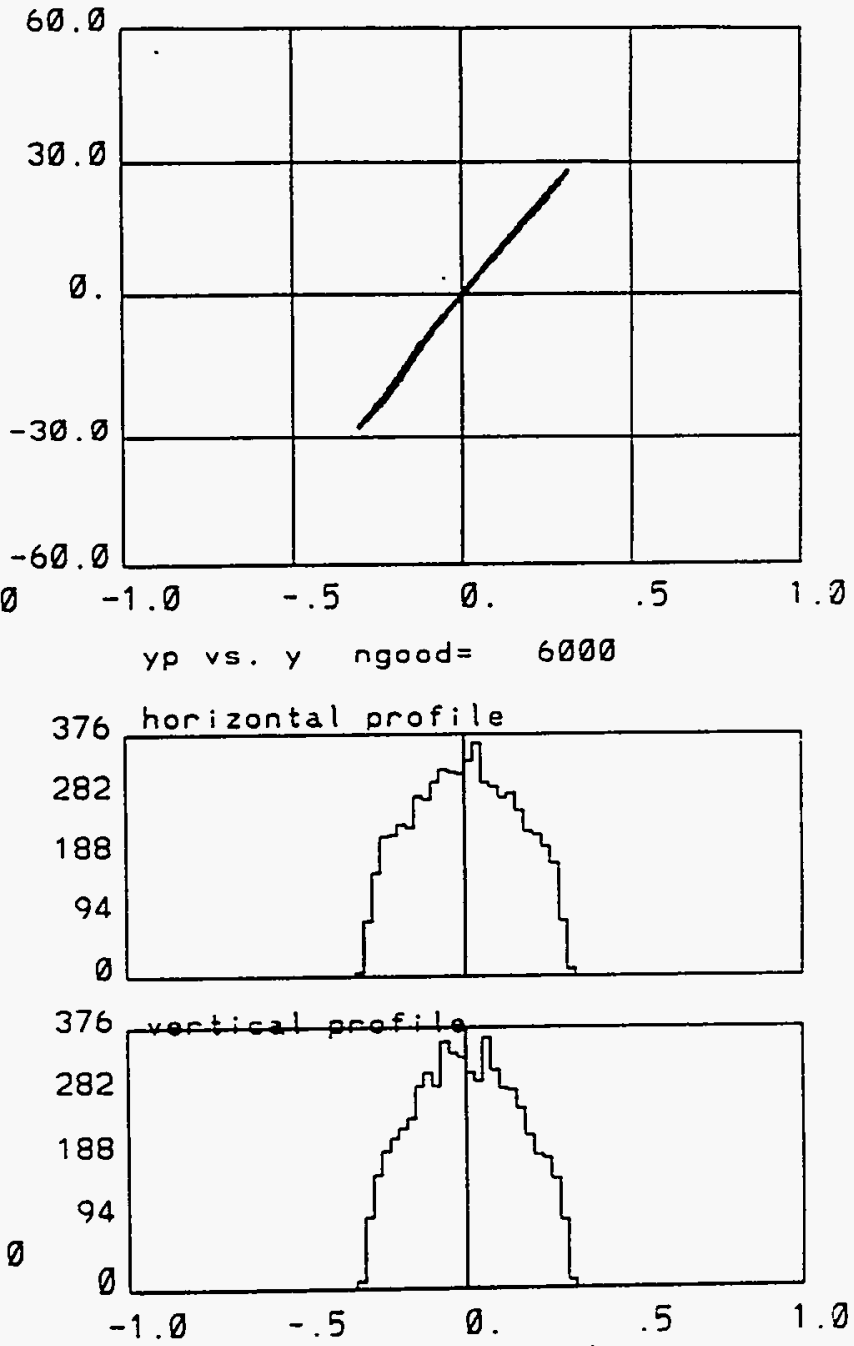
Figure $5-4$ shows the beam profile at position $(n e=4) z=7.9$, with the Inline-Injection system of Solenoid + Gun + Solenoid combination inline with the linac. With Solenoid current of $I=2180$ amp.

'BNL RF GUN

60.0

30.0

$\emptyset$

$-30$

$-60.0$
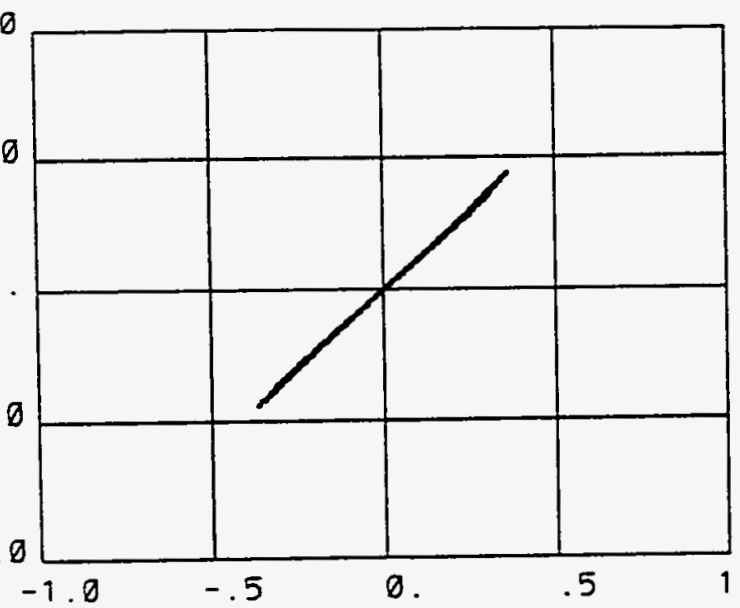

$x p \vee s . x$

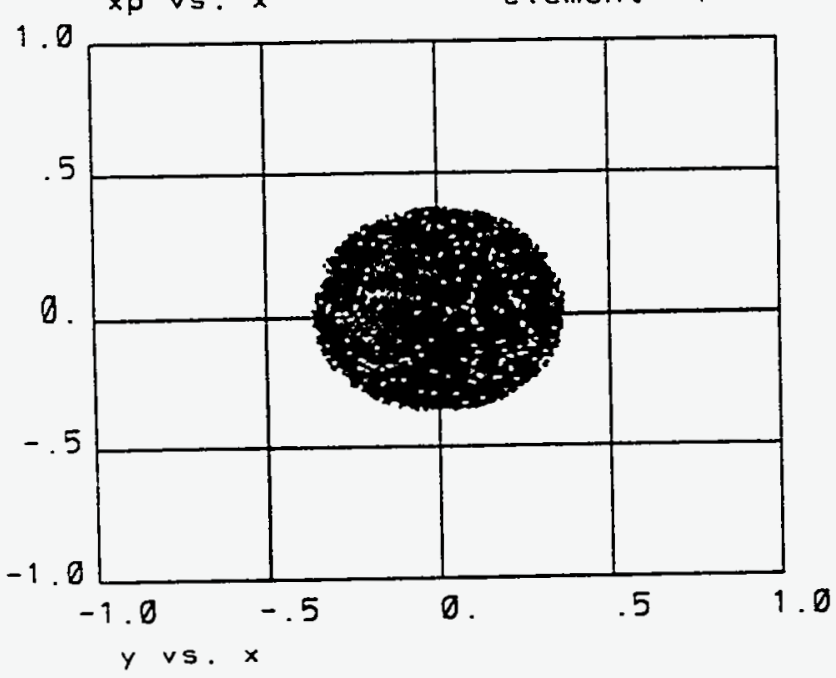

$5.15 p s \mathrm{G}(10), 1 n C$, phi $=43.)^{\circ}$
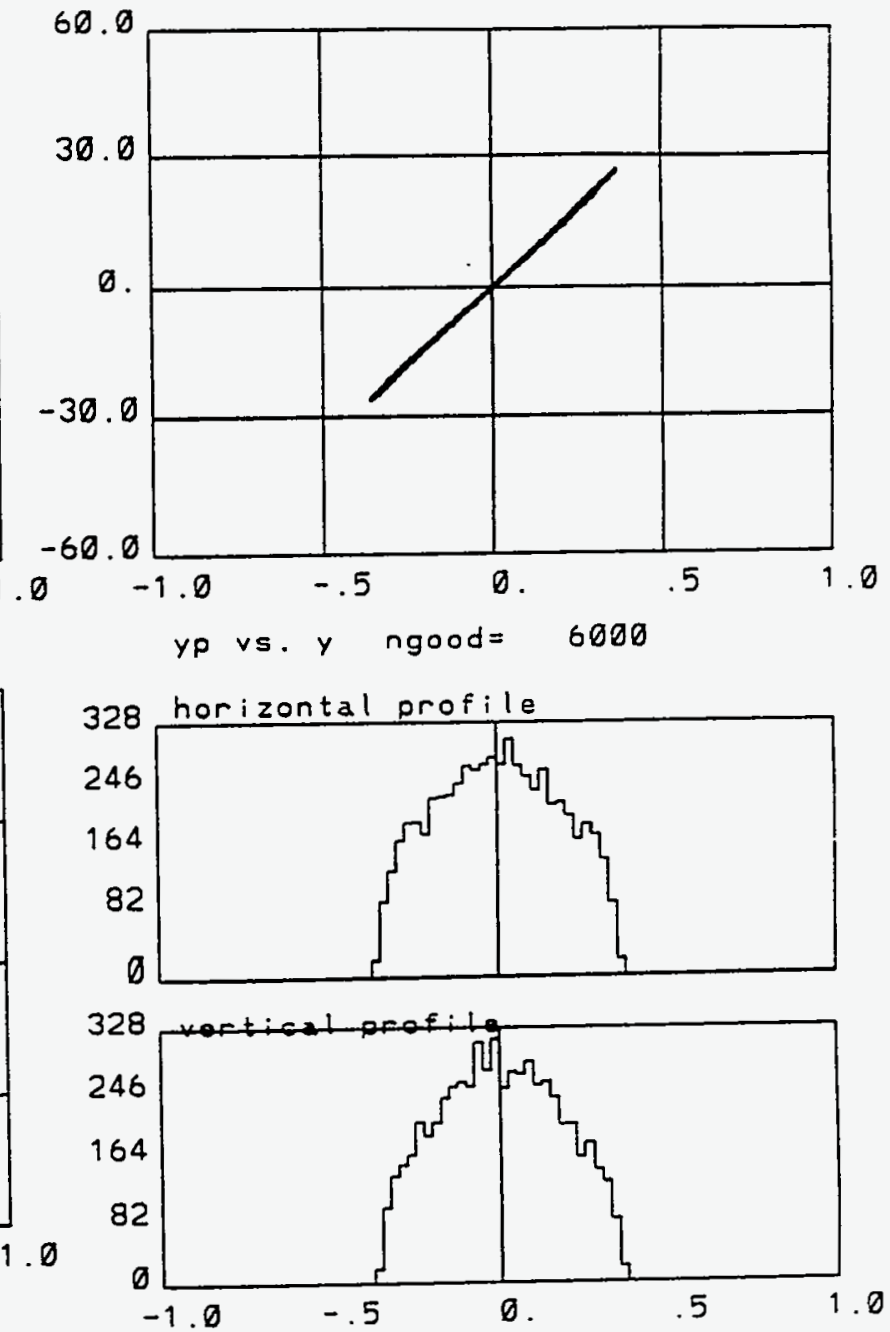
Figure $5-5$ shows the beam profile at position $(n e=5) z=10$, with the Inline-Injection system of Solenoid + Gun + Solenoid combination inline with the linac. With Solenoid current of $\mathrm{I}=\mathbf{2 1 8 0} \mathrm{amp}$.

'BNL RF GUN
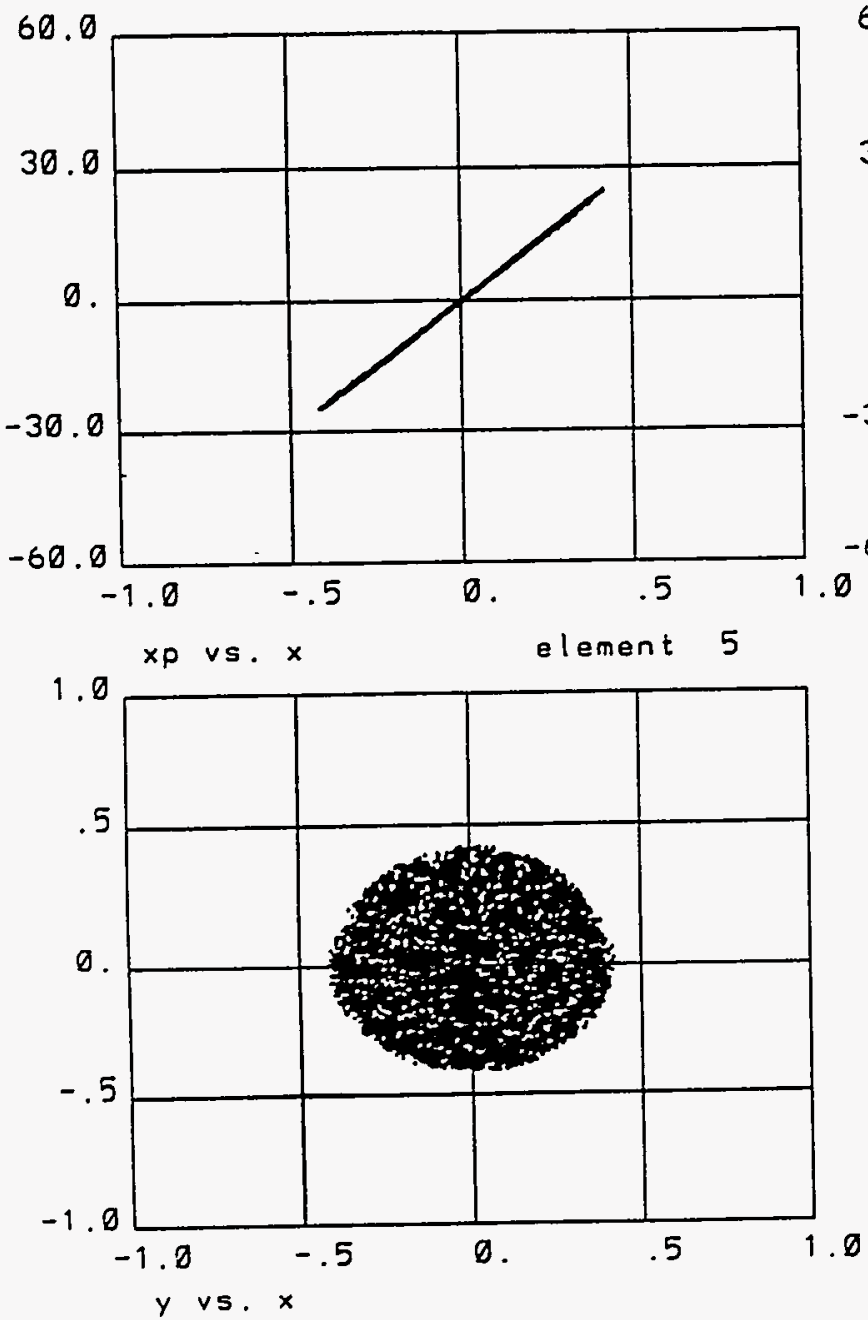

$5.15 p s$ G $(10), 1 n C_{1}$ phi $=43.1^{\circ}$

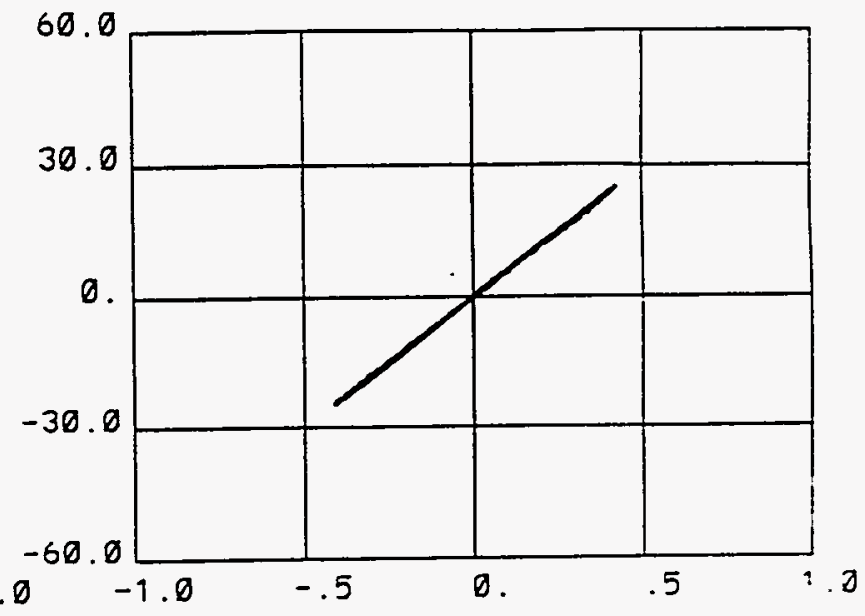

yp vs.y ngood $=6000$
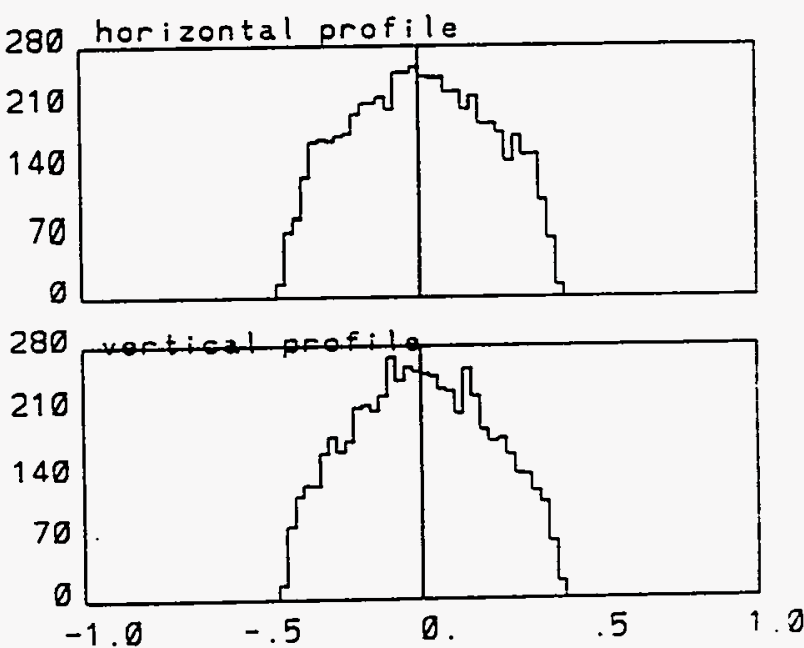
Figure 5 -6 shows the beam profile at position $(n e=10) z=20$, with the Inline-Injection system of Solenoid + Gun + Solenoid combination inline with the linac. With Solenoid current of $I=2180 \mathrm{amp}$.

- BNL RF GUN
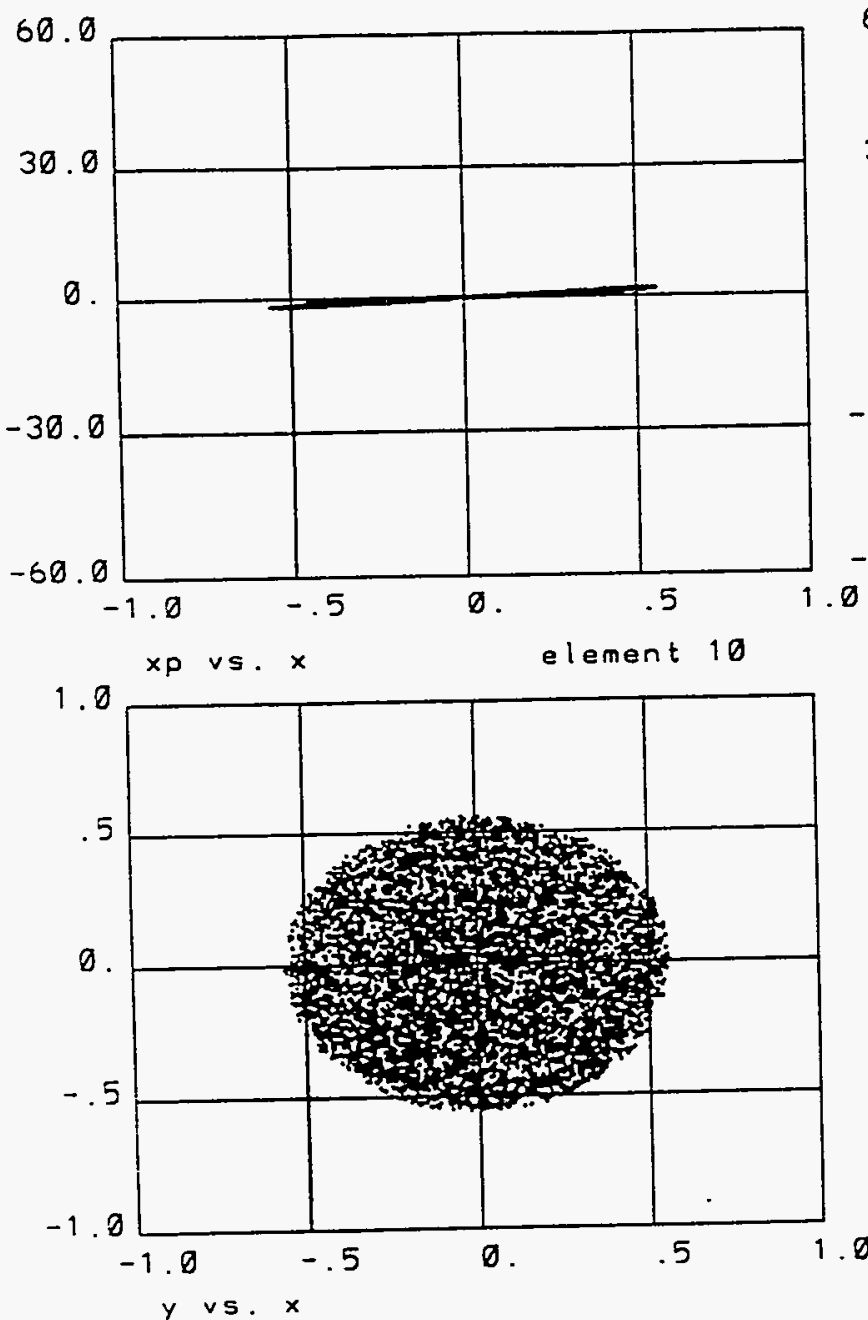

15.15ps $G(10), 1$ nC, ohi $=43$.
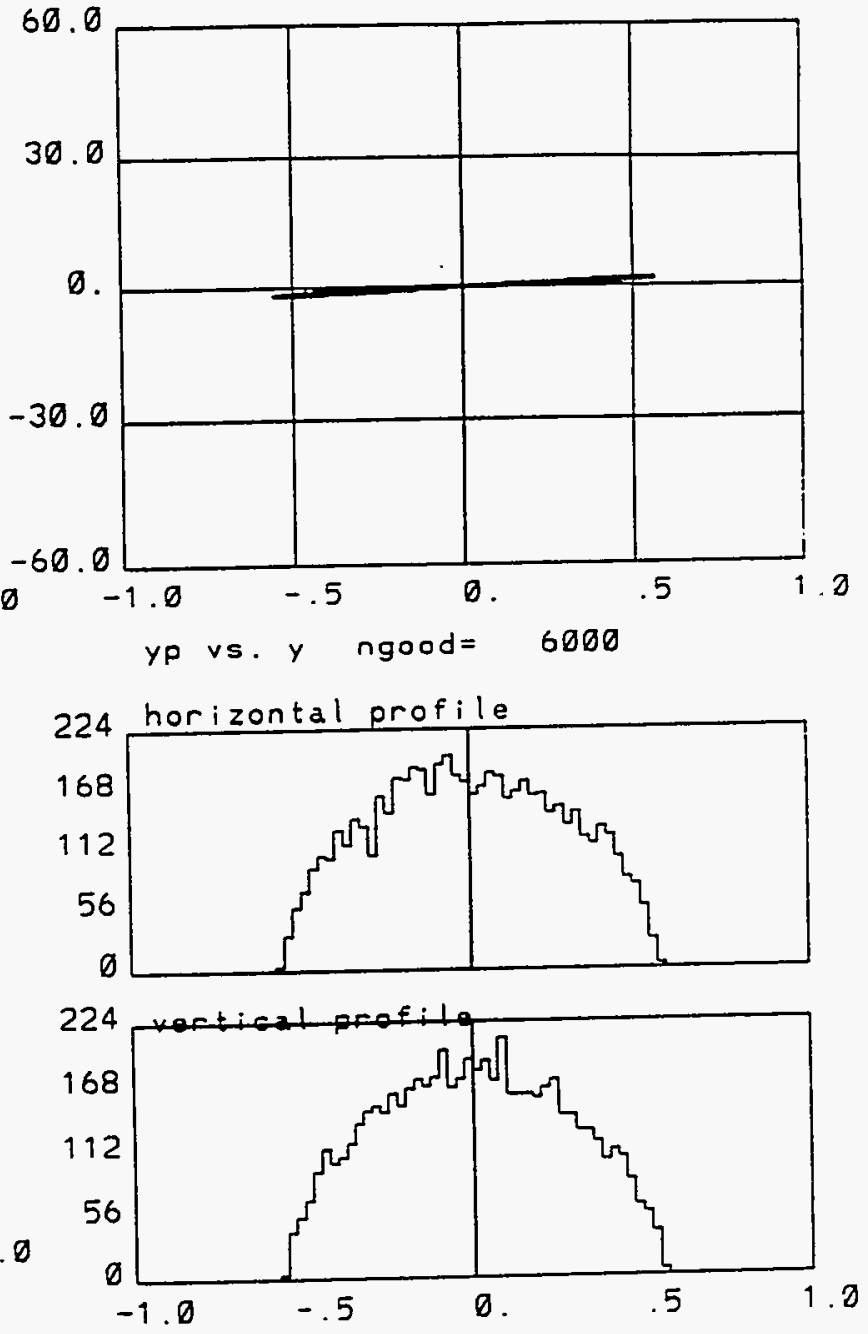
Figure $5-7$ shows the beam profile at position $(\dot{n} e=20) z=40$, with the Inline-Injection system of Solenoid + Gun + Solenoid combination inline with the linac. With Solenoid current of $I=2180 \mathrm{amp}$.

- BNL RF GUN

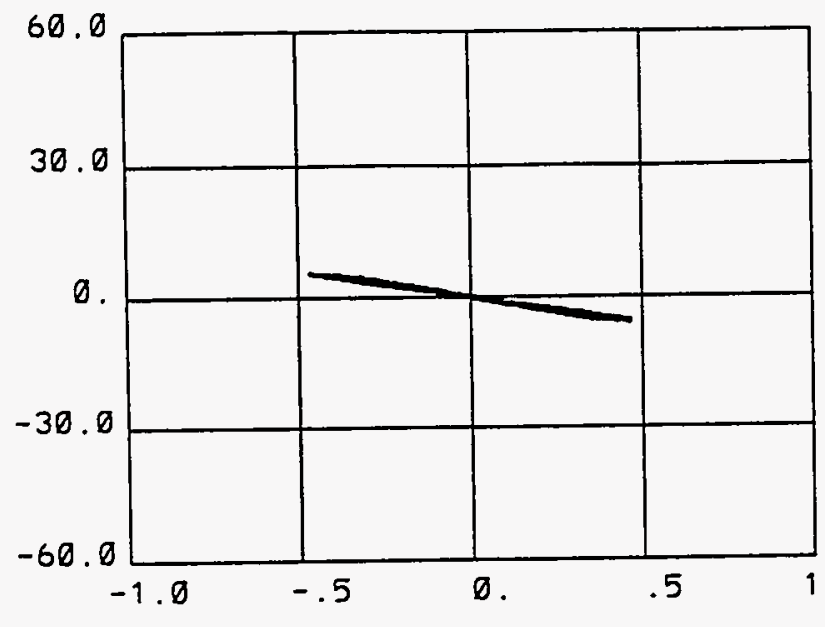

xp vs. $x$ element 20

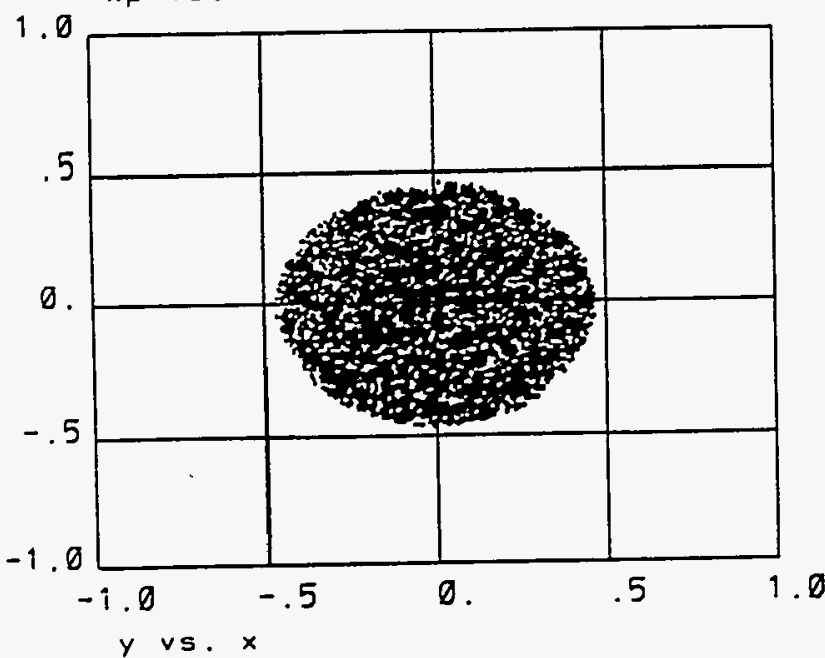

15.15ps G(10),1 nC, phi=43.1.
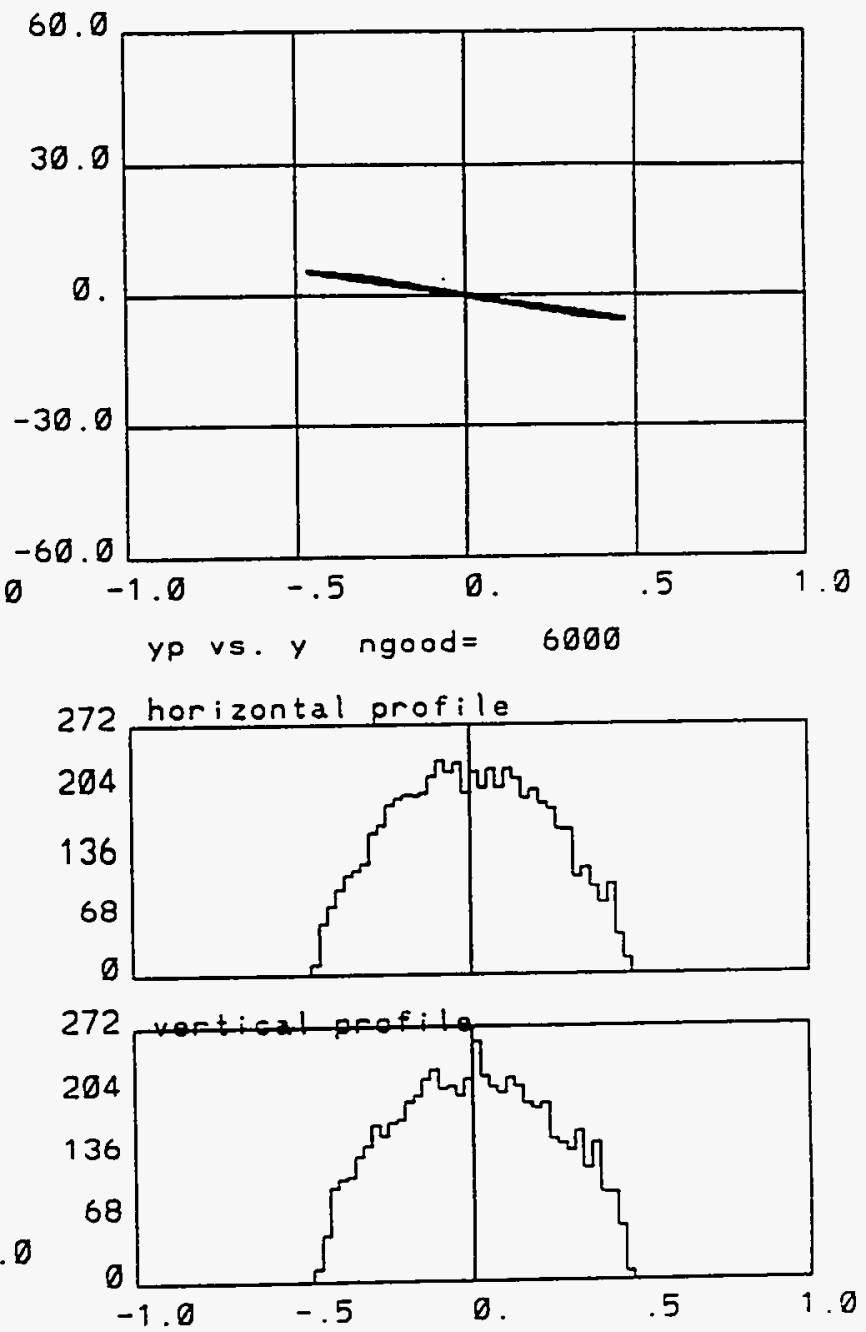
Figure $5-8$ shows the beam profile at position $(n e=30) z=70$, with the Inline-Injection system of Solenoid + Gun + Solenoid combination inline with the linac. With Solenoid current of $\mathrm{I}=2180 \mathrm{amp}$.

-BNL RF GUN
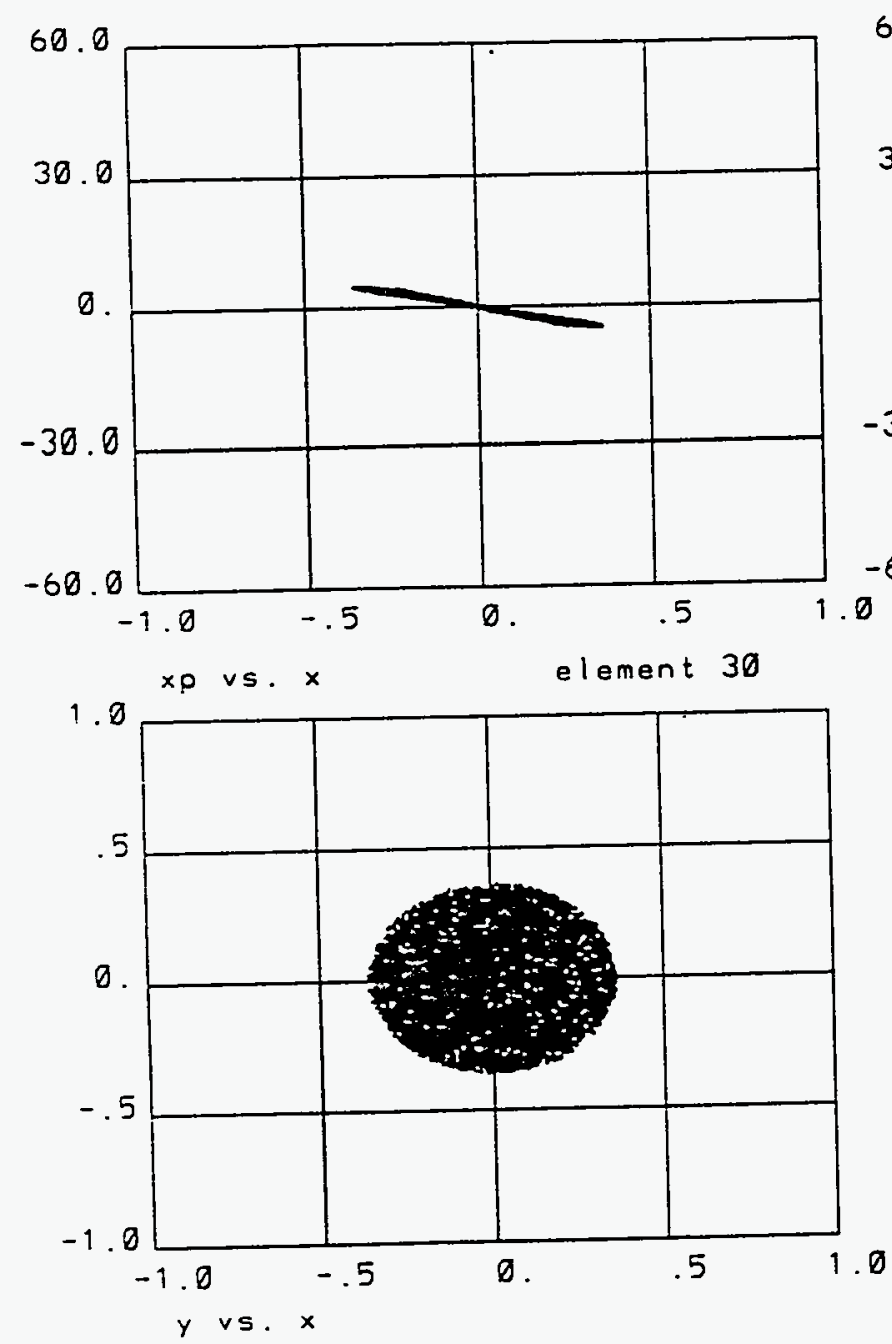

,5.15ps $G(10), 1 \mathrm{nC}$, phi $=43.1^{\circ}$
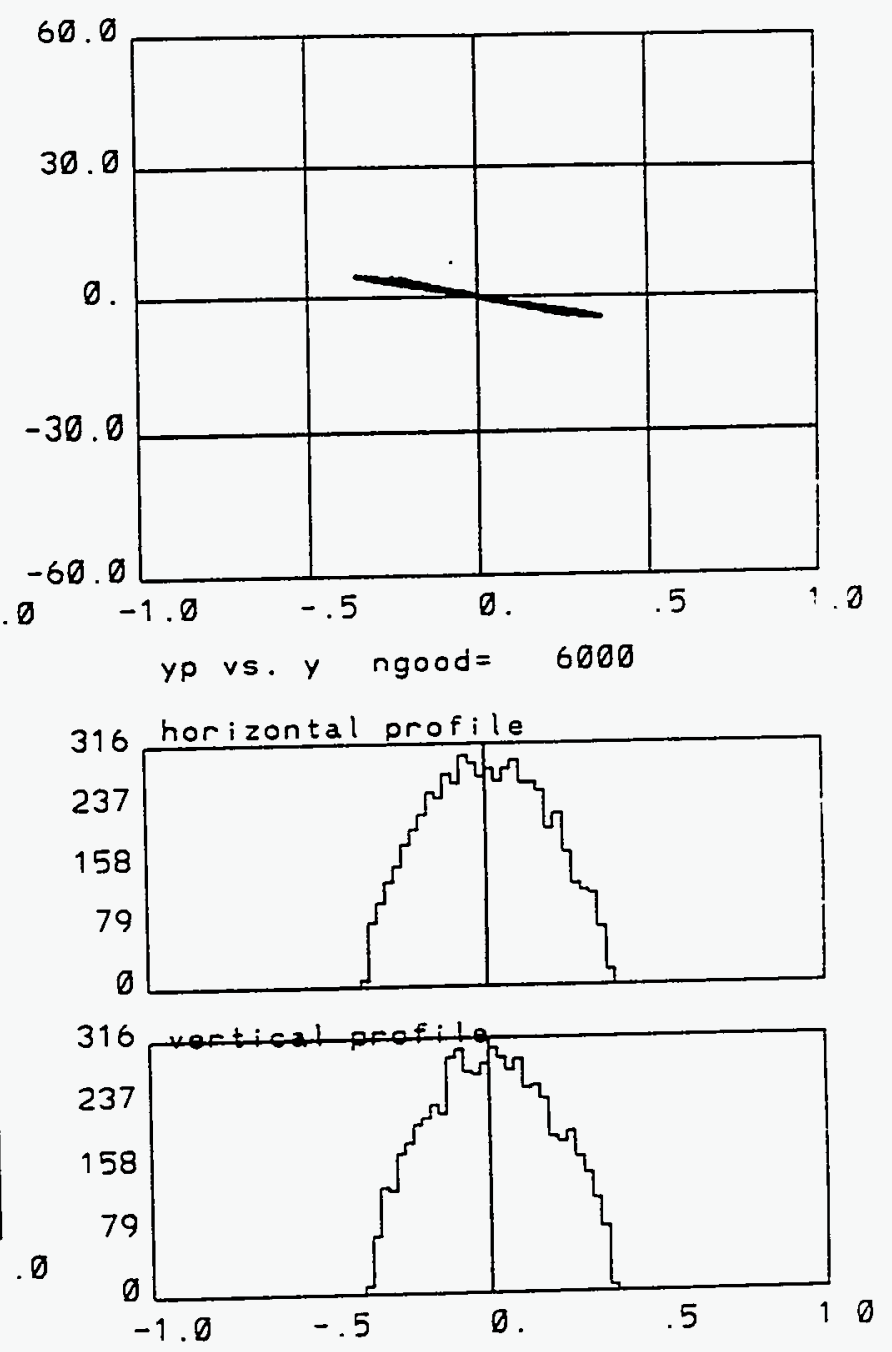
Figures $6-1$ to 6-8 shows the beam profile at various positions (distance $z$ from the cathode), with the Inline-Injection system of Solenoid + Gun + Solenoid combination inline with the linac. With Solenoid current of I=2200 $\mathbf{a m p}$.

- BNL RF GUN

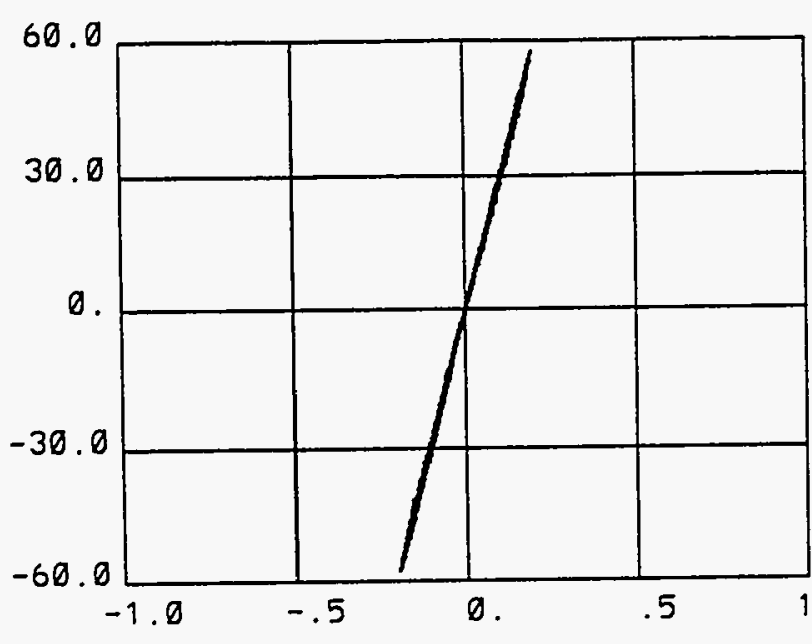

$x p \vee s . x$

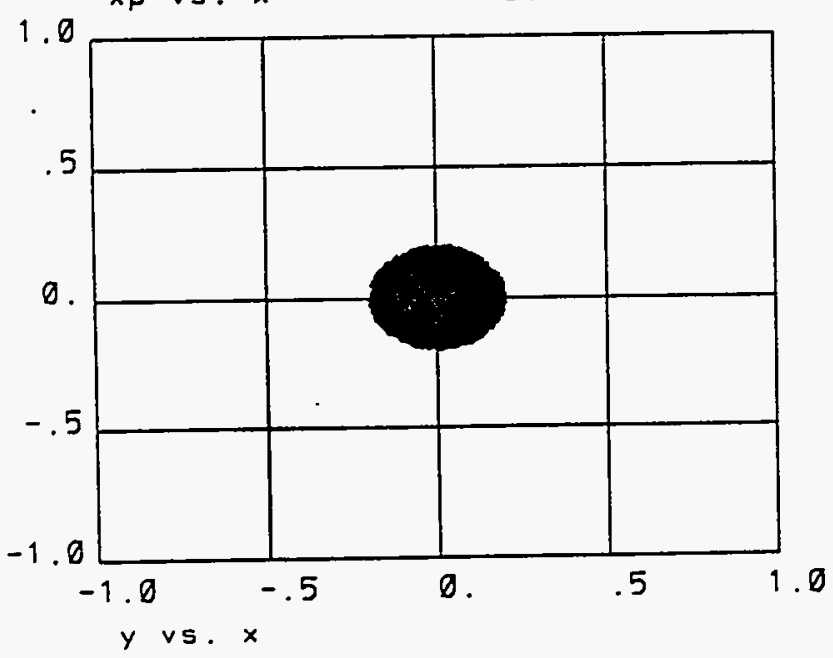

5.15ps $G(10) .1 \mathrm{nC}$, phi=43.).

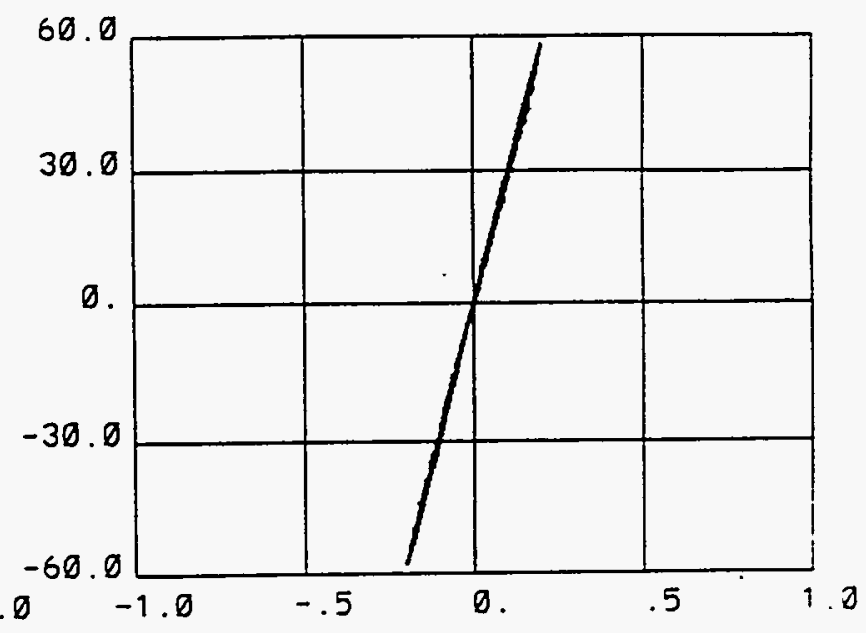

yp vs. y ngood $=6000$
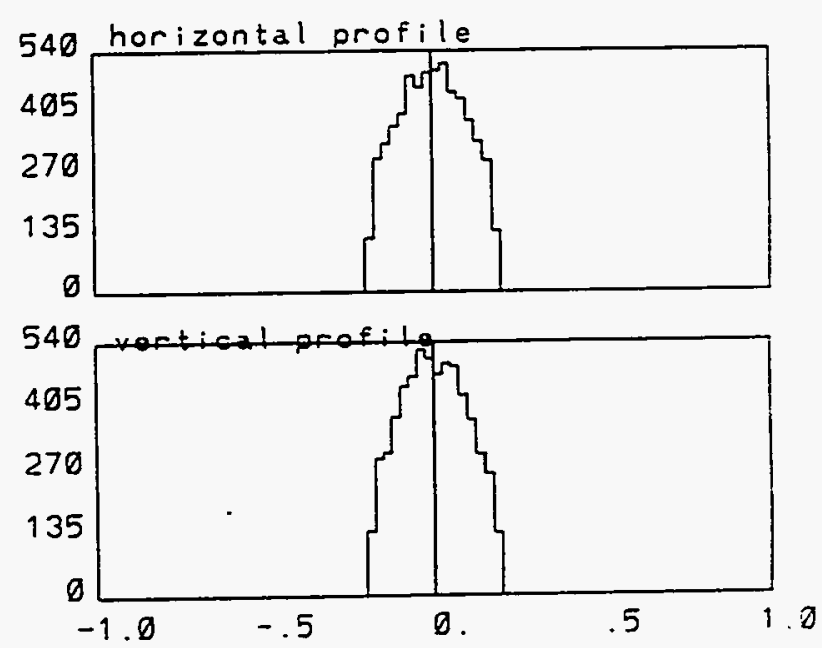

Figure 6-1 shows the beam profile at position $(n e=1) z=0$, with the Inline-Injection system of Solenoid + Gun + Solenoid combination inline with the linac. With Solenoid current of $I=2200 \mathrm{amp}$. 
Figure $6-2$ shows the beam profile at position $(n e=2) z=2.6$, with the Inline-Injection system of Solenoid + Gun + Solenoid combination inline with the linac. With Solenoid current of $I=2200 \mathrm{amp}$.

- BNL RF GUN
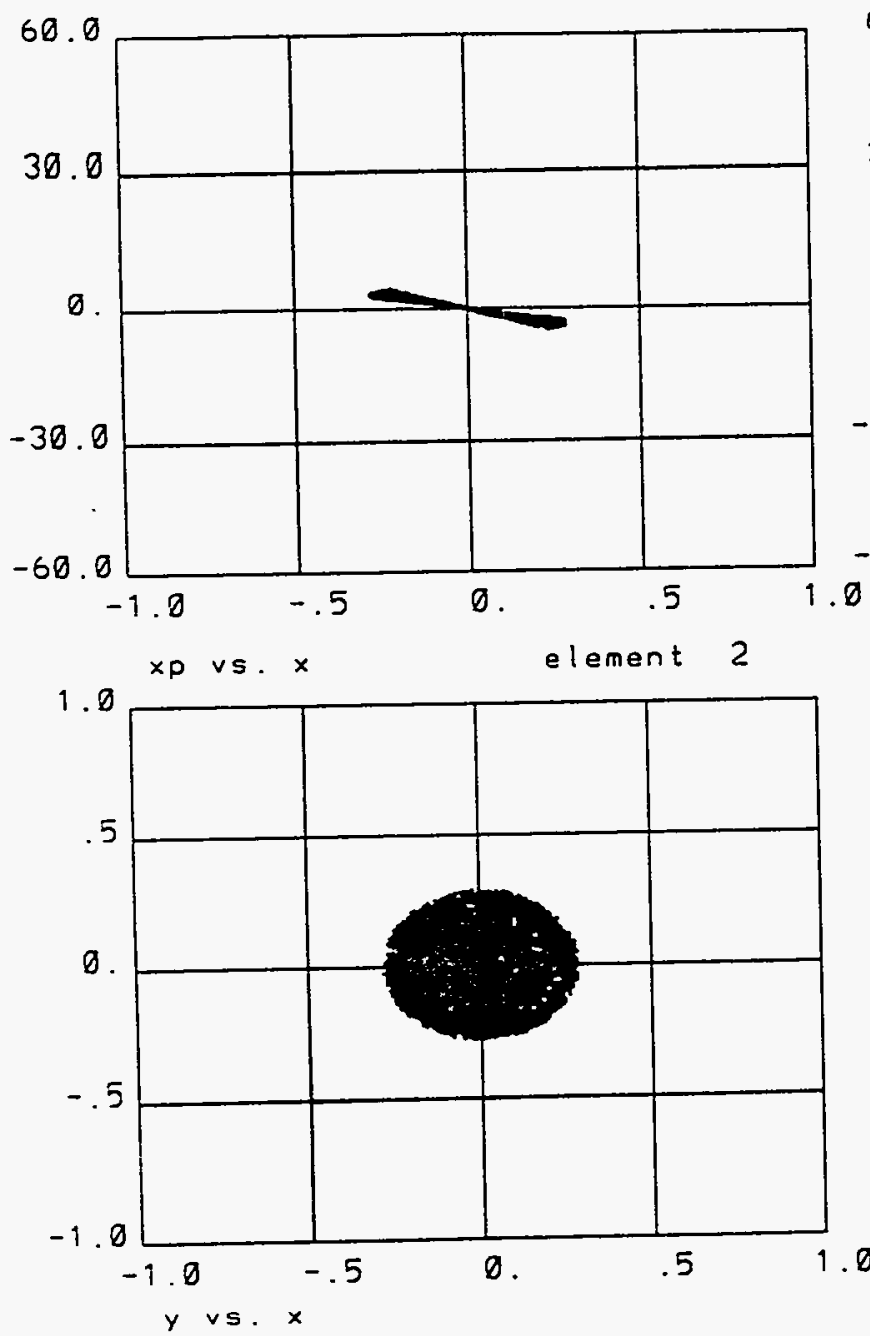

$15.15 p s \mathrm{~g}(10), 1 \mathrm{nC}, \mathrm{phi}=43$.$) .$
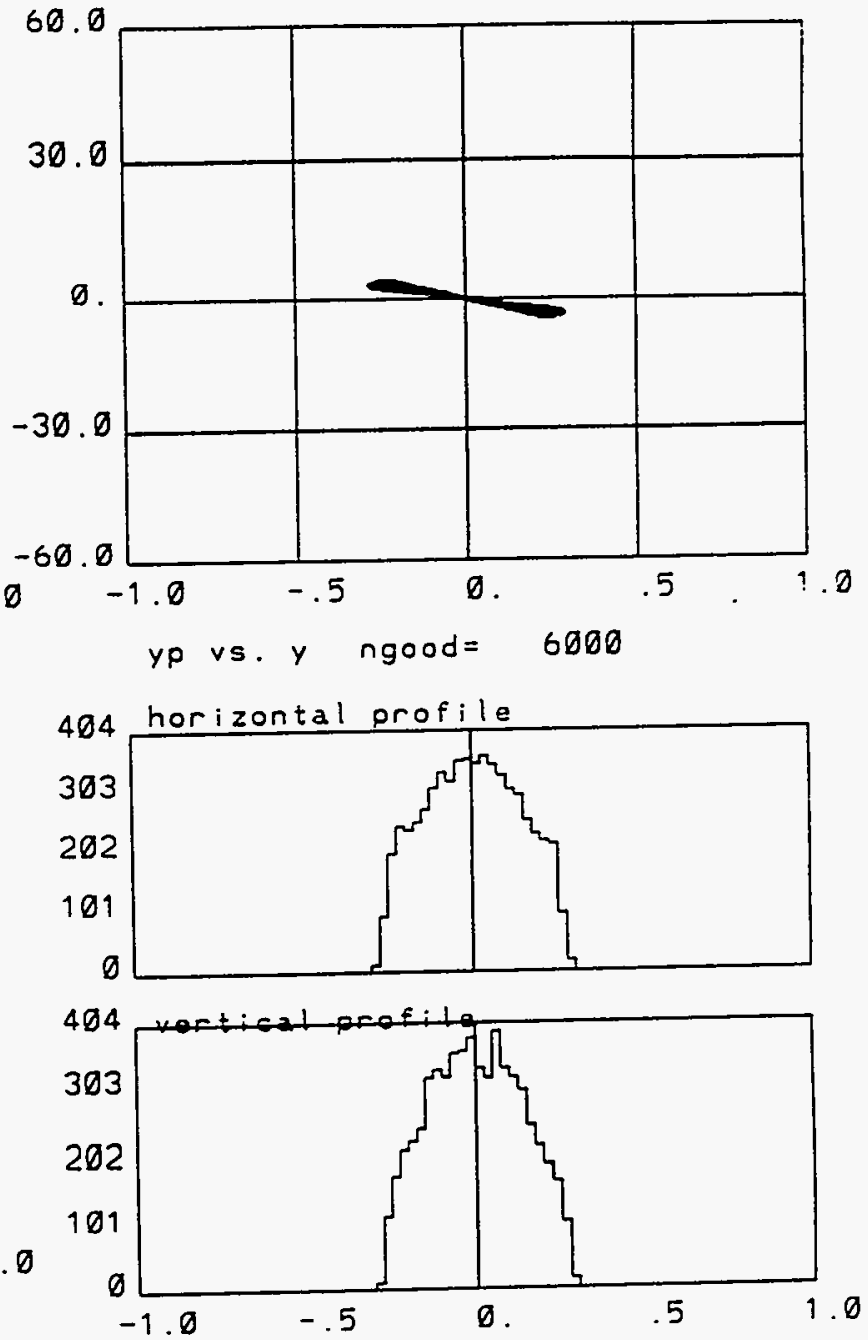
Figure $6-3$ shows the beam profile at position $(n e=3) \quad z=5.2$, with the Inline-Injection system of Solenoid + Gun + Solenoid combination inline . with the linac. With Solenoid current of $I=2200 \mathrm{amp}$.

- BNL RF GUN
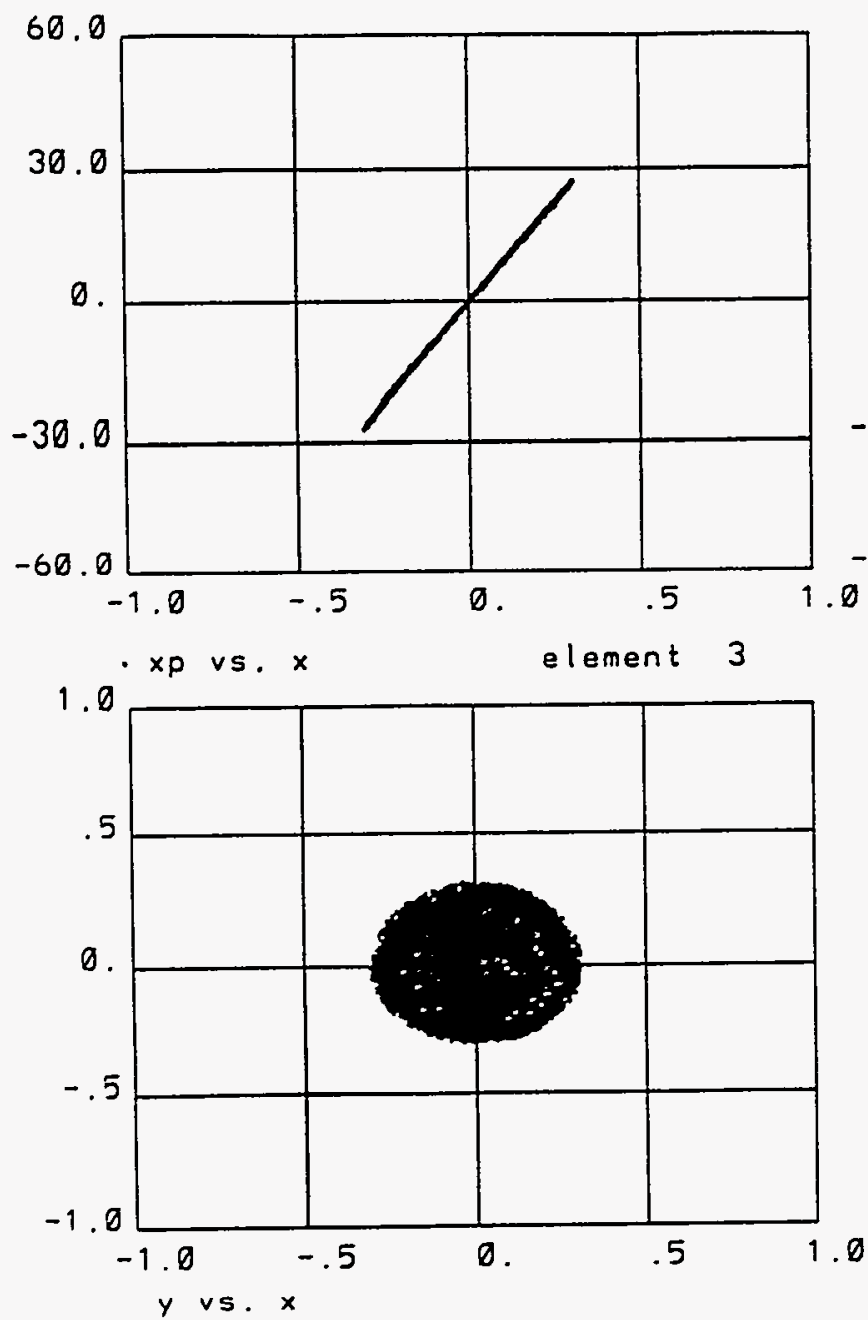

$5.15 p s G(10), 1$ nC, phi $=43.1$
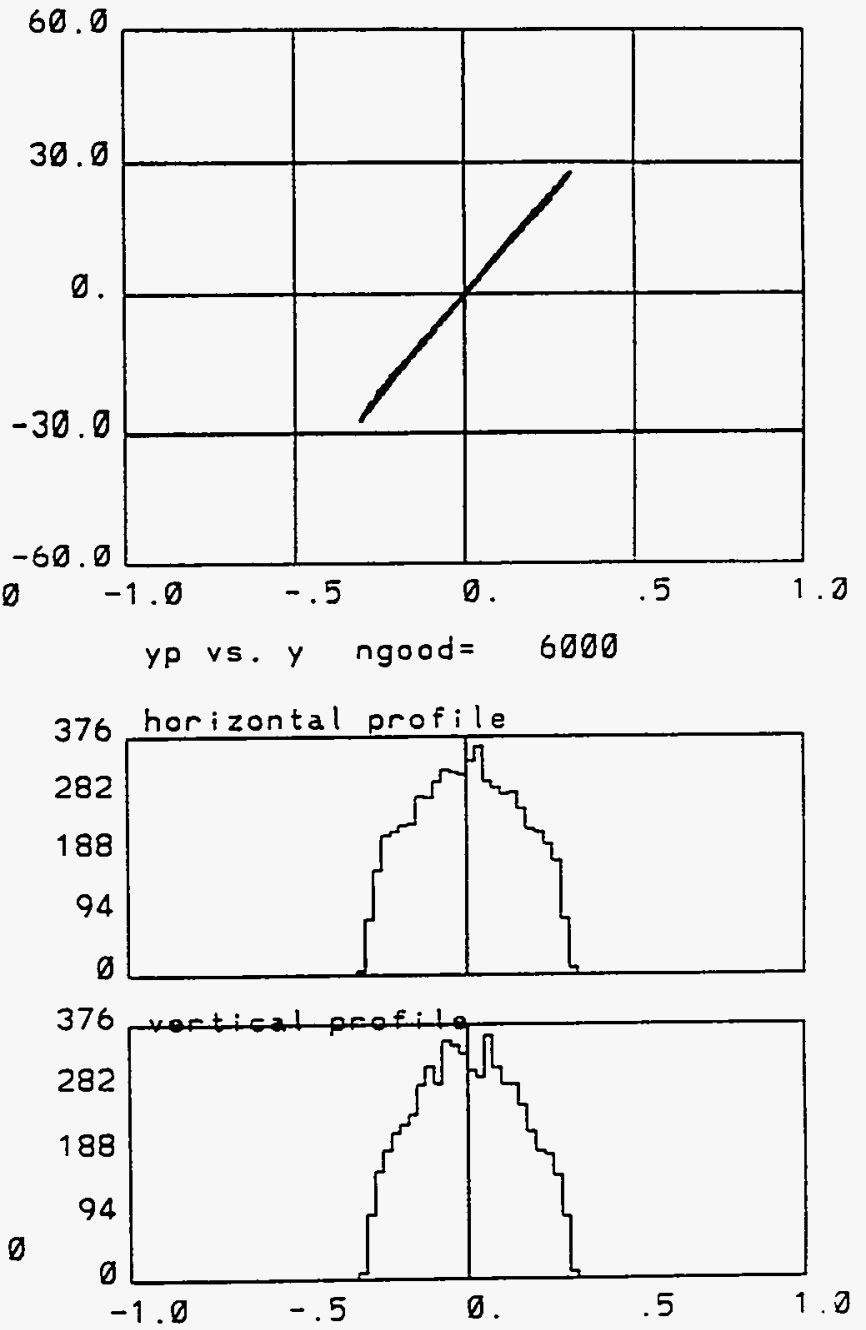
Figure $6-4$ shows the beam profile at position $(n e=4) z=7.9$, with the Inline-Injection system of Solenoid + Gun + Solenoid combination inline with the linac. With Solenoid current of $I=2200$ amp.

- BNL RF GUN
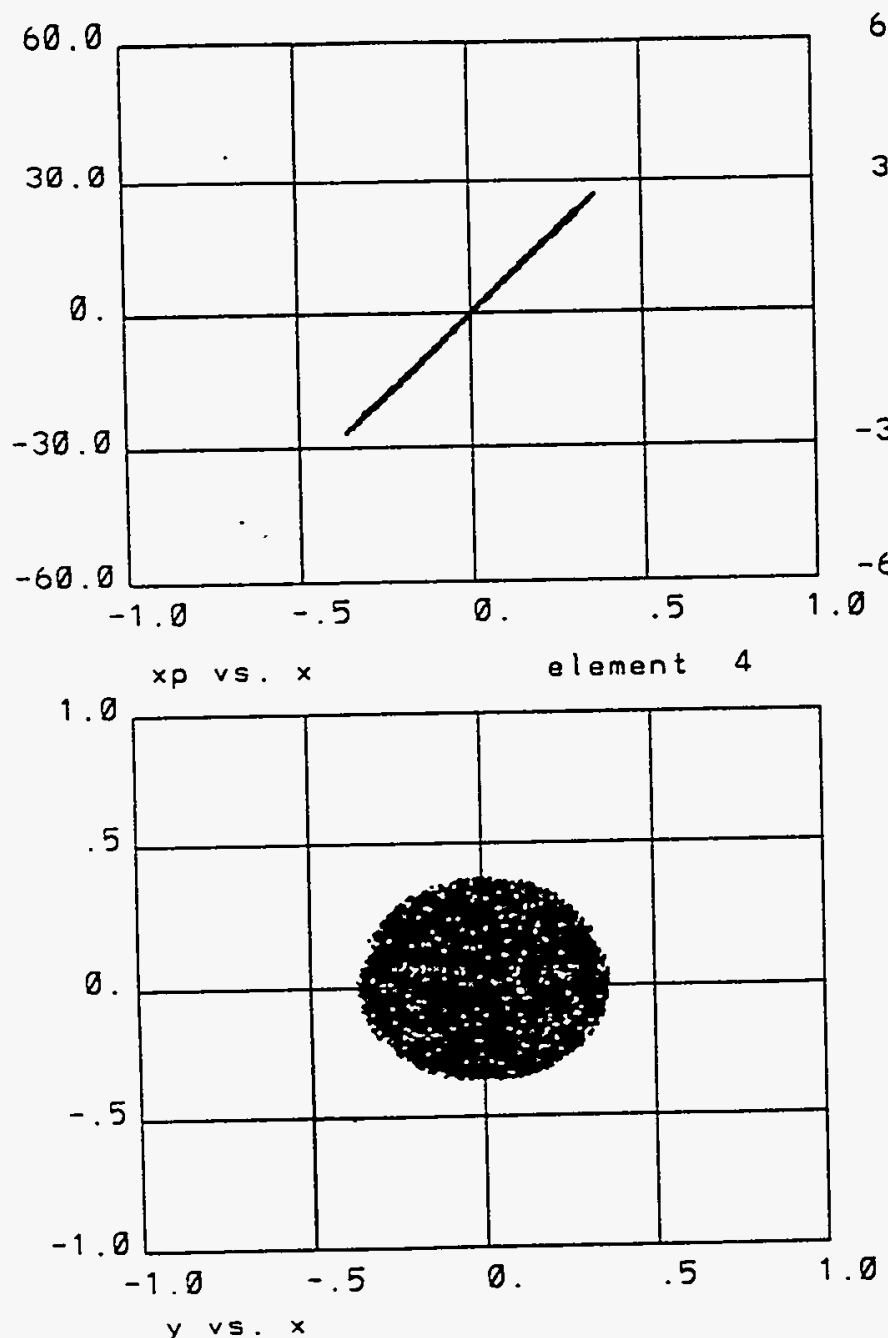

$.5 .15 p s \mathrm{G}(10), 1$ nC, phi $=43.)^{\text {. }}$
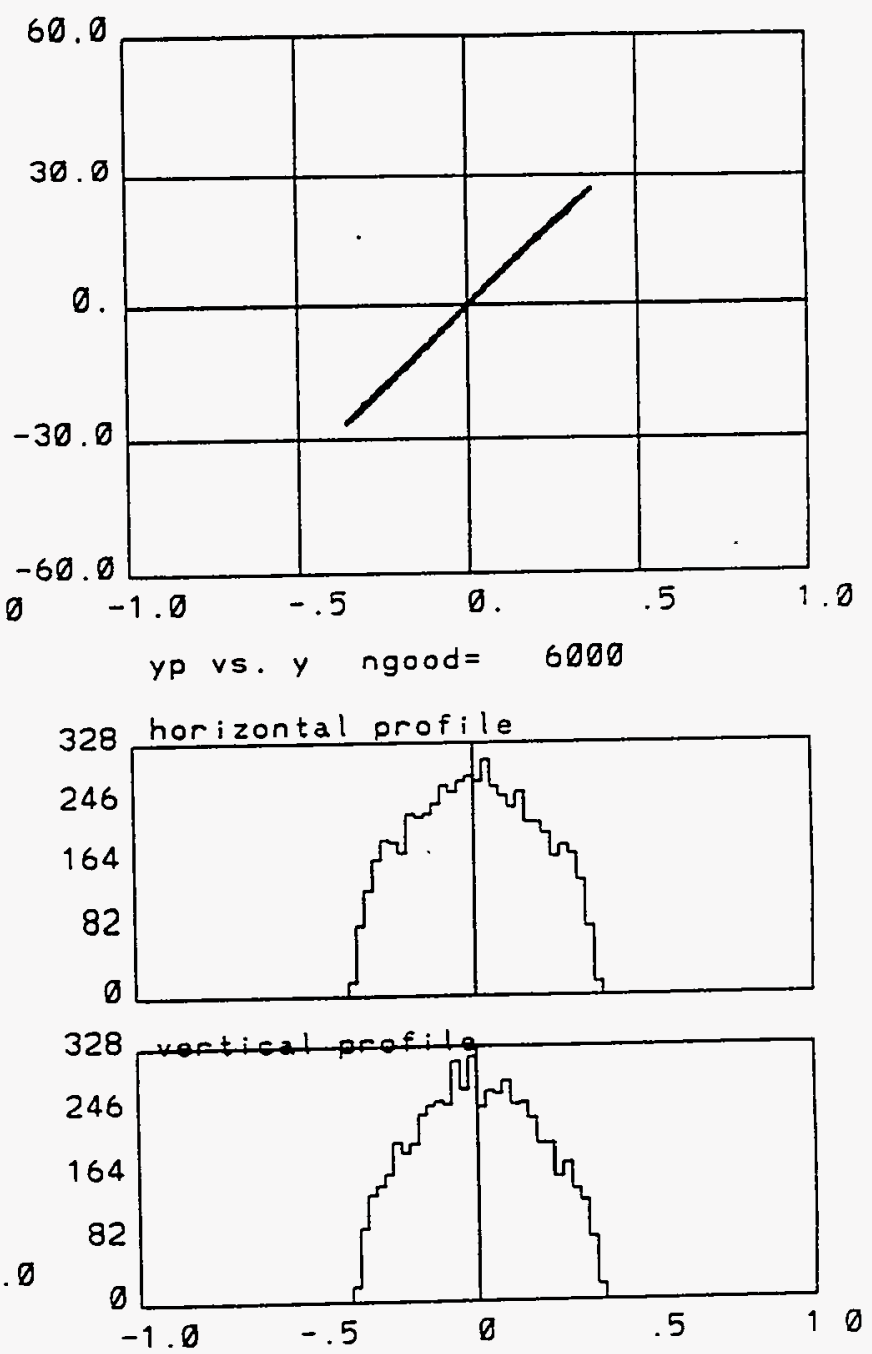
Figure $6-5$ shows the beam profile at position $(n e=5) \quad z=10$, with the Inline-Injection system of Solenoid + Gun + Solenoid combination inline with the linac. With Solenoid current of $I=2200 \mathrm{amp}$.
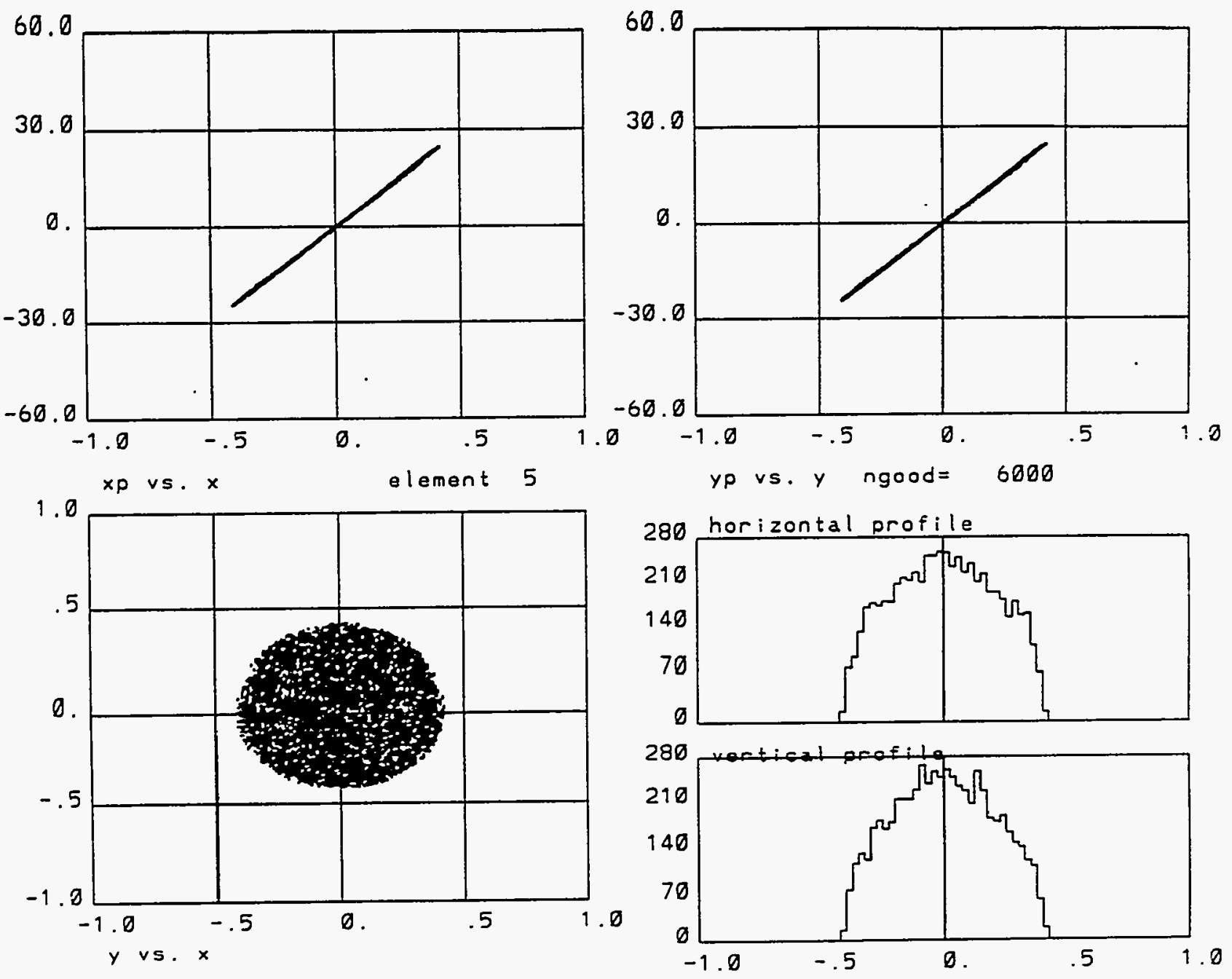
Figure $6-6$ shows the beam profile at position $(n e=10) \quad z=20$, with the Inline-Injection system of Solenoid + Gun + Solenoid combination inline with the linac. With Solenoid current of $I=2200$ amp.

- BNL RF GUN
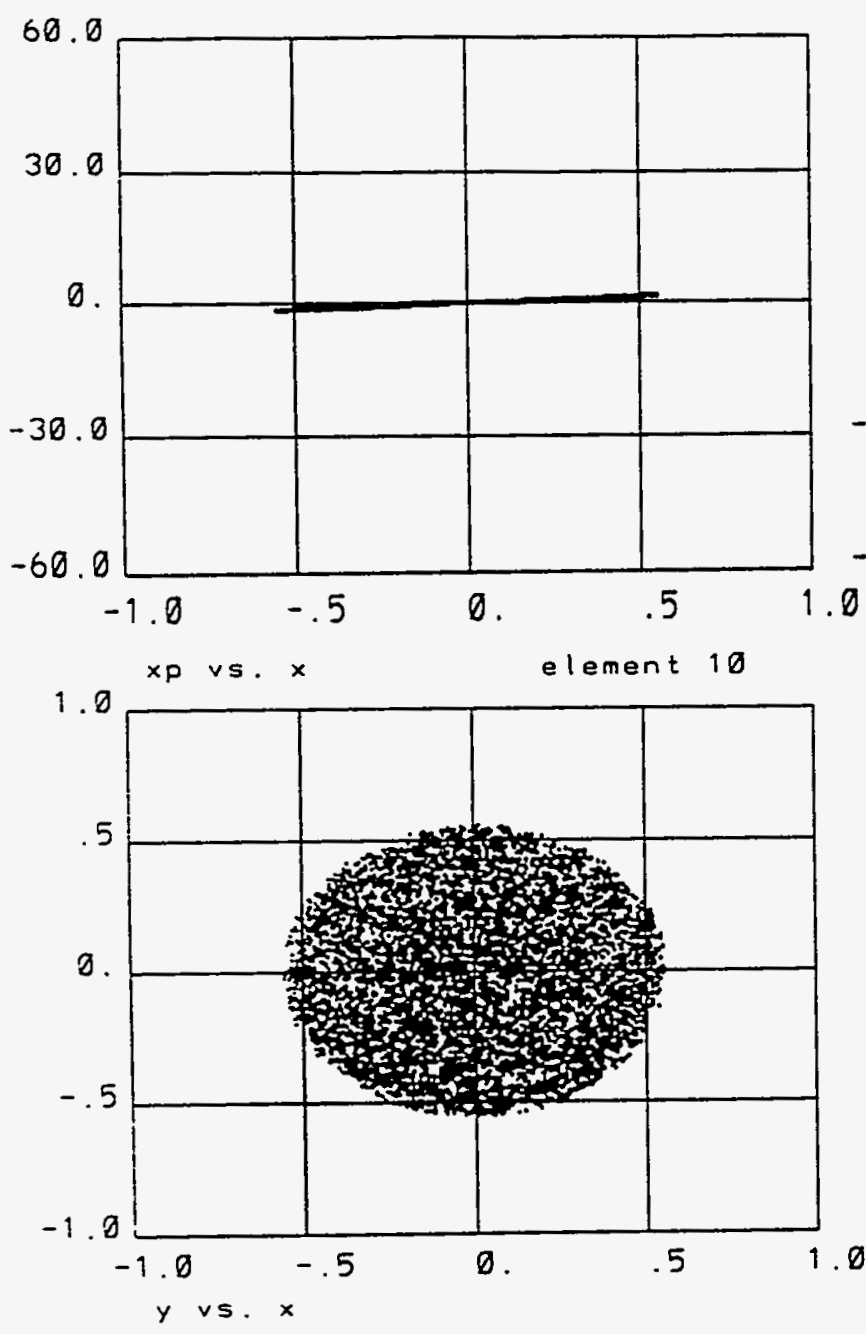

$.5 .150 s G(10), 1$ nC, phi $=43.1$.

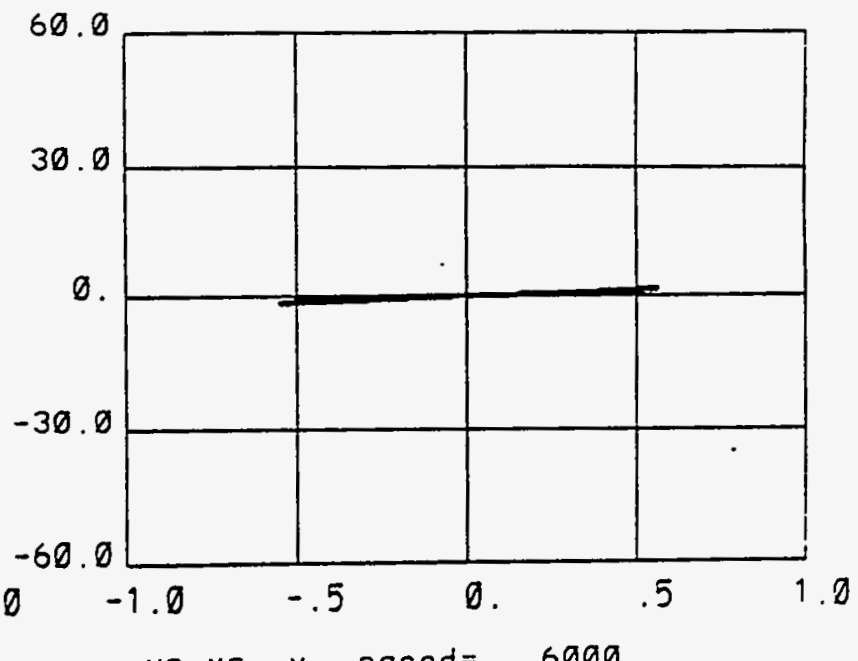

yp vs. y ngood $=6000$
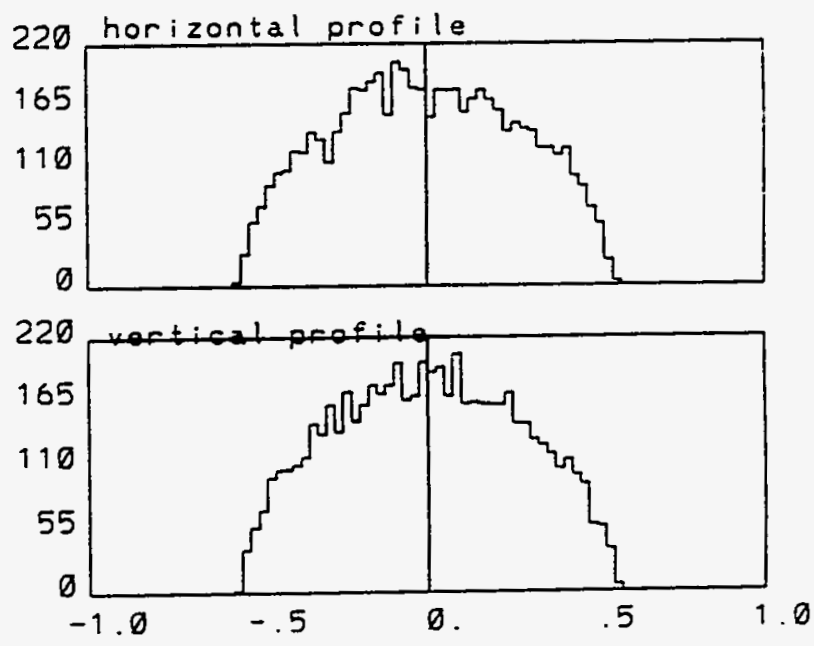
Figure $6-7$ shows the beam profile at position $(n e=20) z=40$, with the Inline-Injection system of Solenoid + Gun + Solenoid combination inline with the linac. With Solenoid current of $\mathrm{I}=2200 \mathrm{amp}$.

- BNL RF GUN

60.0

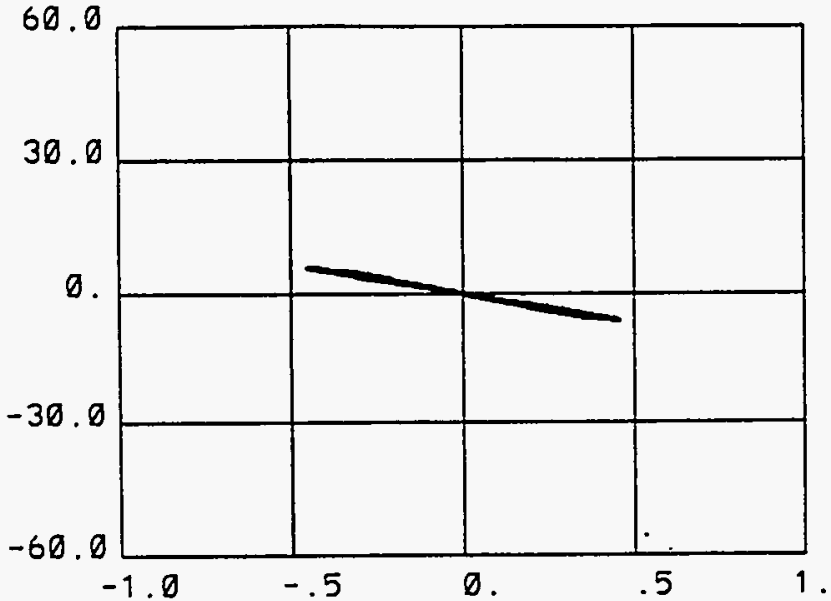

$x p$ vs. $x$ element 20

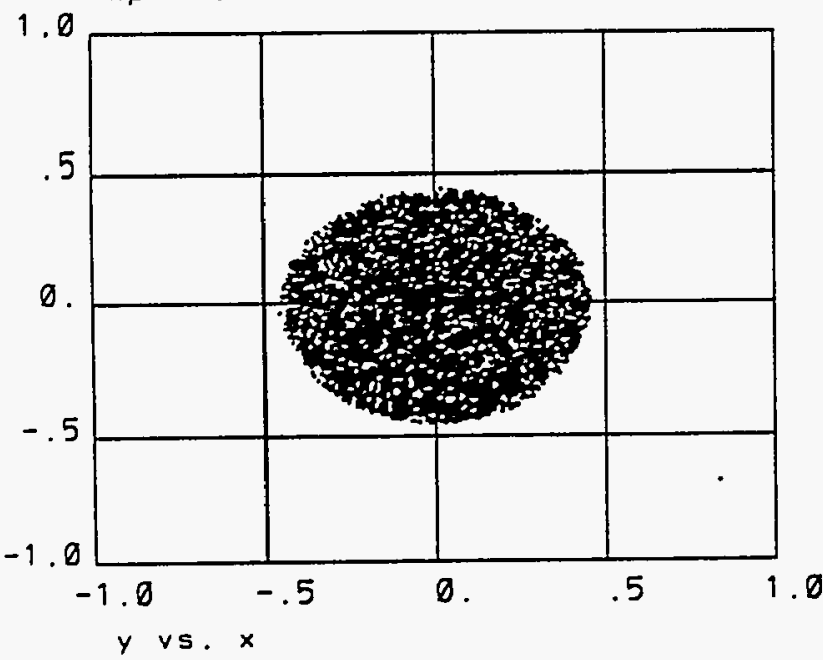

$5.15 p s G(10), 1 n C, p h i=43.1^{\circ}$

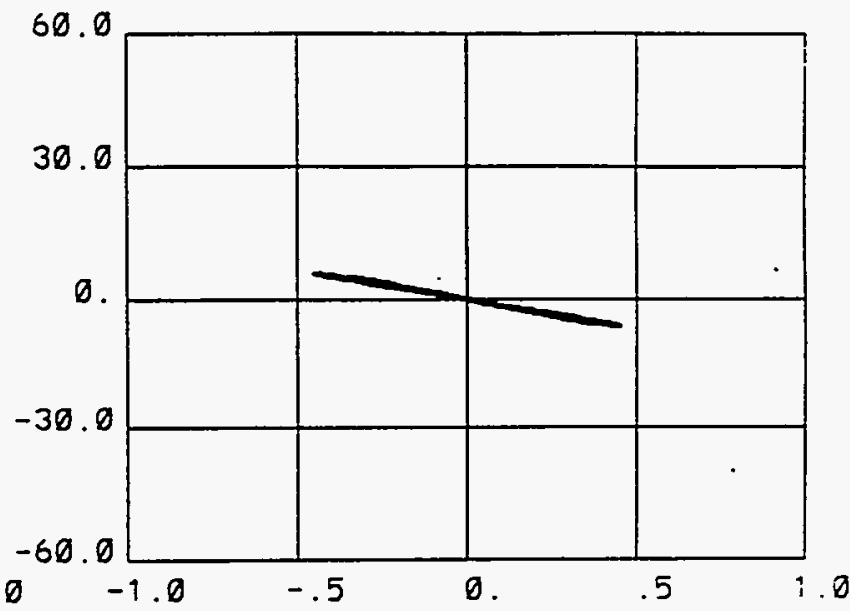

yp vs. y ngood $=6000$
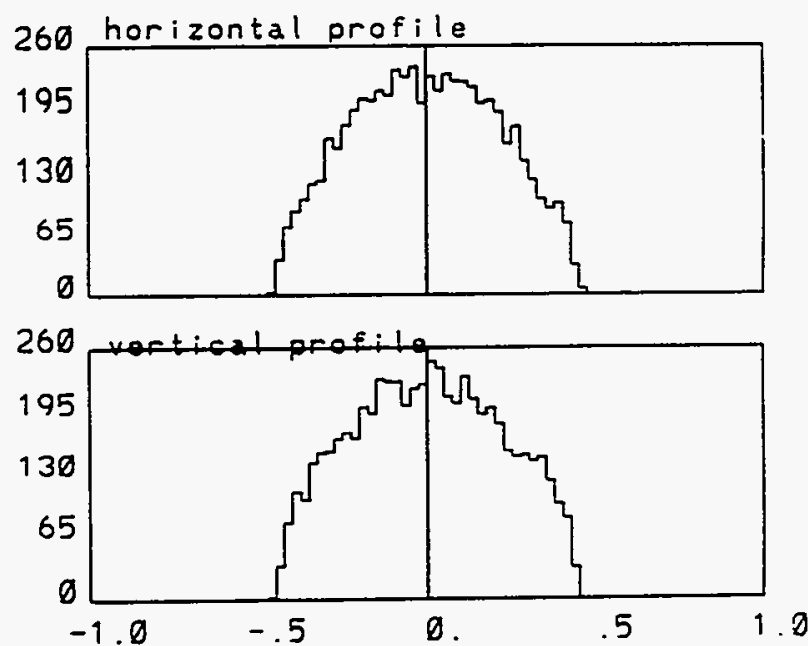
Figure $6-8$ shows the beam profile at position $(n e=30) z=70$, with the Inline-Injection system of Solenoid + Gun + Solenoid combination inline with the linac. With Solenoid current of $I=2200$ amp.

-BNL RF GUN

50.

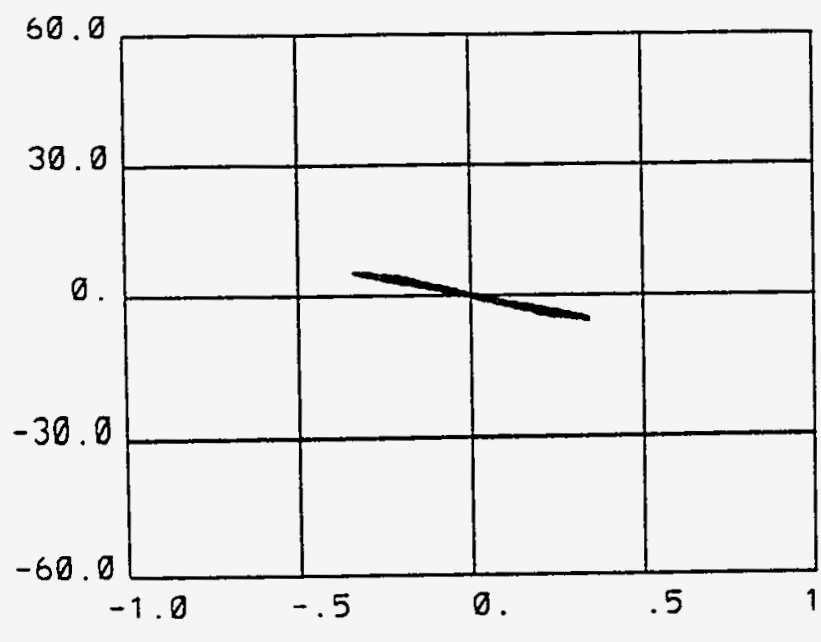

$x$ vs. $x$ element 30

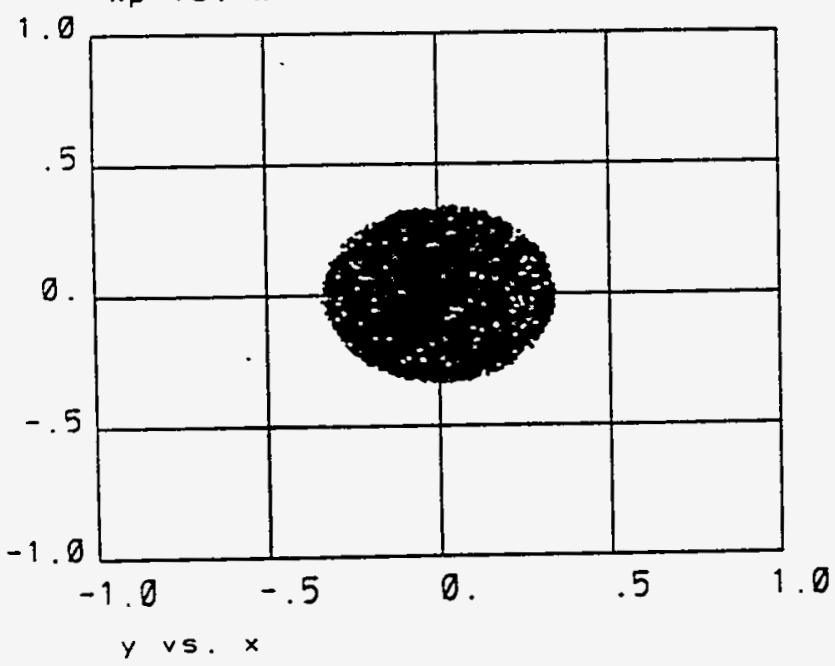

$, 5.15 p s G(10), 1 n C$, phi $=43.)^{\circ}$

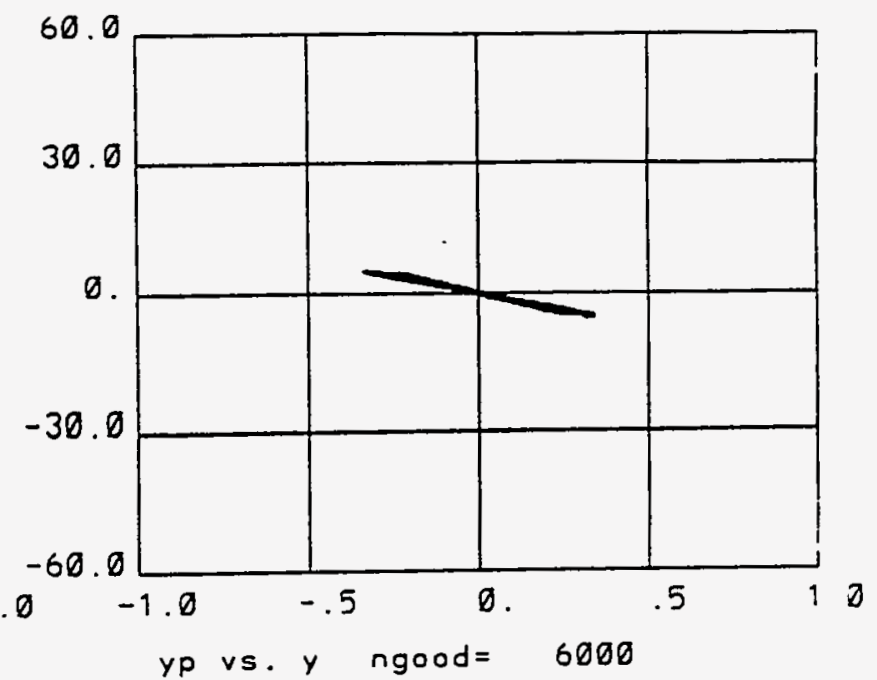

yp vs. y ngood= 6000
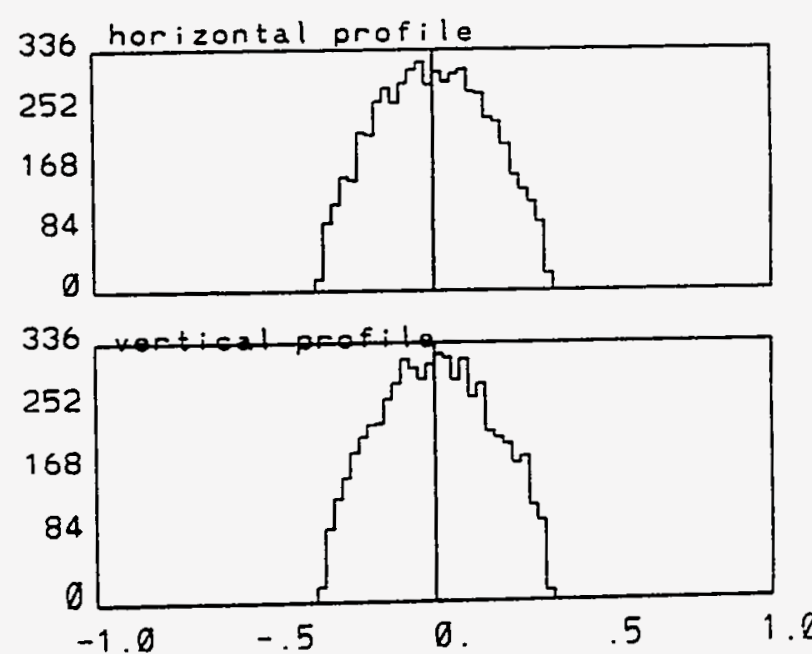
Figures $7-1$ to $7-8$ shows the beam profile at various positions (distance $z$ from the cathode), with the Inline-Injection system of Solenoid + Gun + Solenoid combination inline with the linac. With Solenoid current of I=2400 amp.

- BNL RF GUN
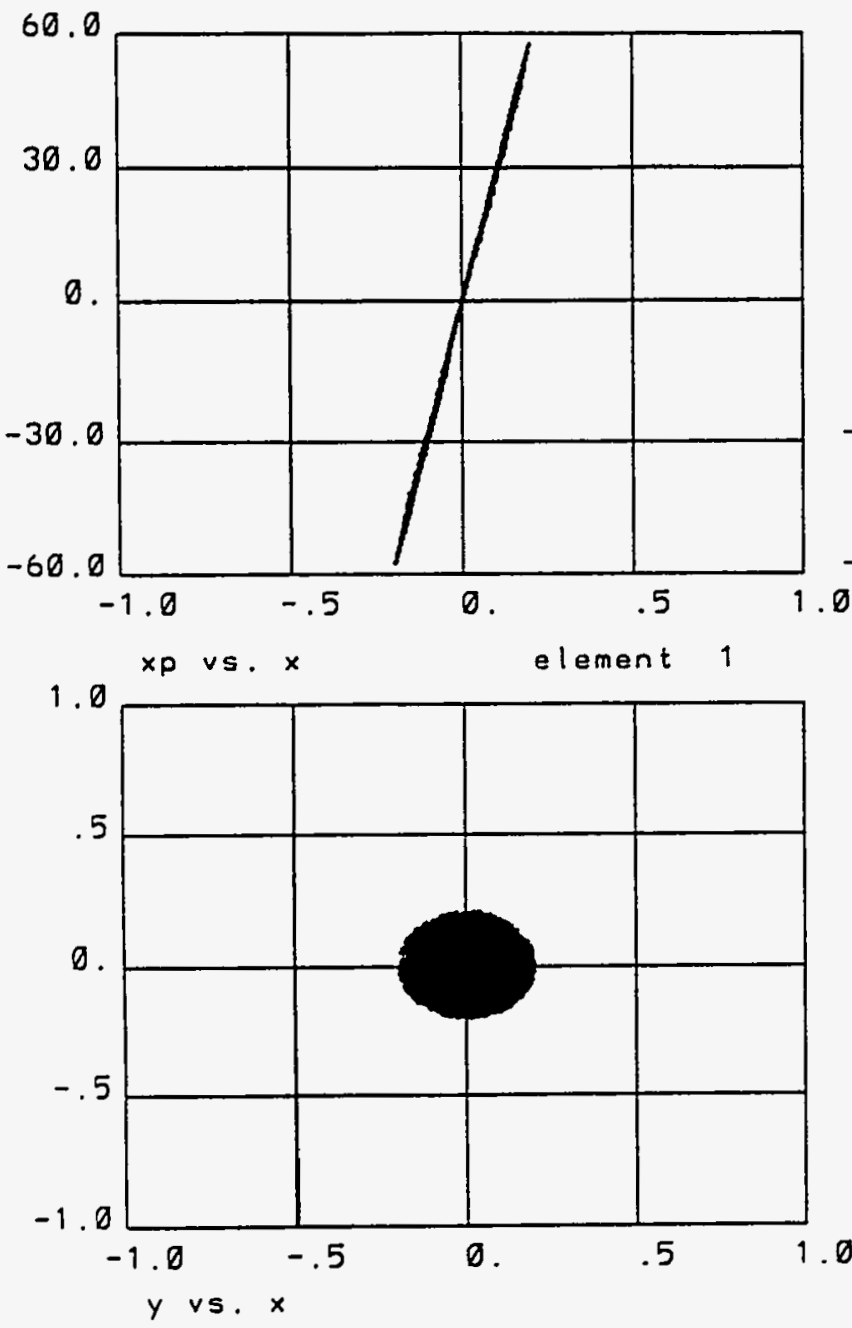

$15.15 p s \mathrm{G}(10), 1 \mathrm{nC}, \mathrm{phi}=43.1$
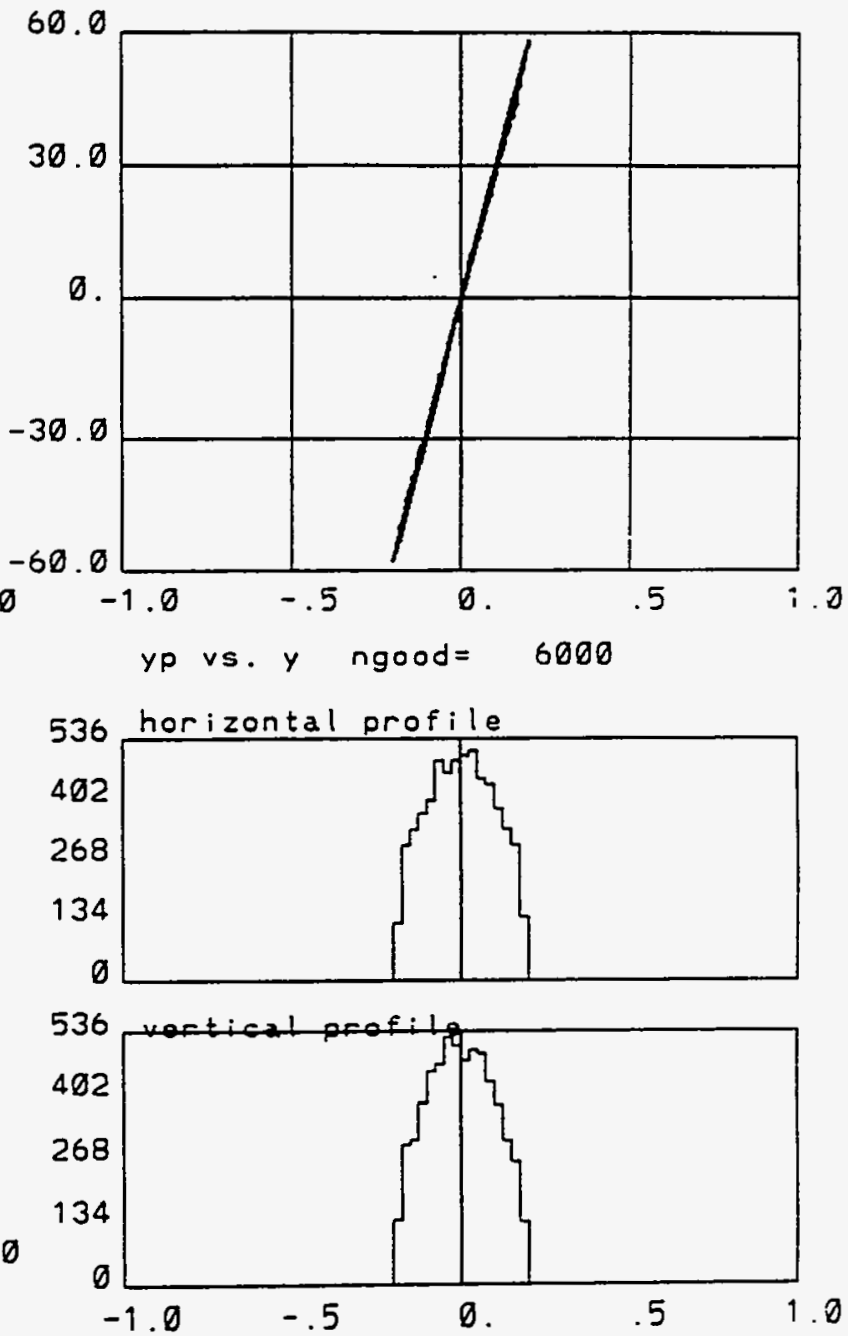

Figure $7-1$ shows the beam profile at position $(n e=1) z=0$, with the Inline-Injection system of Solenoid + Gun + Solenoid combination inline with the linac. With Solenoid current of $I=2400 \mathrm{amp}$. 
Figure 7-2 shows the beam profile at position $(n e=2) z=2.6$, with the Inline-Injection system of Solenoid + Gun + Solenoid combination inline with the linac. With Solenoid current of $I=2400 \mathrm{amp}$.

- BNL RF GUN

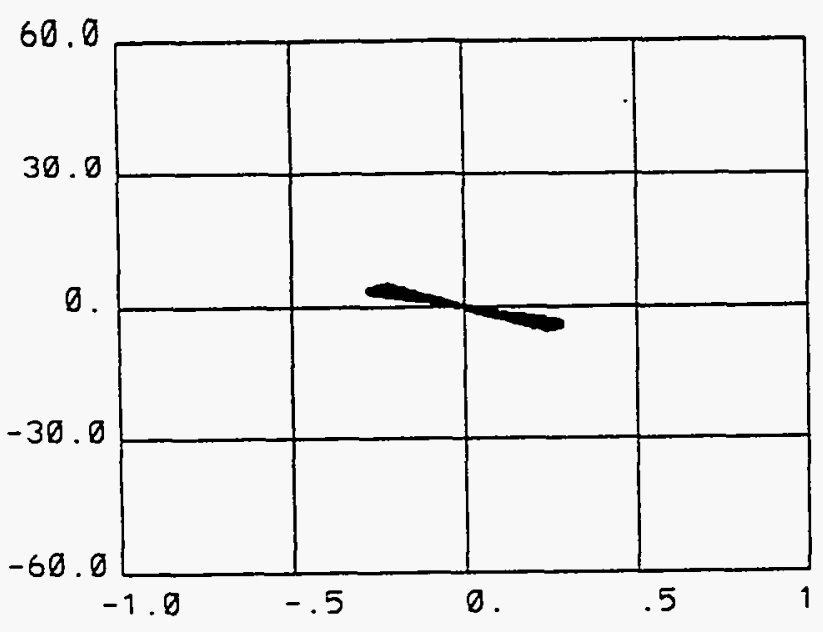

$x p v s . x$ element 2

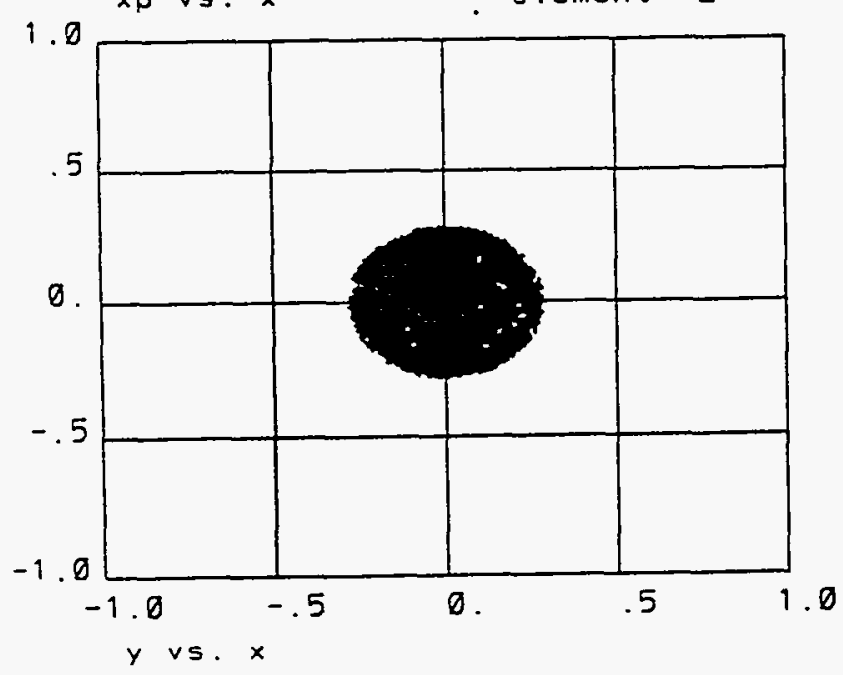

$5.15 p s$ G $(10), 1 n C, p h i=43.1$.

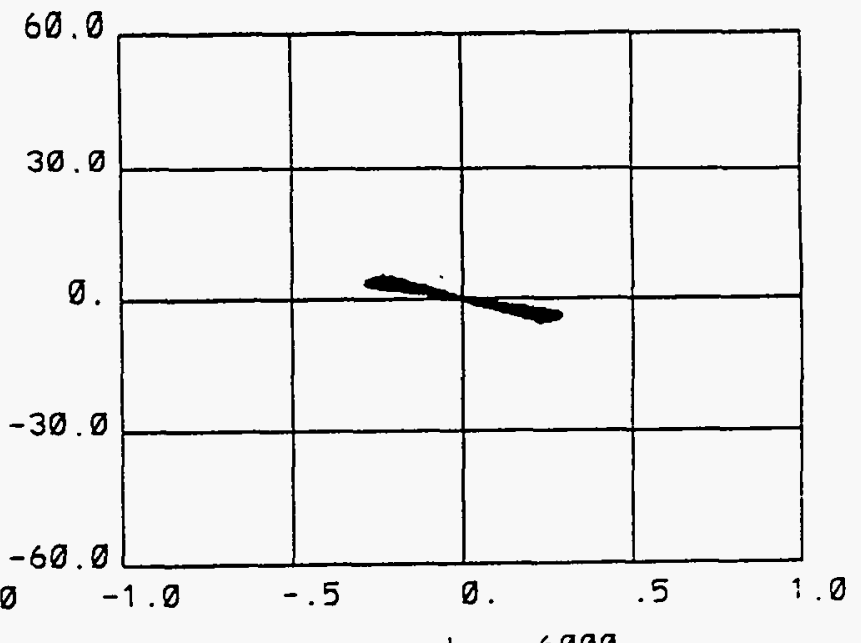

yp vs. y ngood $=6000$
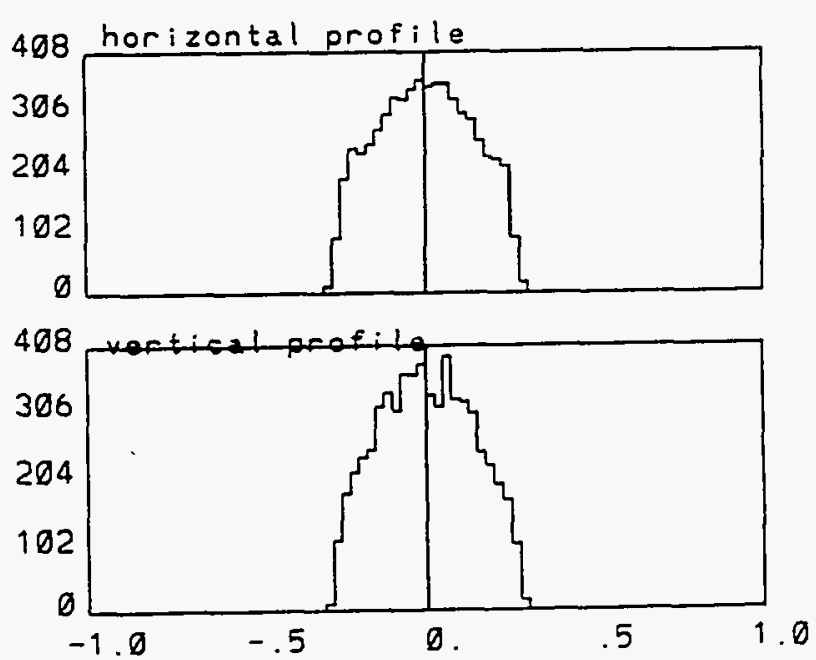
Figure 7-3 shows the beam profile at position $(n e=3) z=5.2$, with the Inline-Injection system of Solenoid + Gun + Solenoid combination inline with the linac. With Solenoid current of $I=2400$ amp

- BNL RF GUN IF
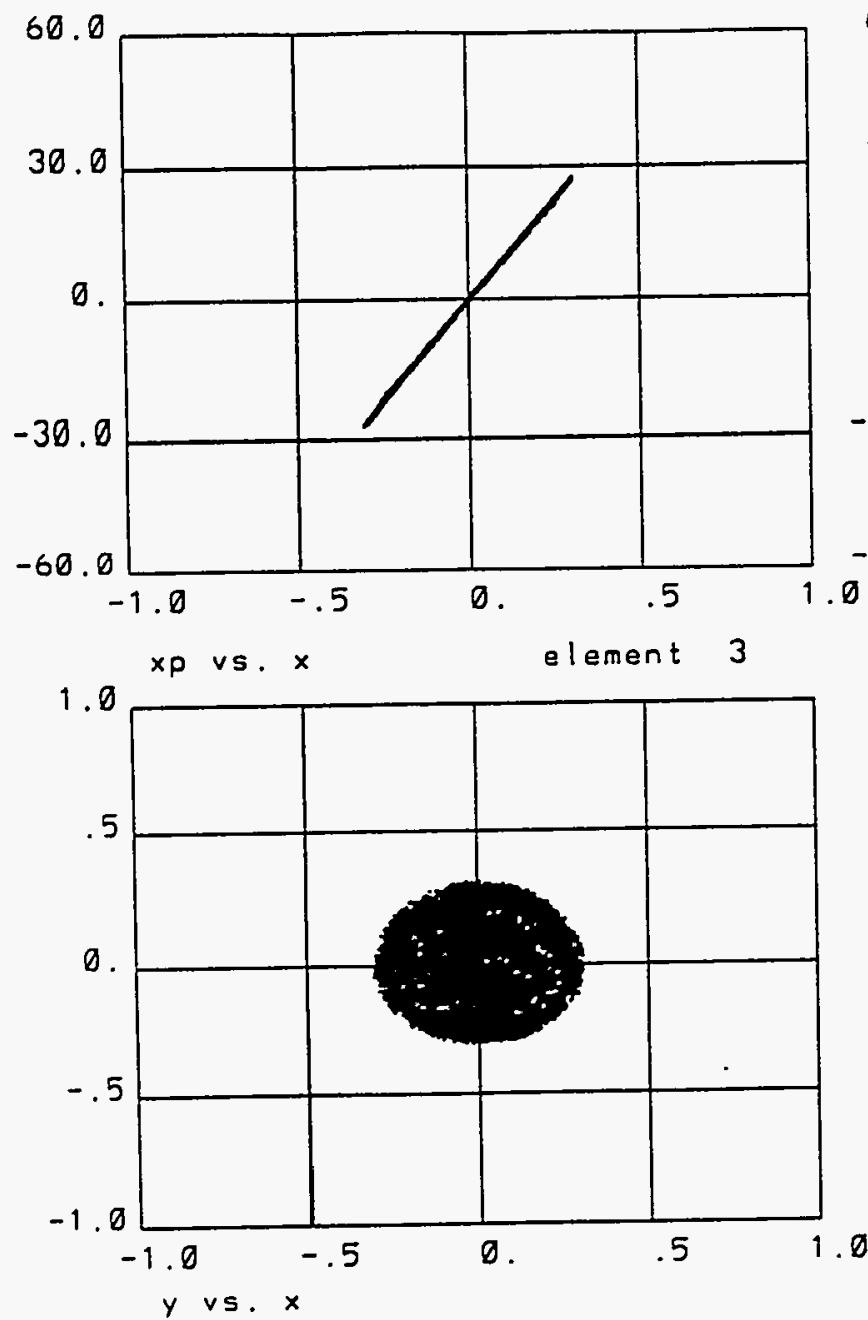

$.5 .15 p s G(10), 1 n C$, ohi $=43.)^{\circ}$
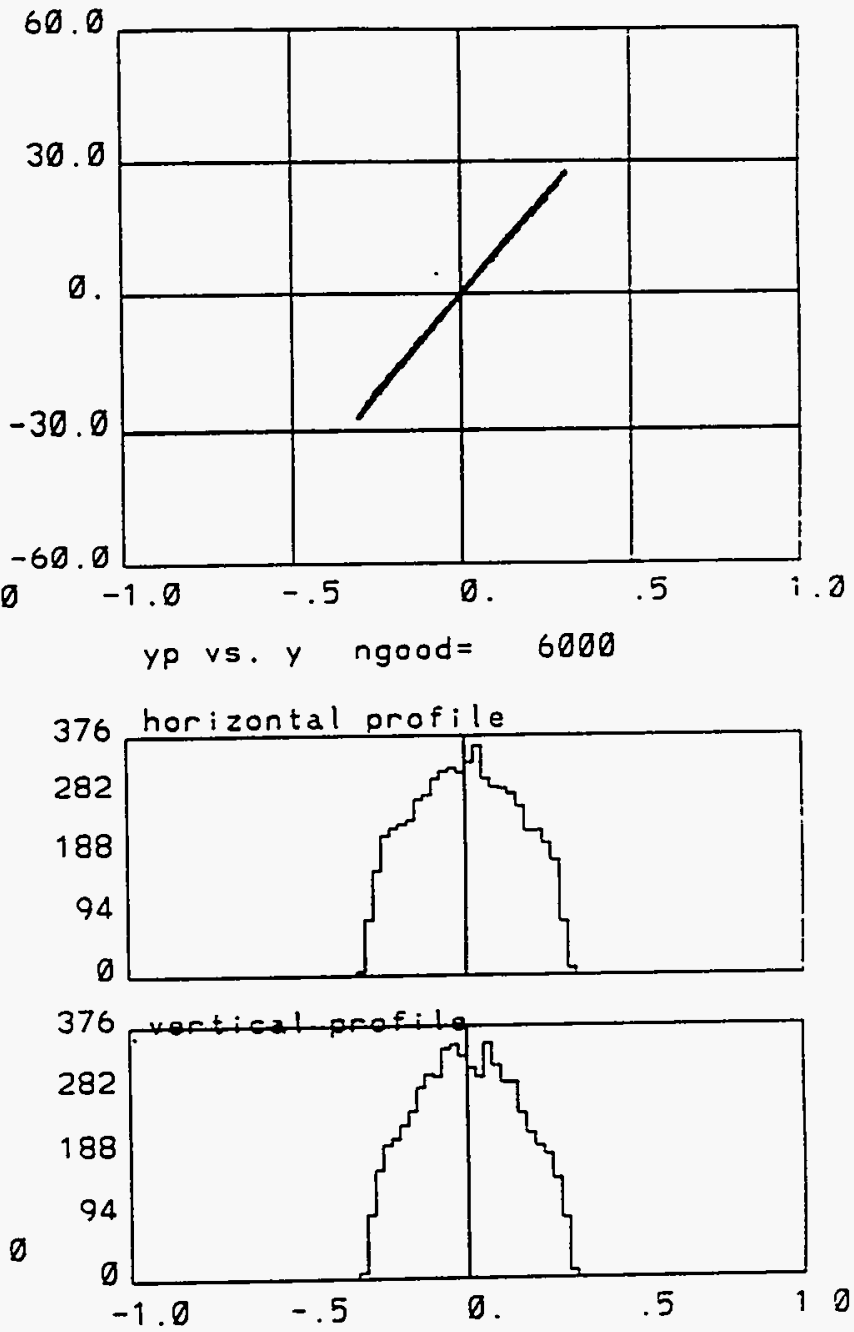
Figure $7-4$ shows the beam profile at position $(n e=4) z=7.9$, with the Inline-Injection system of Solenoid + Gun + Solenoid combination inline with the linac. With Solenoid current of $I=2400$ amp.

- BNL RF GUN
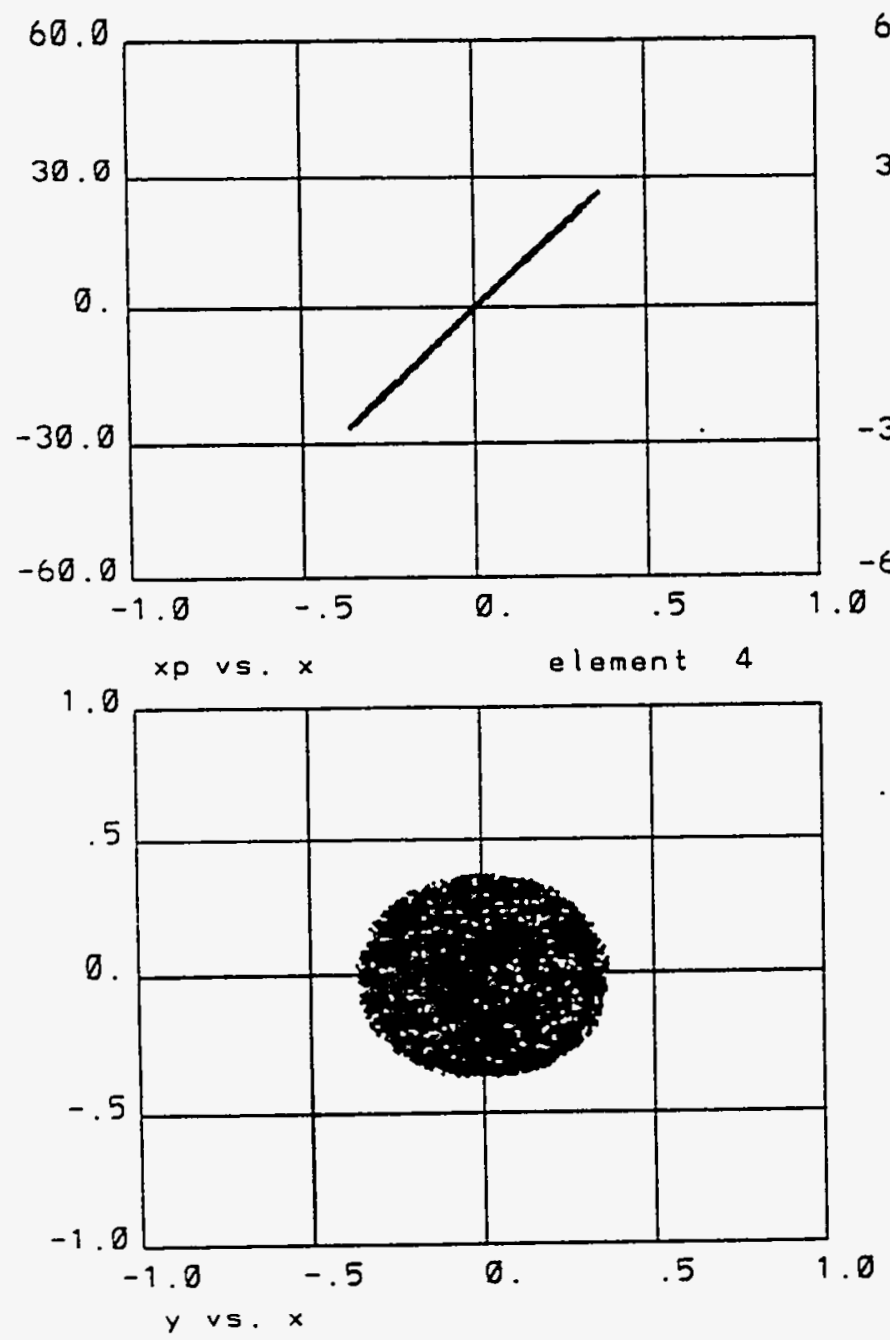

15.15ps $G(10), 1 n C$, phi $=43.)^{\prime}$
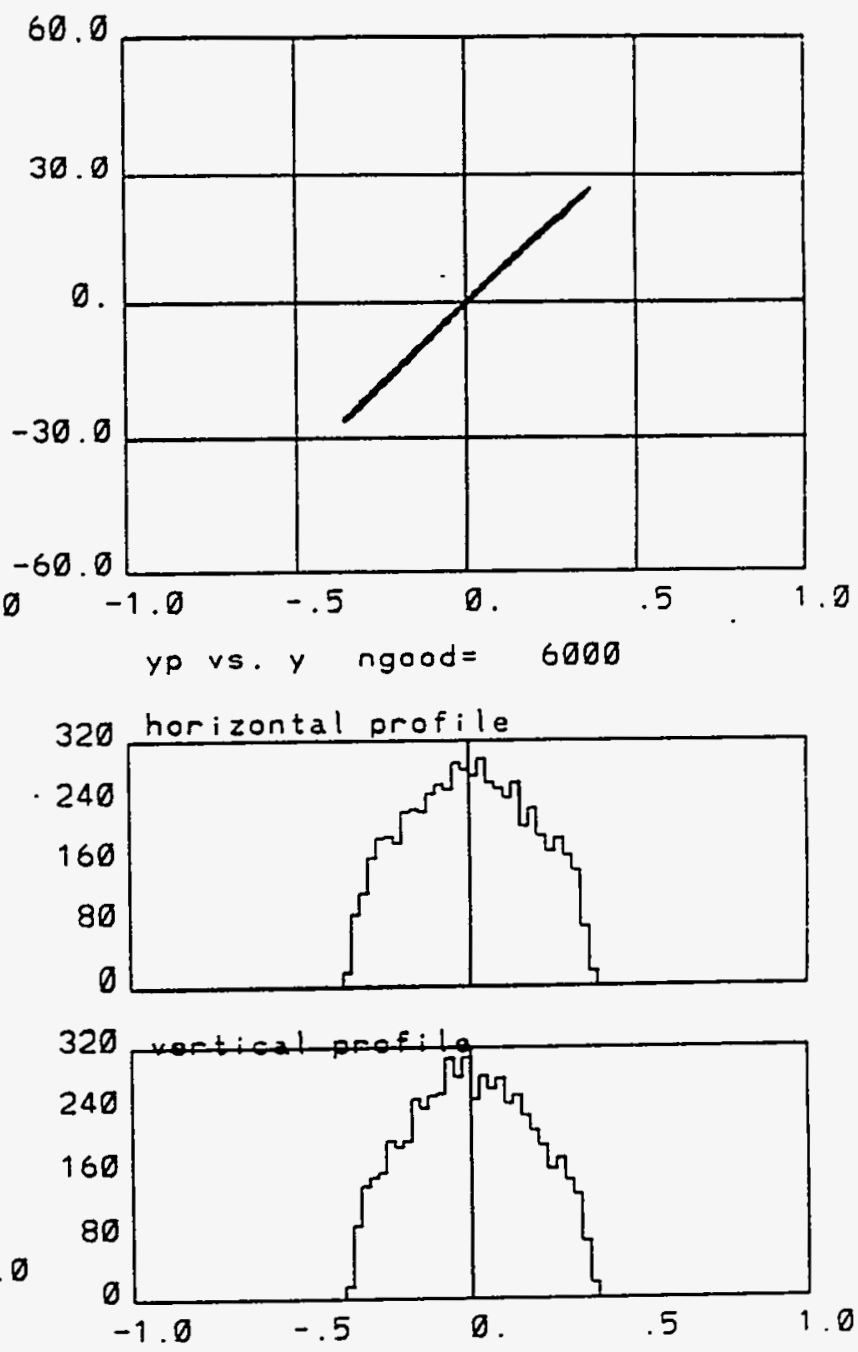
Figure 7 - 5 shows the beam profile at position $(n e=5) z=10$, with the Inline-Injection system of Solenoid + Gun + Solenoid combination inline with the linac. With Solenoid current of $I=2400 \mathrm{amp}$.

-BNL RF GUN
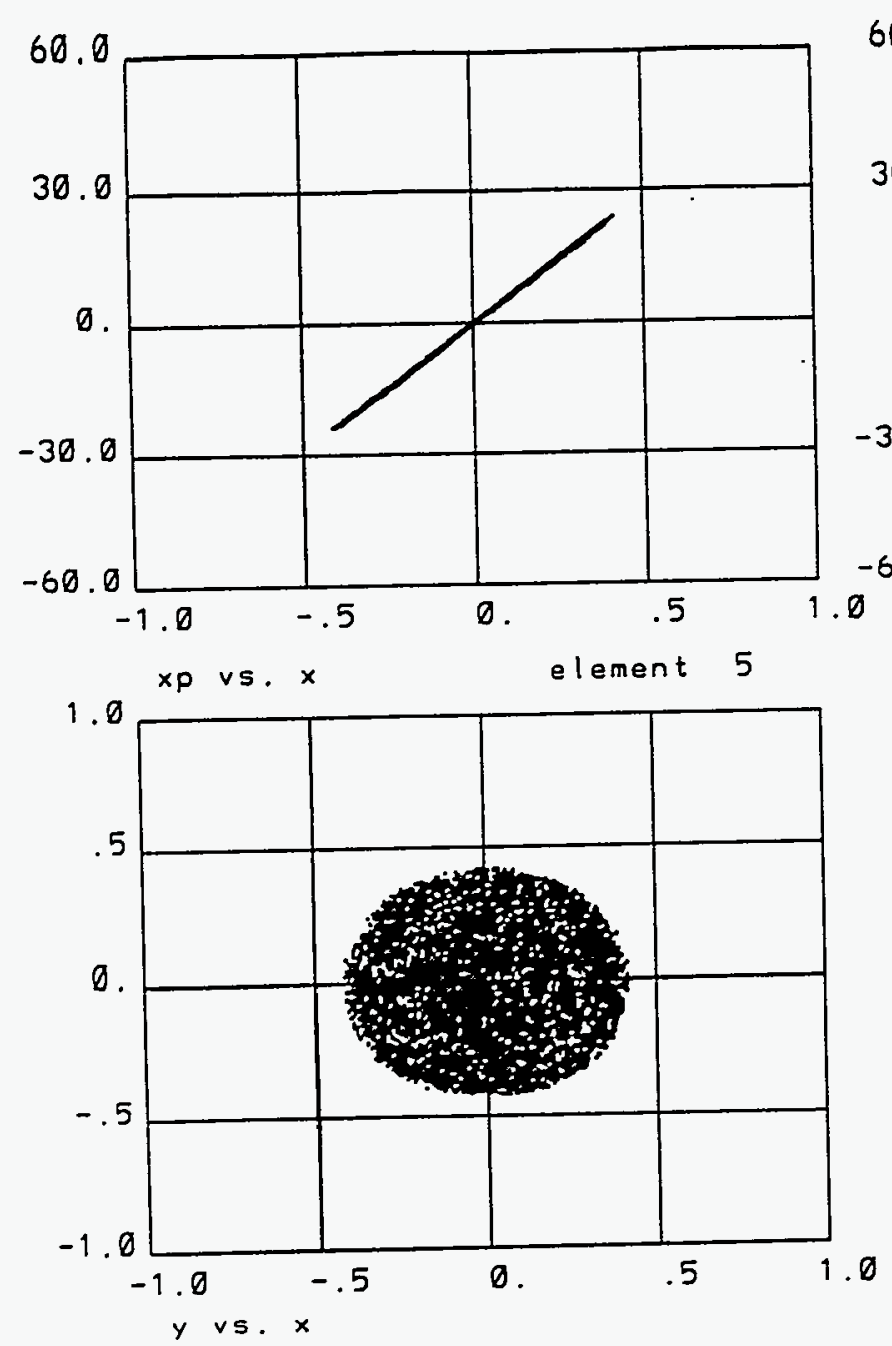

15.15ps $G(10), 1 \mathrm{nC}, \mathrm{phi}=43.1^{\circ}$
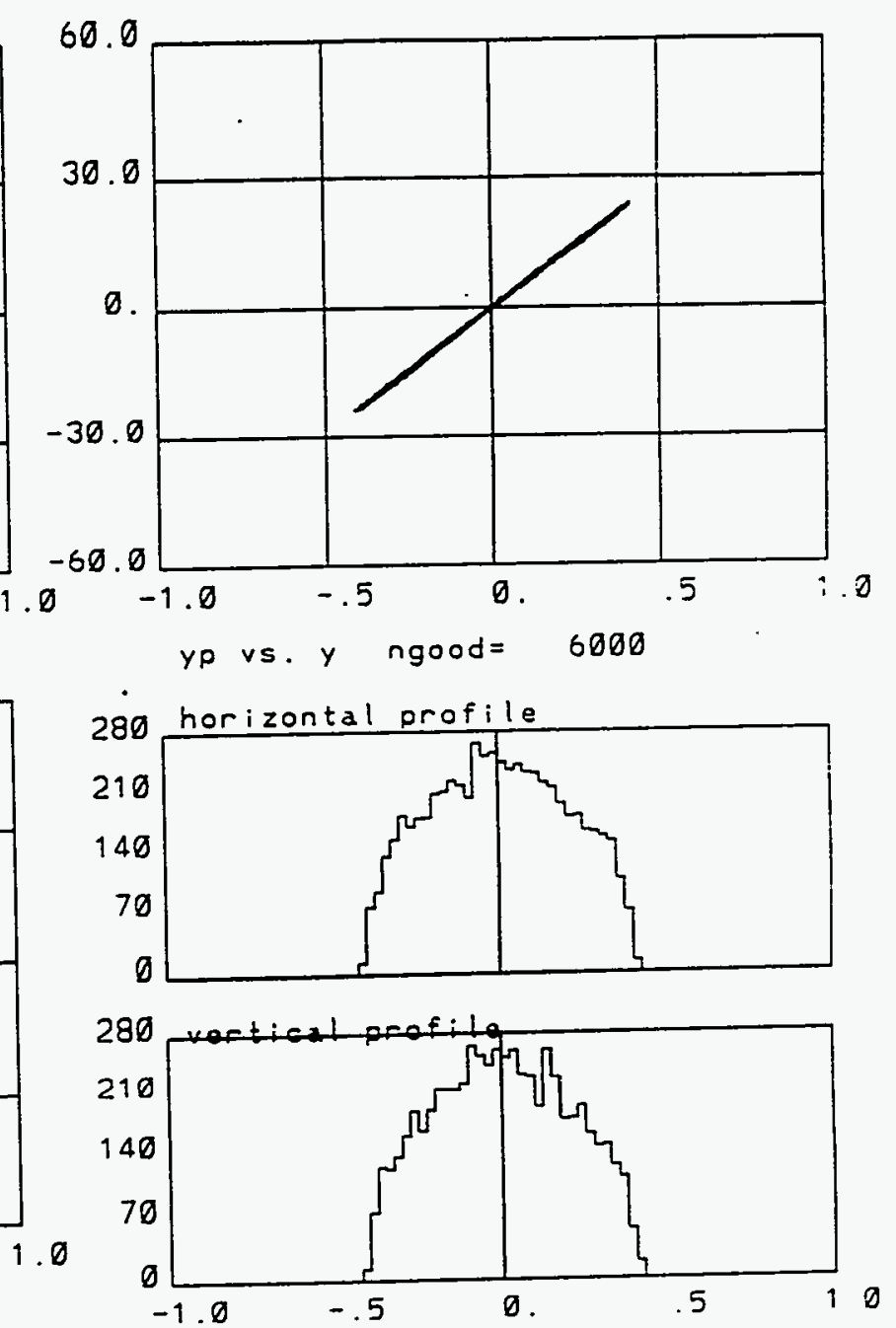
Figure 7-6 shows the beam profile at position $(n e=10) z=20$, with the Inline-Injection system of Solenoid + Gun + Solenoid combination inline with the linac. With Solenoid current of $I=2400$ amp.

- BNL RF 'GUN

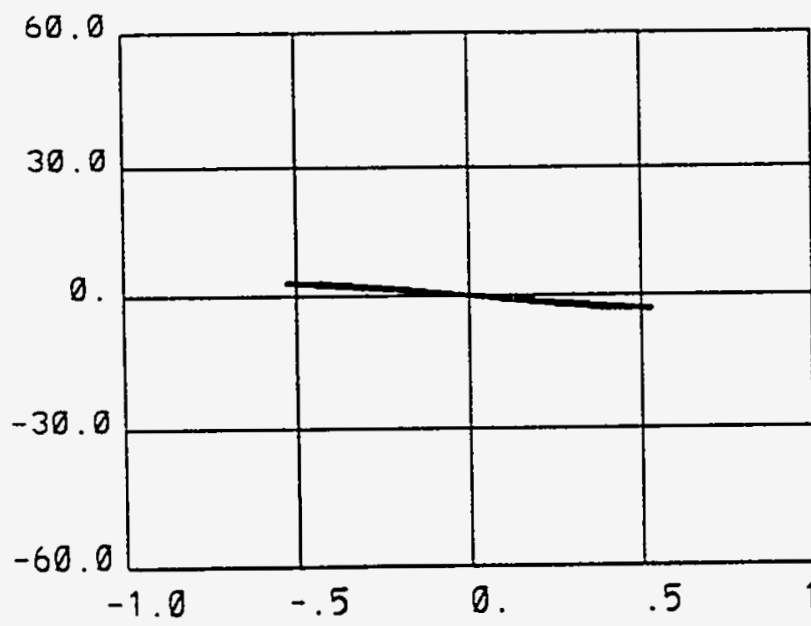

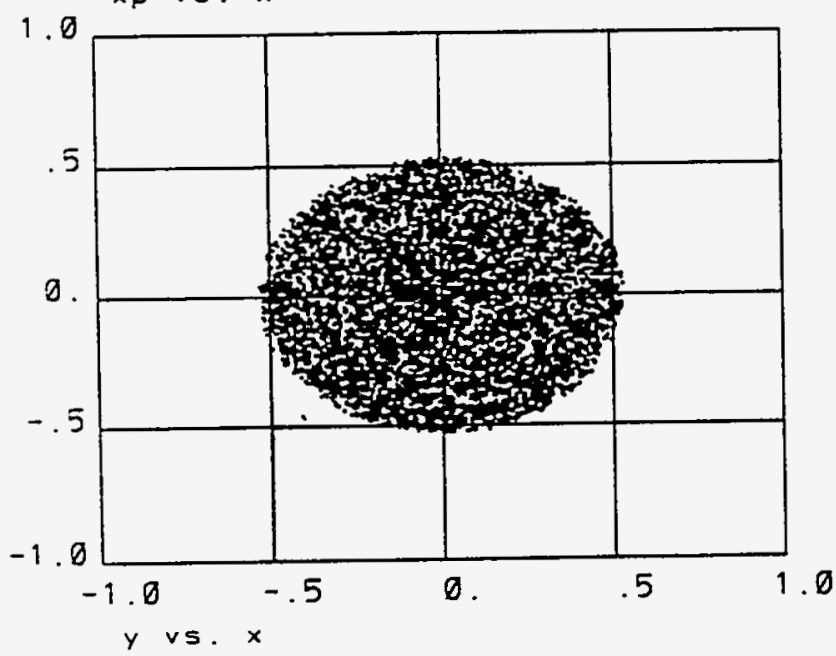

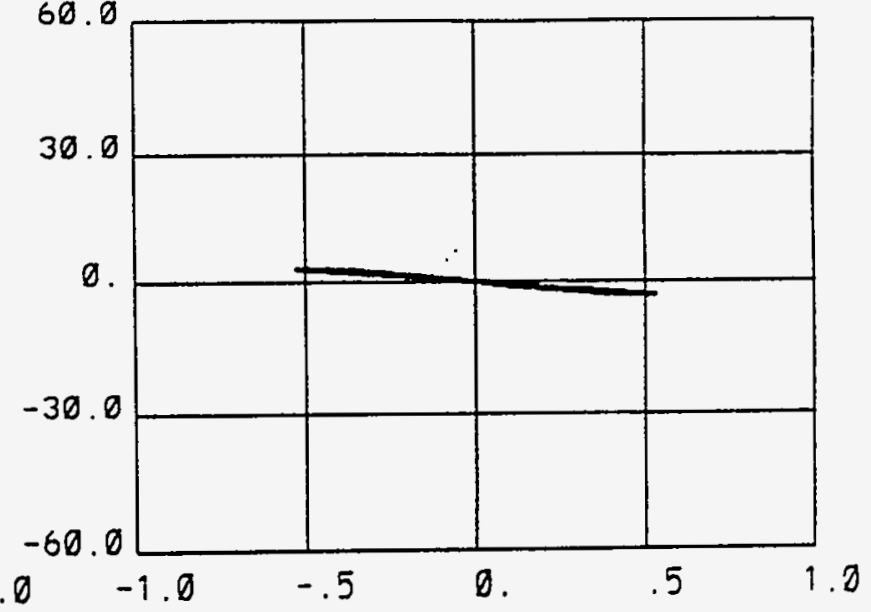

yp vs. y ngood $=6000$
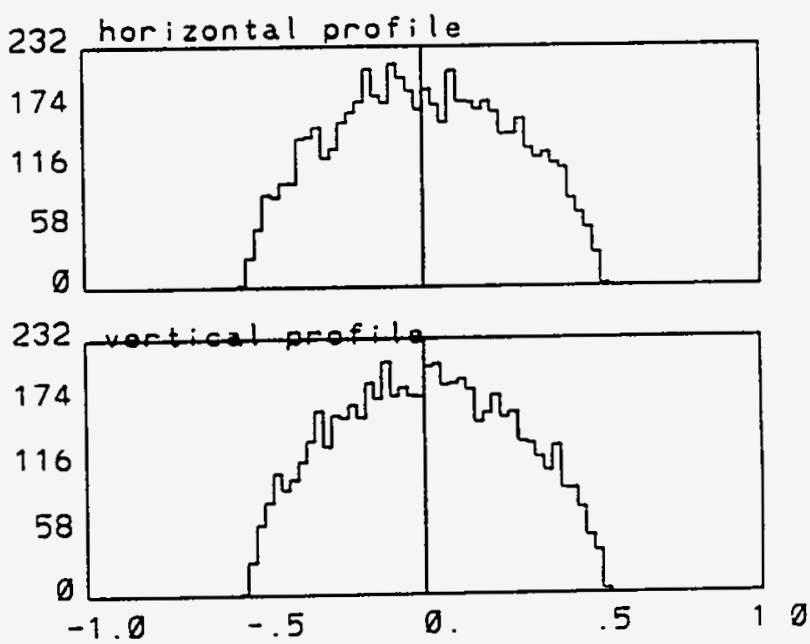
Figure $7-7$ shows the beam profile at position $(n e=20) z=40$, with the Inline-Injection system of Solenoid + Gun + Solenoid combination inline with the linac. With Solenoid current of $I=2180 \mathrm{amp}$.

- BNL RF GUN
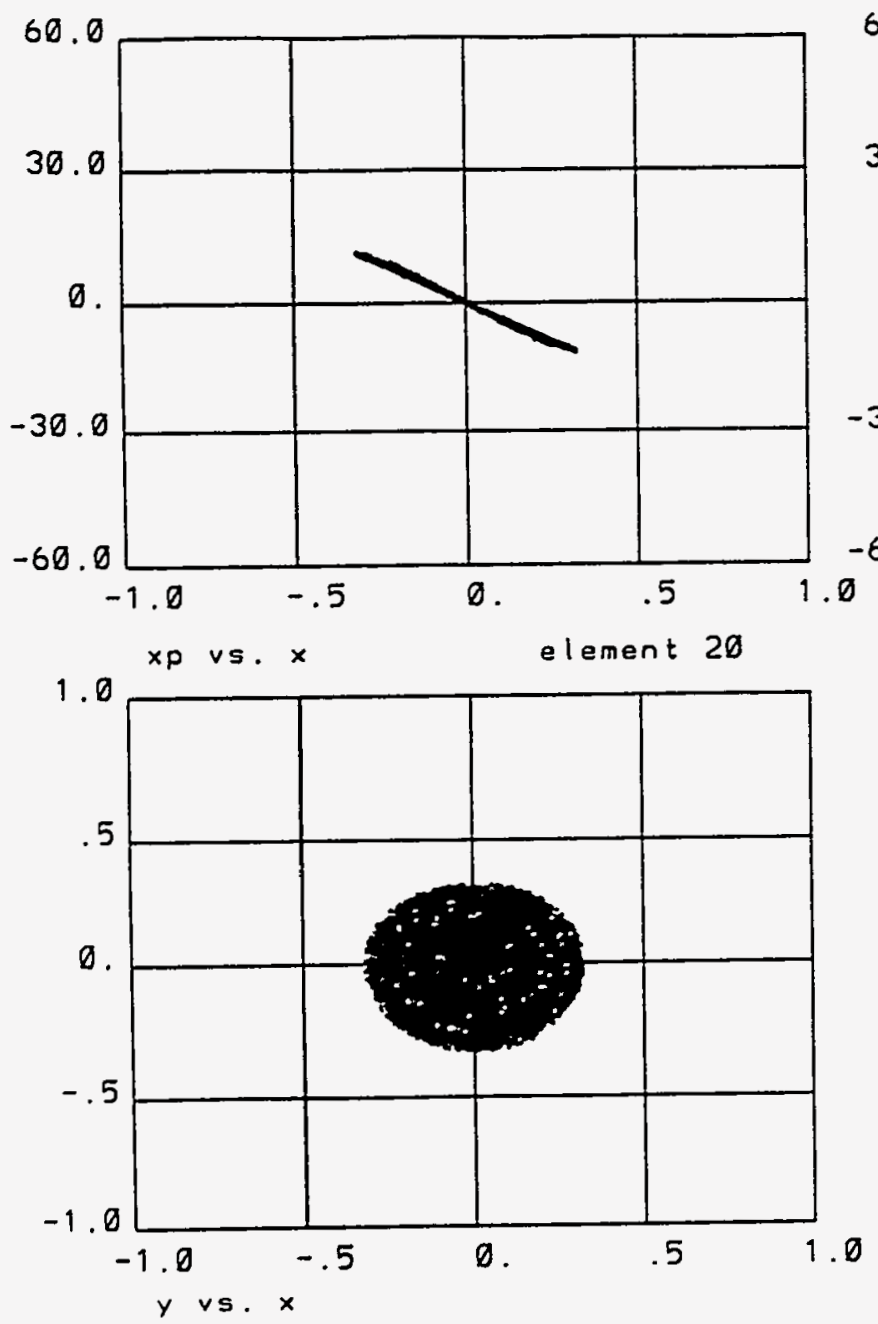

$, 5.15 p s G(10), 1 n C$, phi $=43.1^{\circ}$
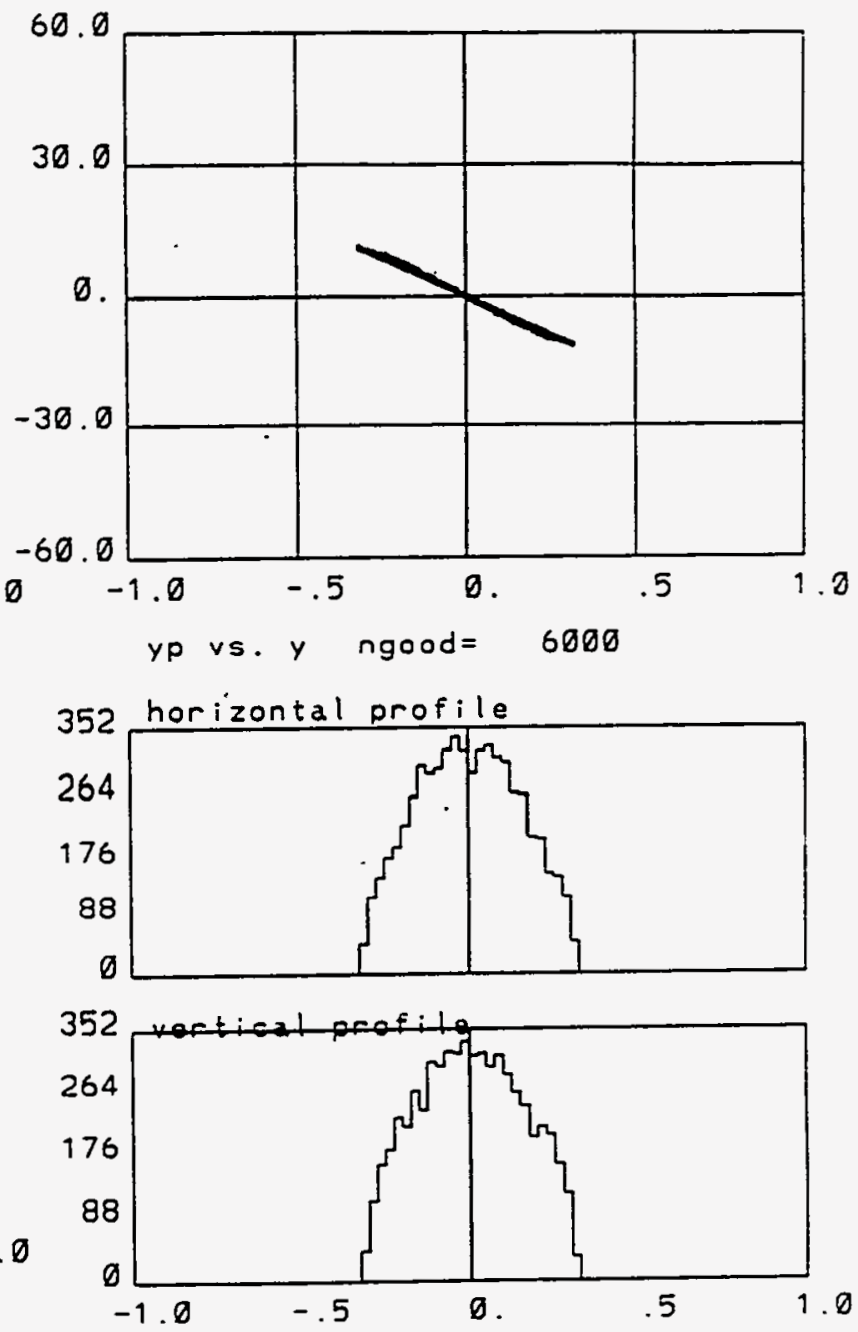
Figure 7-8 shows the beam profile at position $(n e=30) \quad z=70$, with the Inline-Injection system of Solenoid + Gun + Solenoid combination inline with the linac. With Solenoid current of $\mathrm{I}=2400$ amp.

-BNL RF GUN
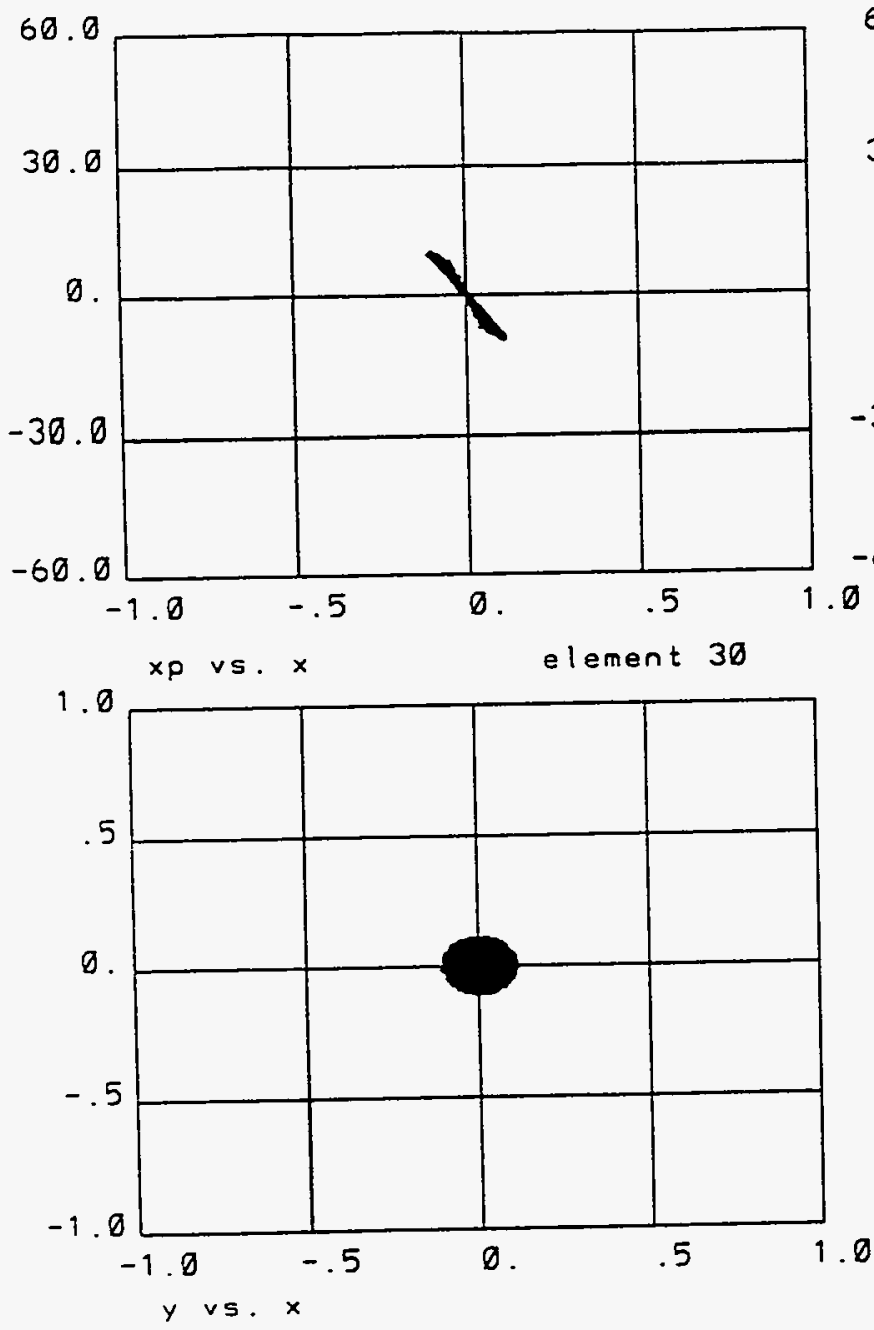

$, 5.15 p s$ G $(10), 1 \mathrm{nC}$, phi $=43.)^{\circ}$
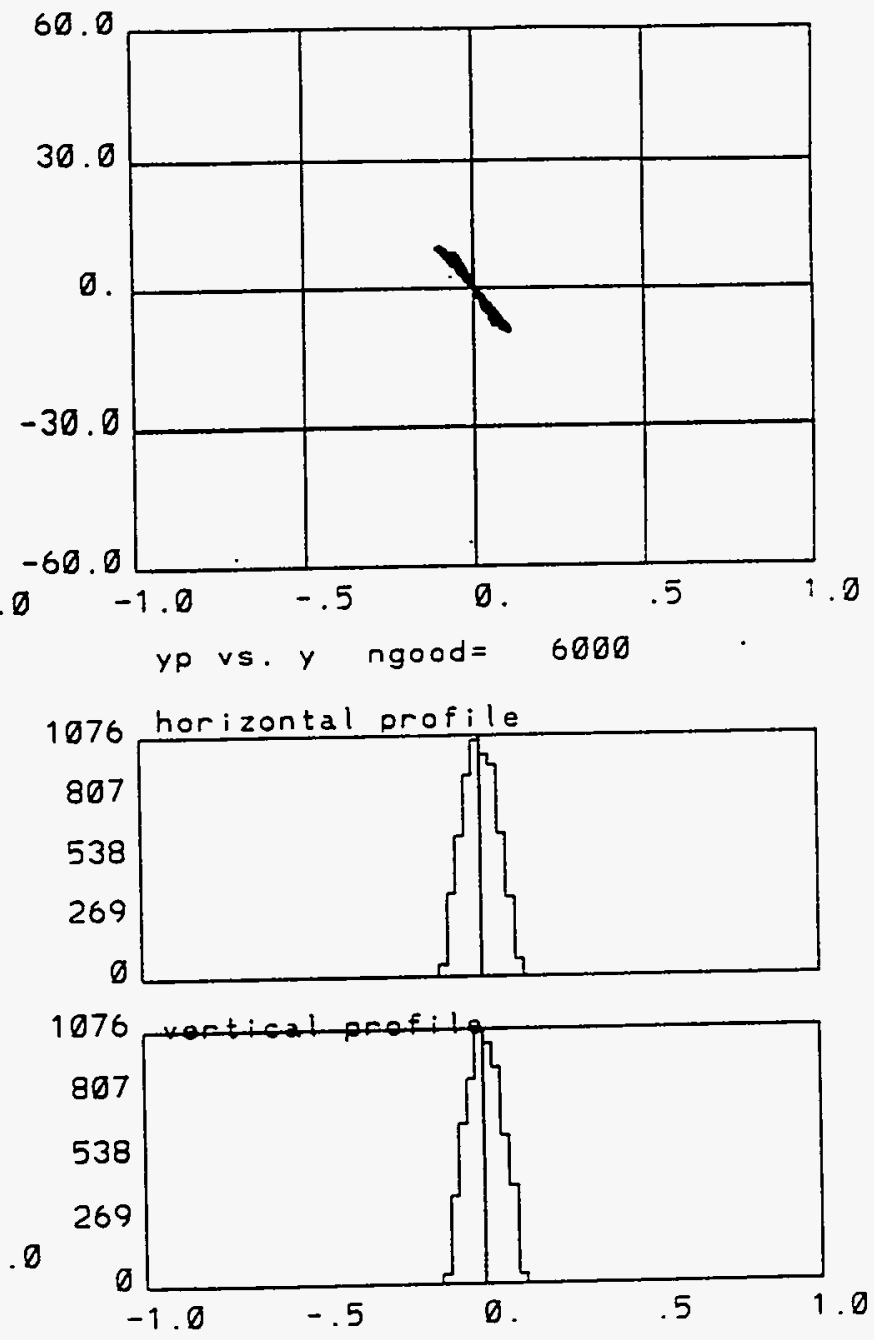
Figures $8-1$ to 8-10 shows the beam profile at various positions (distance $z$ from the cathode), with the Inline-Injection system of Solenoid + Gun + Solenoid combination inline with the linac. With Solenoid current of I=2500 amp.

- BNL RF GUN
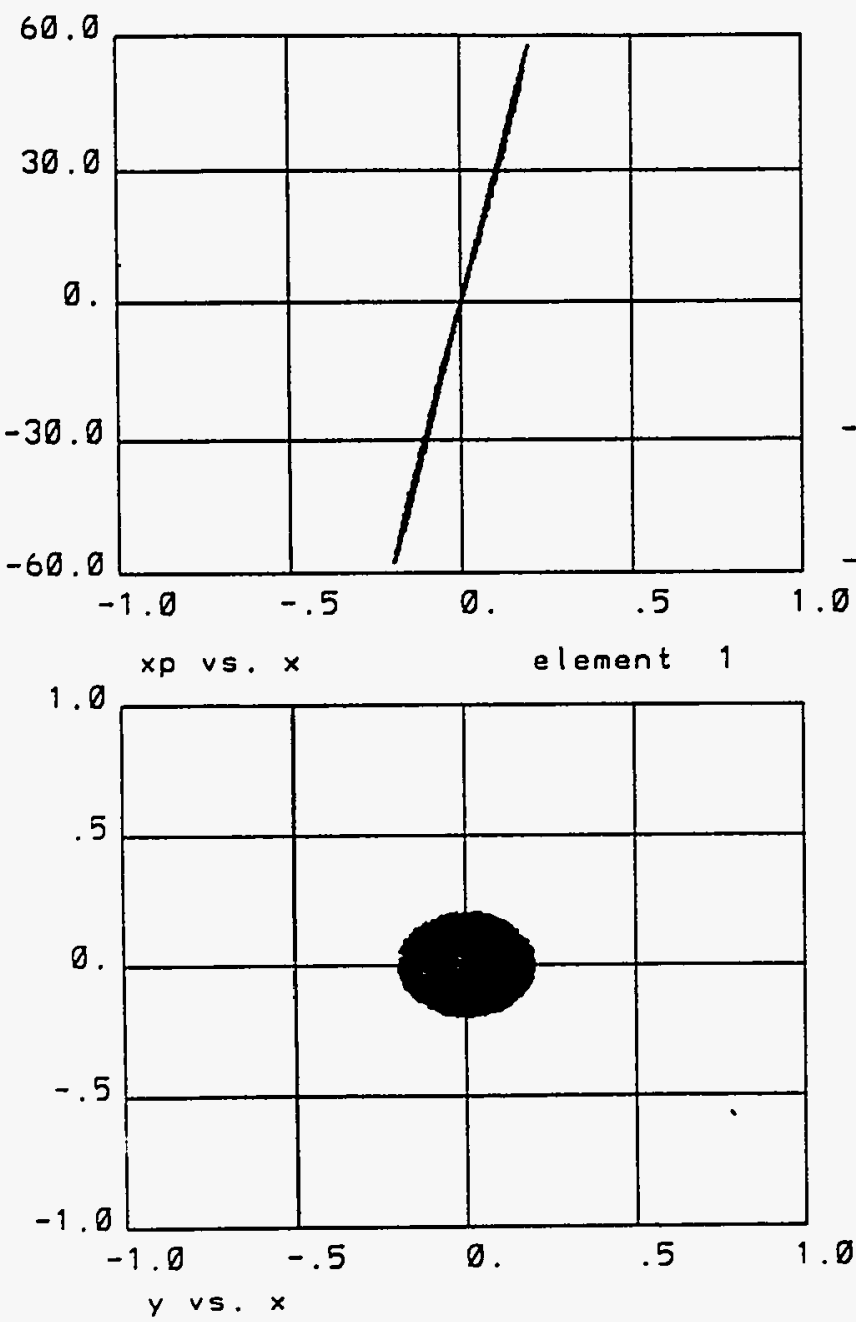

$10.9 \mathrm{~mm} !, 5.15 p s, 100 \mathrm{MV} / \mathrm{m}, \quad \mathrm{nC}, \mathrm{phi}=43.0)$
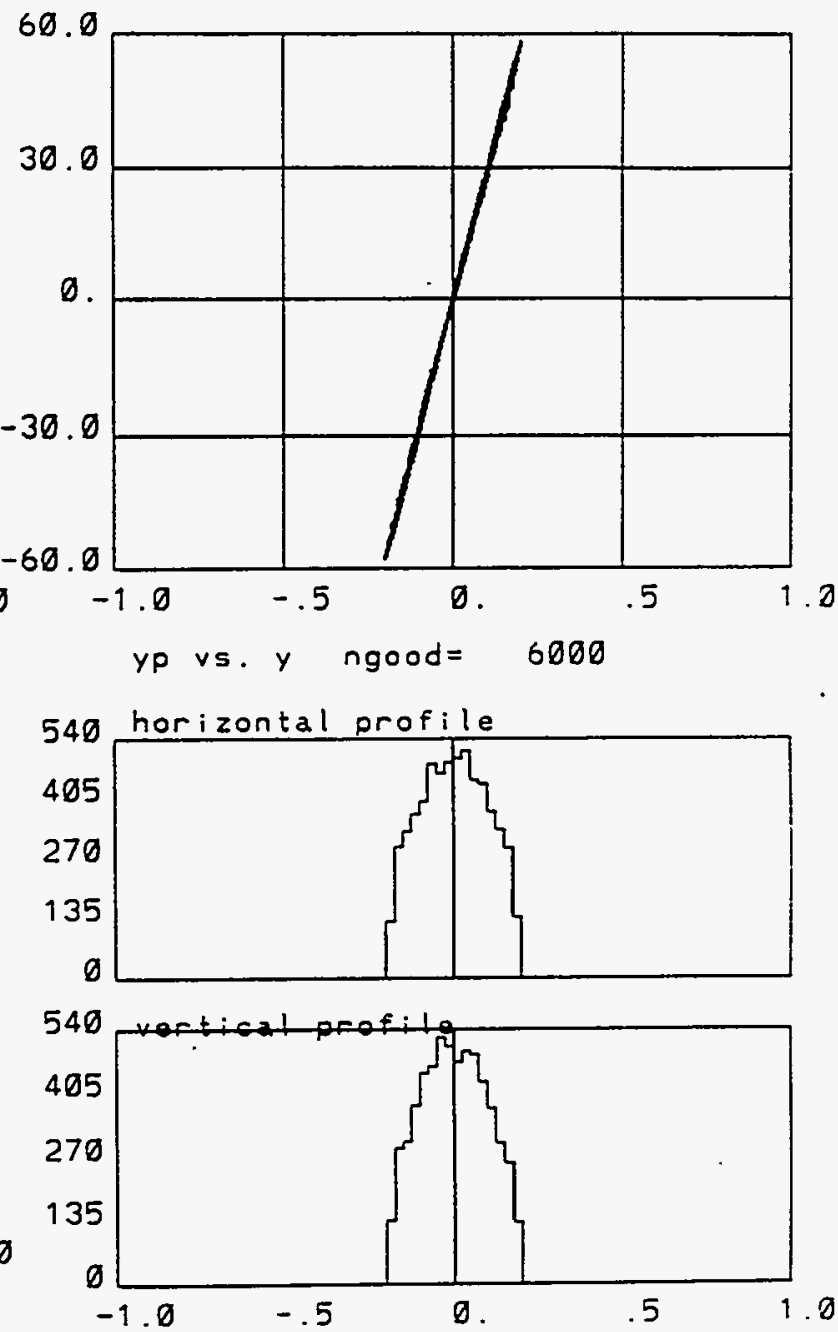

Figure $8-1$ shows the beam profile at position $(n e=1) z=0$, with the Inline-Injection system of Solenoid + Gun + Solenoid combination inline with the linac. With Solenoid current of $I=2500 \mathrm{amp}$. 
Figure $8-2$ shows the beam profile at position $(n e=2) z=2.6$, with the Inline-Injection system of Solenoid + Gun + Solenoid combination inline with the linac. With Solenoid current of $I=2500 \mathrm{amp}$.

- BNL RF GUN
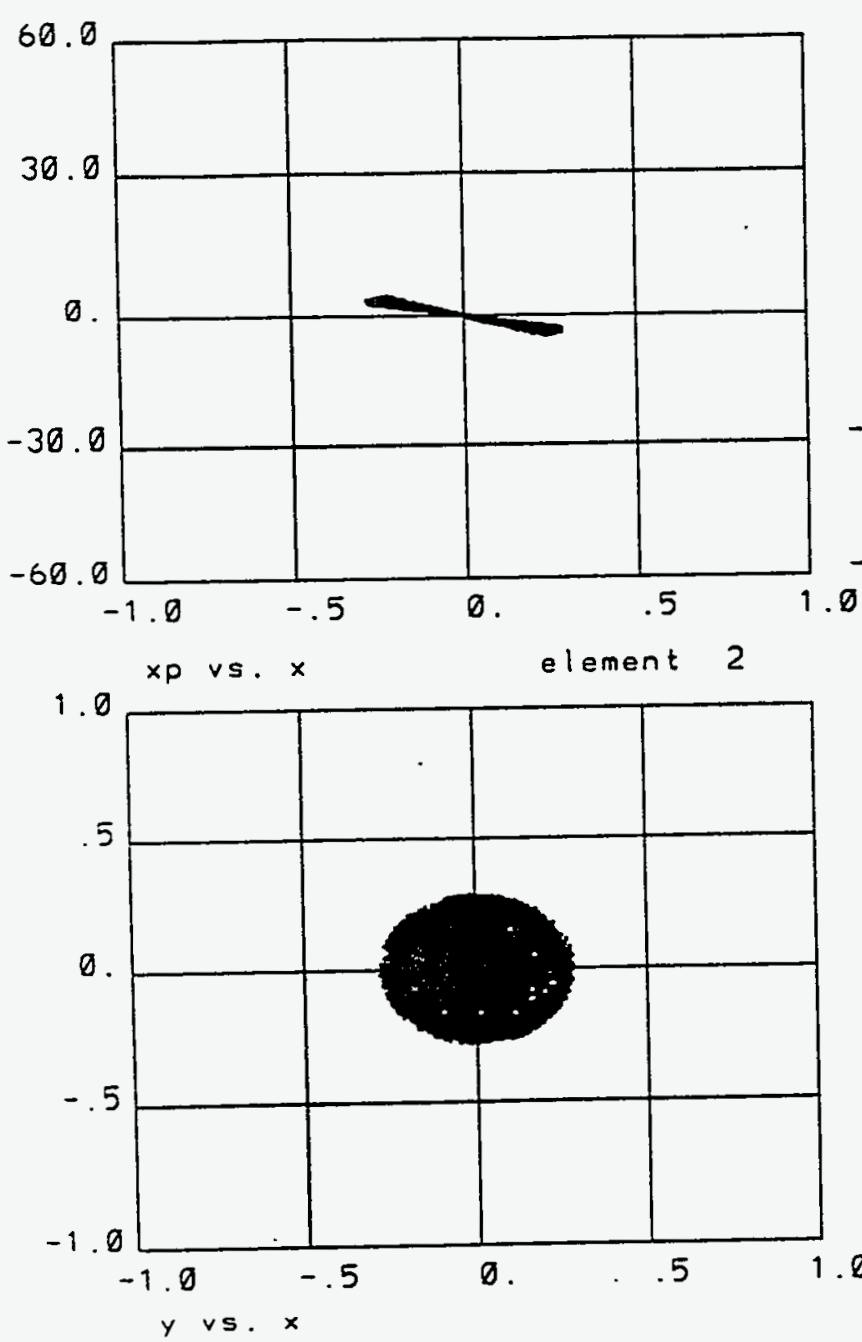

$(0.9 \mathrm{~mm}), 5.15 \mathrm{ps} .100 \mathrm{MV} / \mathrm{m}, 1 \mathrm{nC}, \mathrm{phi}=43.0)^{\circ}$
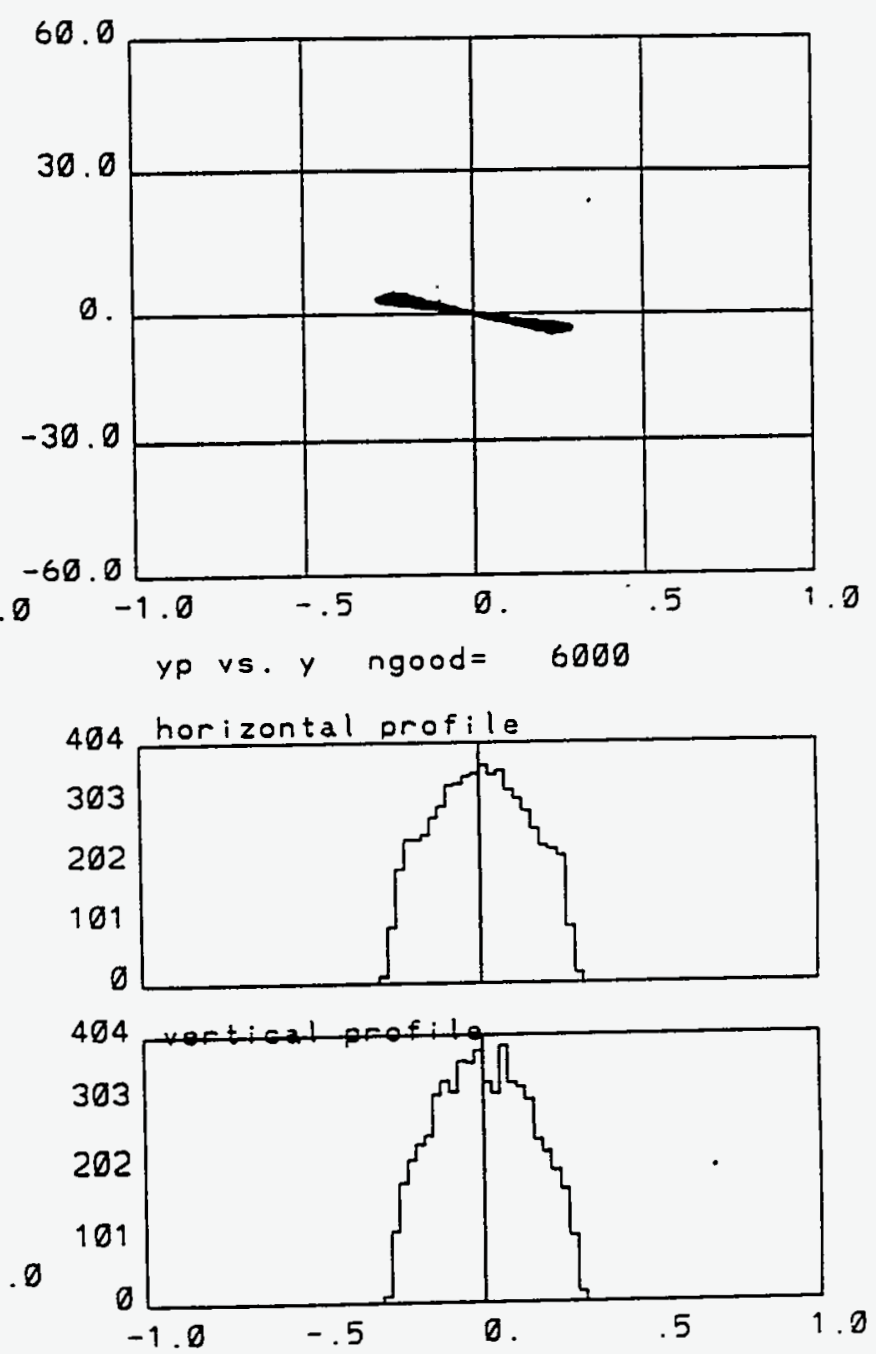
Figure $8-3$ shows the beam profile at position $(n e=3) z=5.2$, with the Inline-Injection system of Solenoid + Gun + Solenoid combination inline with the linac. With Solenoid current of $I=2500$ amp.

'BNL RF GUN

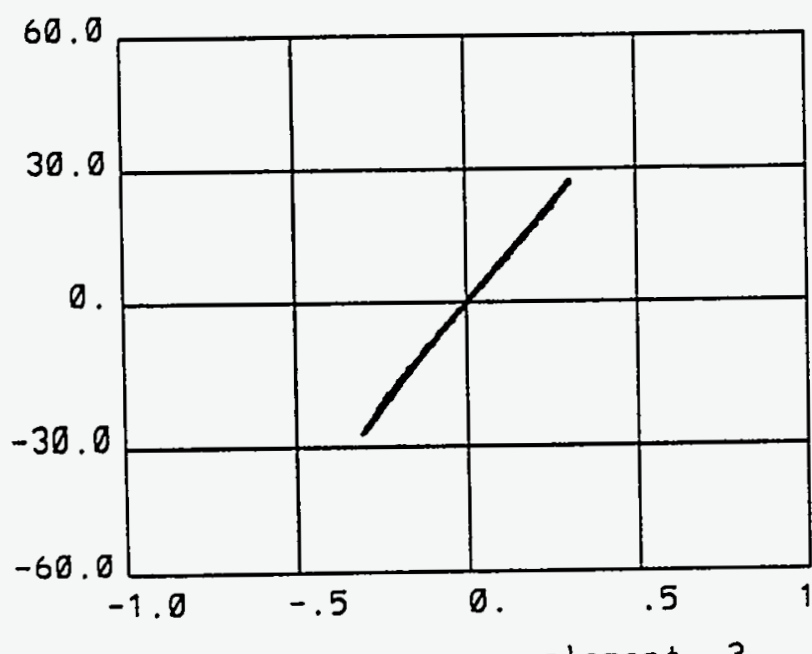

$x$ vs. $x$ element 3

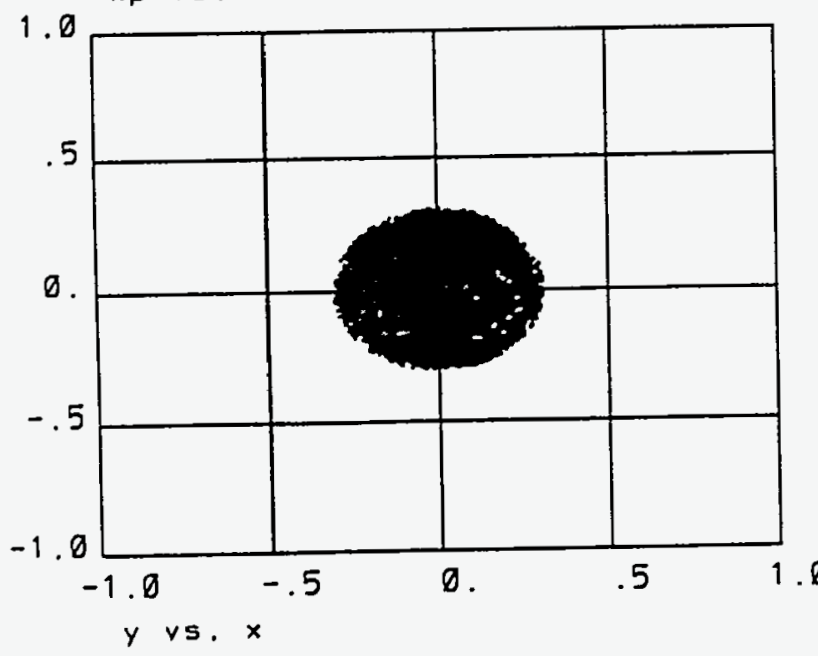

$(0.9 \mathrm{~mm}), 5.15 \mathrm{ps}, 100 \mathrm{MV} / \mathrm{m}, \quad \mathrm{nC}, \mathrm{phi}=43.0)$
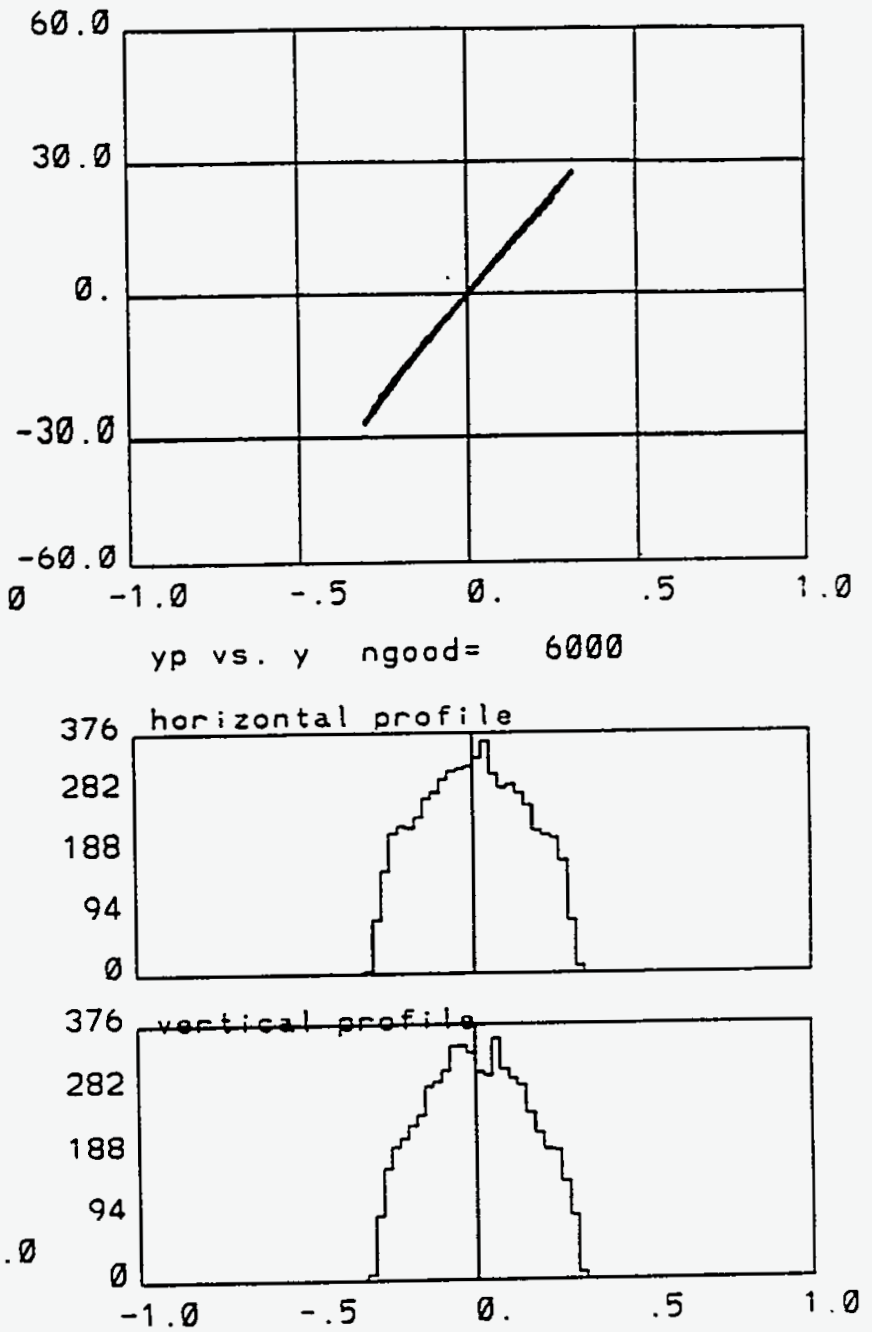
Figure 8-4 shows the beam profile at position $(n e=4) z=7.9$, with the Inline-Injection system of Solenoid + Gun + Solenoid combination inline with the linac. With Solenoid current of $I=2500$ amp.

- BNL RF GUN
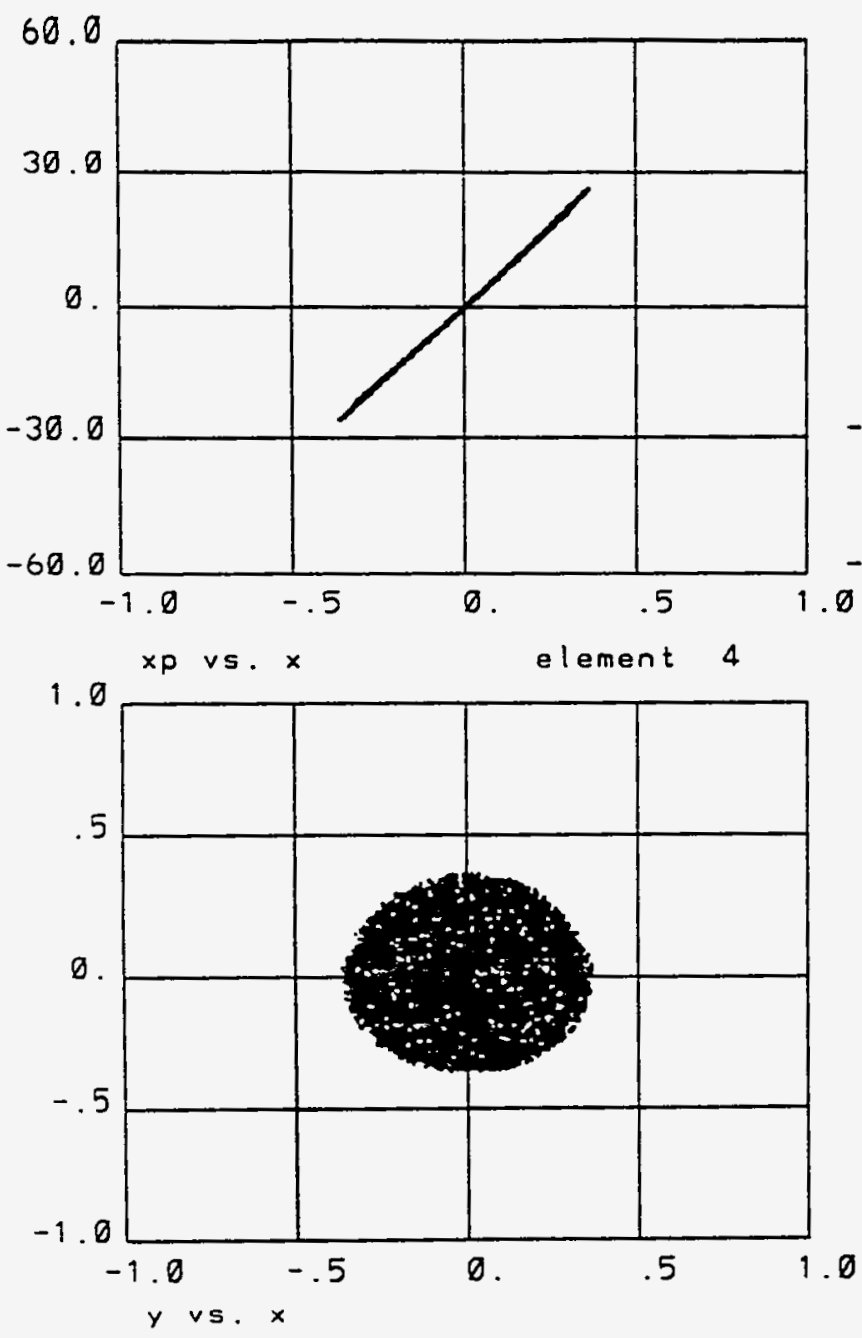

$15.15 p s .100 \mathrm{MV} / \mathrm{m}, 1 \mathrm{nC}, \mathrm{phi}=43.01^{\circ}$

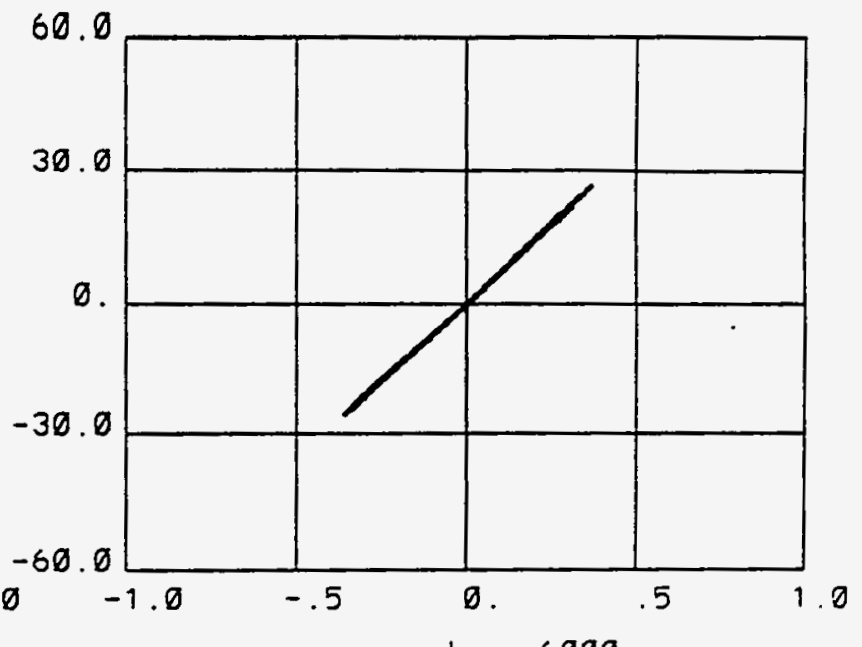

yp vs. y ngood $=6000$
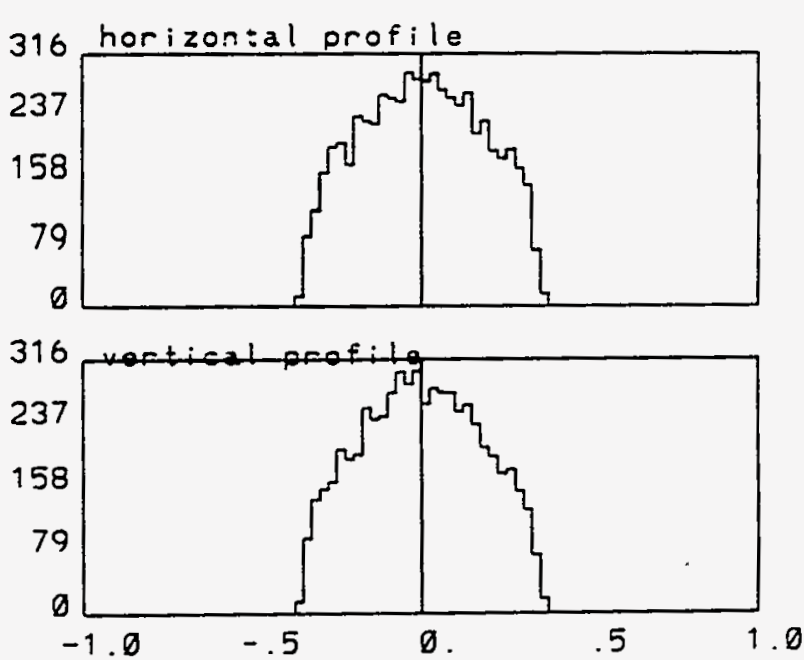
Figure $8-5$ shows the beam profile at position $(n e=5) z=10$, with the Inline-Injection system of Solenoid + Gun + Solenoid combination inline with the linac. With Solenoid current of $I=2500 \mathrm{amp}$.

BNL RF GUN $\quad 1,5.150 \mathrm{~s}, 100 \mathrm{MV} / \mathrm{m}, 1 \mathrm{nC}, \mathrm{phi}=43.01$.
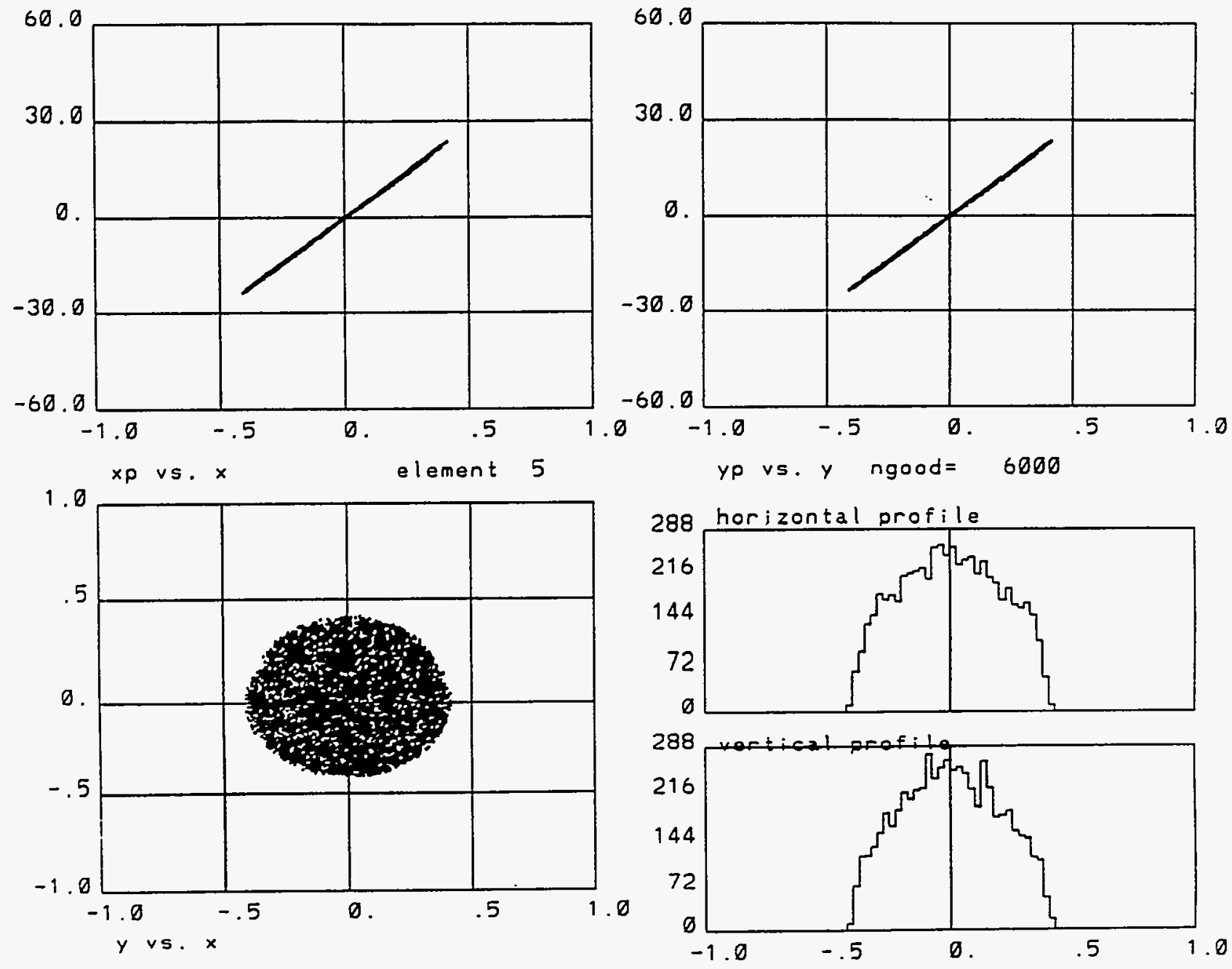
Figure $8-6$ shows the beam profile at position $(n e=10) \quad z=20$, with the Inline-Injection system of Solenoid + Gun + Solenoid combination inline with the linac. With Solenoid current of $I=2500$ amp.

- BNL RF GUN
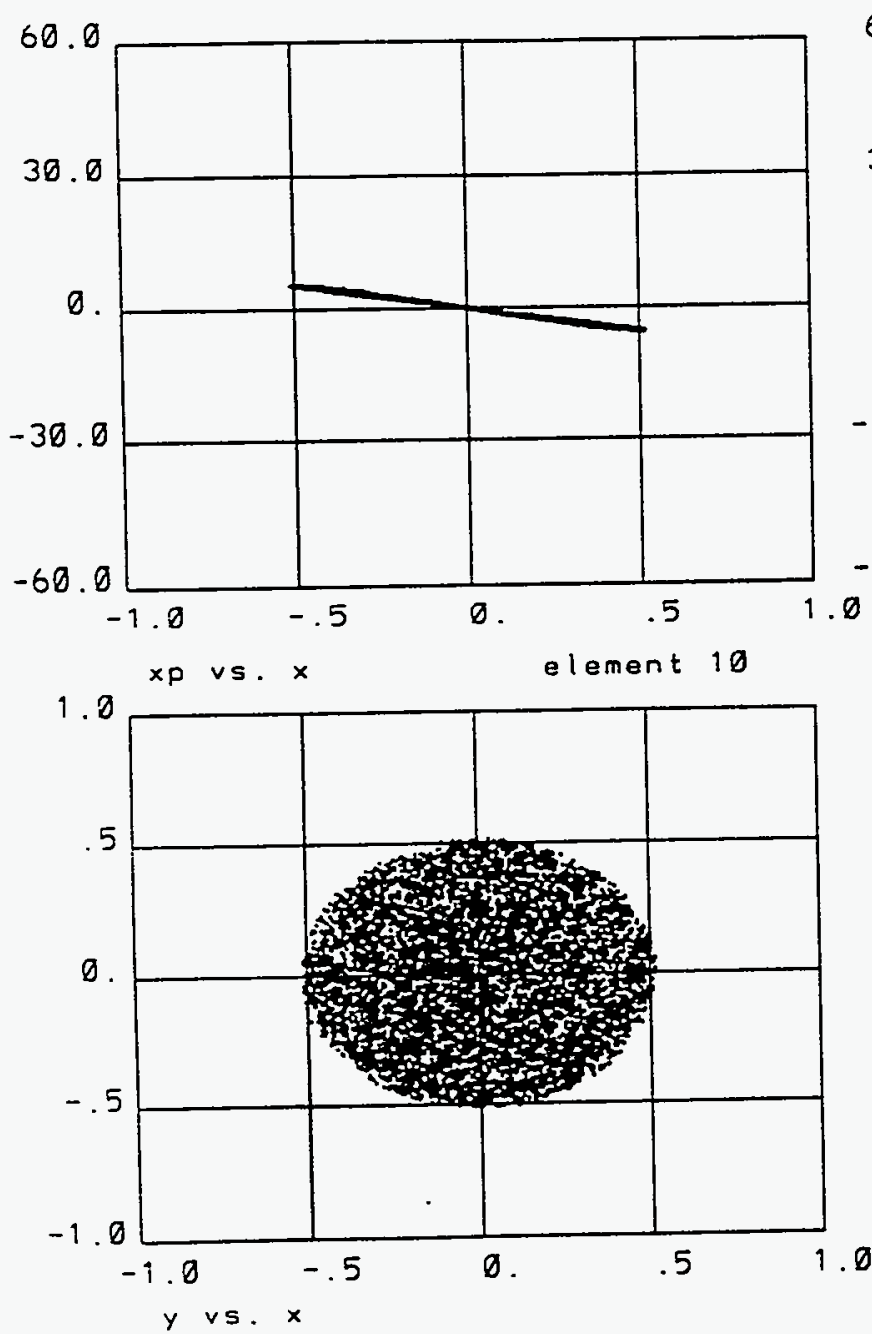

$1,5.15 p s, 100 \mathrm{MV} / \mathrm{m} .1 \mathrm{nC}, \quad$ ph $i=43.01^{\circ}$

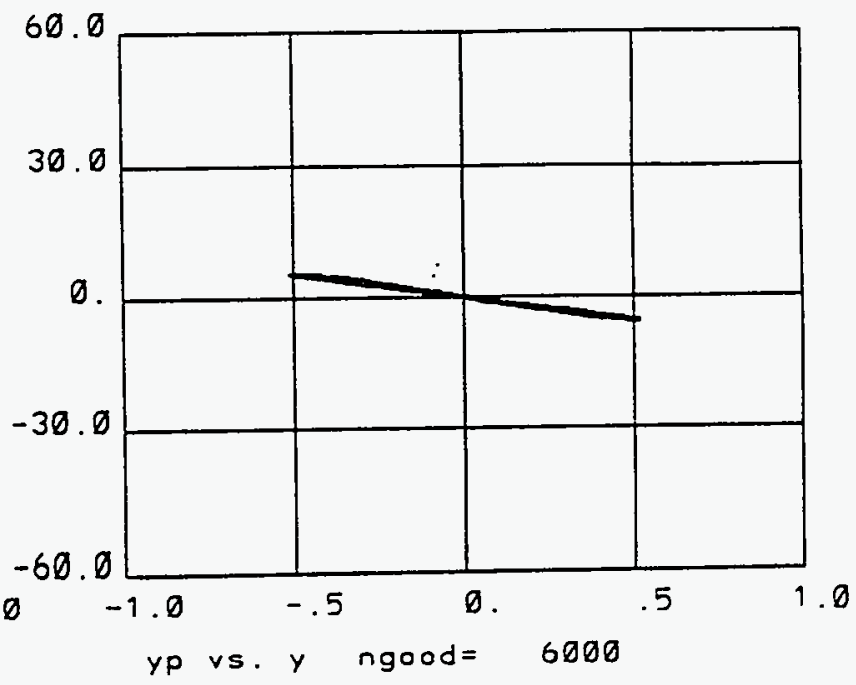

228 horizontal profile
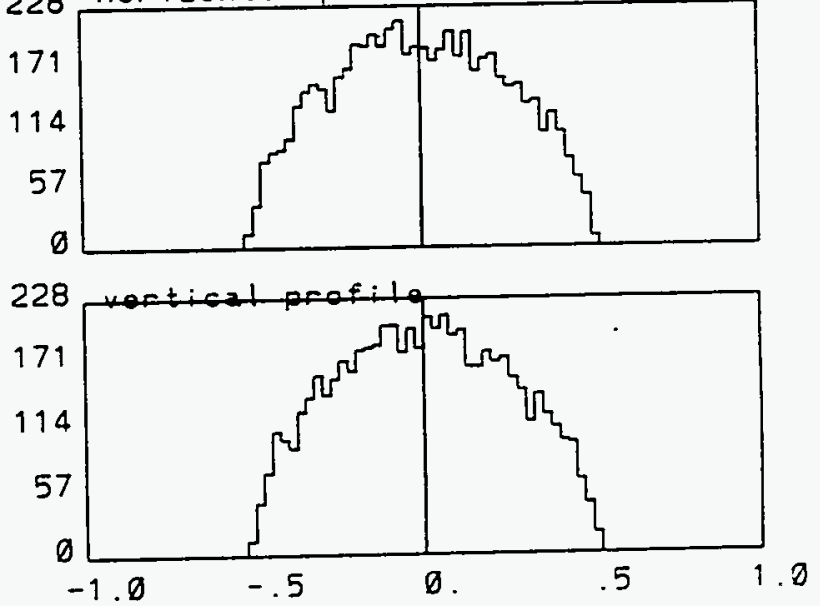
Figure $8-7$ shows the beam profile at position $(n e=20) \quad z=40$, with the Inline-Injection system of Solenoid + Gun + Solenoid combination inline with the linac. With Solenoid current of $I=2180 \mathrm{amp}$.

'BNL RF GUN
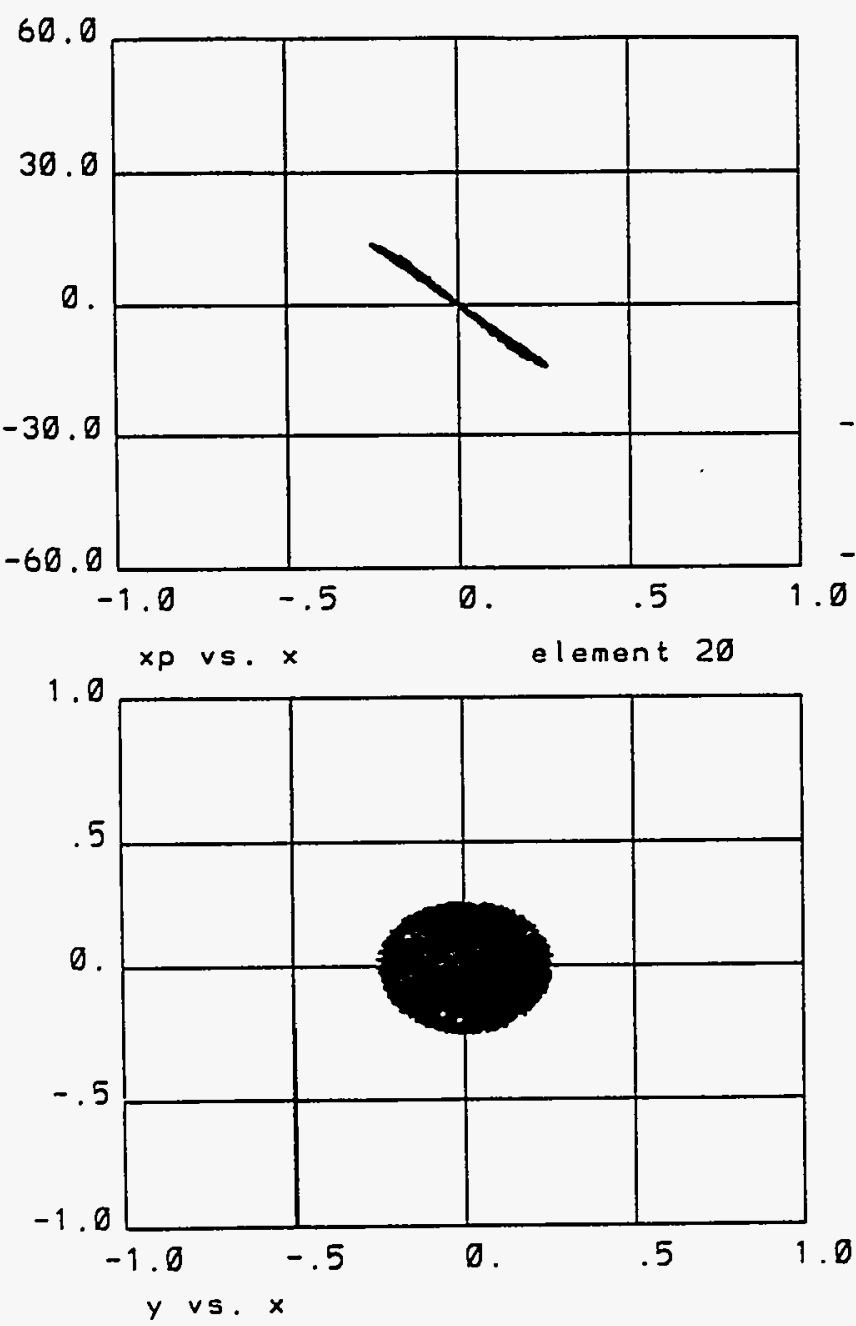

$(0.9 \mathrm{~mm}), 5.15 \mathrm{ps} .100 \mathrm{MV} / \mathrm{m}, 1 \mathrm{nC}, \mathrm{phi}=43.01$.

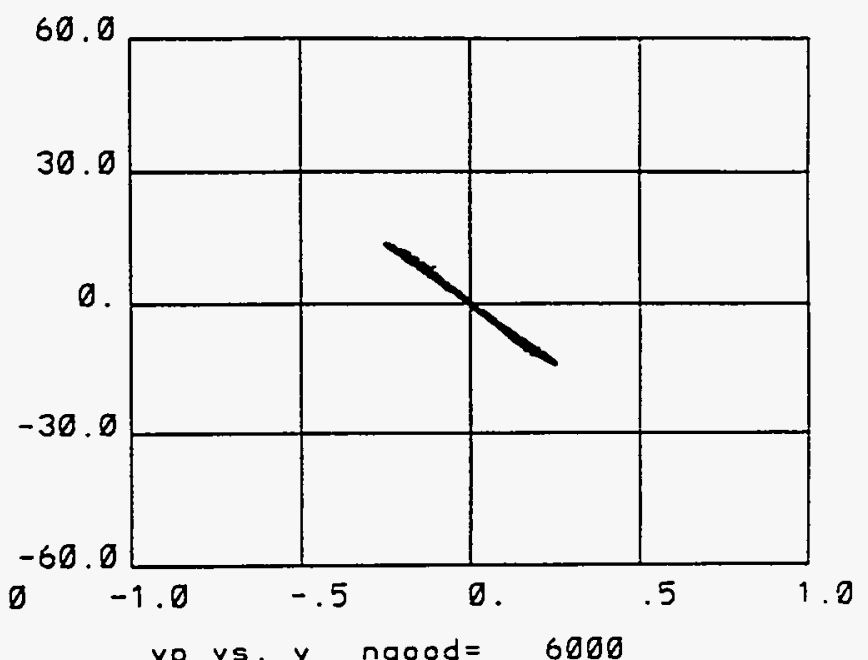

yp vs. y ngood $=6000$
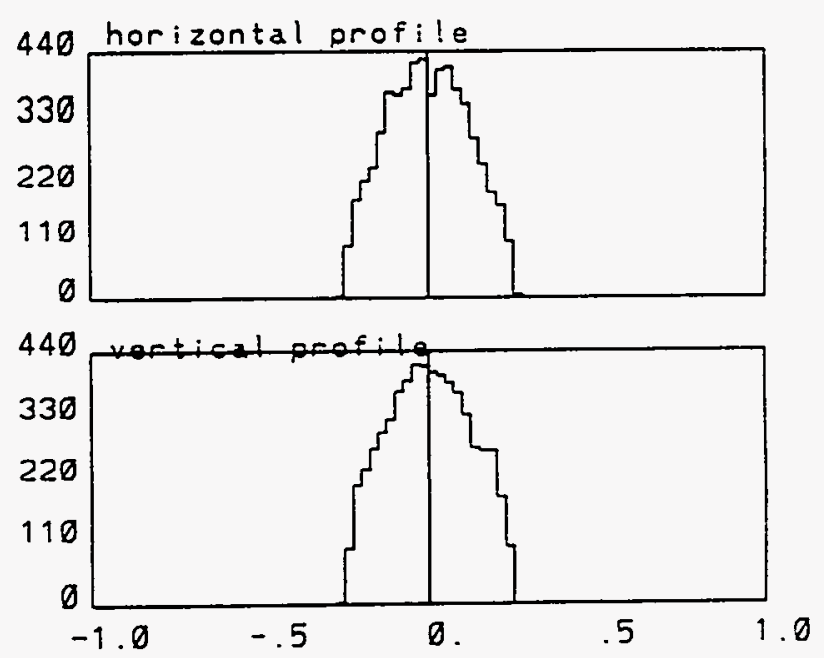
Figure $8-8$ shows the beam profile at position $(n e=27) \quad z=58$, with the Inline-Injection system of Solenoid + Gun + Solenoid combination inline with the linac. With Solenoid current of $I=2500 \mathrm{amp}$.

- BNL RF GUN
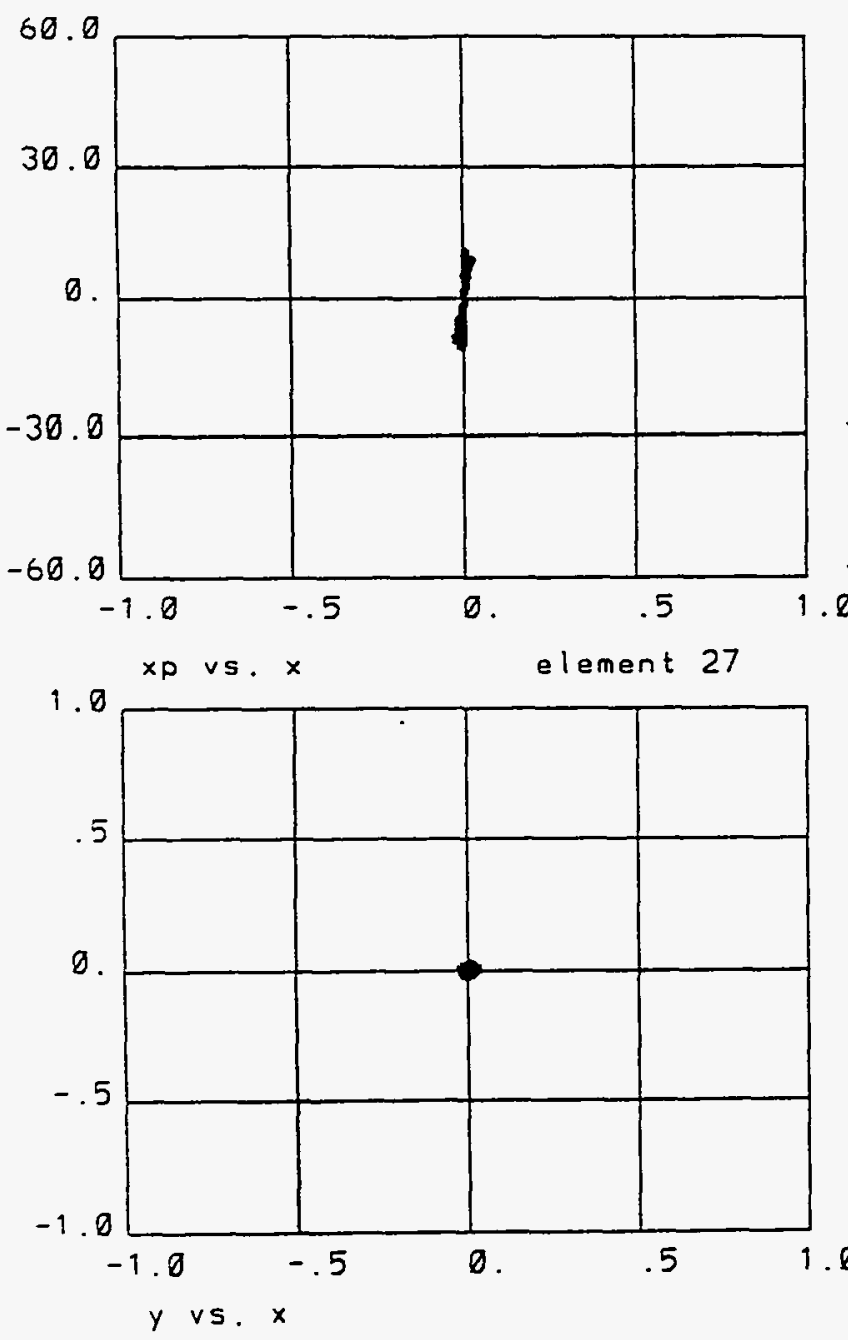

$(0.9 \mathrm{~mm}), 5.15 \mathrm{ps} .100 \mathrm{MV} / \mathrm{m}, 1 \mathrm{nC}$, phi $=43.0)^{\circ}$

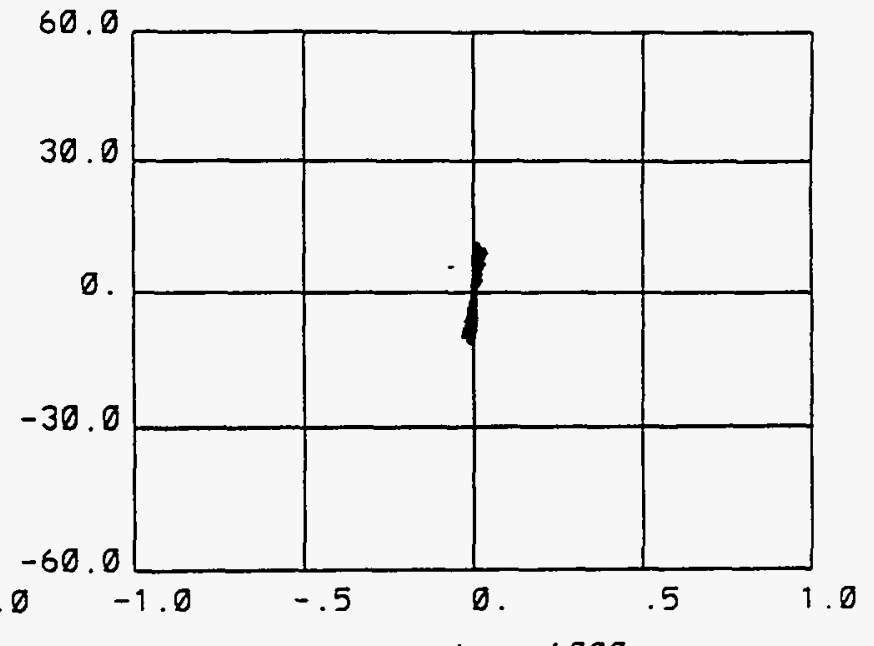

yo vs. y ngood $=6000$

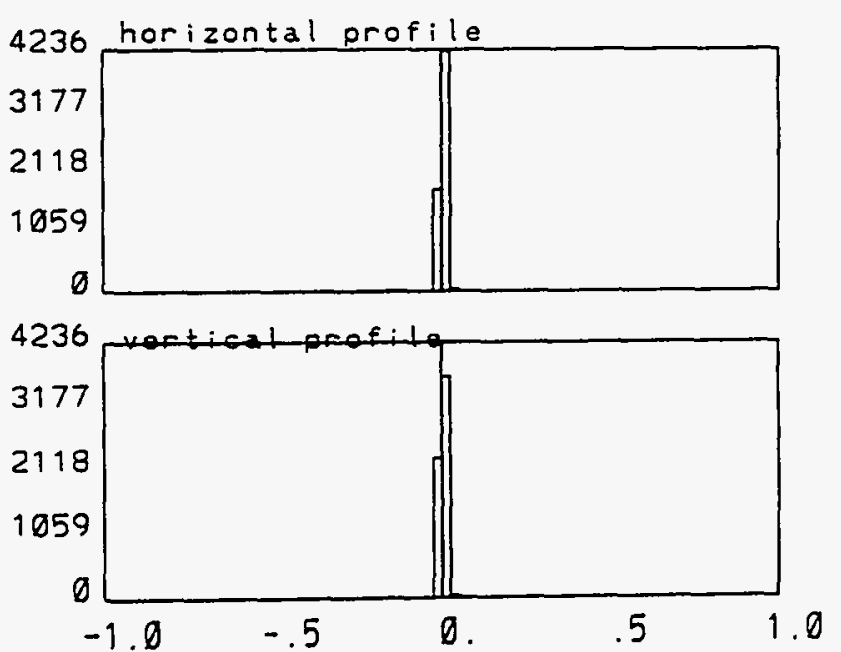


Figure $8-9$ shows the beam profile at position $(n e=30) z=70$, with the Inline-Injection system of Solenoid + Gun + Solenoid combination inline with the linac. With Solenoid current of $\mathrm{I}=2500 \mathrm{amp}$.

- BNL RF GUN
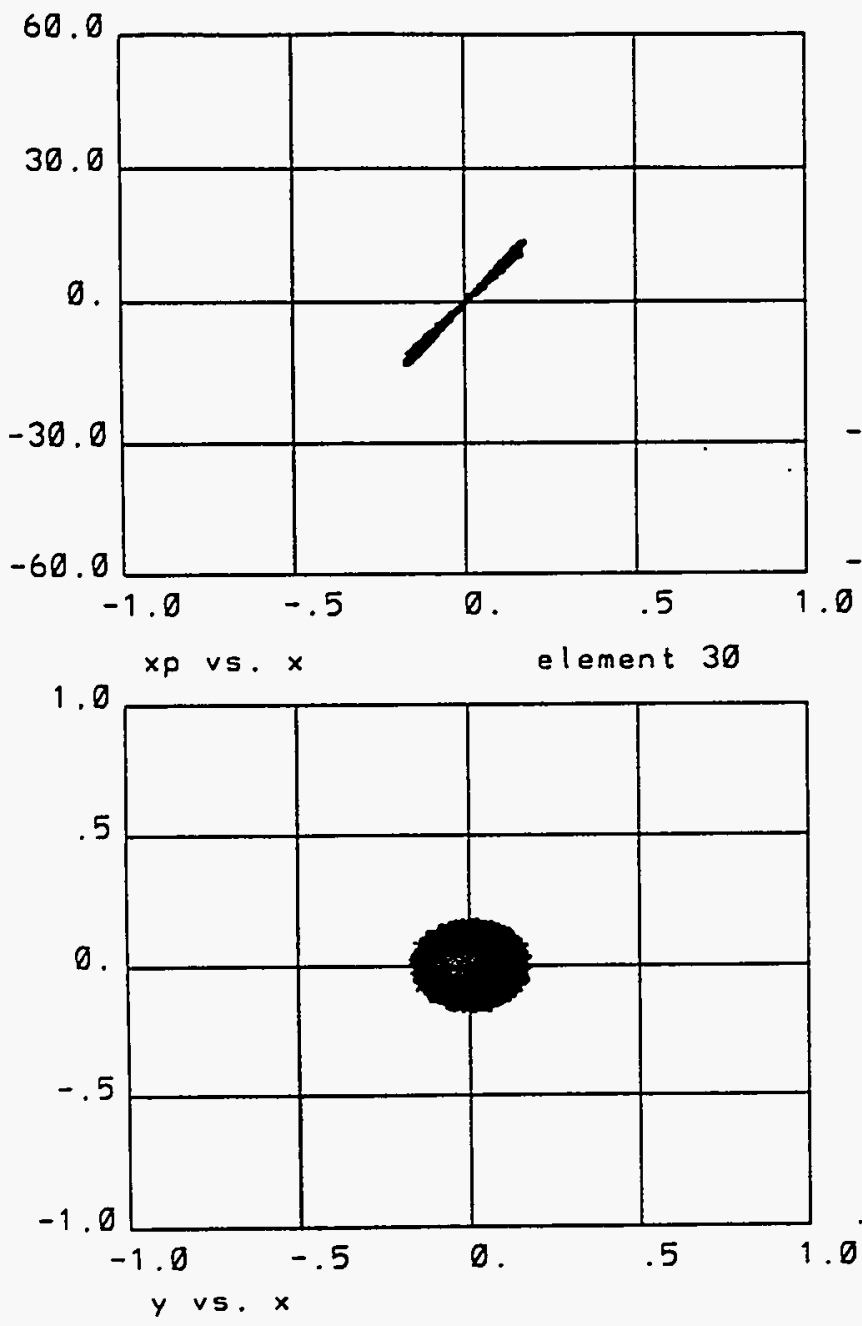

$(0.9 \mathrm{~mm}), 5.15 \mathrm{ps}, 100 \mathrm{MV} / \mathrm{m}, 1 \mathrm{nC}, \mathrm{phi}=43.0)$
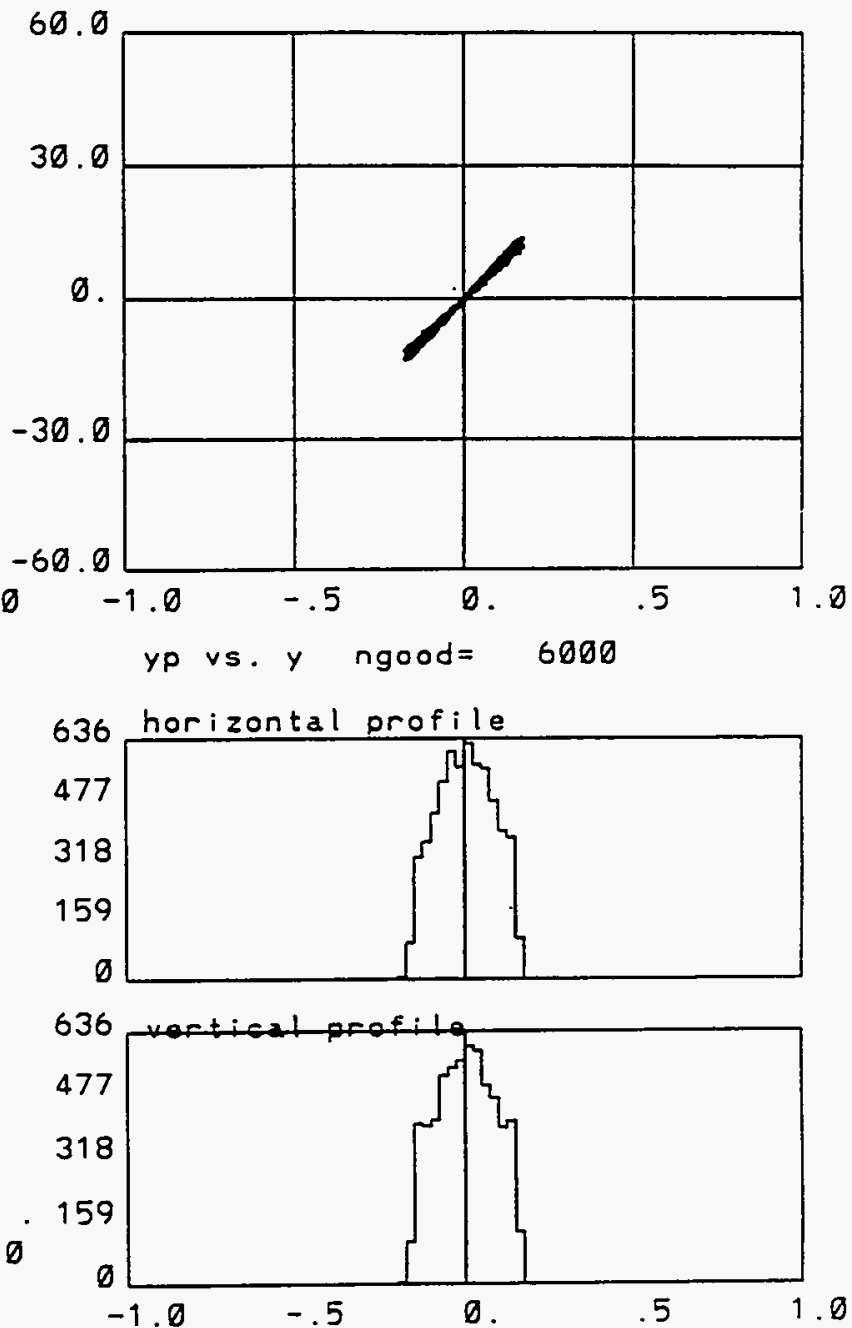
Figure $8-10$ shows the beam profile at position $(n e=35) z=95$, with the Inline-Injection system of Solenoid + Gun + Solenoid combination inline with the linac. With Solenoid current of $I=2500 \mathrm{amp}$.

- BNL RF GUN

60.9

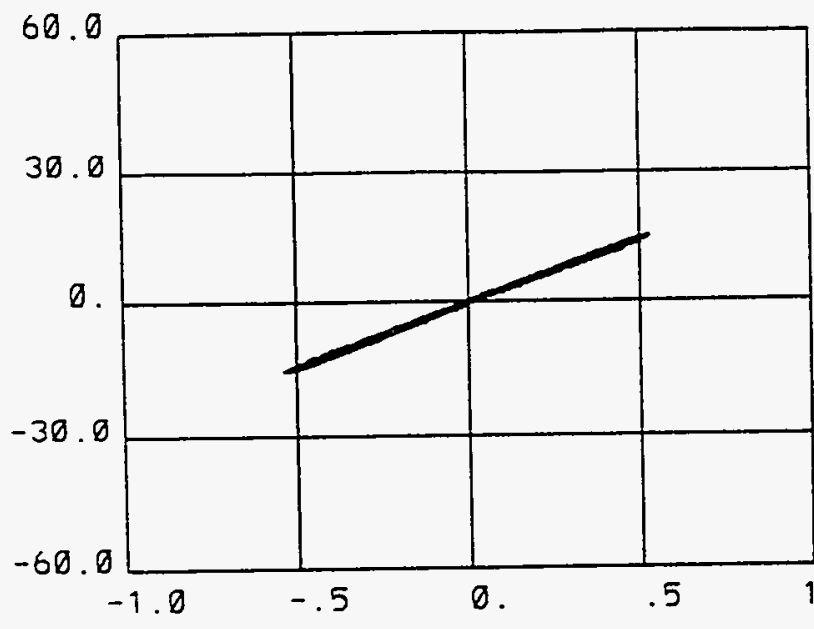

xp vs. $x$ element 35

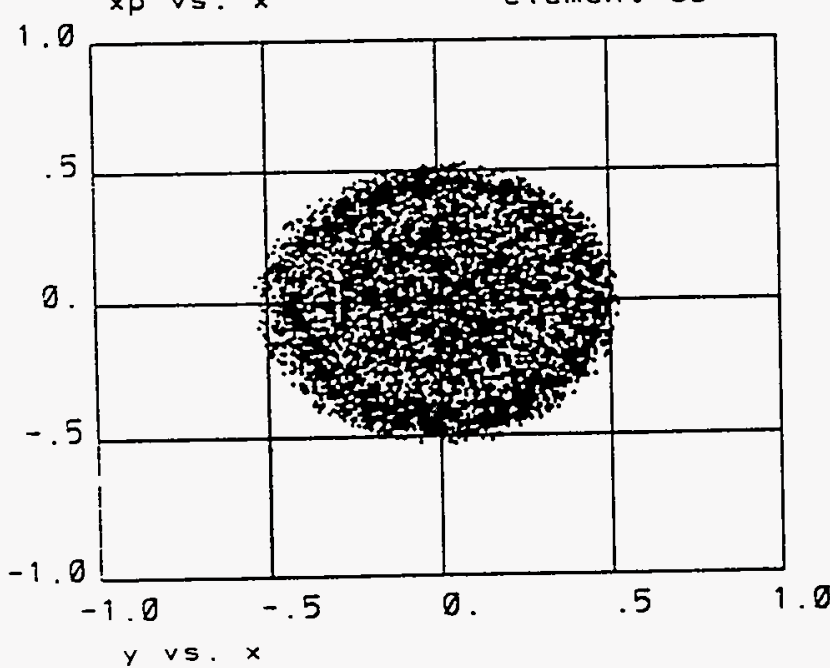

$(0.9 \mathrm{~mm}), 5.15 p s, 100 \mathrm{MV} / \mathrm{m} .1 \mathrm{nC}, \mathrm{phi}=43.0)^{\prime}$
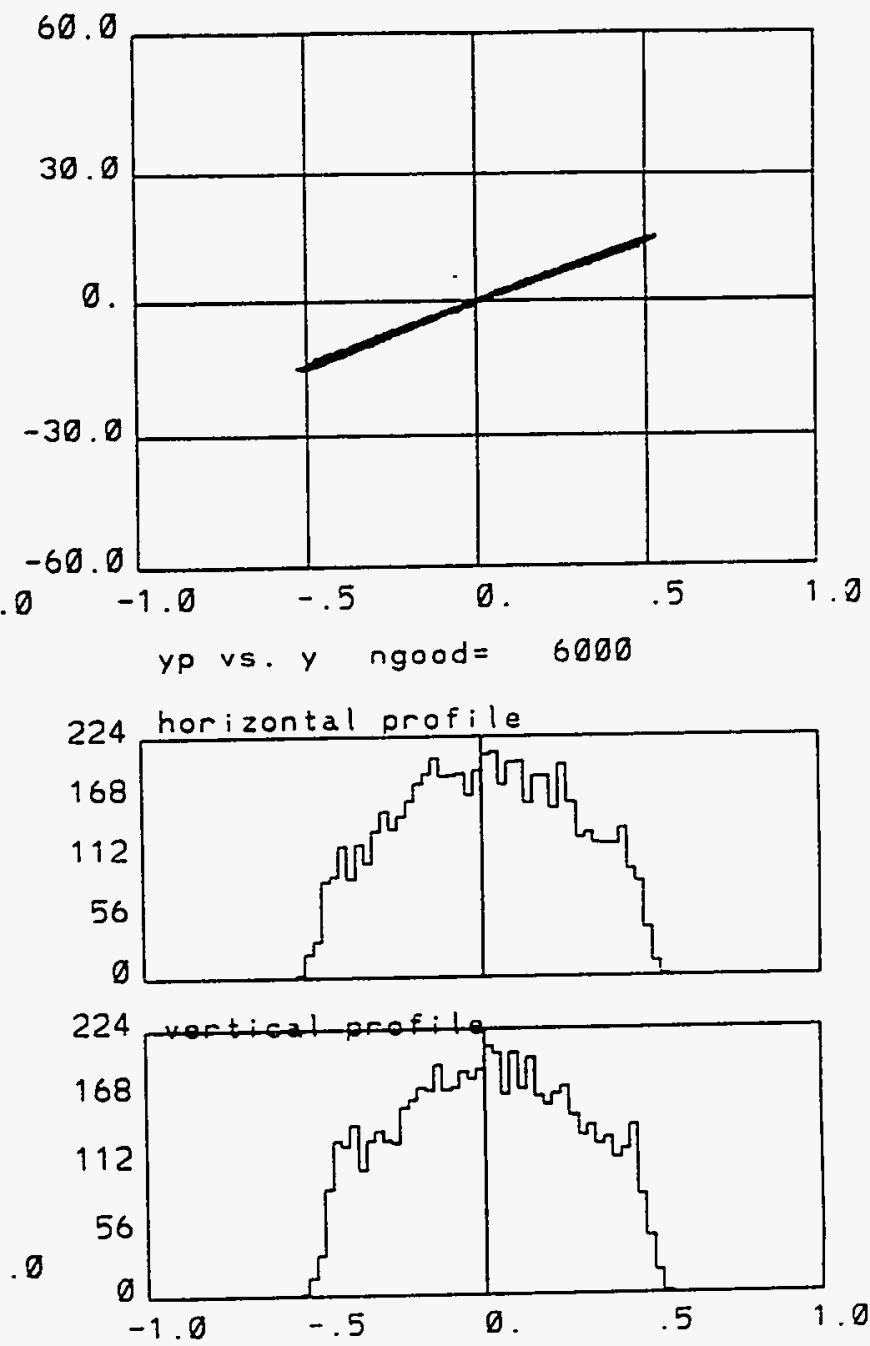
Figures 9-1 to 9-9 shows the beam profile at various positions (distance $z$ from the cathode), with the Inline-Injection system of Solenoid + Gun + Solenoid combination inline with the linac. With Solenoid current of I=2600 amp.

- BNL RF GUN
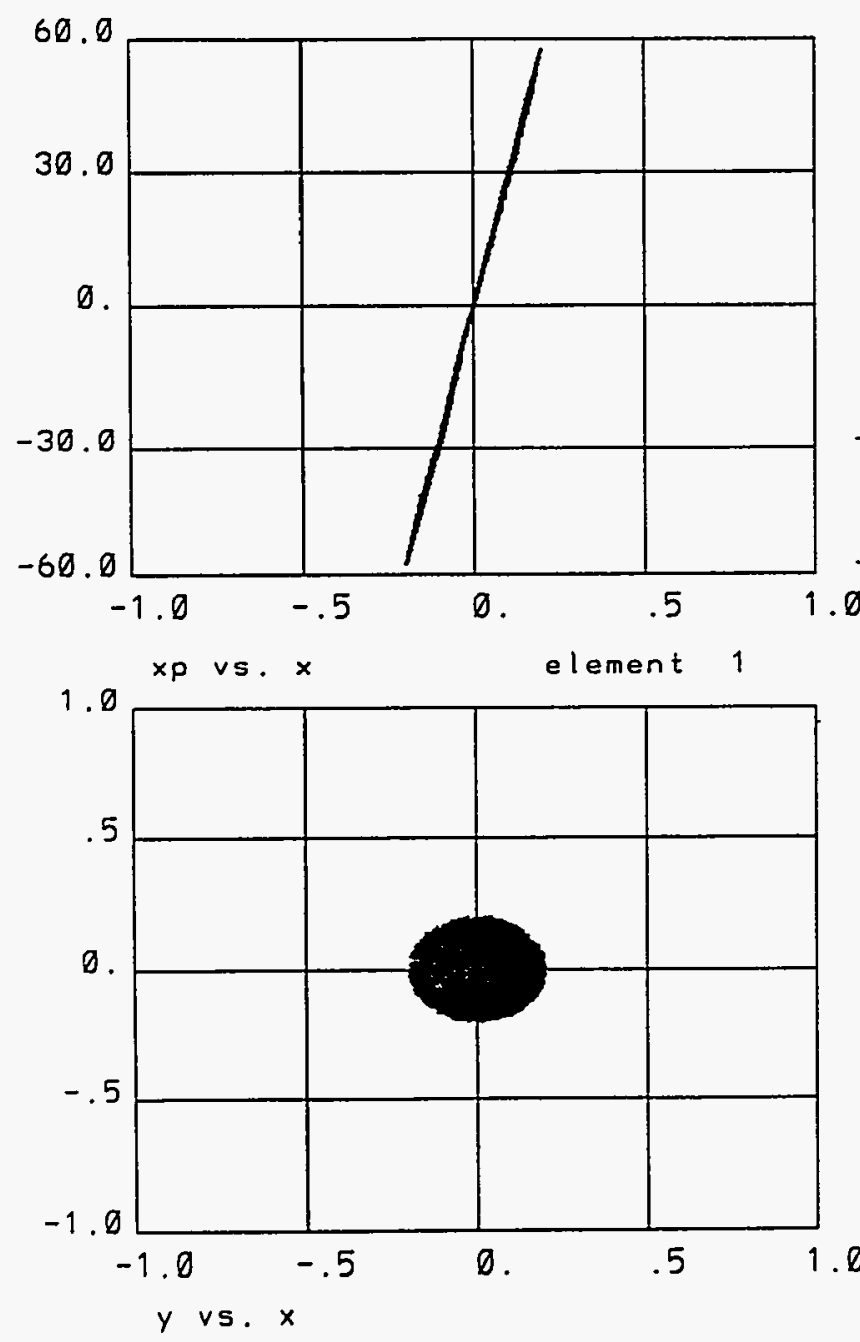

$15.15 p s G(10), 1 \mathrm{nC}$, phi $=43.)^{\circ}$

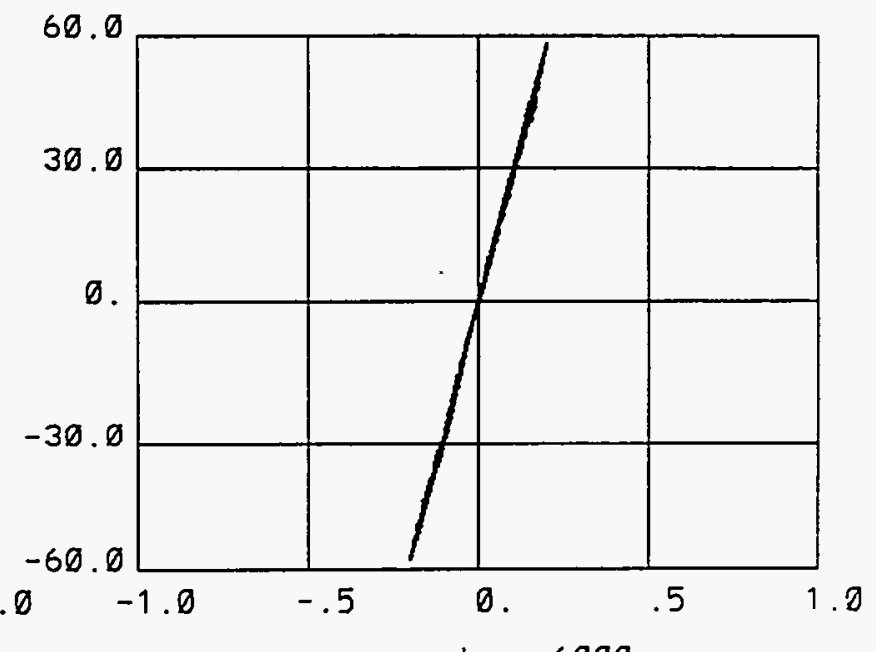

yp vs. y ngood $=6000$
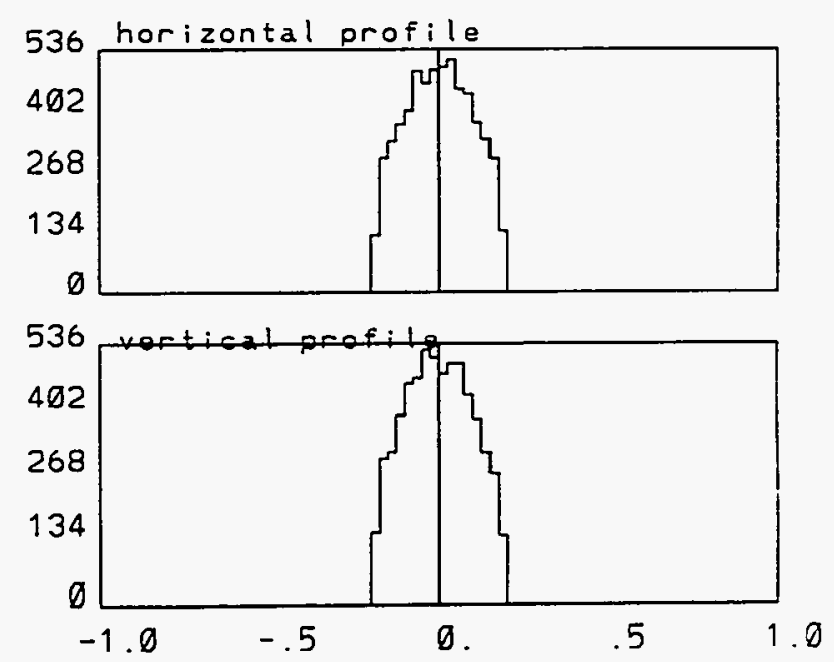

Figure $9-1$ shows the beam profile at position $(n e=1) z=0$, with the Inline-Injection system of Solenoid + Gun + Solenoid combination inline with the linac. With Solenoid current of $I=2600 \mathrm{amp}$. 
Figure $9-2$ shows the beam profile at position $(n e=2) z=2.6$, with the Inline-Injection system of Solenoid + Gun + Solenoid combination inline with the linac. With Solenoid current of $I=2600 \mathrm{amp}$.

- BNL RF GUN

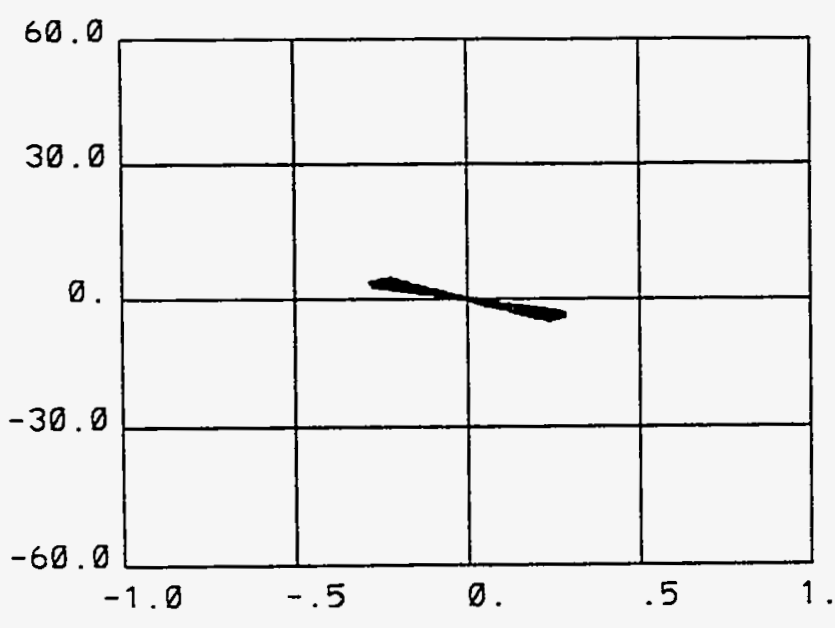

$x$ vs. $x$ element 2

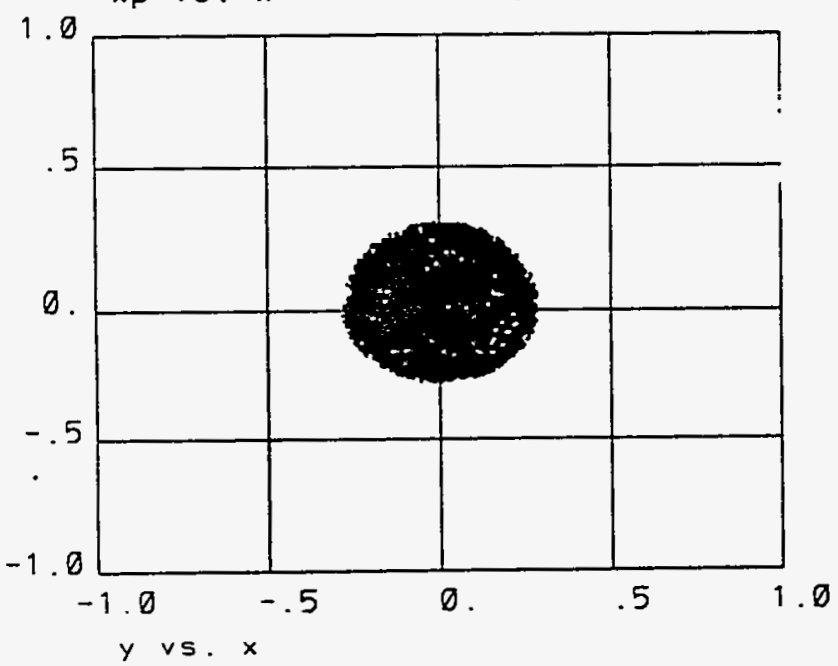

,5.15ps G(10),1 nC, phi $=43.1^{\circ}$
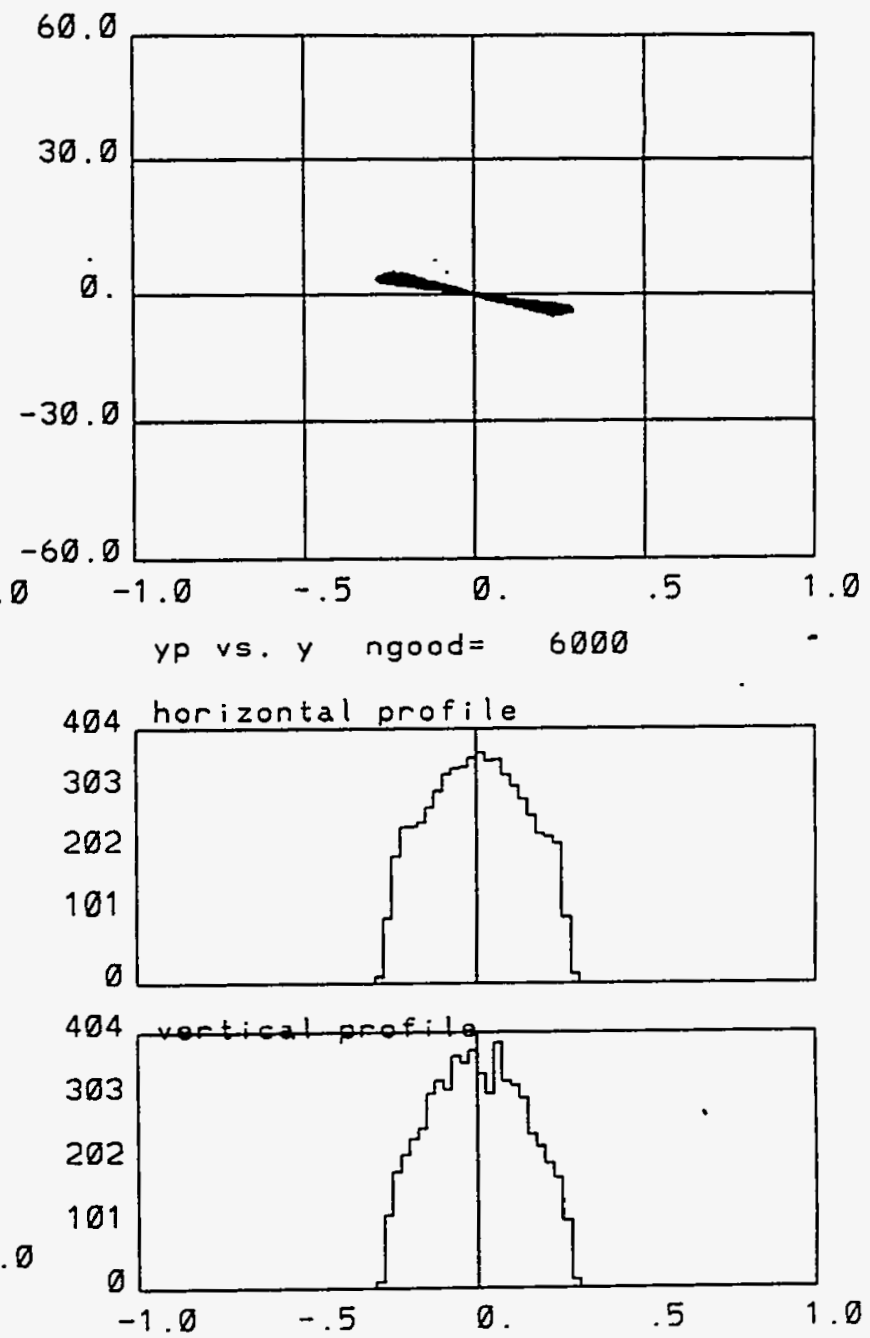
Figure $9-3$ shows the beam profile at position $(n e=3) z=5.2$, with the Inline-Injection system of Solenoid + Gun + Solenoid combination inline with the linac. With Solenoid current of $I=2600 \mathrm{amp}$.

'BNL RF GUN

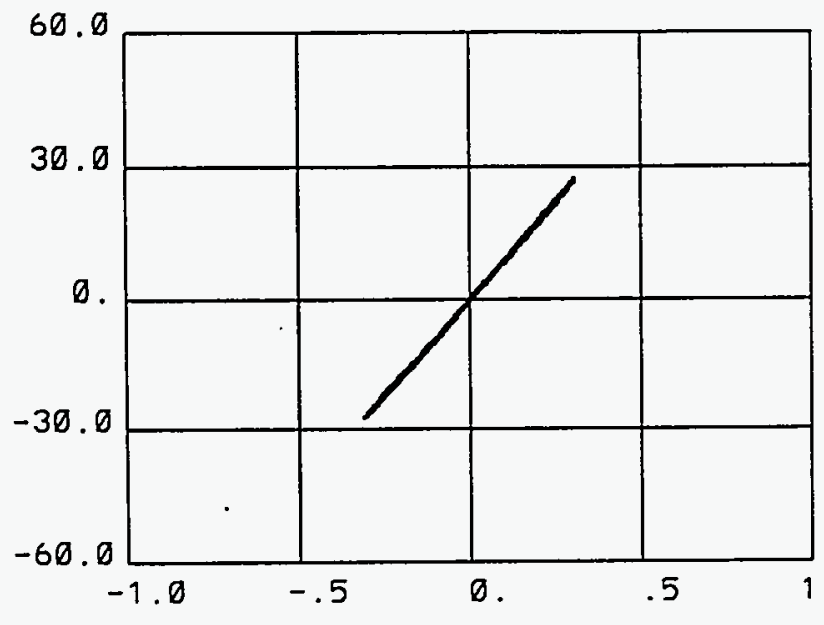

xp vs. $x \quad$ element 3

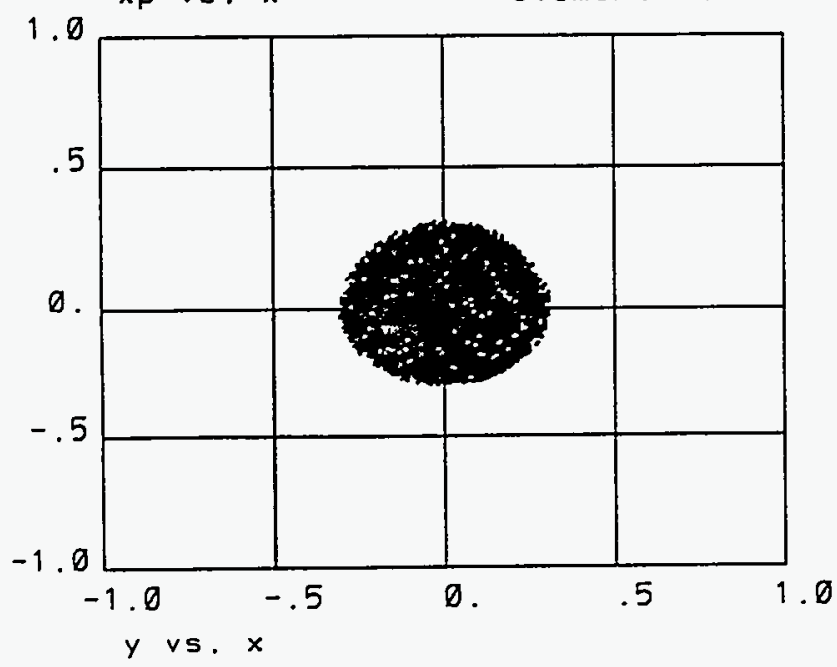

15.15ps $G(10), 1 \mathrm{nC}$, phi=43.1.
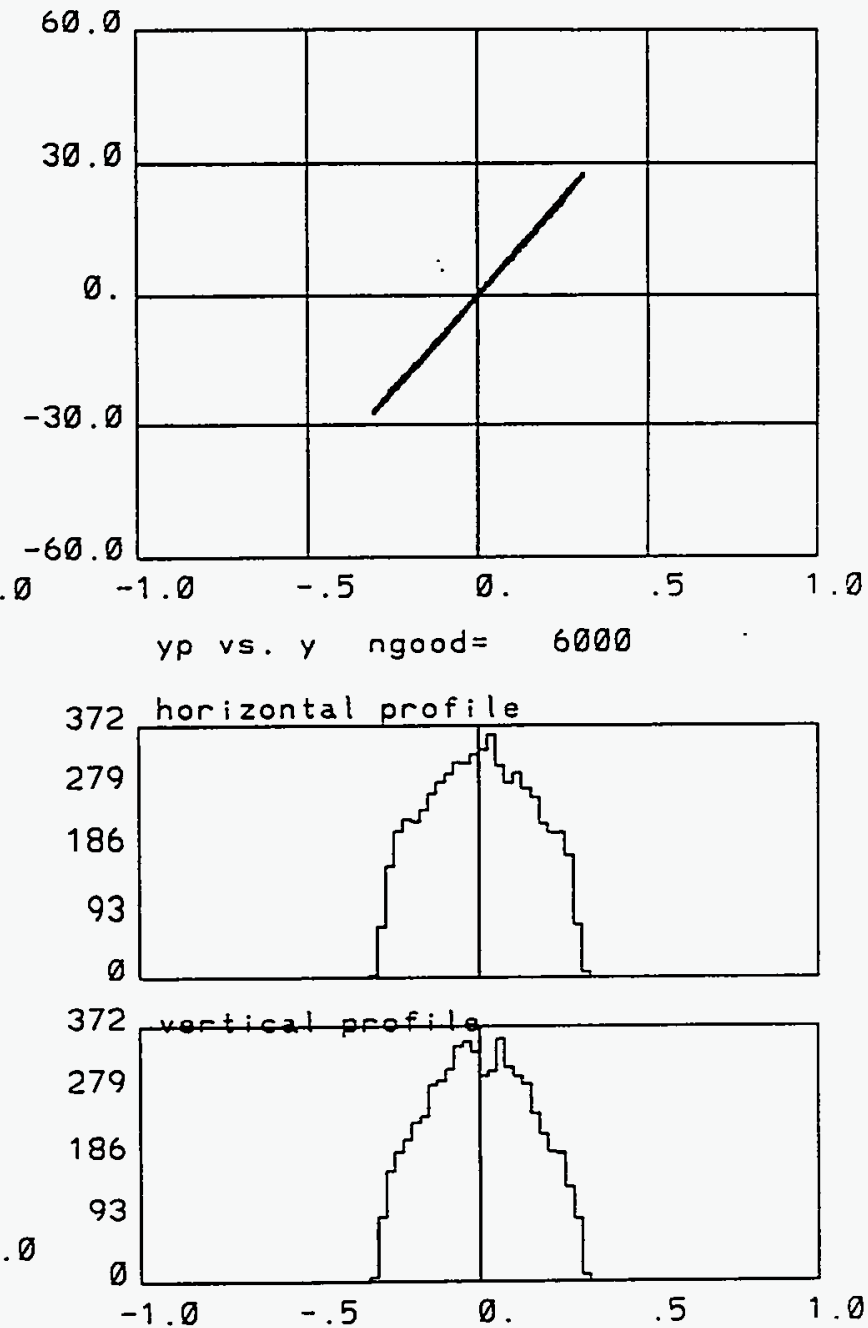
Figure 9-4 shows the beam profile at position $(n e=4) \quad z=7.9$, with the Inline-Injection system of Solenoid + Gun + Solenoid combination inline with the linac. With Solenoid current of $I=2600 \mathrm{amp}$.

- BNL RF GUN
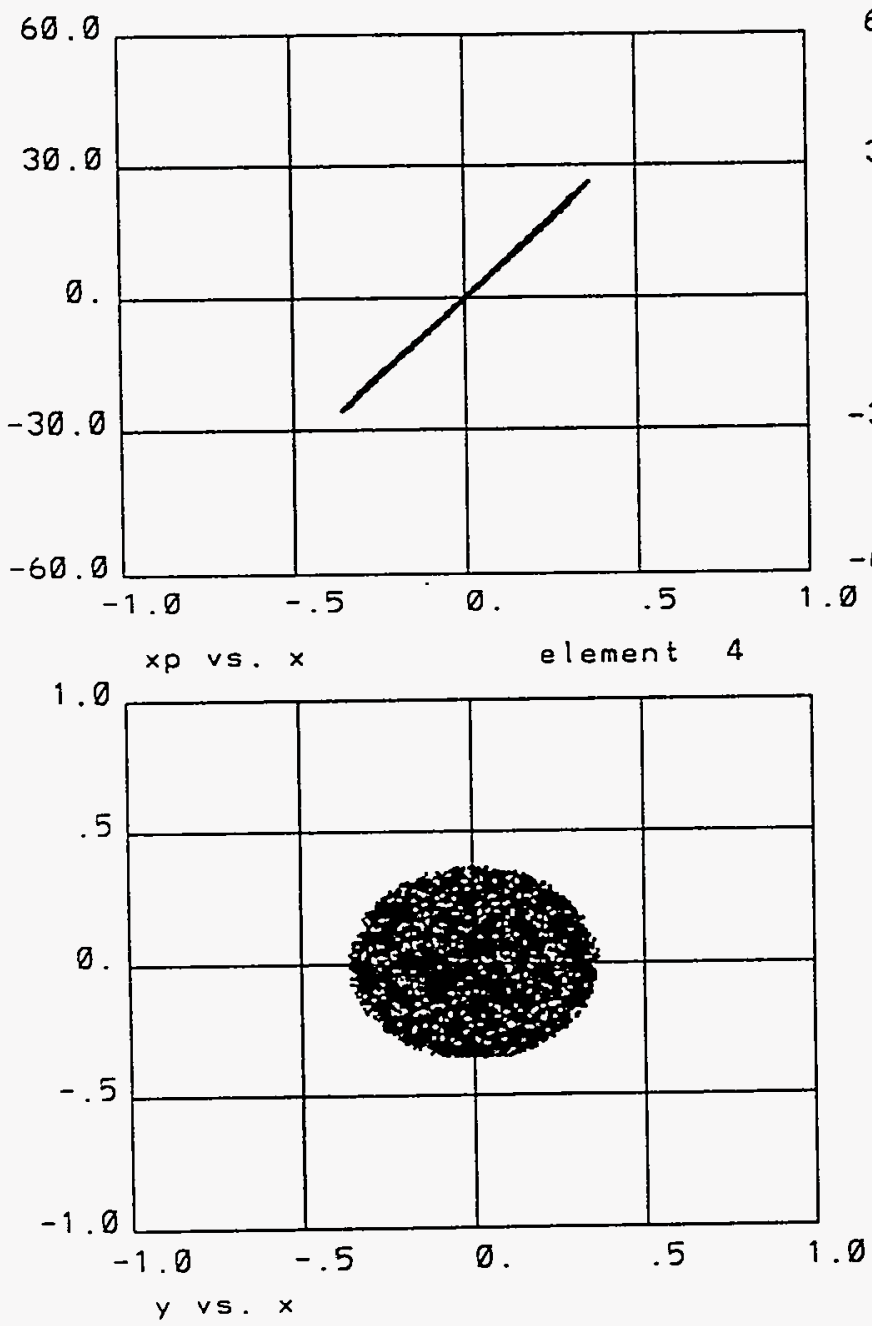

$.5 .15 p s G(10), 1 \mathrm{nC}$, phi=43.).
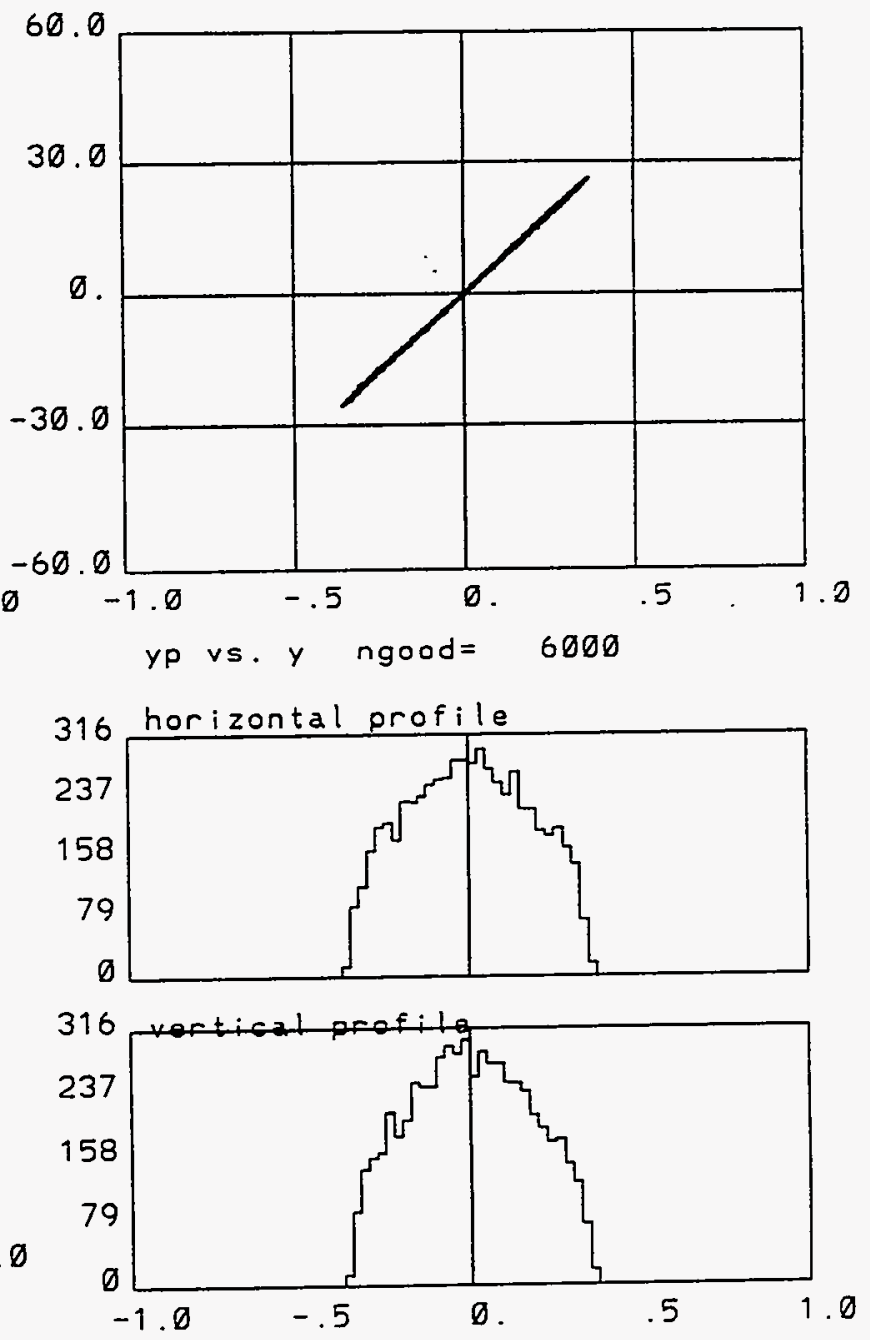
Figure 9 -5 shows the beam profile at position $(n e=5) z=10$, with the Inline-Injection system of Solenoid + Gun + Solenoid combination inline with the linac. With Solenoid current of $\mathrm{I}=2600 \mathrm{amp}$.

- BNL RF GUN

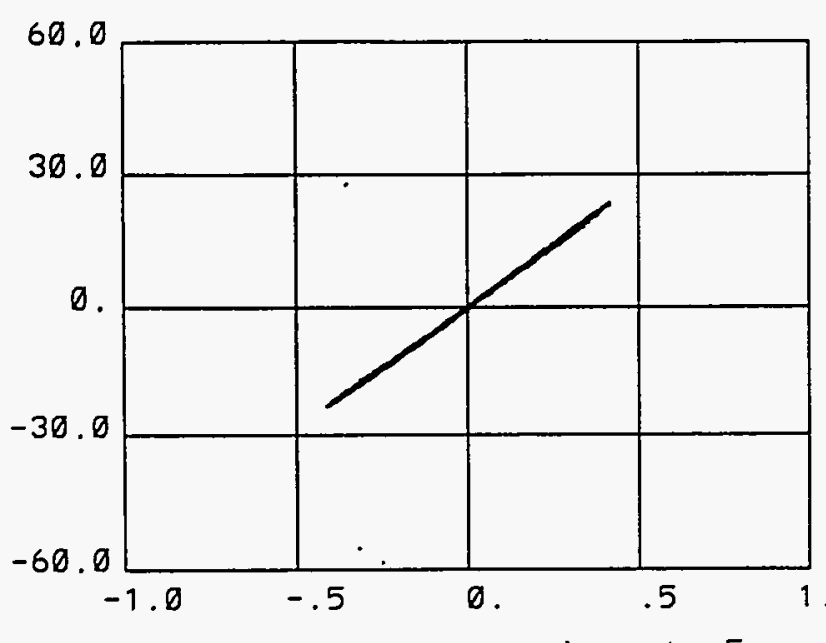

$x p \vee s . x$

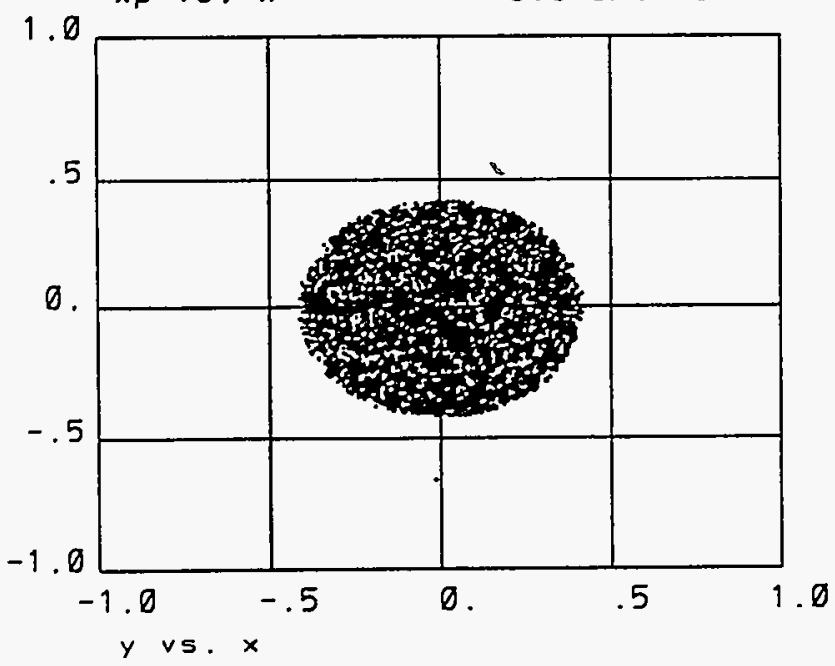

15.15ps $G(10), 1 \mathrm{nC}$, phi $=43.)^{\circ}$

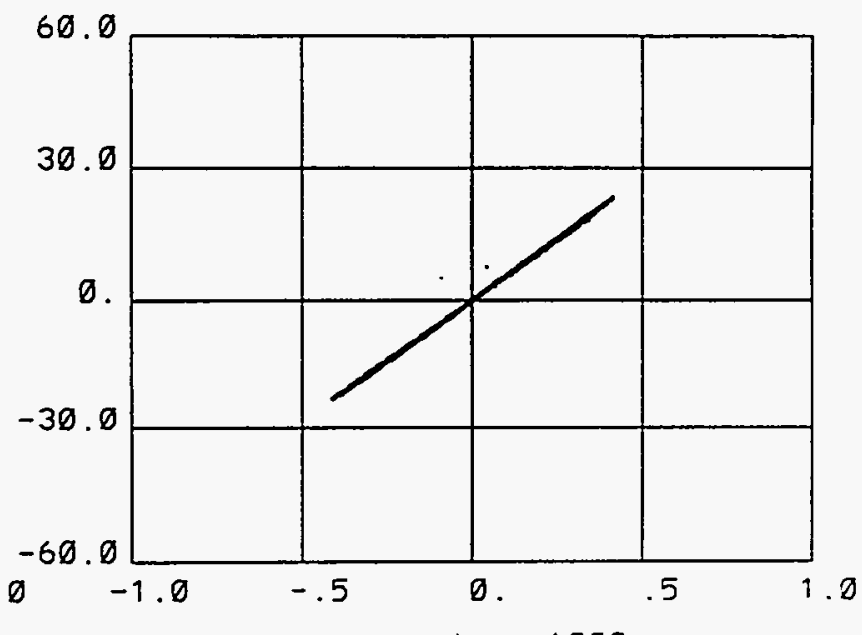

yp vs. y ngood $=6000$

300 horizontal profile
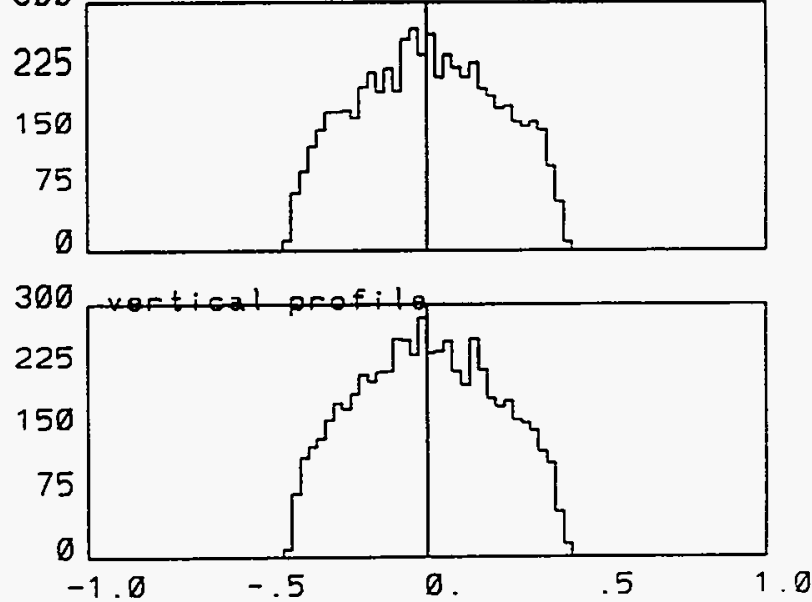
Figure 9-6 shows the beam profile at position $(n e=10) \quad z=20$, with the Inline-Injection system of Solenoid + Gun + Solenoid combination inline with the linac. With Solenoid current of $\mathrm{I}=2600 \mathrm{amp}$.

-BNL RF GUN
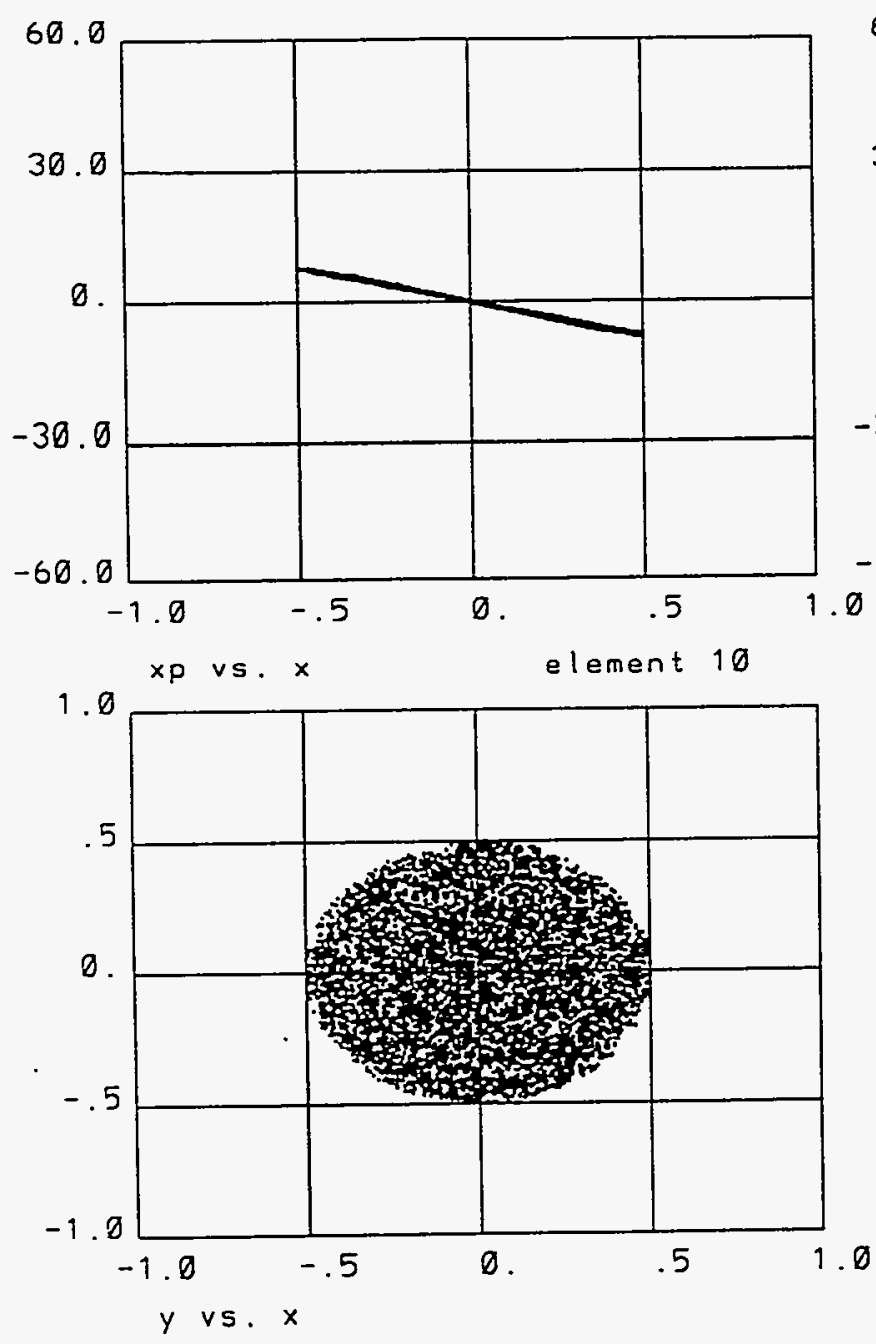

15.15ps G(10),1 nC, phi $=43.1^{\circ}$
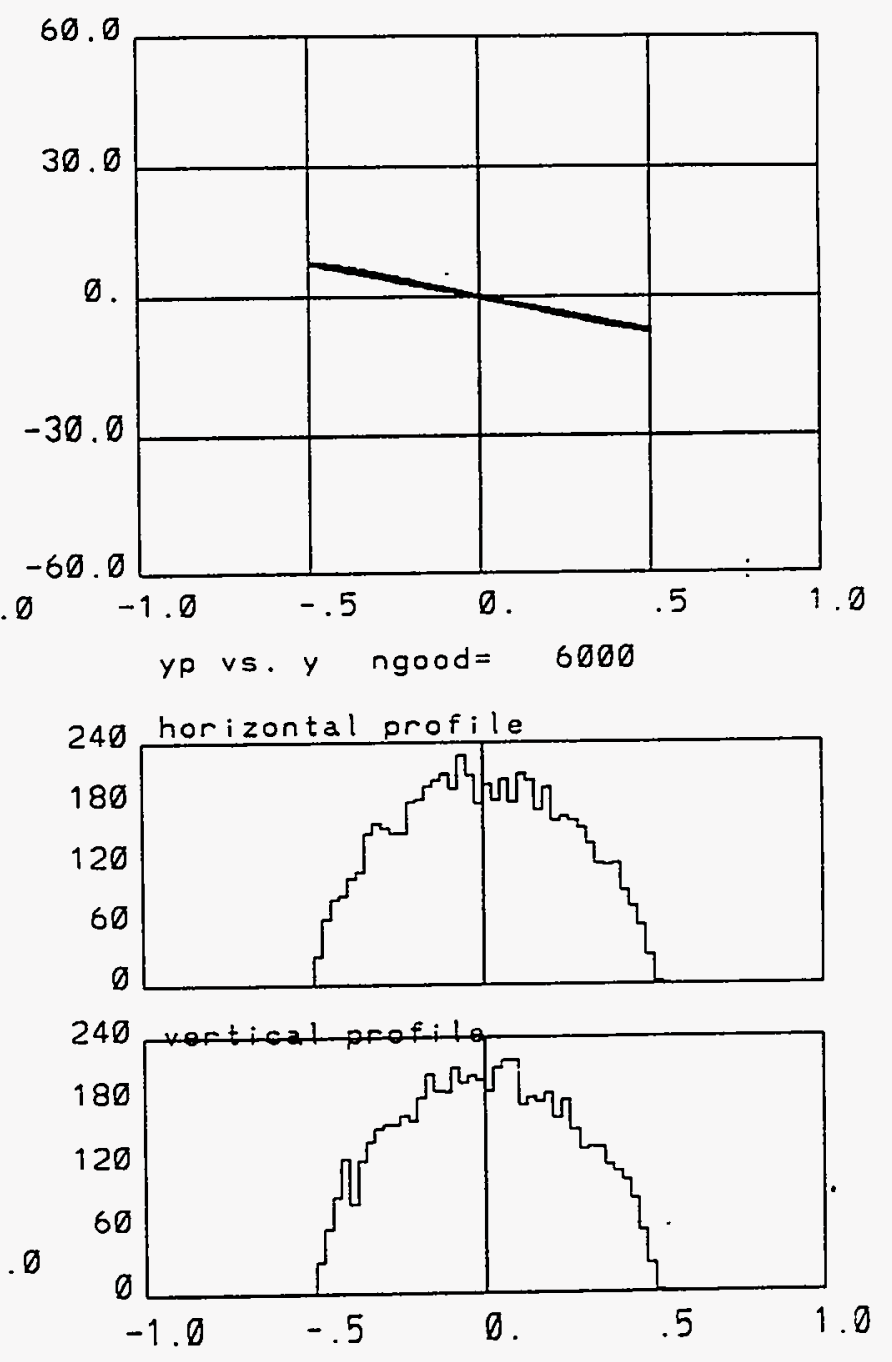
Figure $9-7$ shows the beam profile at position $(n e=20) z=40$, with the Inline-Injection system of Solenoid + Gun + Solenoid combination inline with the linac. With Solenoid current of $I=2600$ amp.

- BNL RF GUN
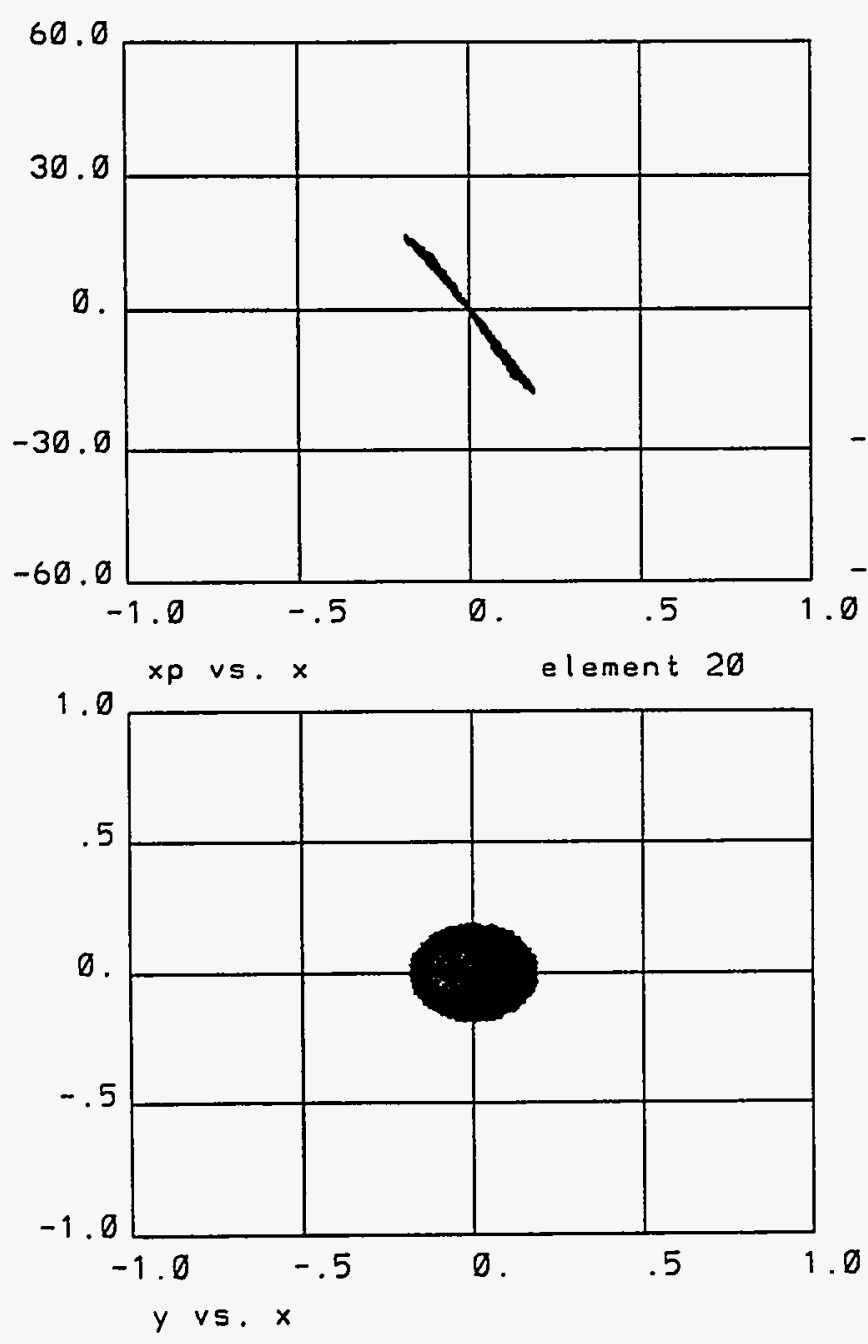

15.15ps $G(10), 1 \mathrm{nC}$, phi=43.1.

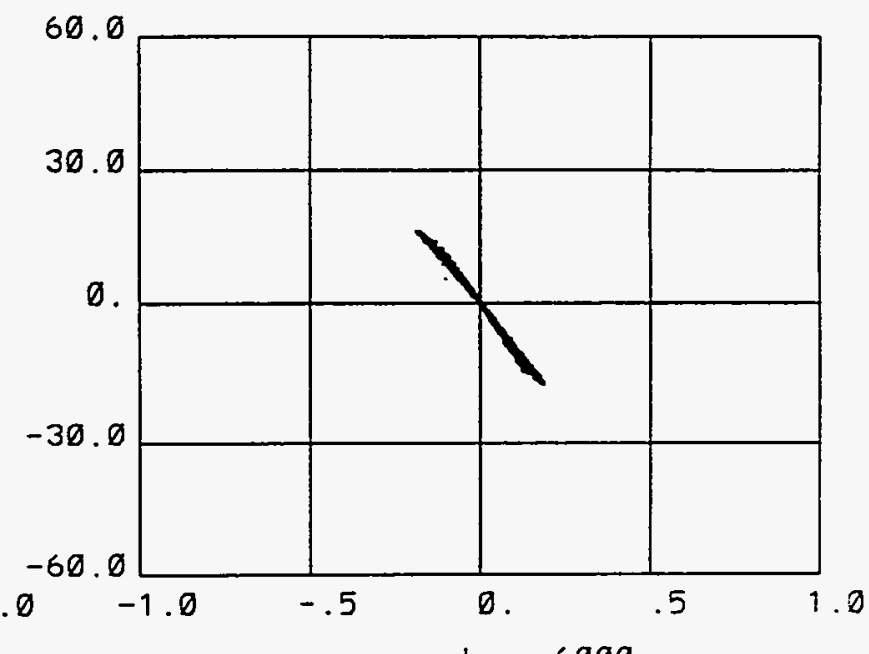

yp vs. y ngood $=6000$
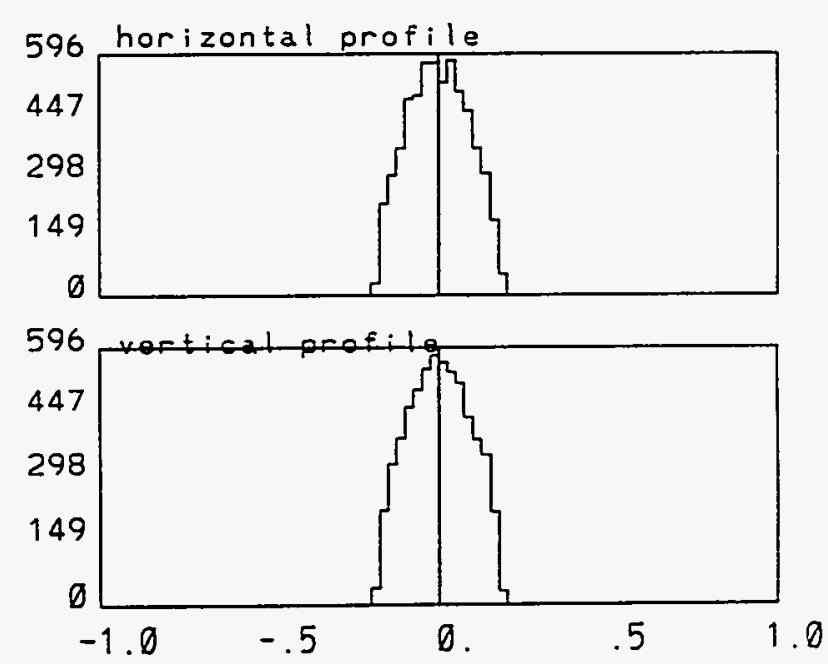
Figure 9-8 shows the beam profile at position $(n e=30) z=70$, with the Inline-Injection system of Solenoid + Gun + Solenoid combination inline with the linac. With Solenoid current of $I=2600$ amp.

'BNL RF GUN
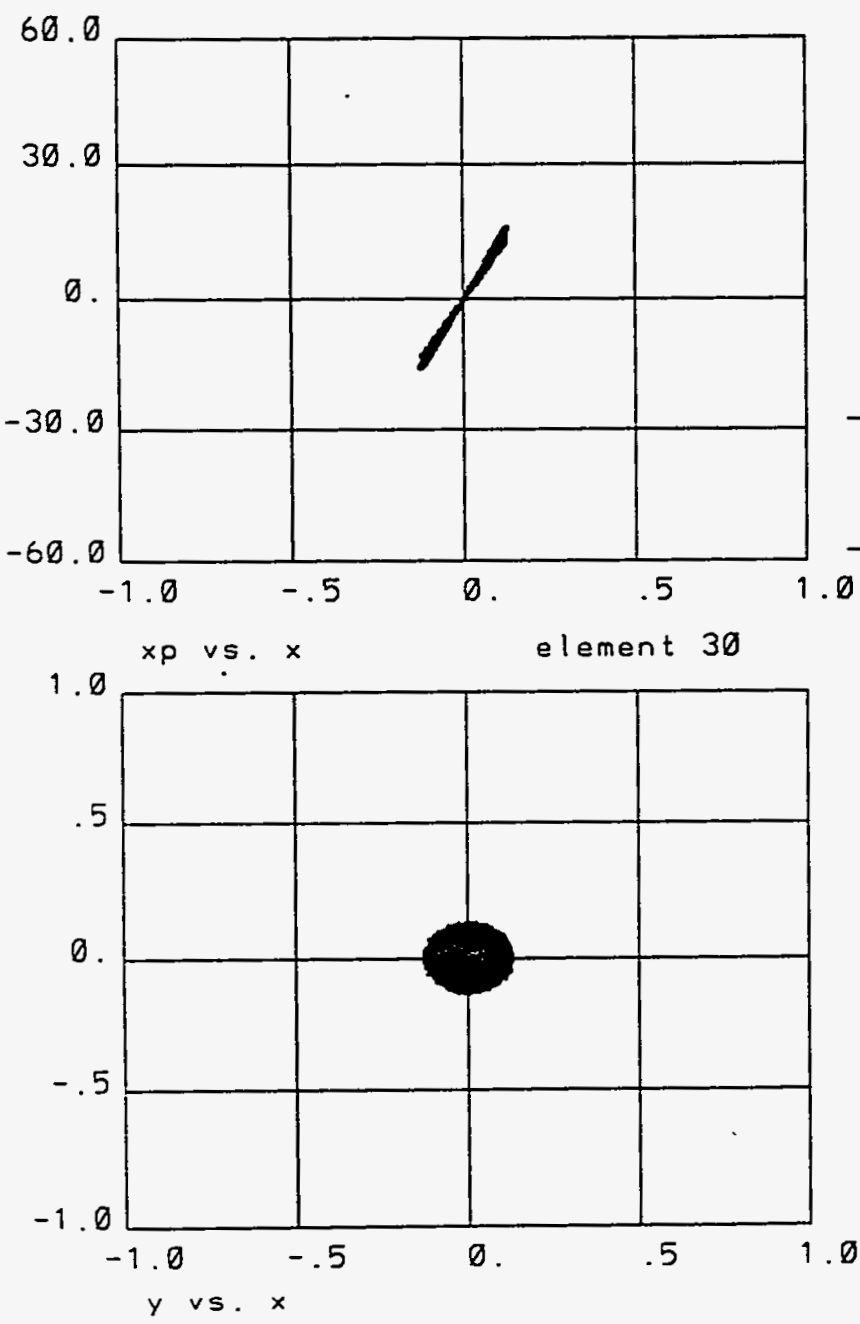

, $5.15 p s$ G(10), 1 nC, phi $=43.1^{\circ}$
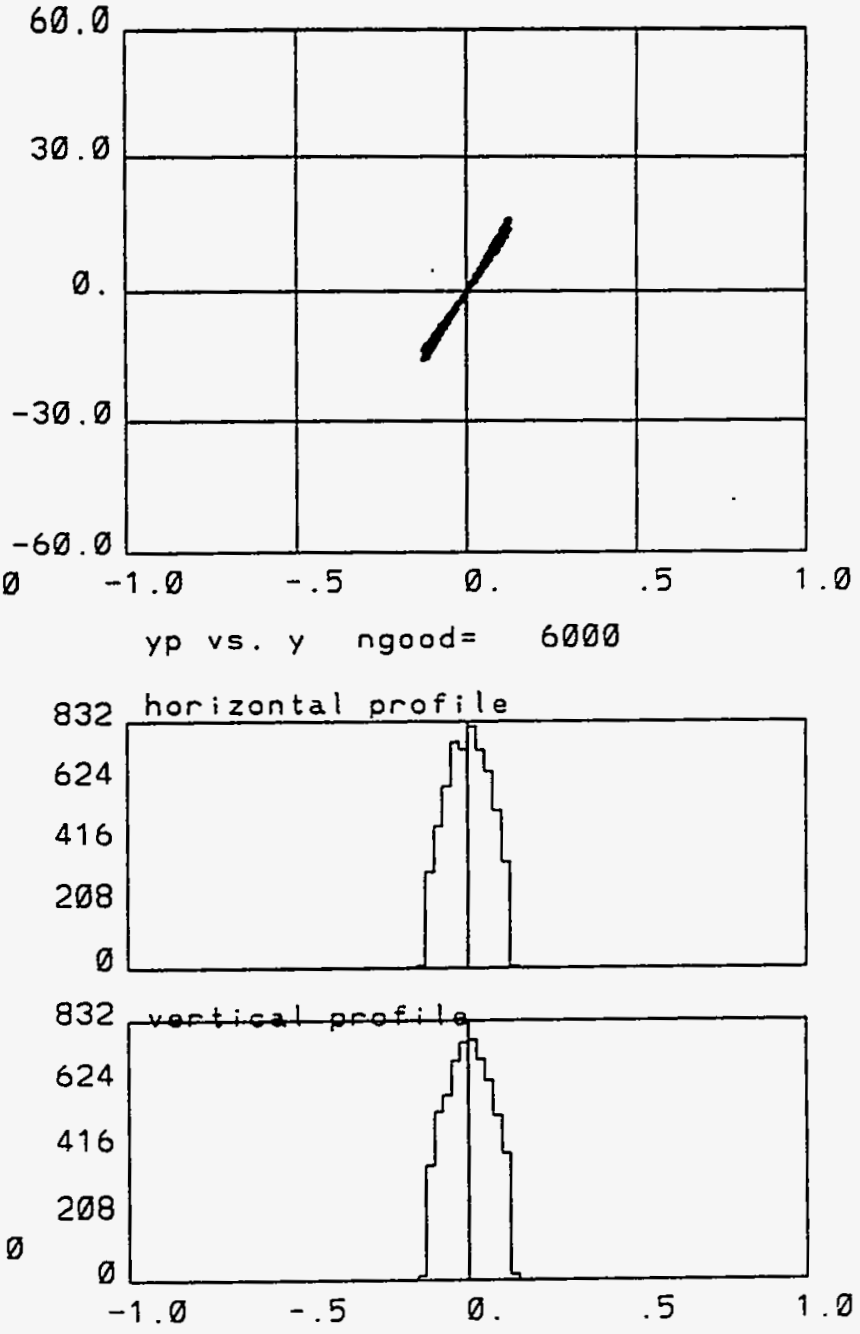
Figure 9-9 shows the beam profile at position (ne $=34) \quad z=90$, with the Inline-Injection system of Solenoid + Gun + Solenoid combination inline with the linac. With Solenoid current of $I=2600 \mathrm{amp}$.

'BNL RF GUN

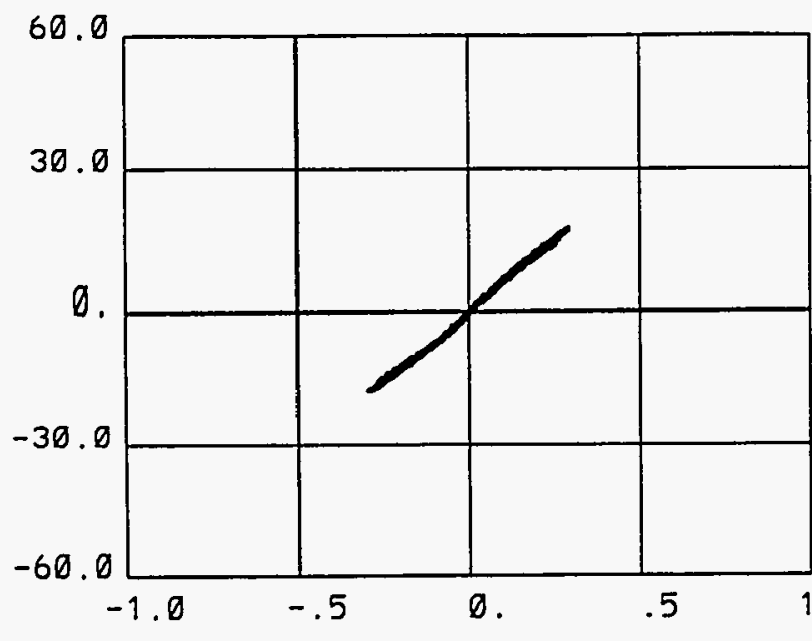

xp vs. $x$ element 34

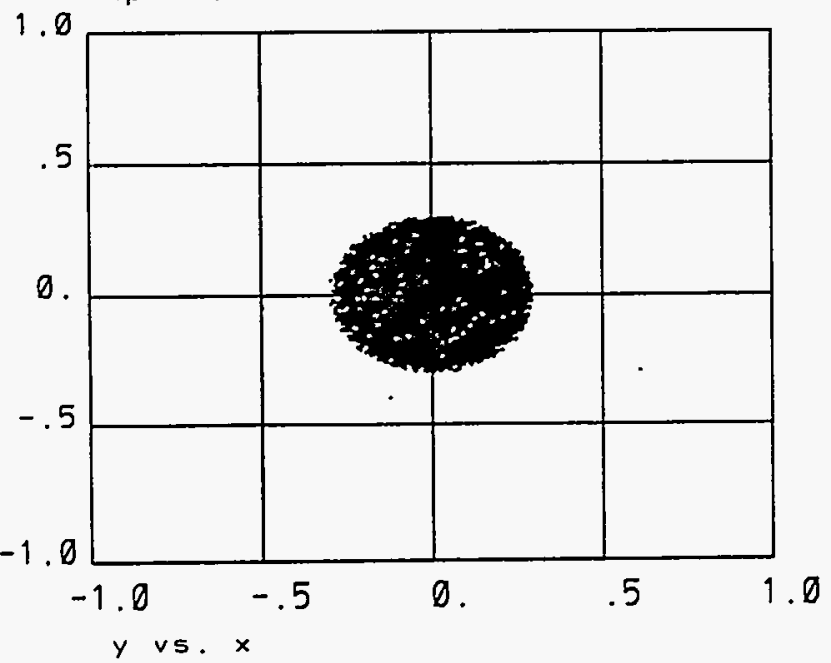

$5.15 p s G(10), 1 \mathrm{nC}$, phi $=43.)^{\text {. }}$

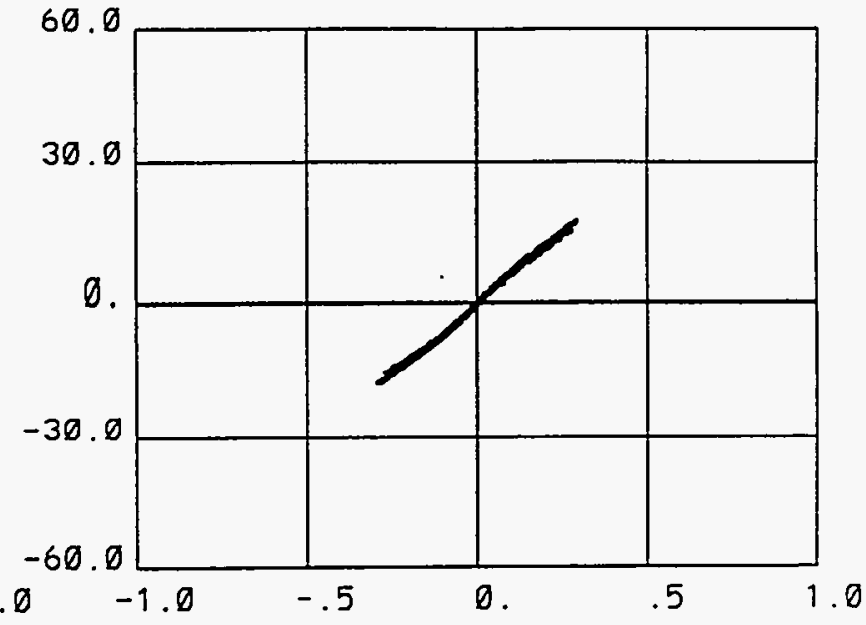

yp vs. y ngood $=6000$
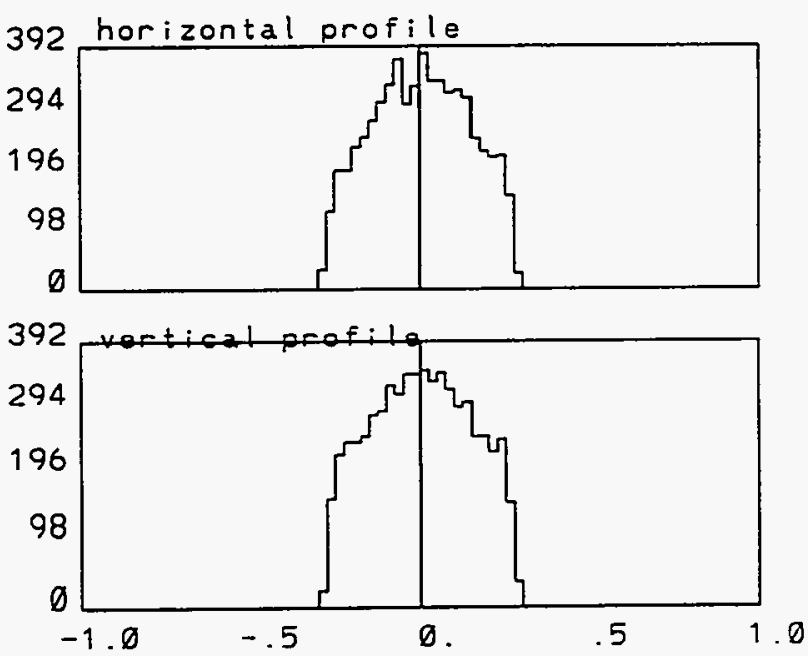
Figures 10-1 to 10-9 shows the beam profile at various positions istance $\mathbf{z}$ from the cathode), with the Inline-Injection system of Solenoid + Gun + Solenoid combination inline with the linac. With Solenoid current of I $=2800 \mathrm{amp}$.

-BNL RF GUN

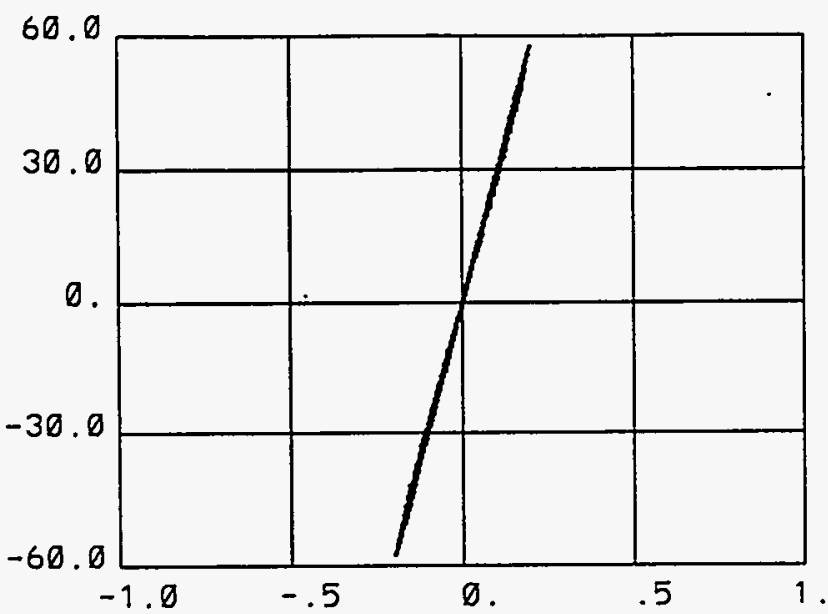

$-1.0-.5$

xp vs. $x$

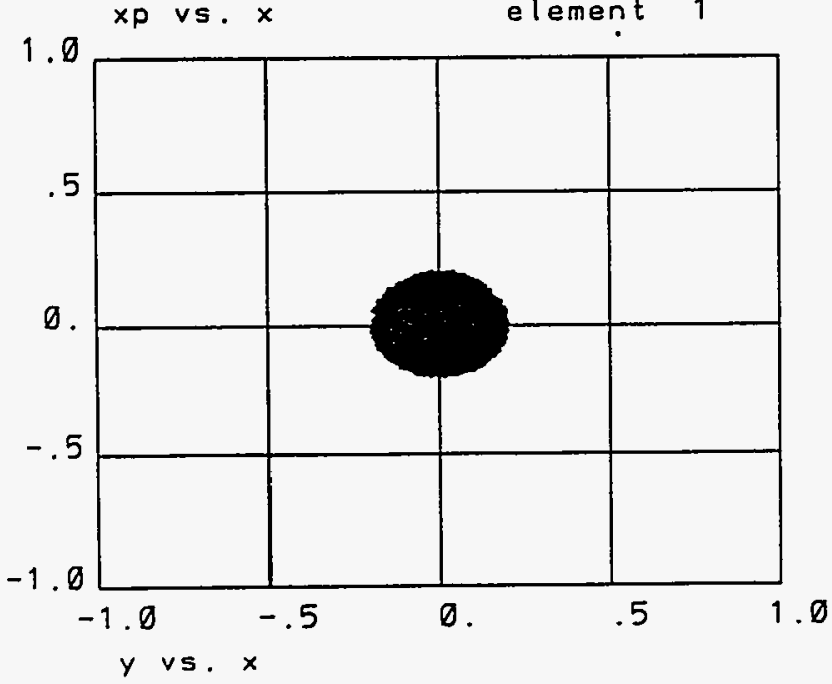

$15.15 p$ s $G(10), 1$ nC, phi=43.).

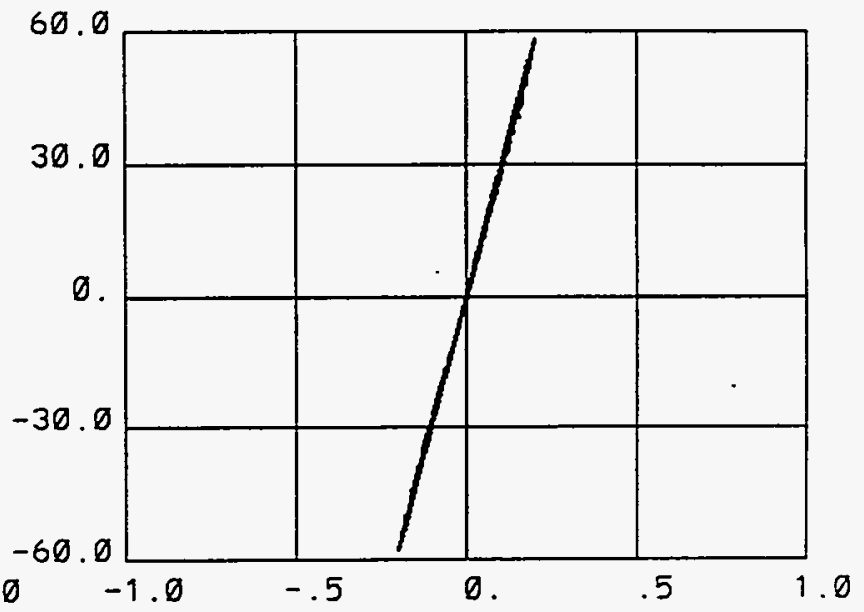

yp vs. y ngood $=6000$

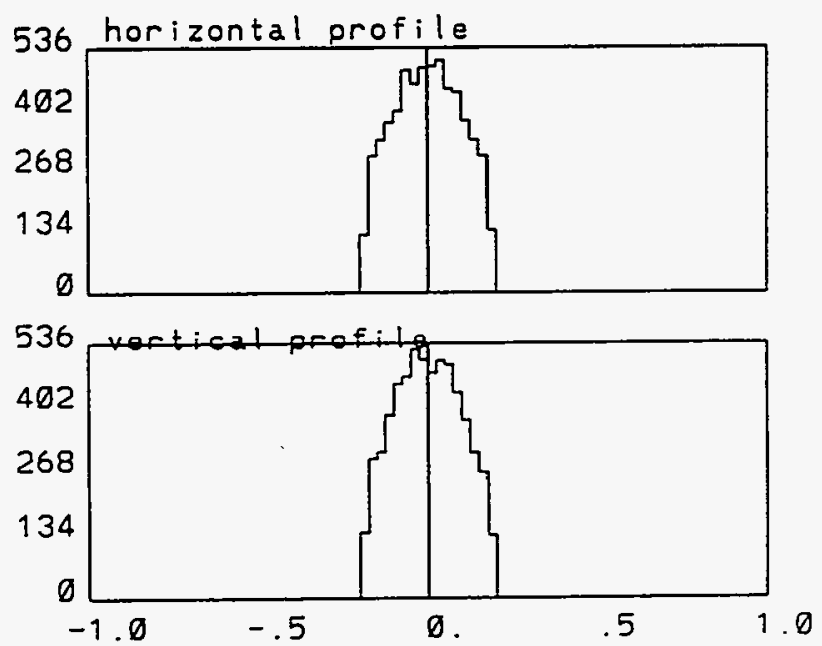

Figure $10-1$ shows the beam profile at position $(n e=1) z=0$, with the Inline-Injection system of Solenoid + Gun + Solenoid combination inline with the linac. With Solenoid current of $I=2800 \mathrm{amp}$. 
Figure $10-2$ shows the beam profile at position $(n e=2) z=2.6$, with the Inline-Injection system of Solenoid + Gun + Solenoid combination inline with the linac. With Solenoid current of $I=2800 \mathrm{amp}$.

- BNL RF GUN

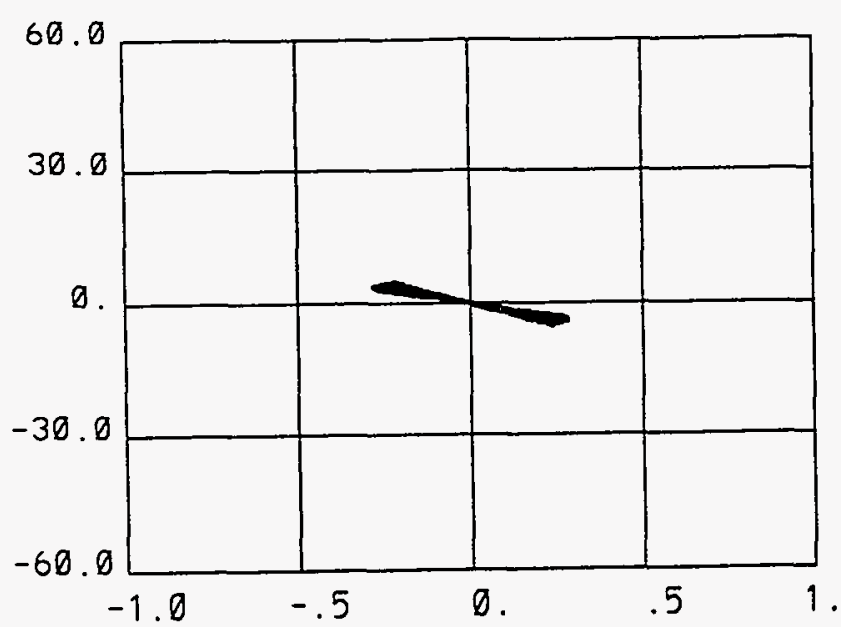

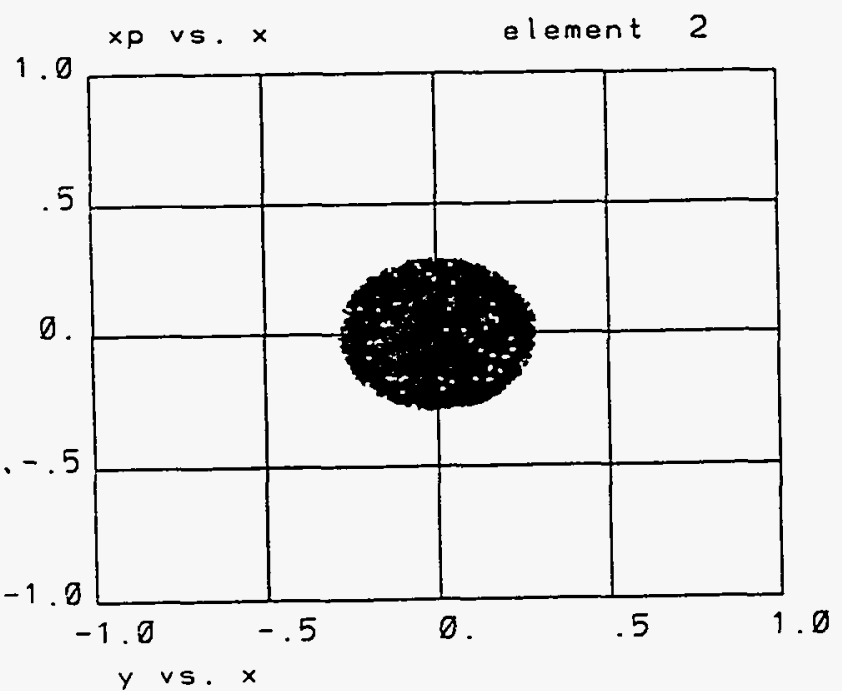

$.5 .15 p s G(10), 1$ nC, ohi $=43.)^{\circ}$

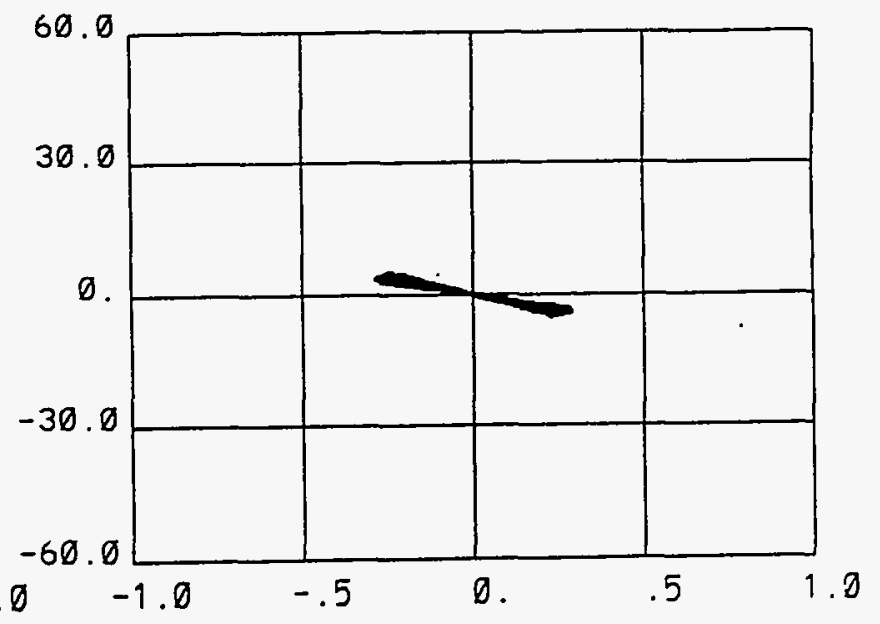

yp vs. y ngood $=6000$
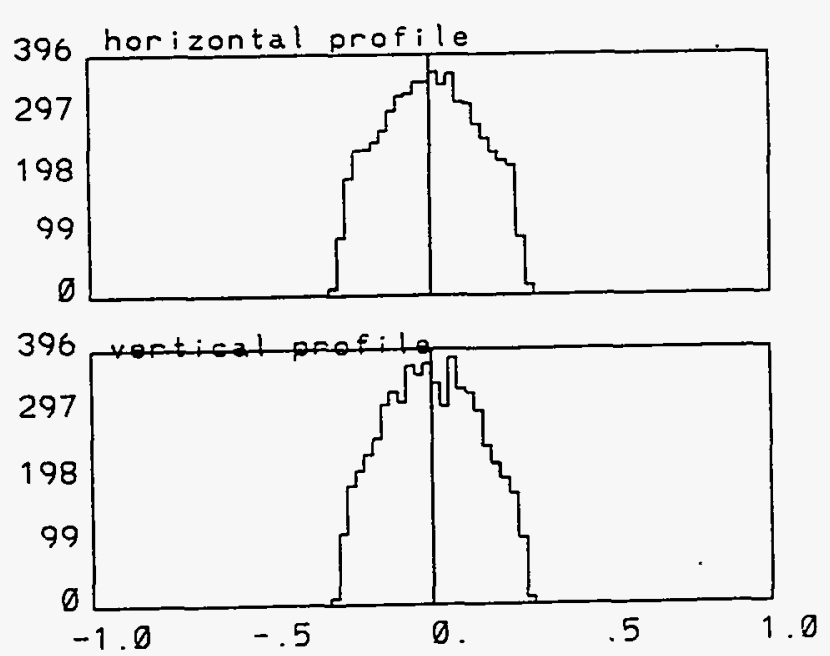
Figure $10-3$ shows the beam profile at position $(n e=3) z=5.2$, with the Inline-Injection system of Solenoid + Gun + Solenoid combination inline with the linac. With Solenoid current of $\mathrm{I}=2800 \mathrm{amp}$.

- BNL RF GUN

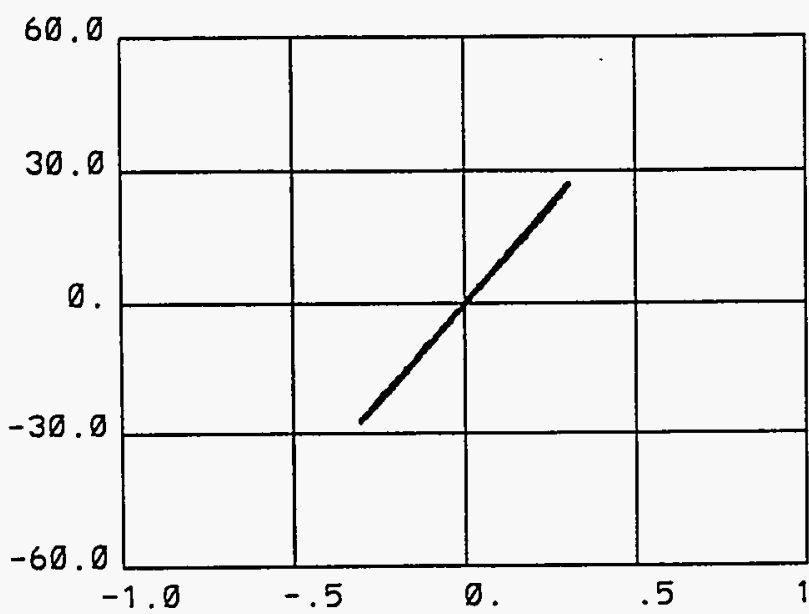

$x p$ vs. $x$

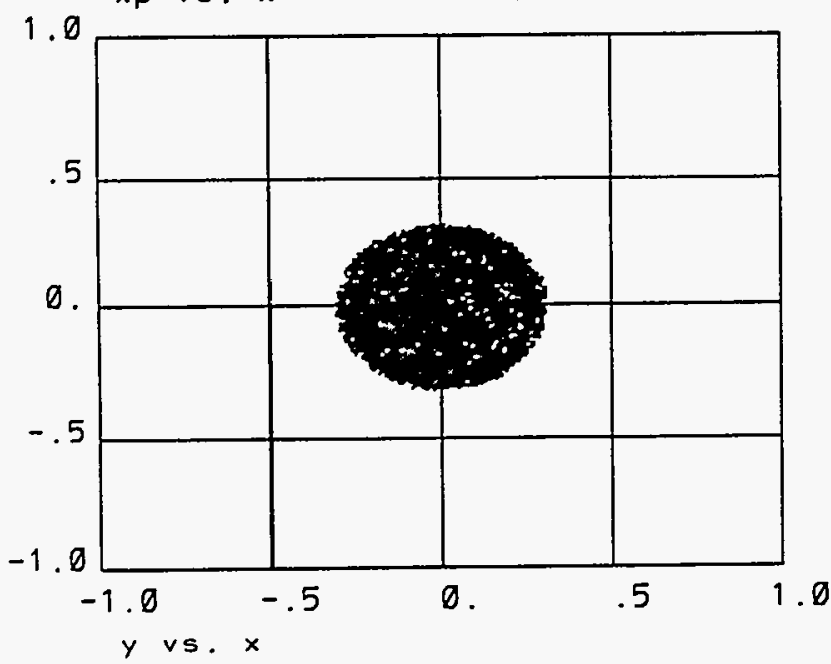

,5.15ps $G(10), 1$ nC, phi=43.).

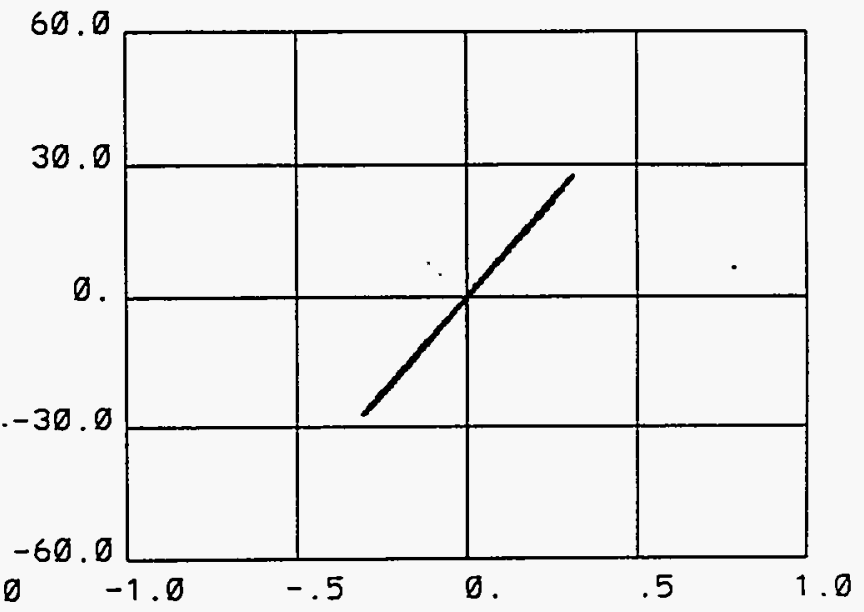

yp vs. y ngood $=6000$

376 horizontal profile
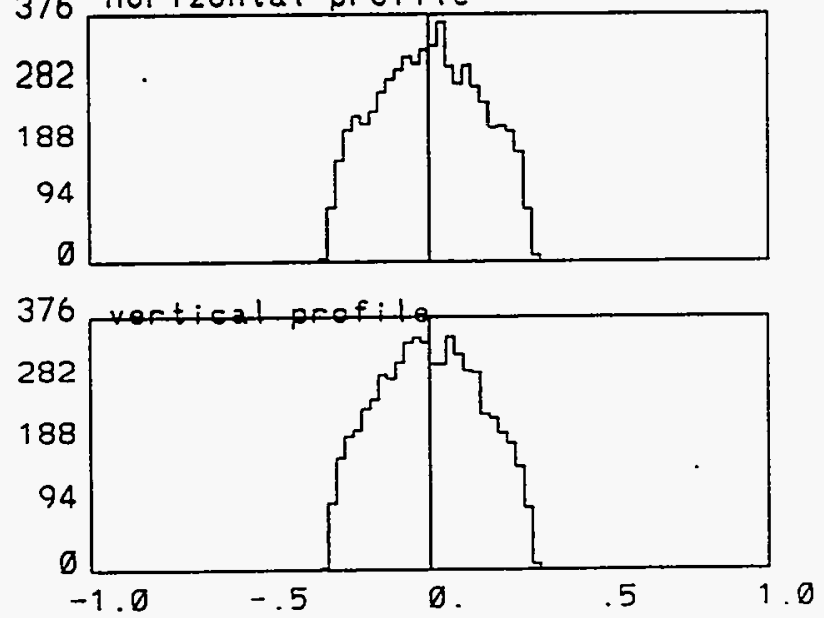
Figure 10-4 shows the beam profile at position $(n e=4) z=7.9$, with the Inline-Injection system of Solenoid + Gun + Solenoid combination inline with the linac. With Solenoid current of $\mathrm{I}=\mathbf{2 8 0 0} \mathrm{amp}$.

- BNL RF GUN
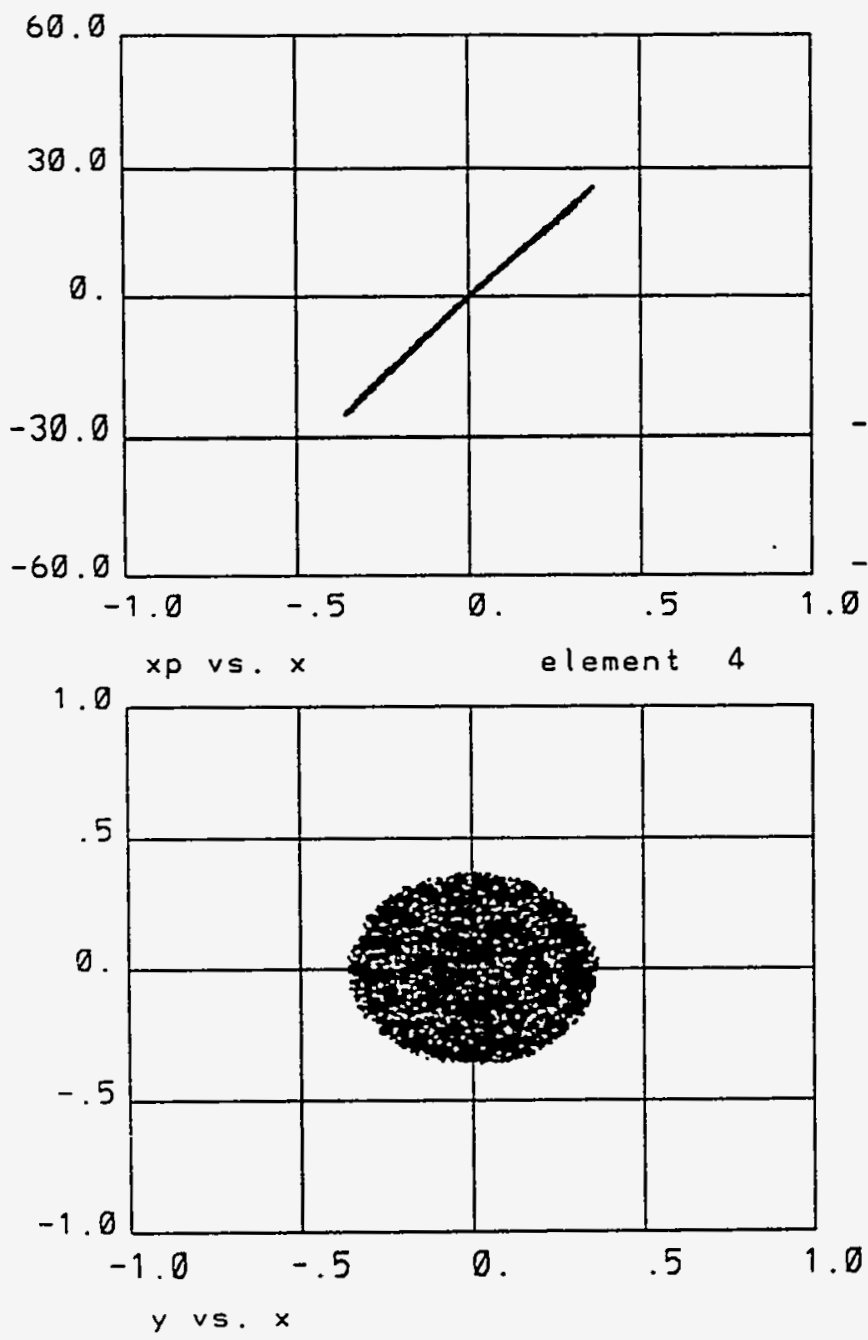

,5.15ps $G(10), 1 \mathrm{nC}$, phi $=43.1^{\circ}$
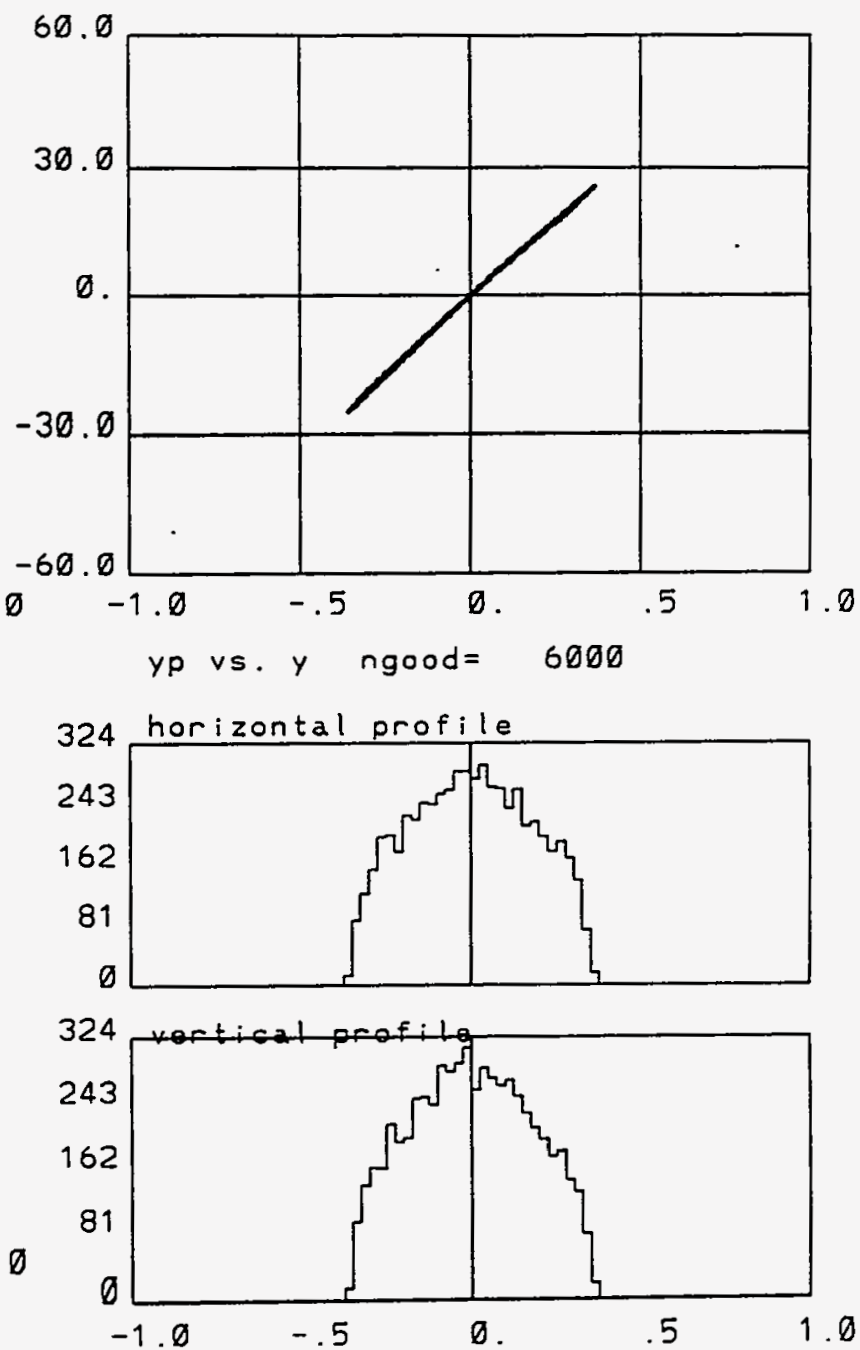
Figure $10-5$ shows the beam profile at position $(n e=5) \quad z=10$, with the Inline-Injection system of Solenoid + Gun + Solenoid combination inline with the linac. With Solenoid current of $I=2800$ amp.

- BNL RF GUN
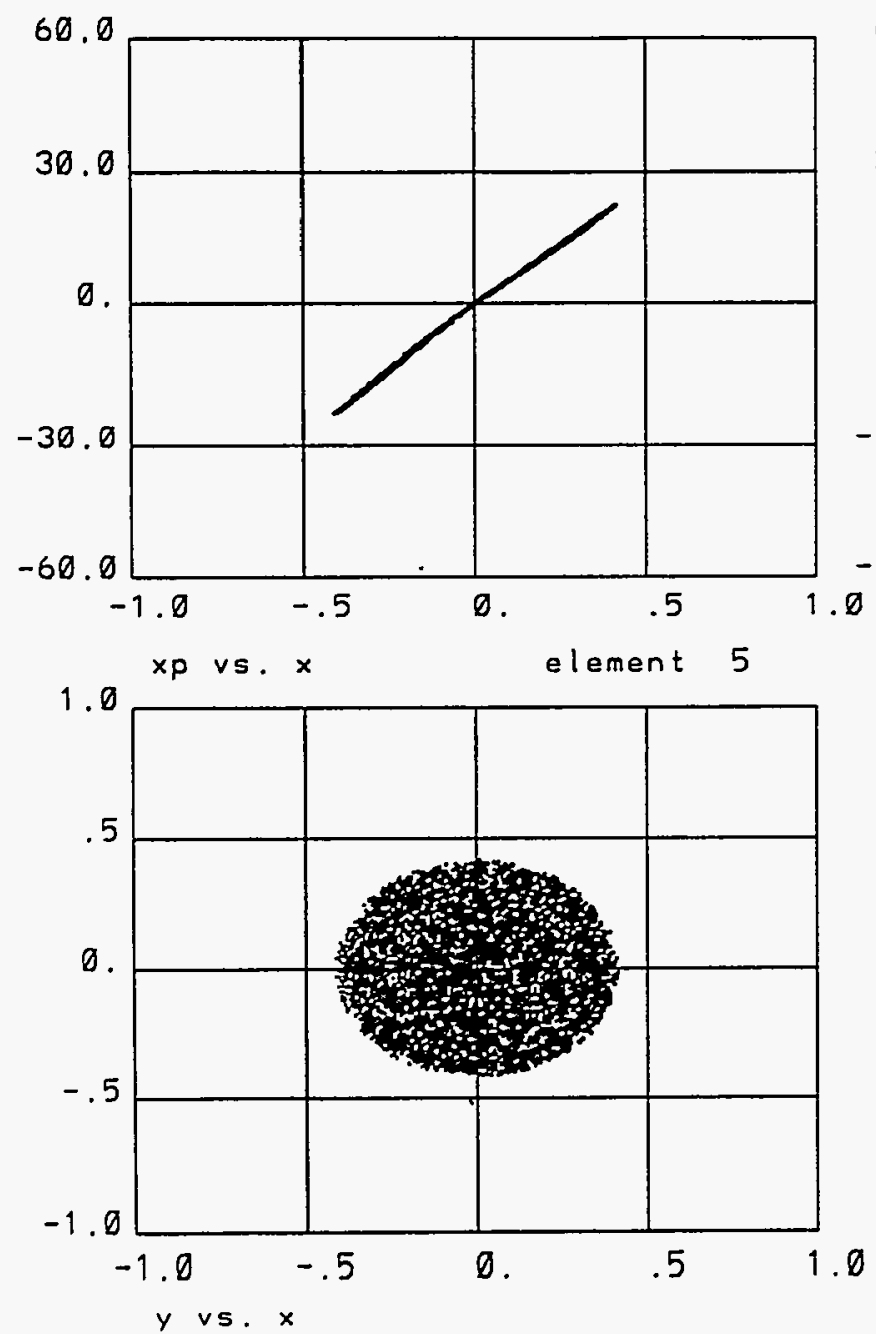

$, 5.15 p s \mathrm{G}(10), 1 \mathrm{nC}, \mathrm{phi}=43.)^{\text {. }}$

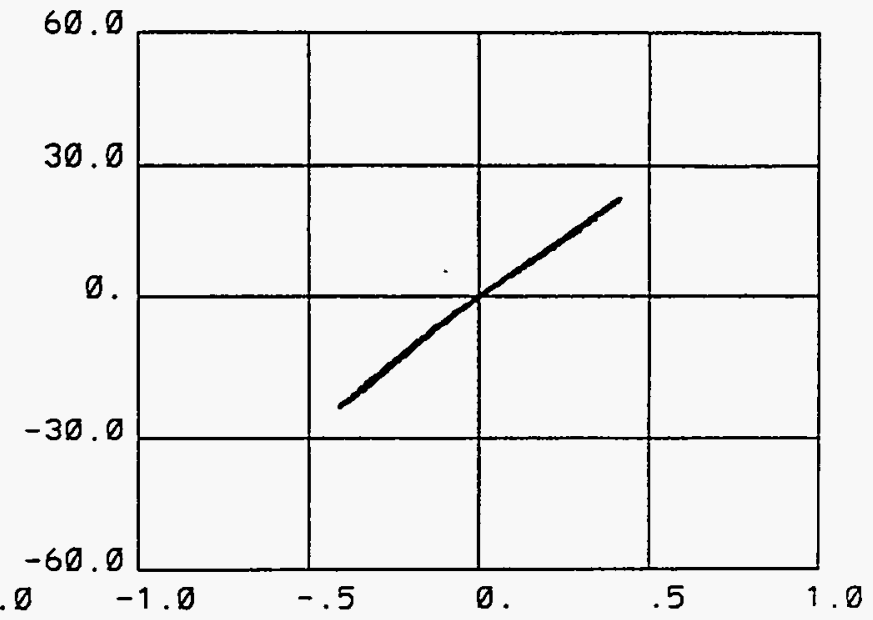

yp vs. y ngood $=6000$
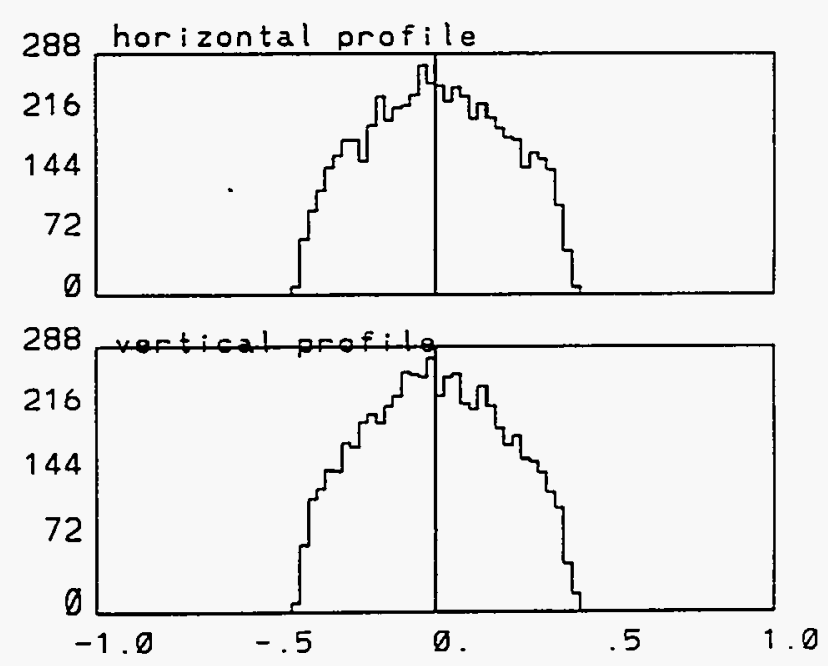
Figure $10-6$ shows the beam profile at position $(n e=10) \quad z=20$, with the Inline-Injection system of Solenoid + Gun + Solenoid combination inline with the linac. With Solenoid current of $\mathrm{I}=2800$ amp.

-BNL RF GUN
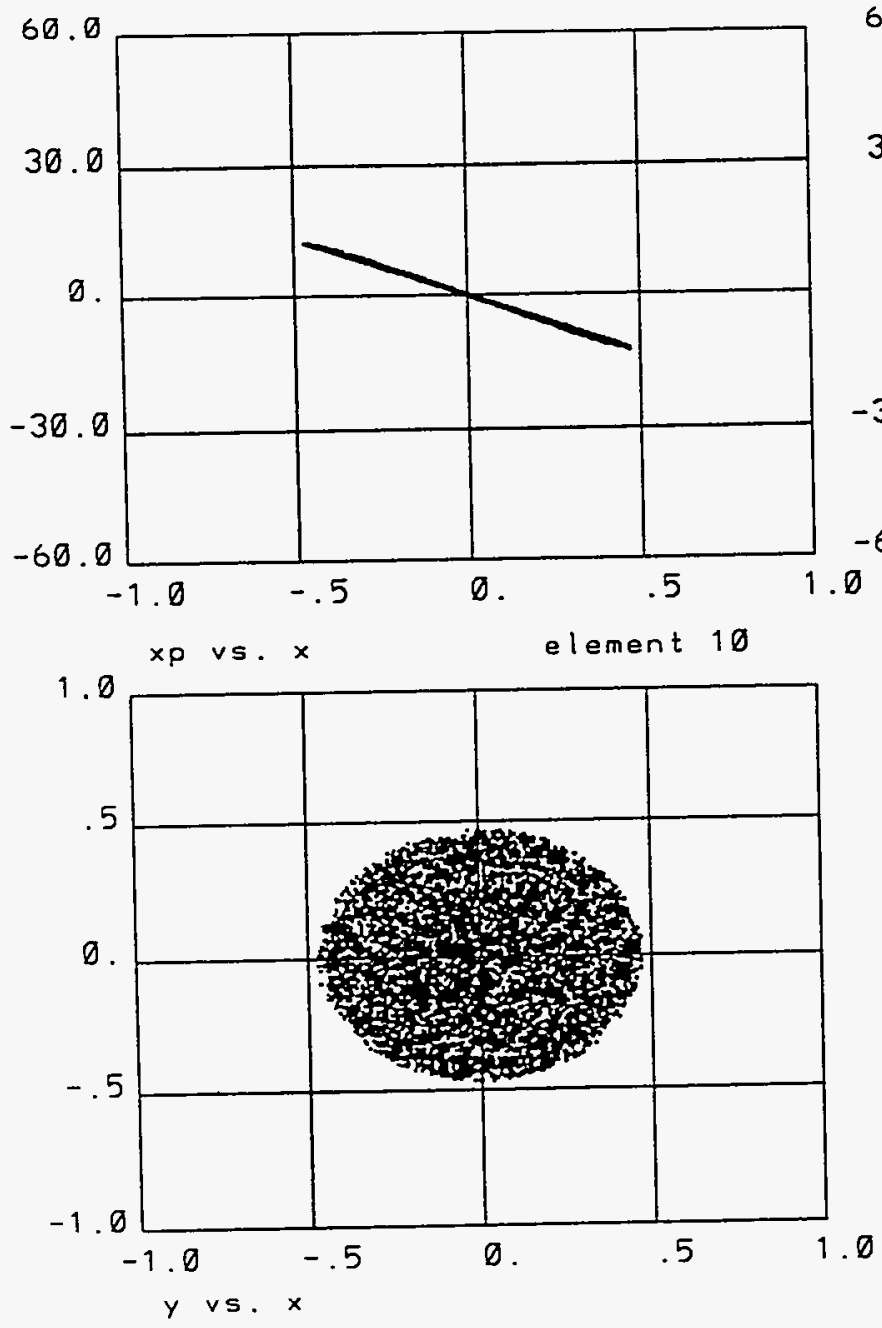

$15.15 p s$ G(10),1 nC, phi $=43.1^{\circ}$
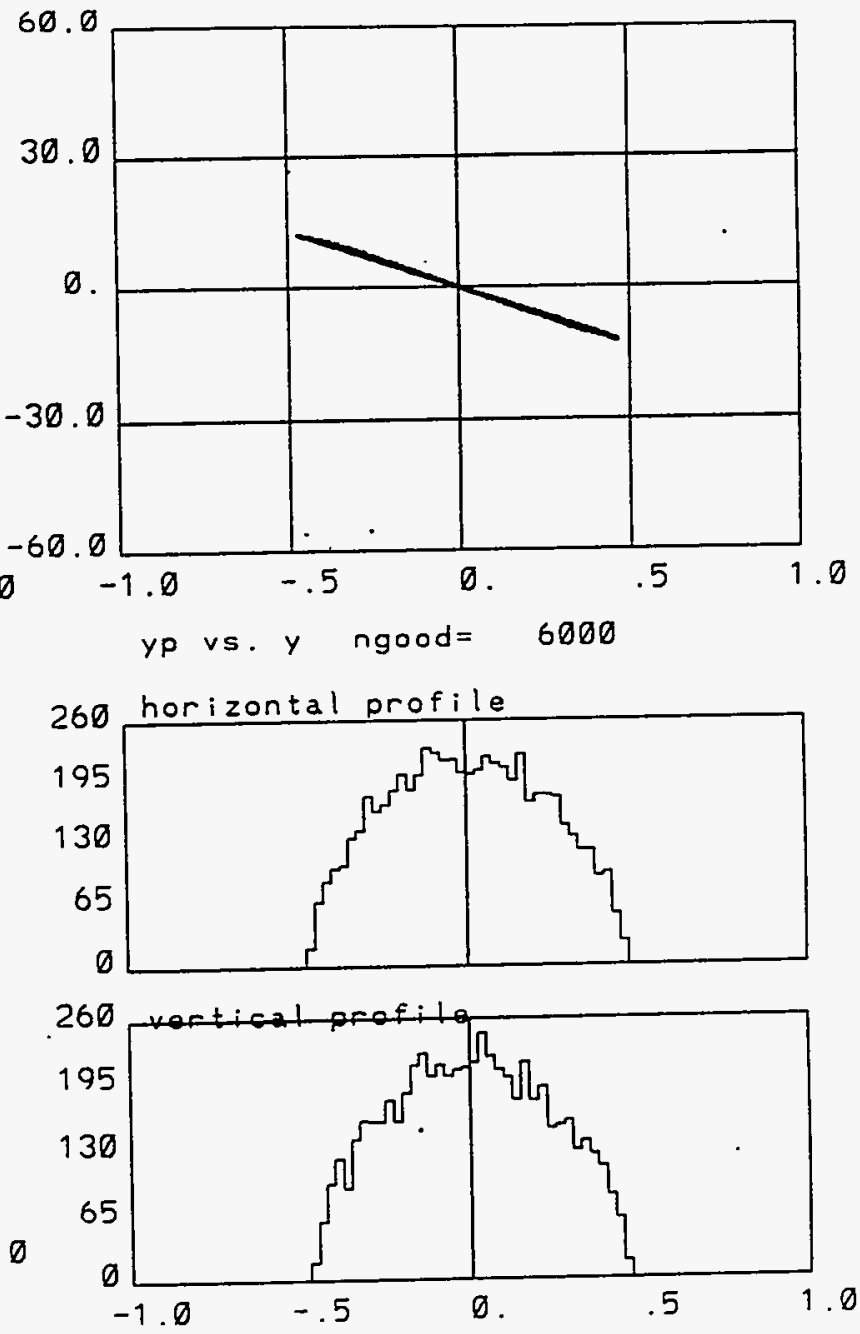
Figure $10-7$ shows the beam profile at position $(n e=20) z=40$, with the Inline-Injection system of Solenoid + Gun + Solenoid combination inline with the linac. With Solenoid current of $\mathrm{I}=\mathbf{2 8 0 0} \mathrm{amp}$.

- BNL RF GUN
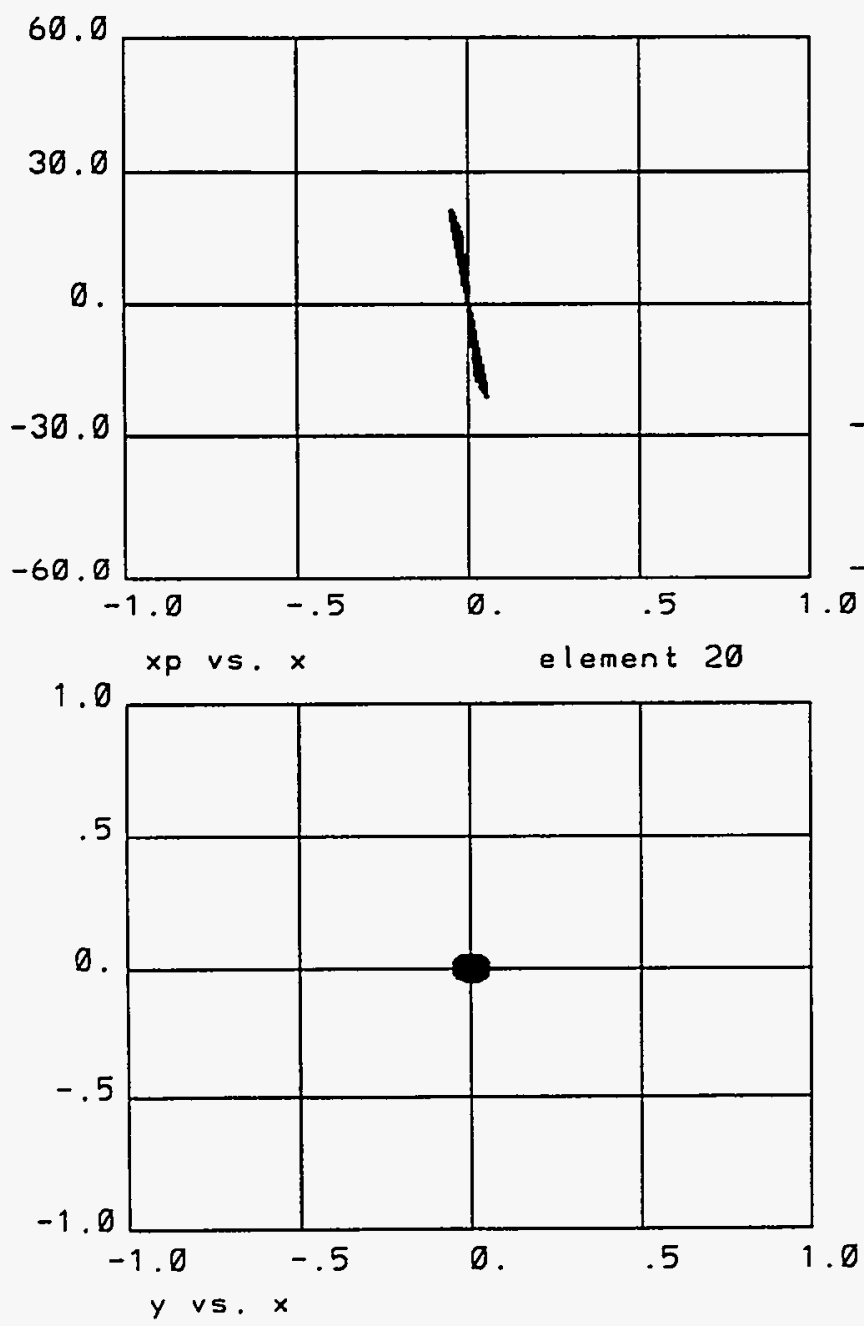

$, 5.15 p s \mathrm{G}(10), 1 n C$, phi $i=43.)^{\circ}$
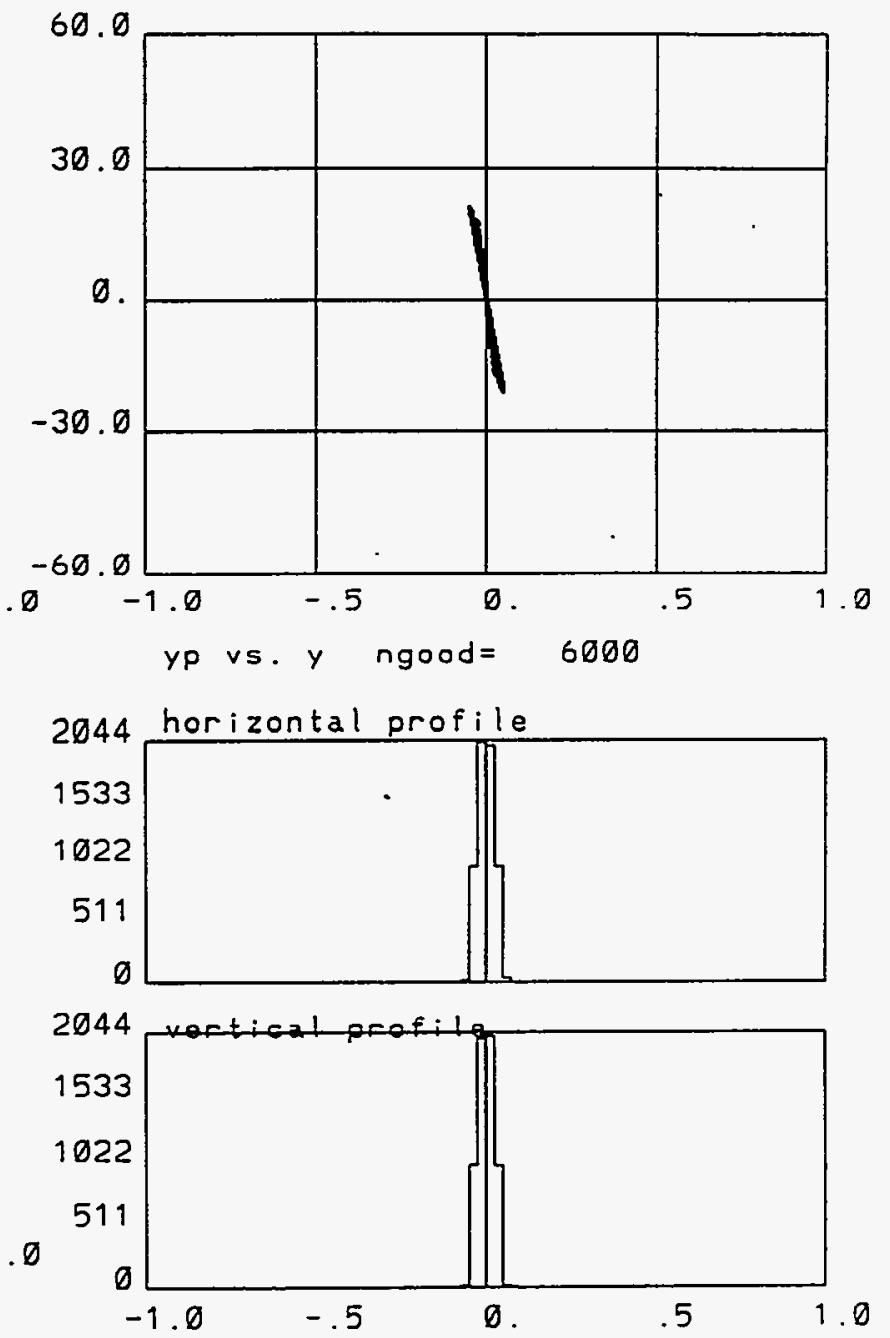
Figure 10-8 shows the beam profile at position $(n e=30) \quad z=70$, with the Inline-Injection system of Solenoid + Gun + Solenoid combination inline with the linac. With Solenoid current of $I=2800 \mathrm{amp}$.

- BNL RF GUN

60.0
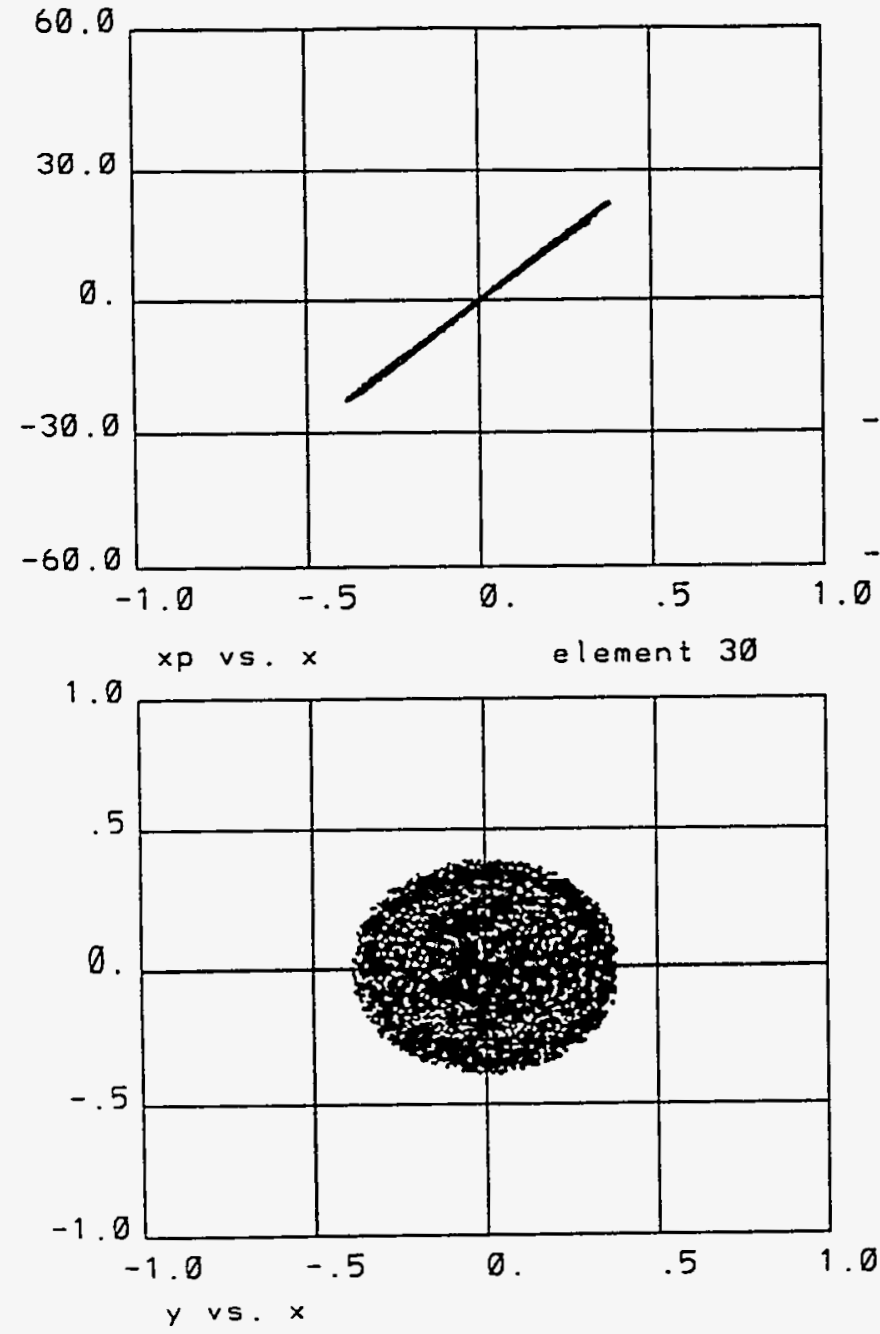

$.5 .15 p s \mathrm{G}(10), 1 \mathrm{nC}, \mathrm{ph} i=43.)^{\circ}$
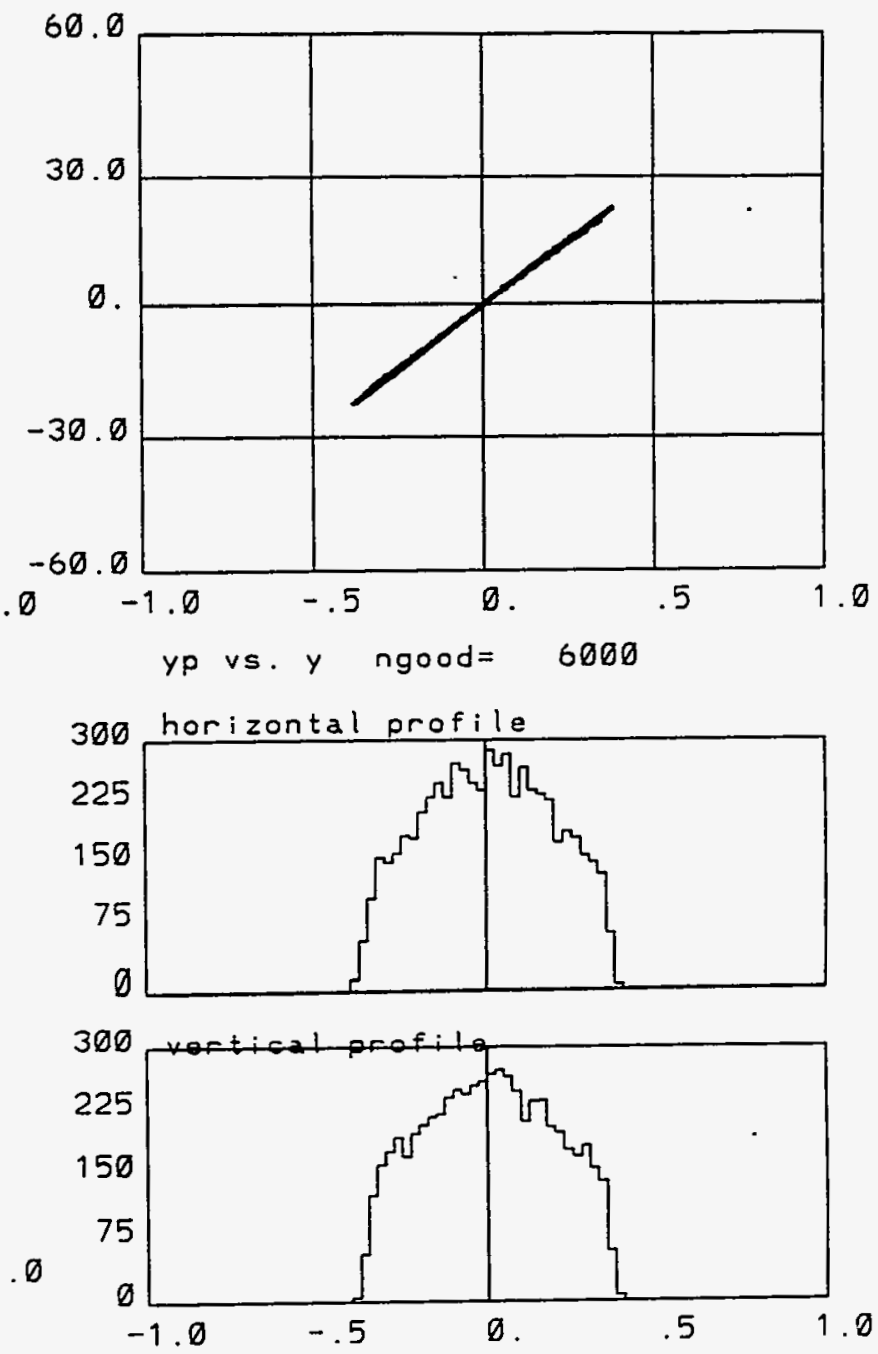
Figure 10-9 shows the beam profile at position $(n e=34) z=90$, with the Inline-Injection system of Solenoid + Gun + Solenoid combination inline with the linac. With Solenoid current of $I=2800 \mathrm{amp}$.

'BNL RF GUN

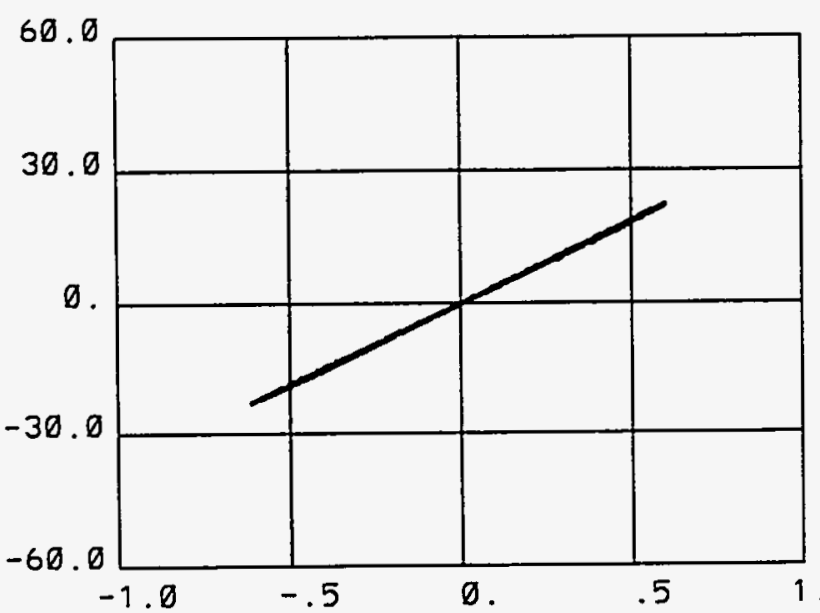

xp vs. $x$

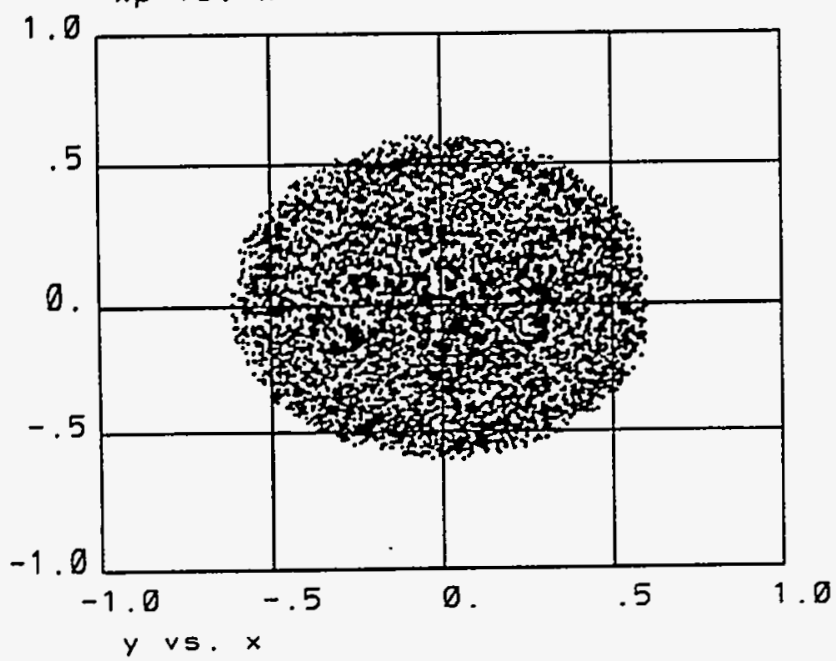

$1,5.15 p s$ Gl10),1 nC, phi $=43.1^{\circ}$

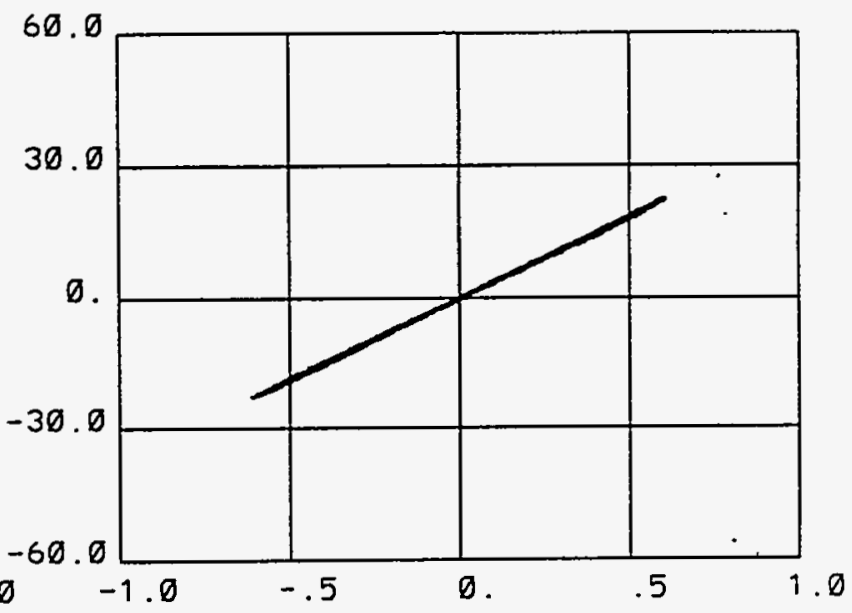

yp vs. y ngood $=6000$
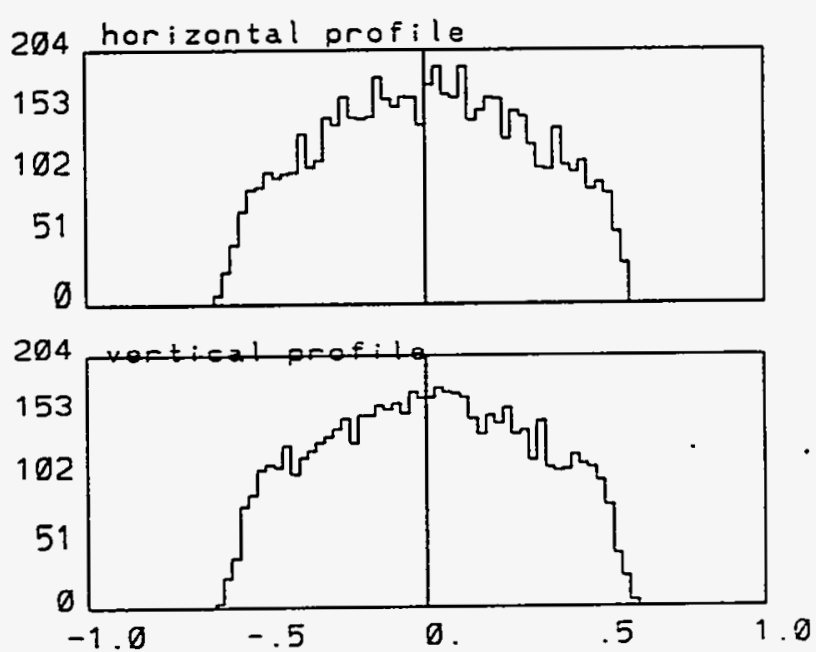
Figures $11-1$ to 11-9 shows the beam profile at various positions istance $z$ from the cathode), with the Inline-Injection system of Solenoid + Gun + Solenoid combination inline with the linac. With Solenoid current of I=3000 amp.

- BNL RF GUN
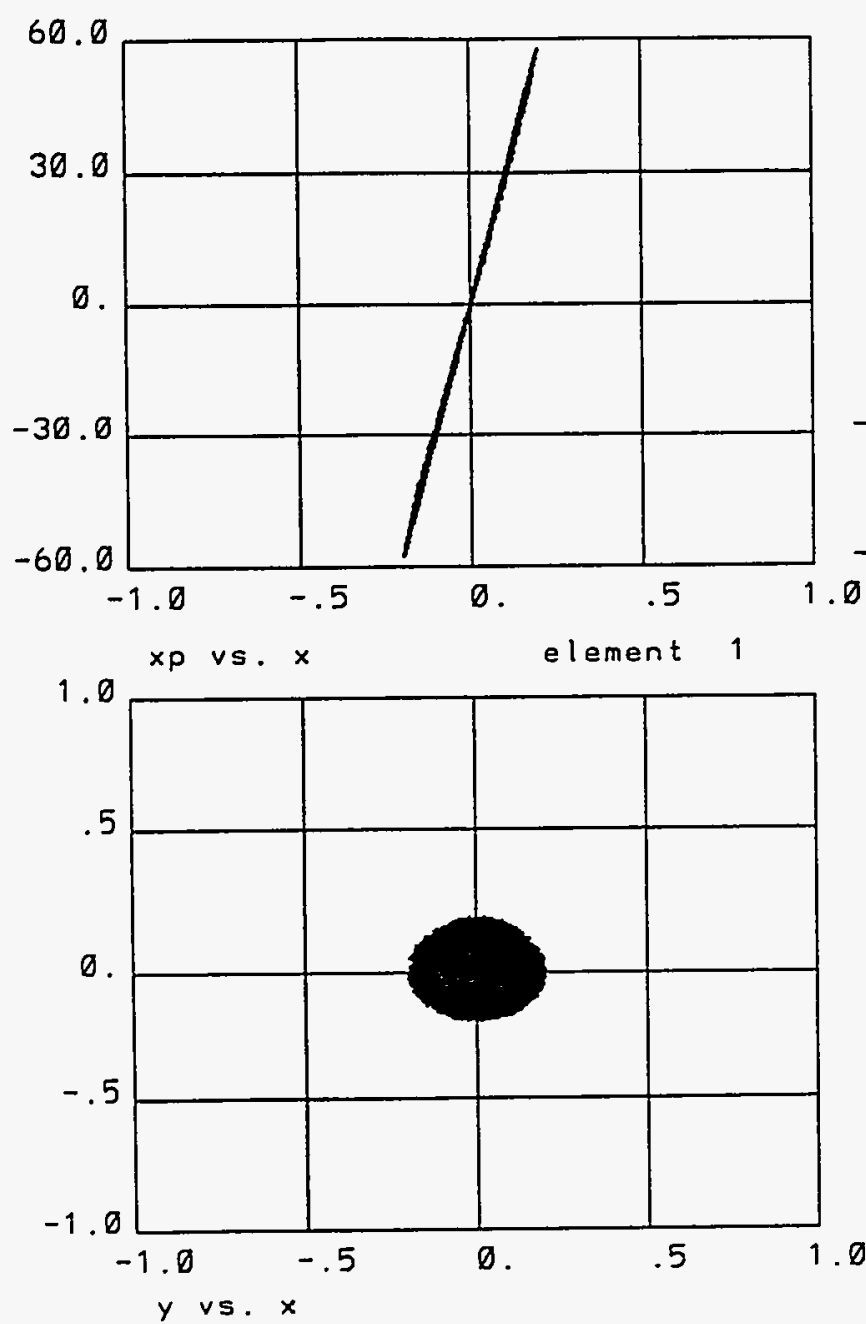

,5.15ps $G(10), 1$ nC, phi $=43.1$.
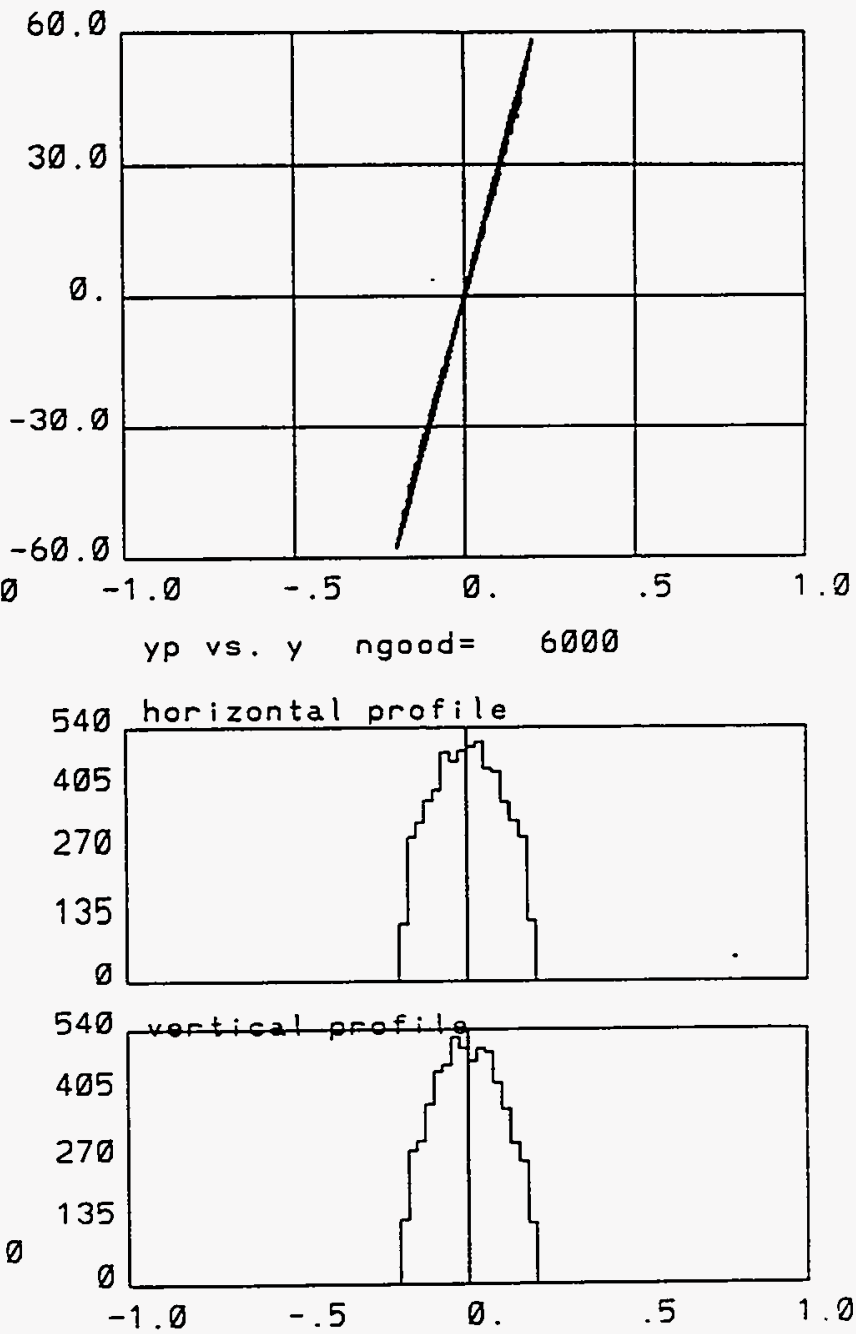

Figure $11-1$ shows the beam profile at position $(n e=1) \quad z=0$, with the Inline-Injection system of Solenoid + Gun + Solenoid combination inline with the linac. With Solenoid current of $I=3000$ amp. 
Figure $11-2$ shows the beam profile at position $(n e=2) z=2.6$, with the Inline-Injection system of Solenoid + Gun + Solenoid combination inline with the linac. With Solenoid current of $I=3000 \mathrm{amp}$.

- BNL RF GUN

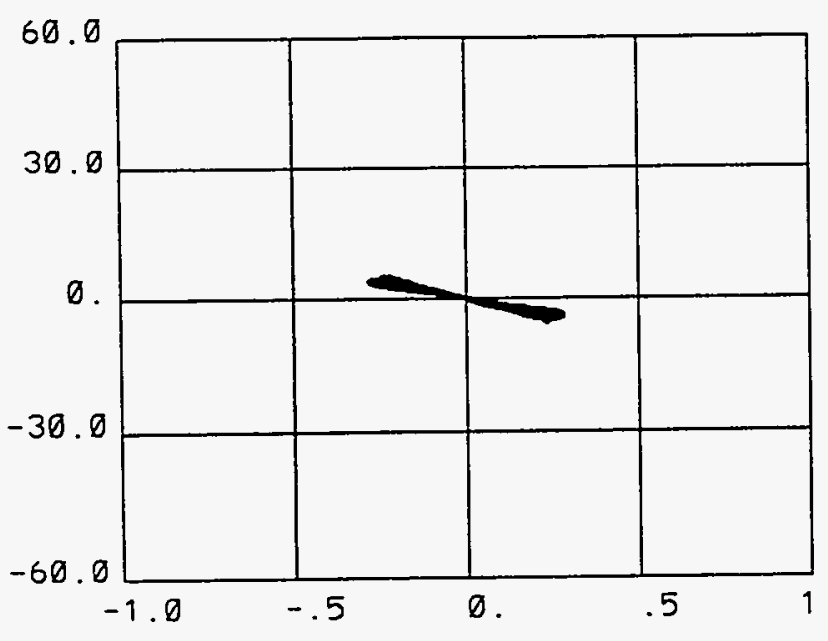

$x$ vs. $x$ element 2

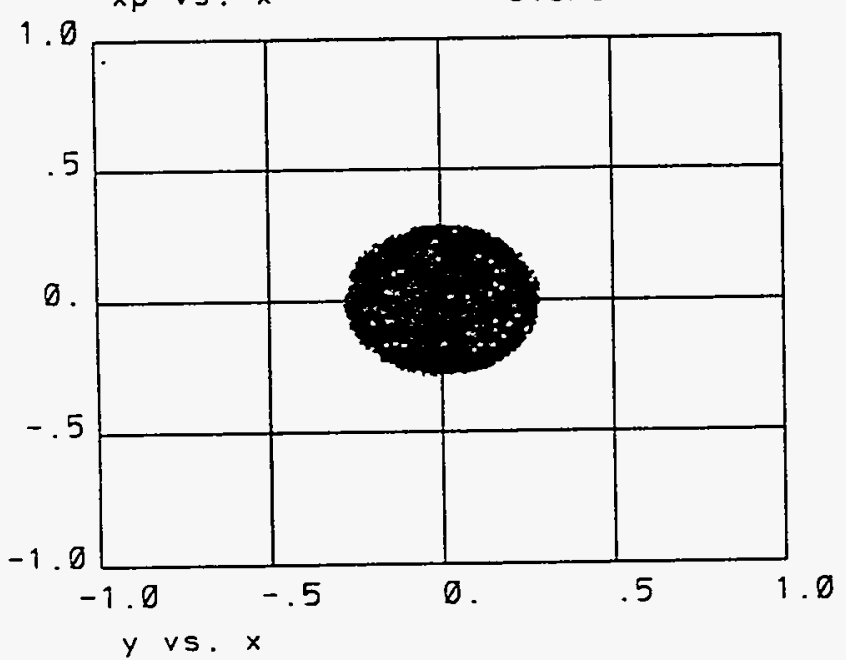

15.15ps G(10),1 nC, phi $=43.1^{\circ}$
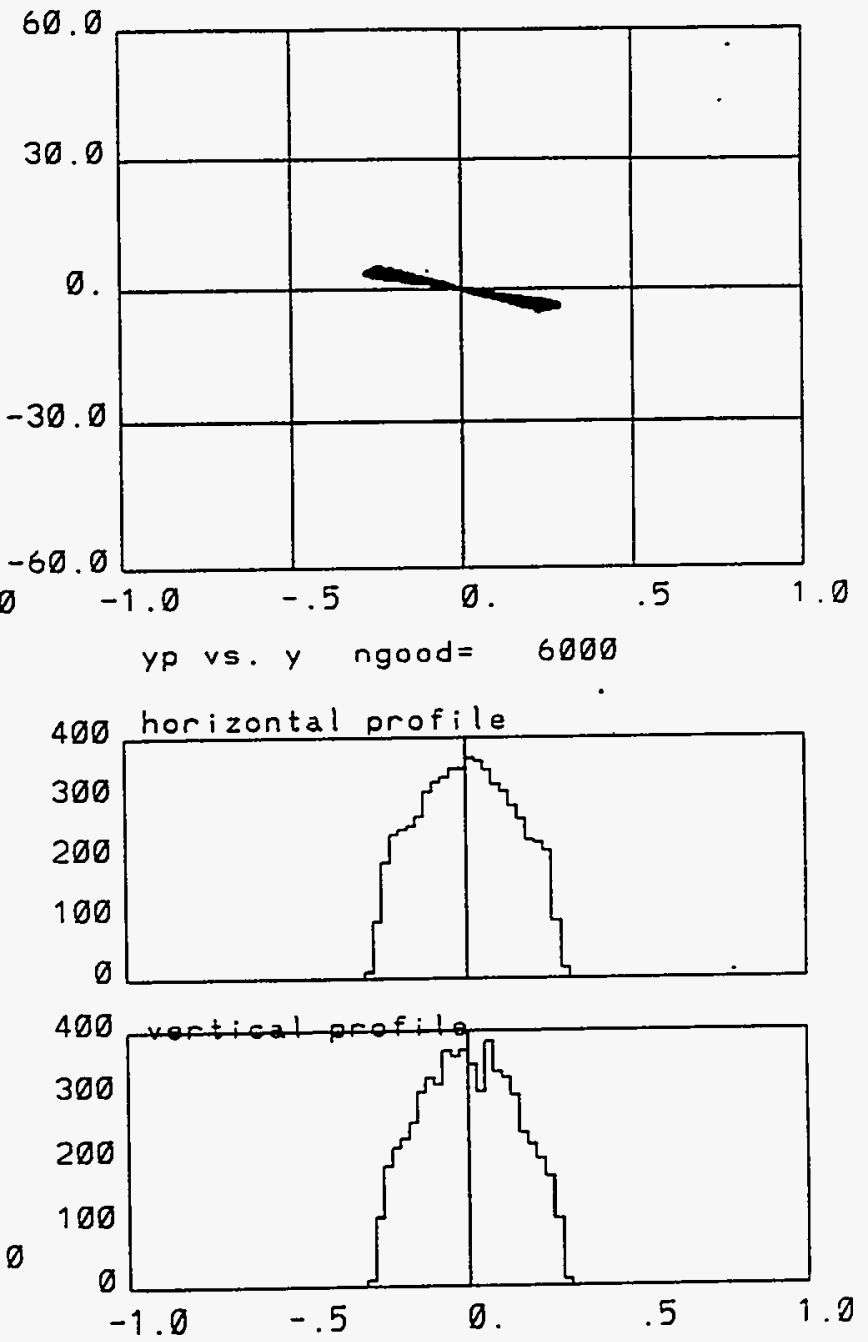
Figure $11-3$ shows the beam profile at position $(n e=3) z=5.2$, with the Inline-Injection system of Solenoid + Gun + Solenoid combination inline with the linac. With Solenoid current of $I=3000$ amp.

- BNL RF GUN
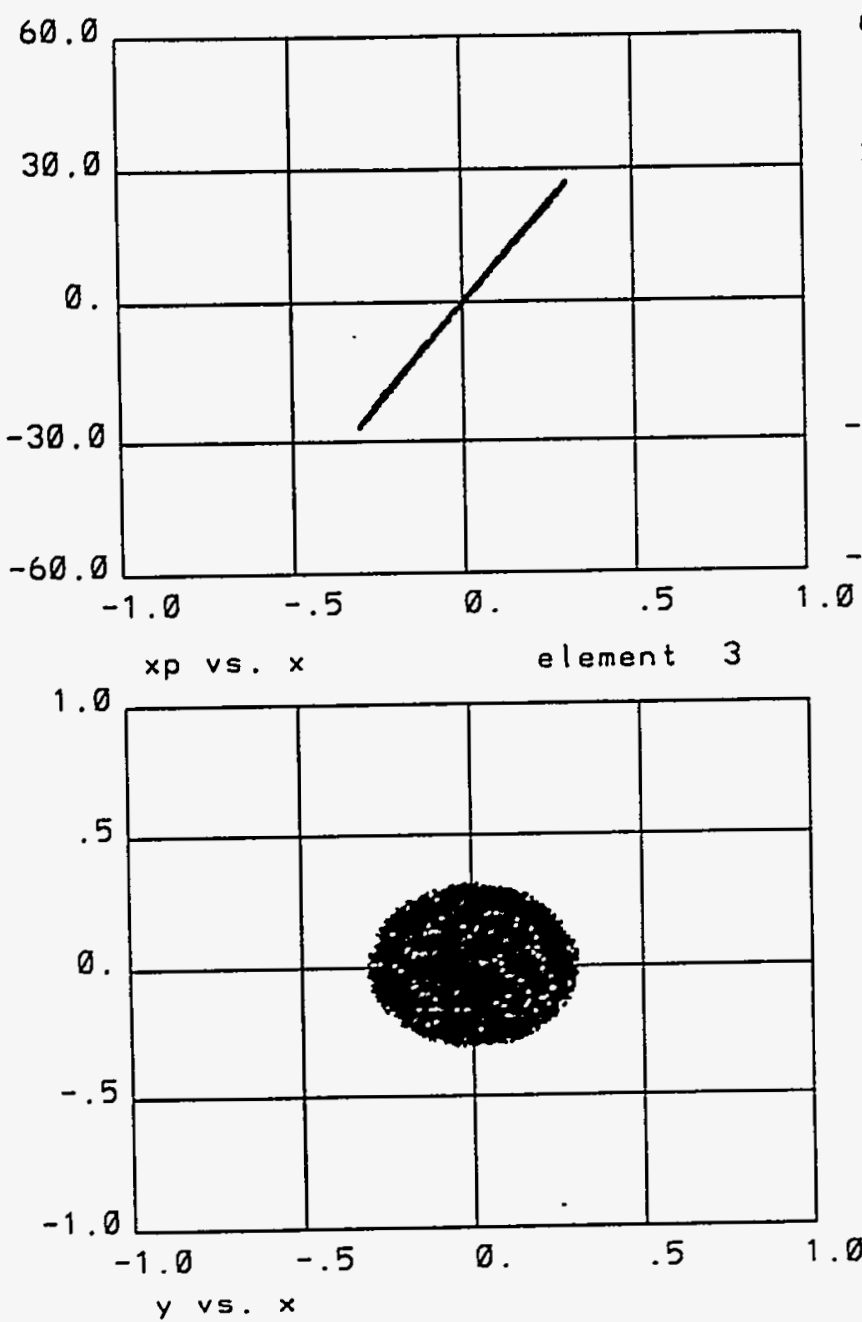

$5.15 p s G(10), 1 n C, p h i=43.1^{\circ}$

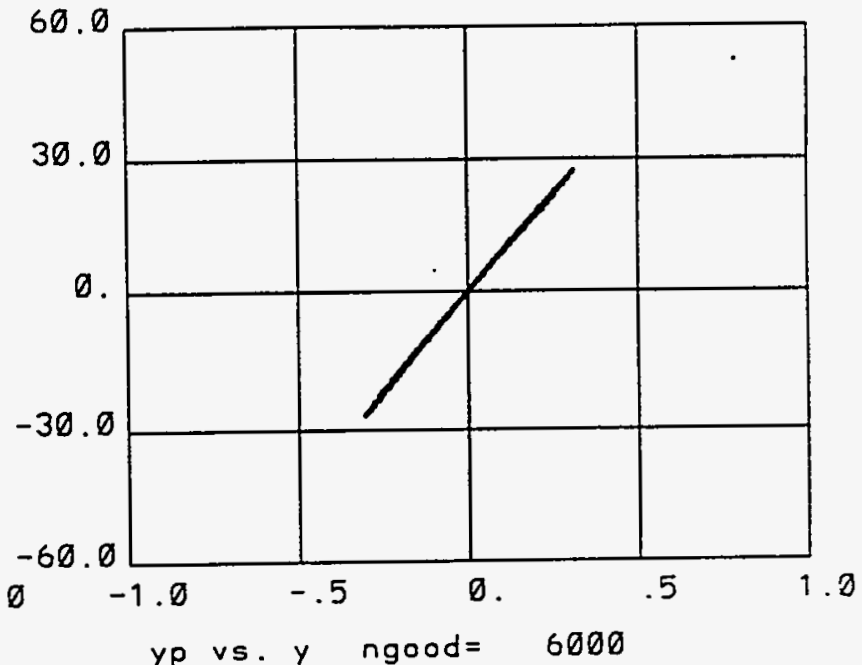

yp vs. y ngood $=6000$

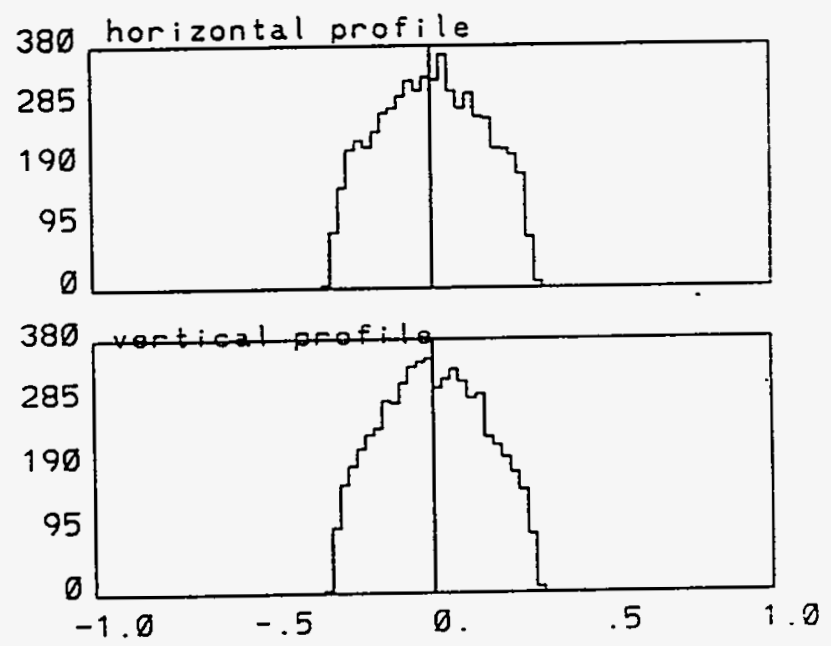


Figure 11-4 shows the beam profile at position $(n e=4) z=7.9$, with the Inline-Injection system of Solenoid + Gun + Solenoid combination inline with the linac. With Solenoid current of $I=3000$ amp.

- BNL RF GUN ,
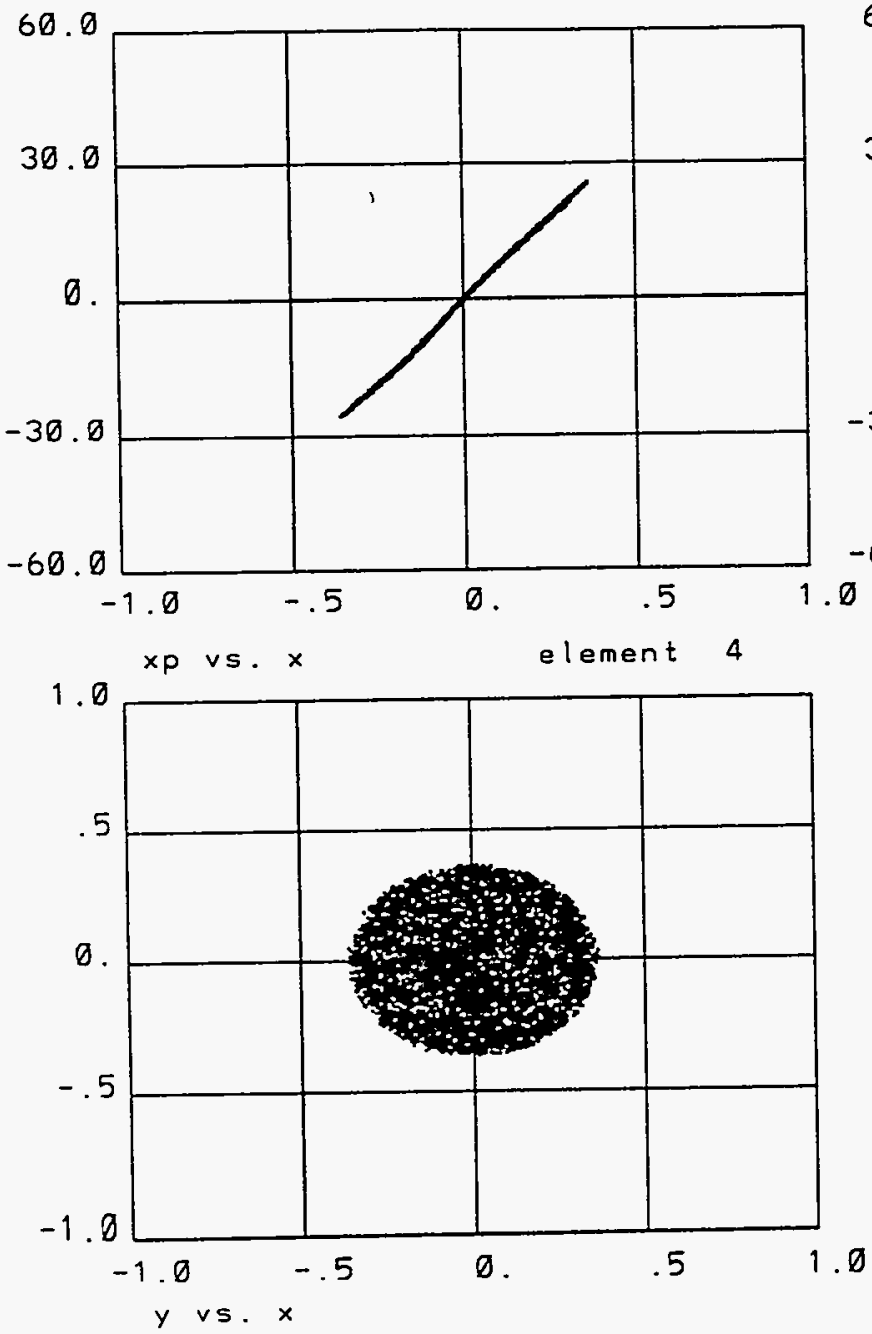

$5.15 p s \mathrm{G}(10), 1 \mathrm{nC}, \mathrm{phi}=43.)^{\text {. }}$
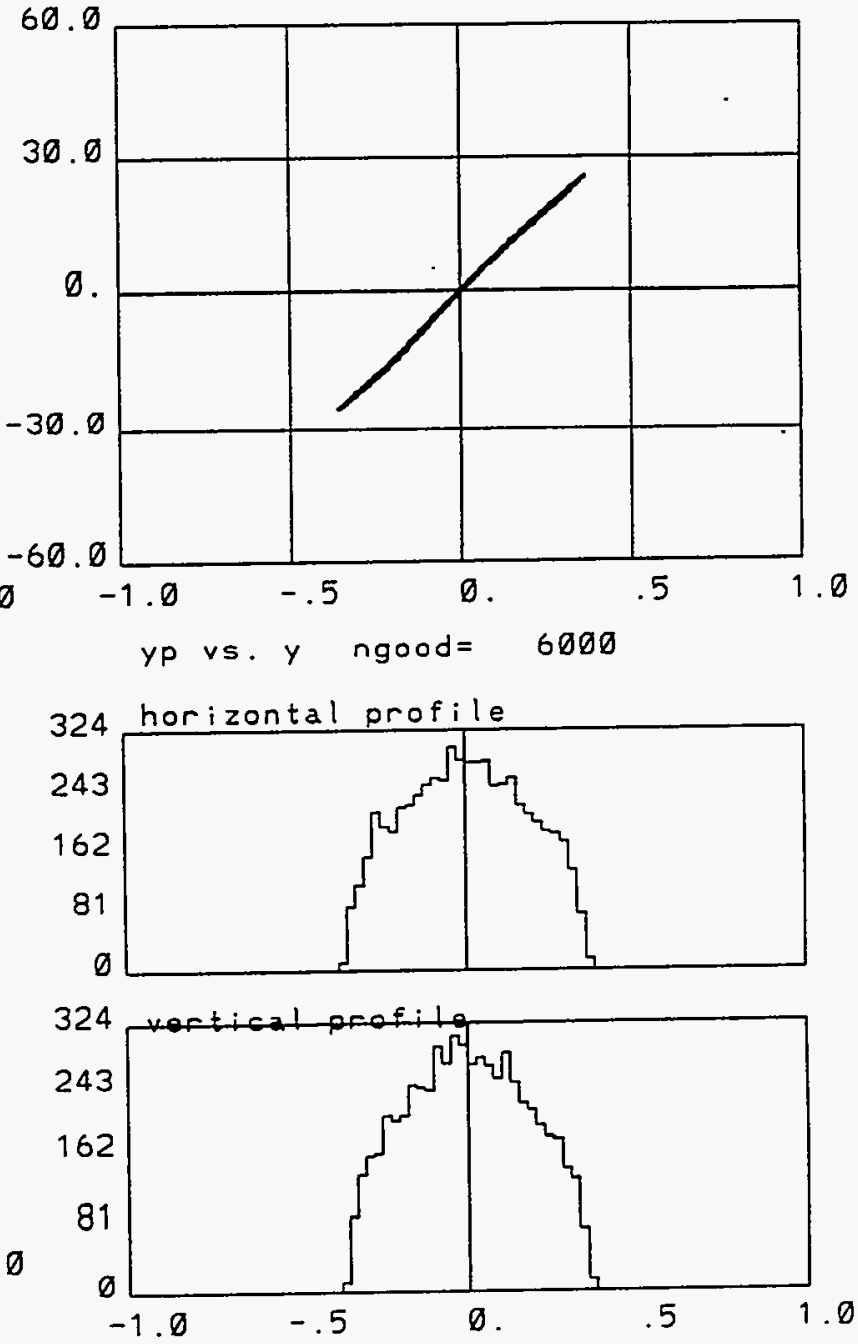
Figure $11-5$ shows the beam profile at position $(n e=5) z=10$, with the Inline-Injection system of Solenoid + Gun + Solenoid combination inline with the linac. With Solenoid current of $I=3000 \mathrm{amp}$.

'BNL RF GUN $(R=.09$ gaus $(1 \mathrm{~mm}), 5.15 \mathrm{ps} G(10), 1 \mathrm{nC}$, phi=43.1.
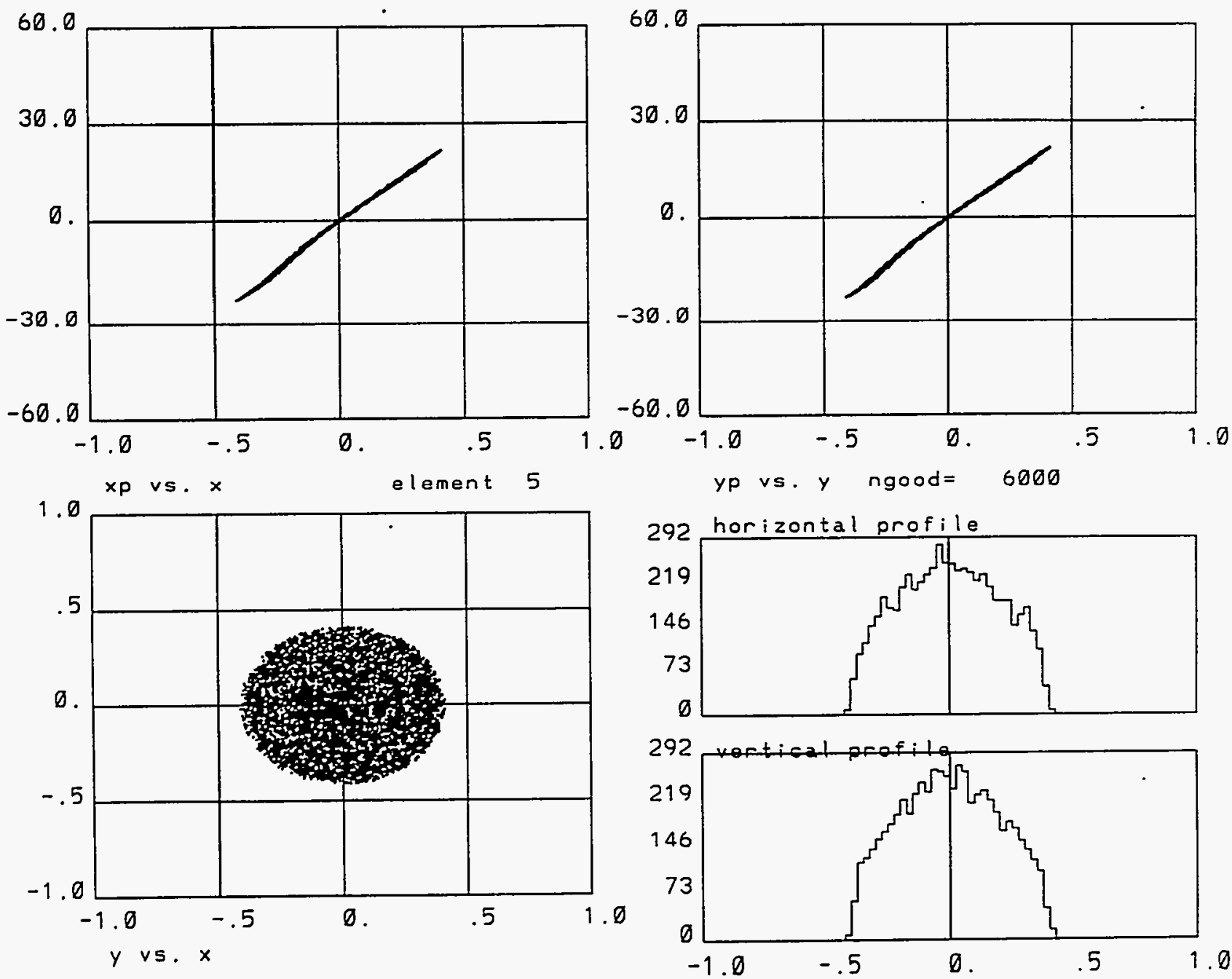
Figure $11-6$ shows the beam profile at position $(n e=10) \quad z=20$, with the Inline-Injection system of Solenoid + Gun + Solenoid combination inline with the linac. With Solenoid current of $I=3000$ amp.

-BNL RF GUN

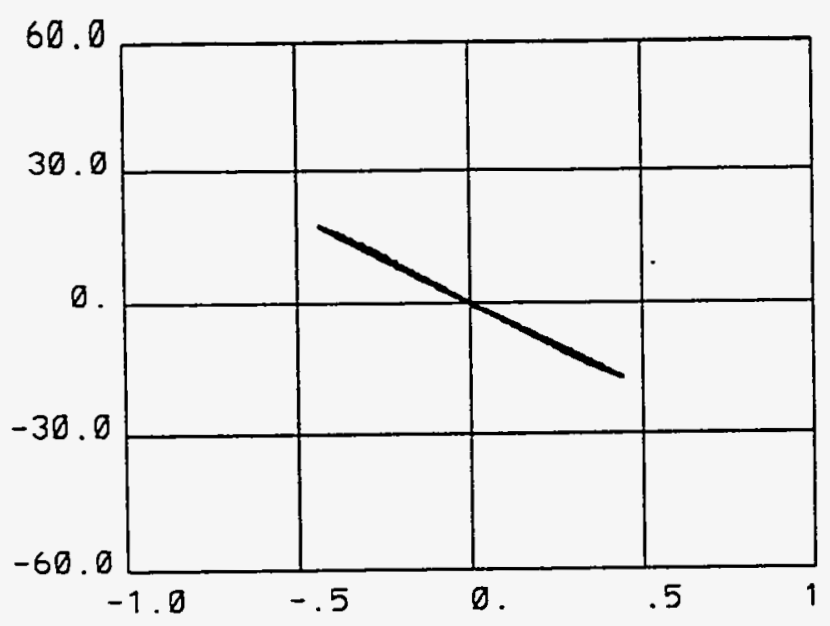

$x p$ vs. $x \quad$ element 10

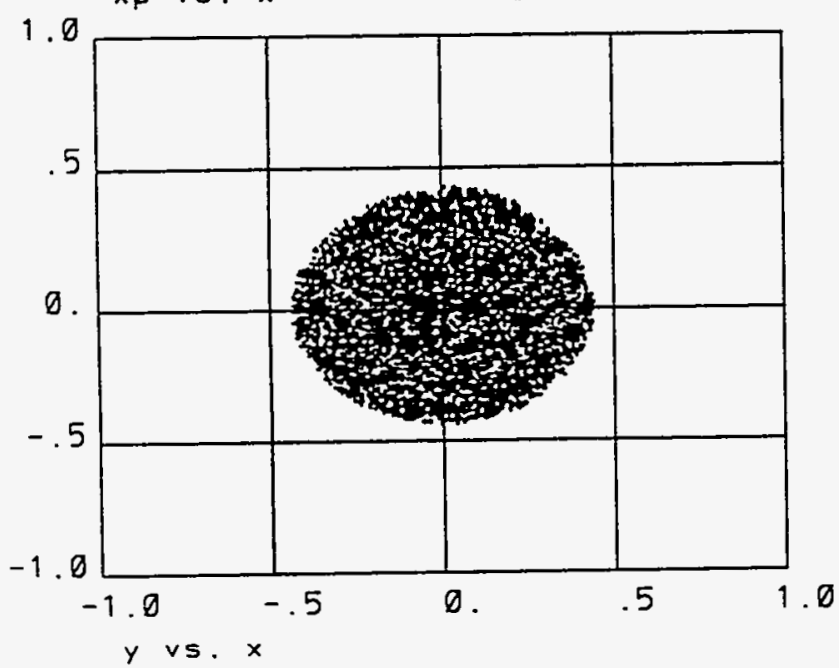

,5.15ps $G(10), 1$ nC, phi $=43.1^{\circ}$
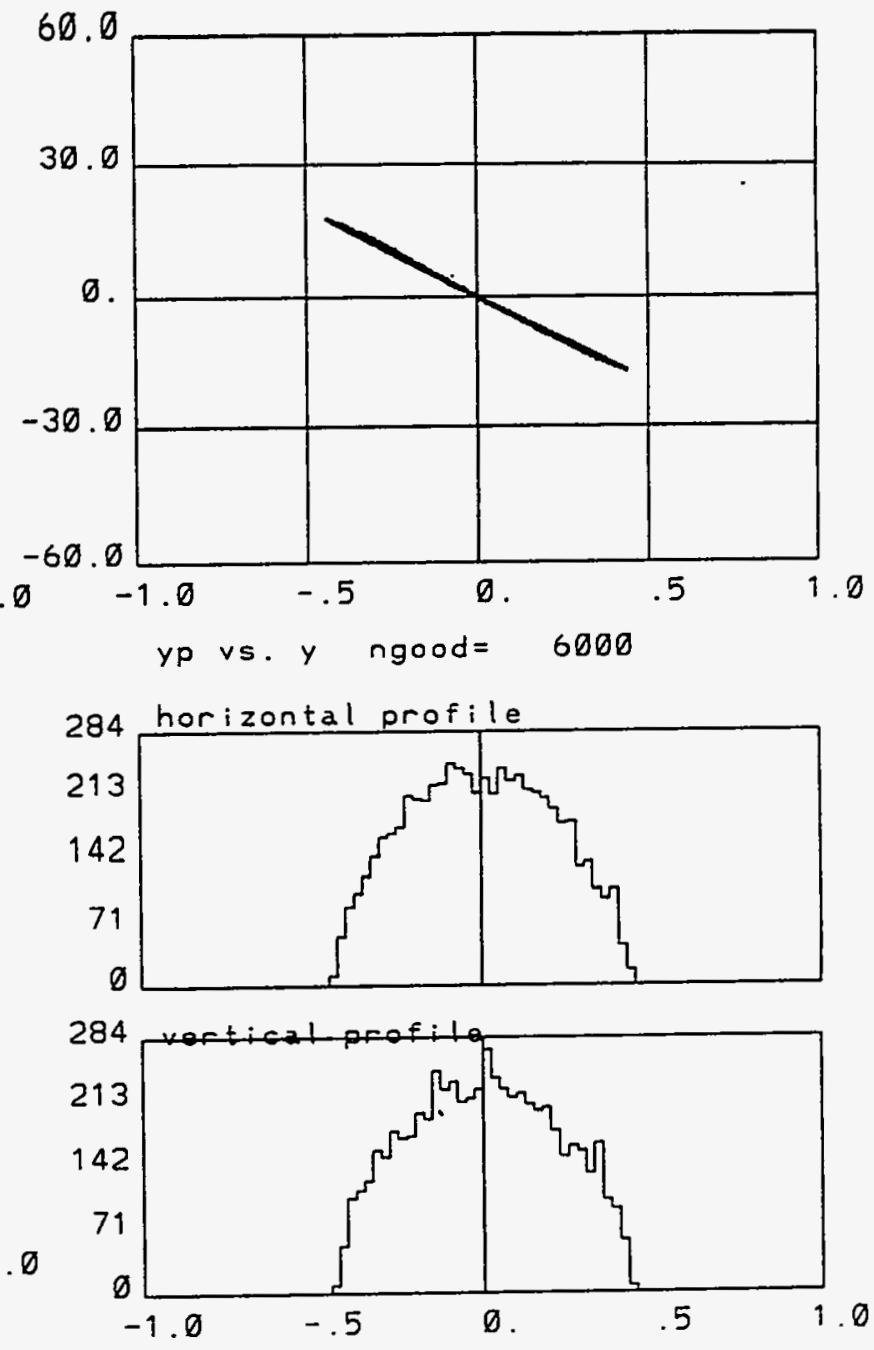
Figure $11-7$ shows the beam profile at position $(n e=20) \quad z=40$, with the Inline-Injection system of Solenoid + Gun + Solenoid combination inline with the linac. With Solenoid current of $I=3000$ amp.

- BNL RF GUN
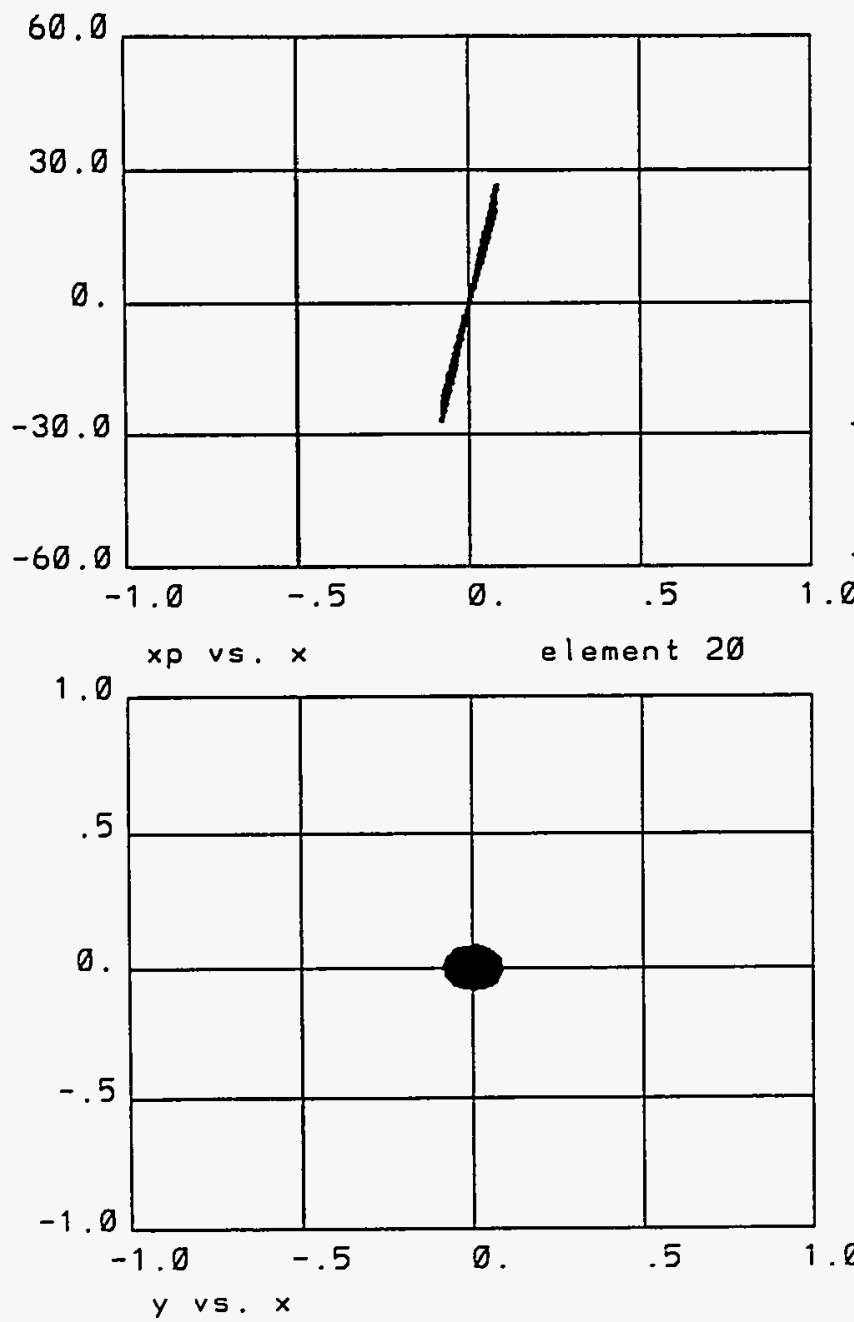

5.15ps $G(10), 1$ nC, phi=43.1.

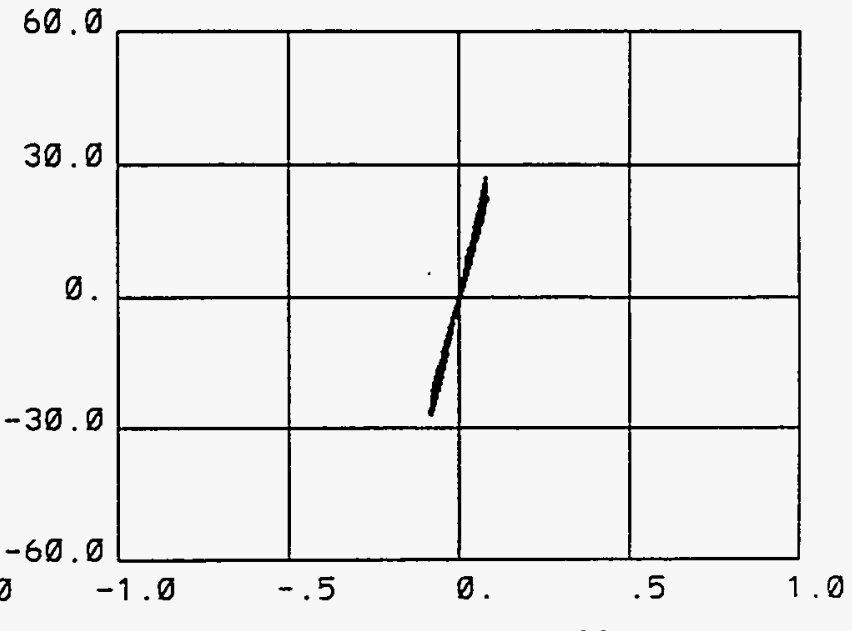

yp vs. y ngood $=6000$
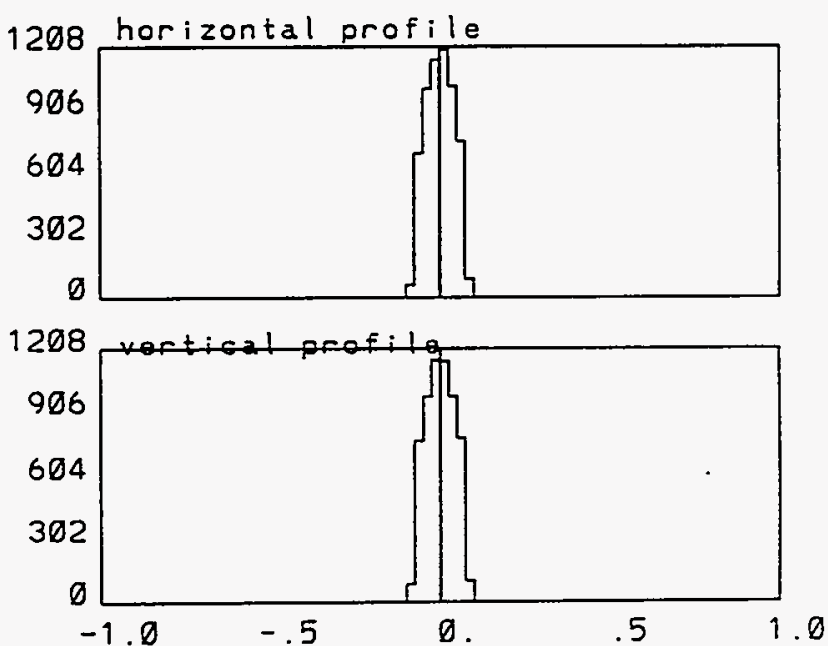
Figure 11-8 shows the beam profile at position $(n e=30) z=70$, with the Inline-Injection system of Solenoid + Gun + Solenoid combination inline with the linac. With Solenoid current of $I=3000 \mathrm{amp}$.

- BNL RF GUN

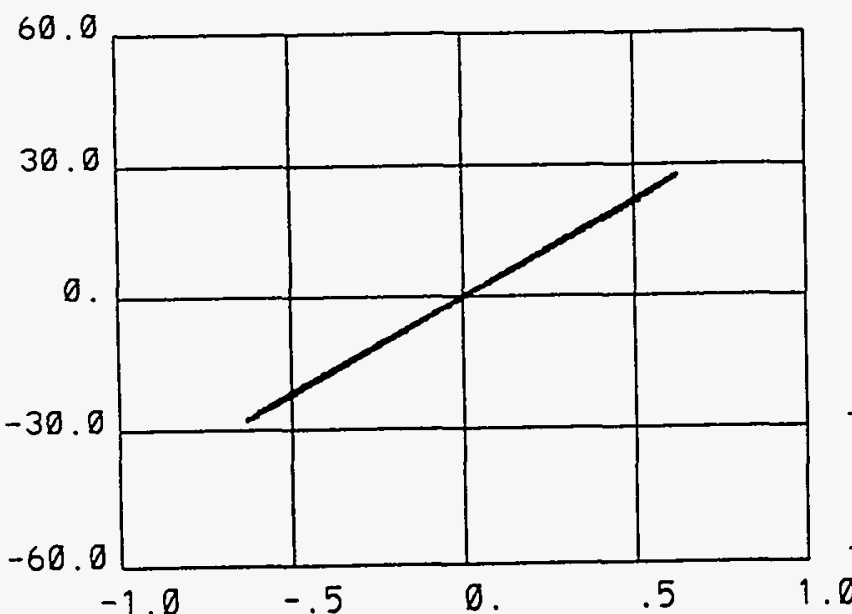

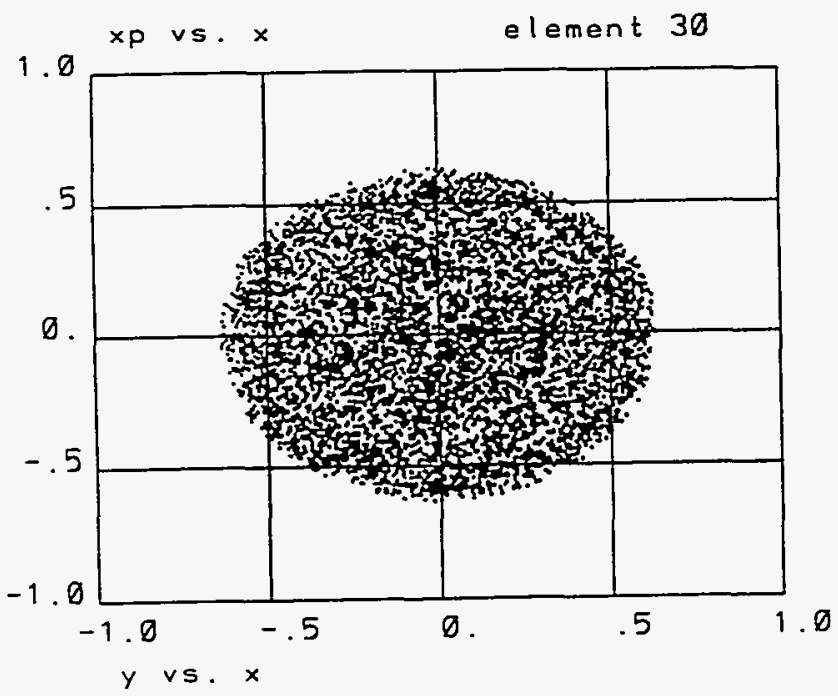

,5.15ps $G(10), 1$ nC, phi $=43.)^{\circ}$

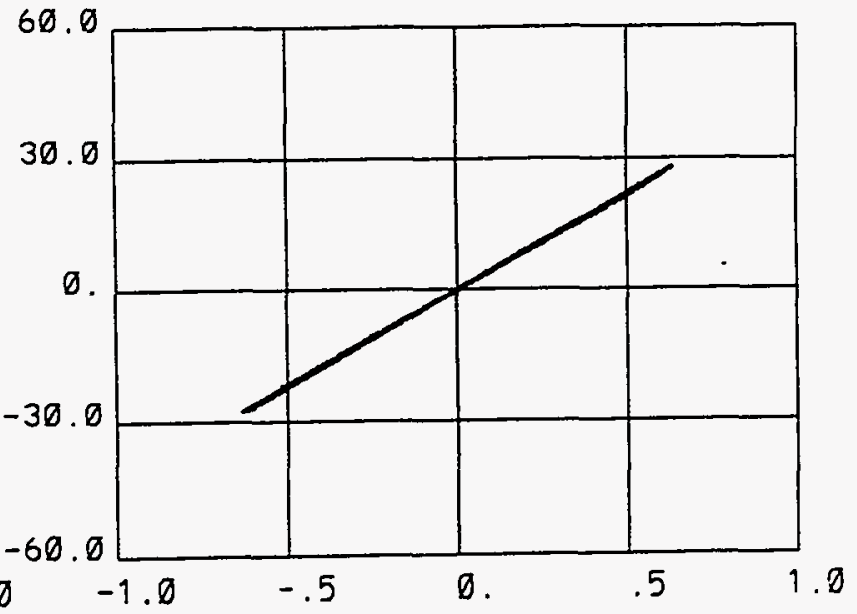

yp vs. y ngood $=6000$
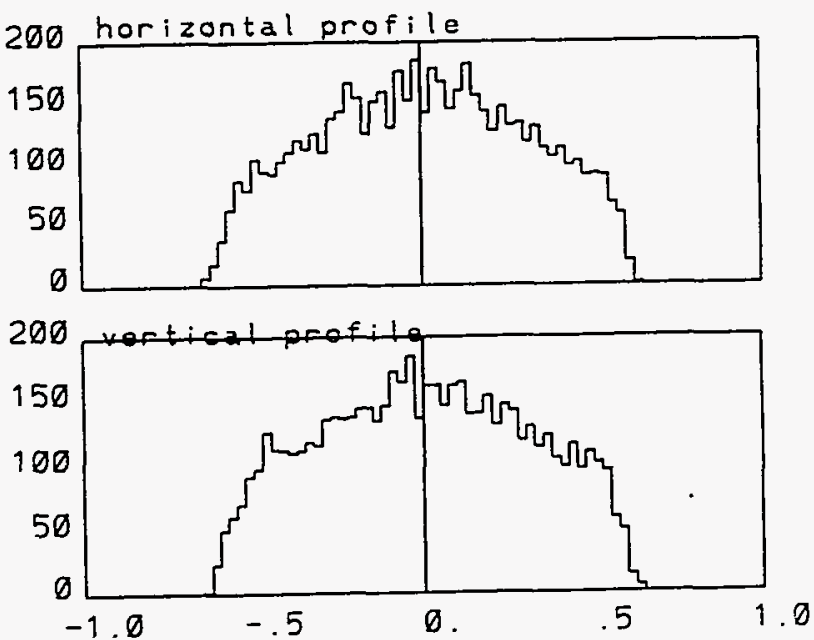
Figure 11-9 shows the beam profile at position $(n e=34) z=90$, with the Inline-Injection system of Solenoid + Gun + Solenoid combination inline with the linac. With Solenoid current of $I=3000 \mathrm{amp}$.

-BNL RF GUN

60.0

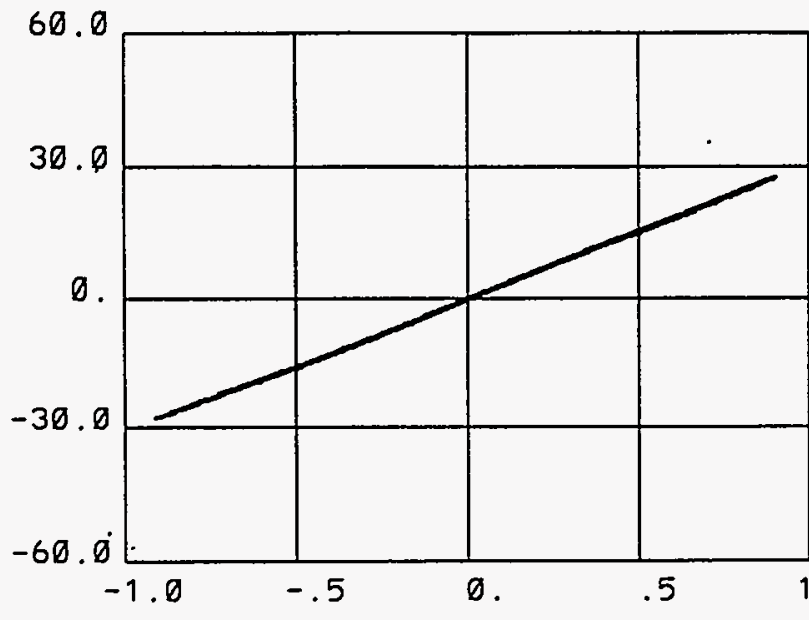

$x$ vs. $x$ element 34

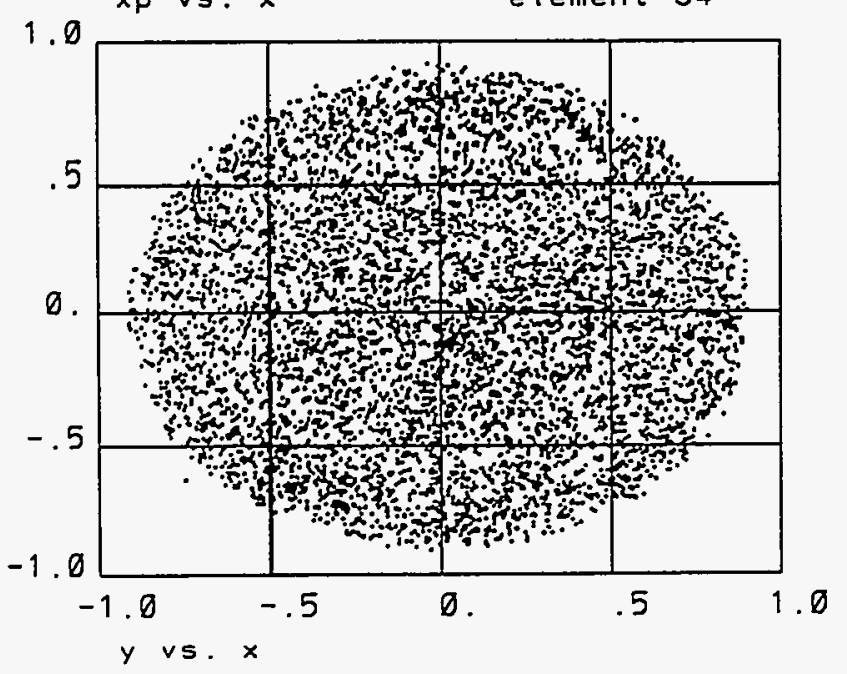

,5.15ps $G(10), 1 \mathrm{nC}$, phi $=43.)^{\prime}$

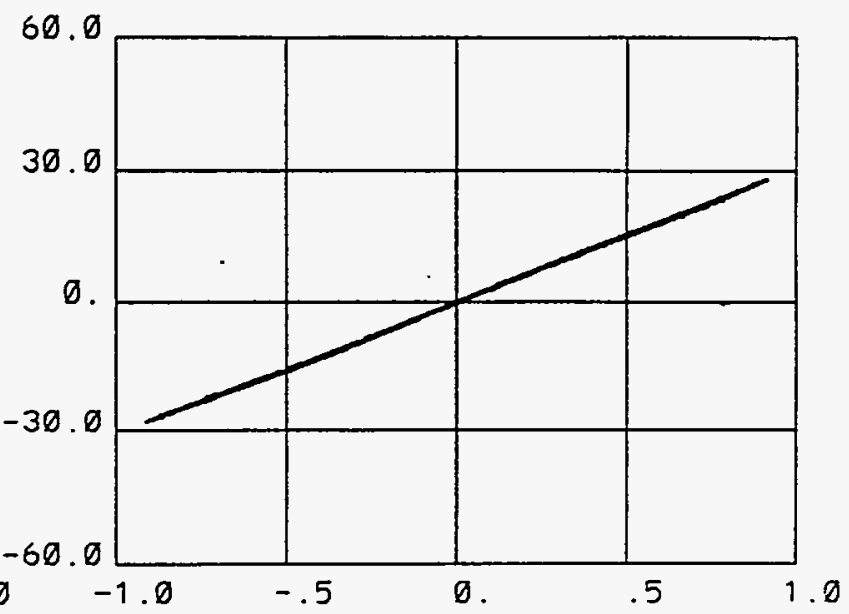

yp vs. y ngood $=6000$
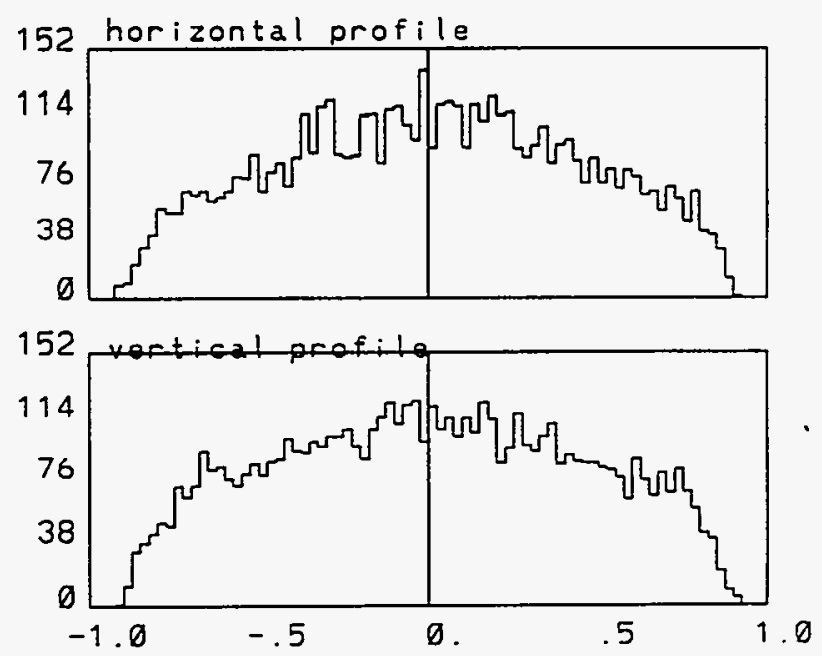
Figures 12-1 to 12-10 shows the beam profile at various positions (distance $\mathrm{z}$ from the cathode), with the Inline-Injection system of Solenoid + Gun + Solenoid combination inline with the linac. With Solenoid current of $I=3400 \mathrm{amp}$.

- BNL RF GUN
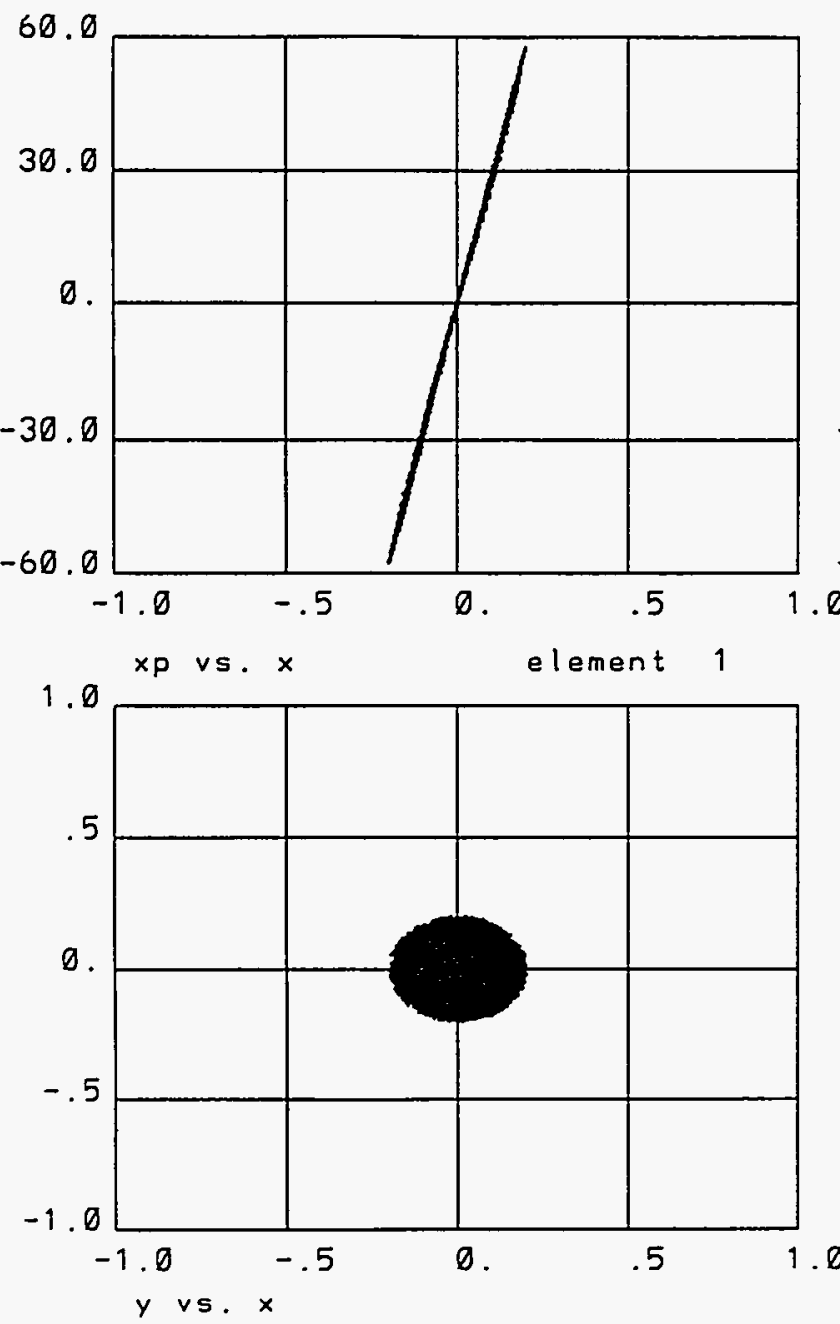

$(0.9 \mathrm{~mm}), 5.15 \mathrm{ps}, 100 \mathrm{MV} / \mathrm{m}, 1 \mathrm{nC}, \mathrm{phi}=43.01$.

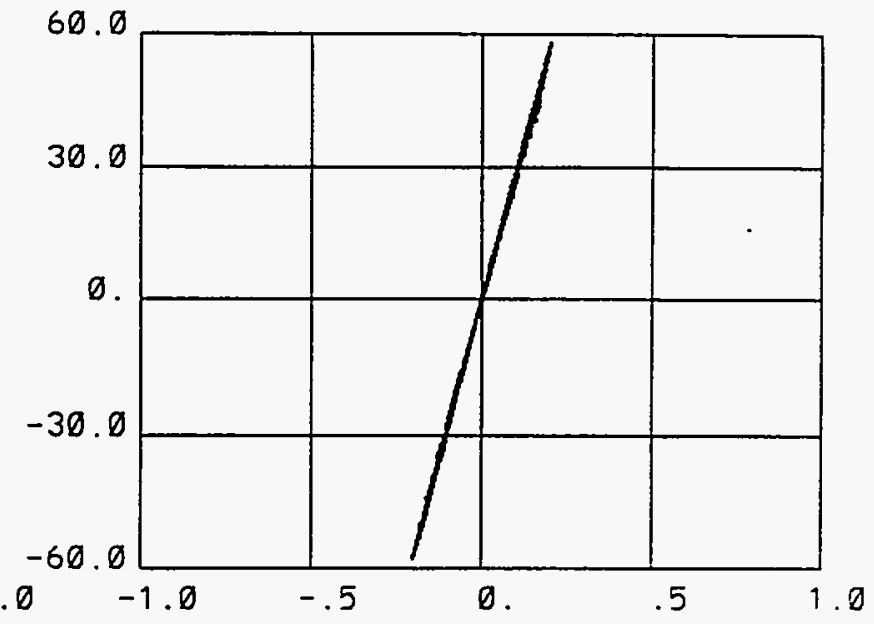

yp vs. y ngood $=6000$
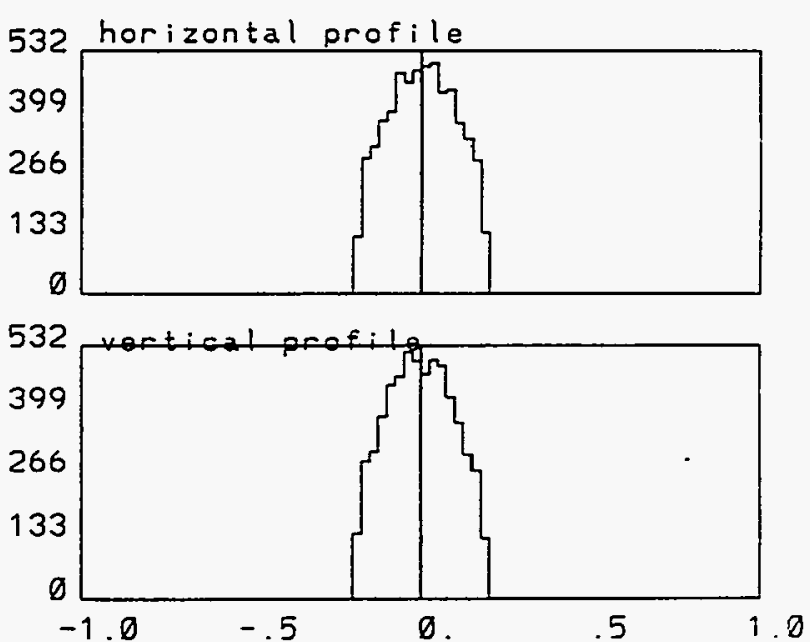

Figure $12-1$ shows the beam profile at position $(n e=1) \quad z=0$, with the Inline-Injection system of Solenoid + Gun + Solenoid combination inline with the linac. With Solenoid current of $I=3400$ amp. 
Figure $12-2$ shows the beam profile at position (ne=2) $z=2.6$, with the Inline-Injection system of Solenoid + Gun + Solenoid combination inline with the linac. With Solenoid current of $I=3400 \mathrm{amp}$.

- BNL RF GUN

60.
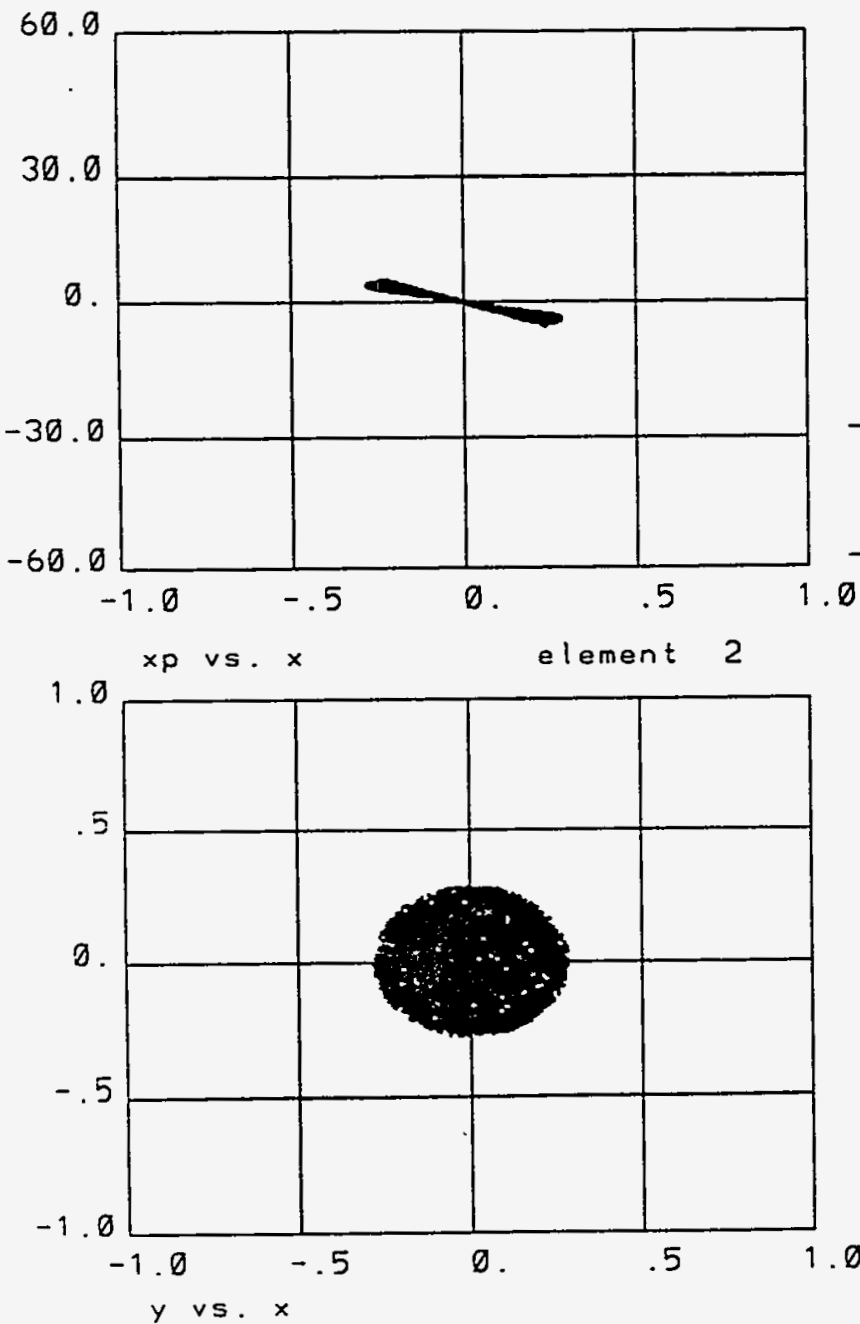

$(0.9 \mathrm{~mm}), 5.15 \mathrm{ps}, 100 \mathrm{MV} / \mathrm{m}, 1 \mathrm{nC}, \mathrm{phi}=43.0) \cdot$
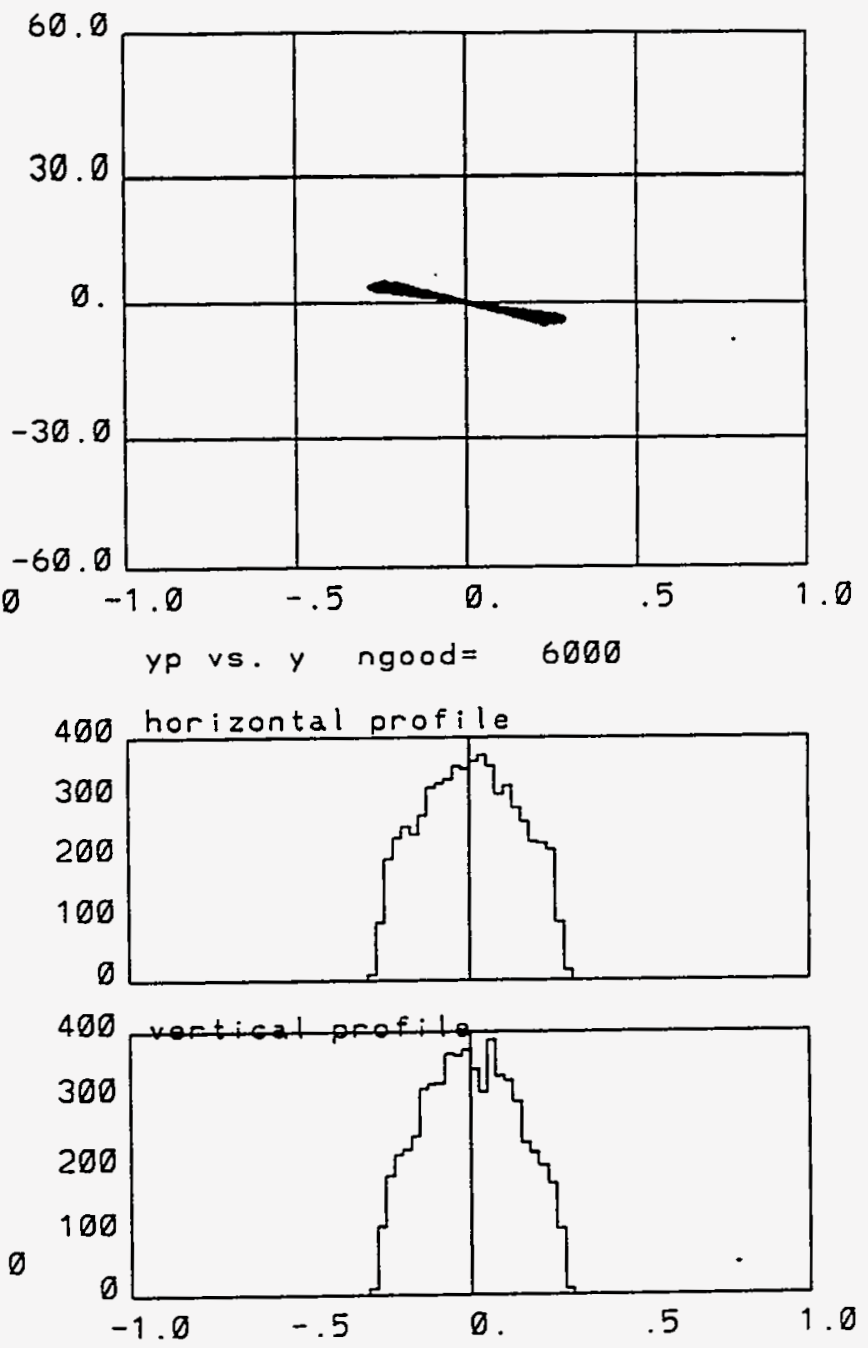
Figure $12-3$ shows the beam profile at position (ne=3) $z=5.2$, with the Inline-Injection system of Solenoid + Gun + Solenoid combination inline with the linac. With Solenoid current of $I=3400$ amp.
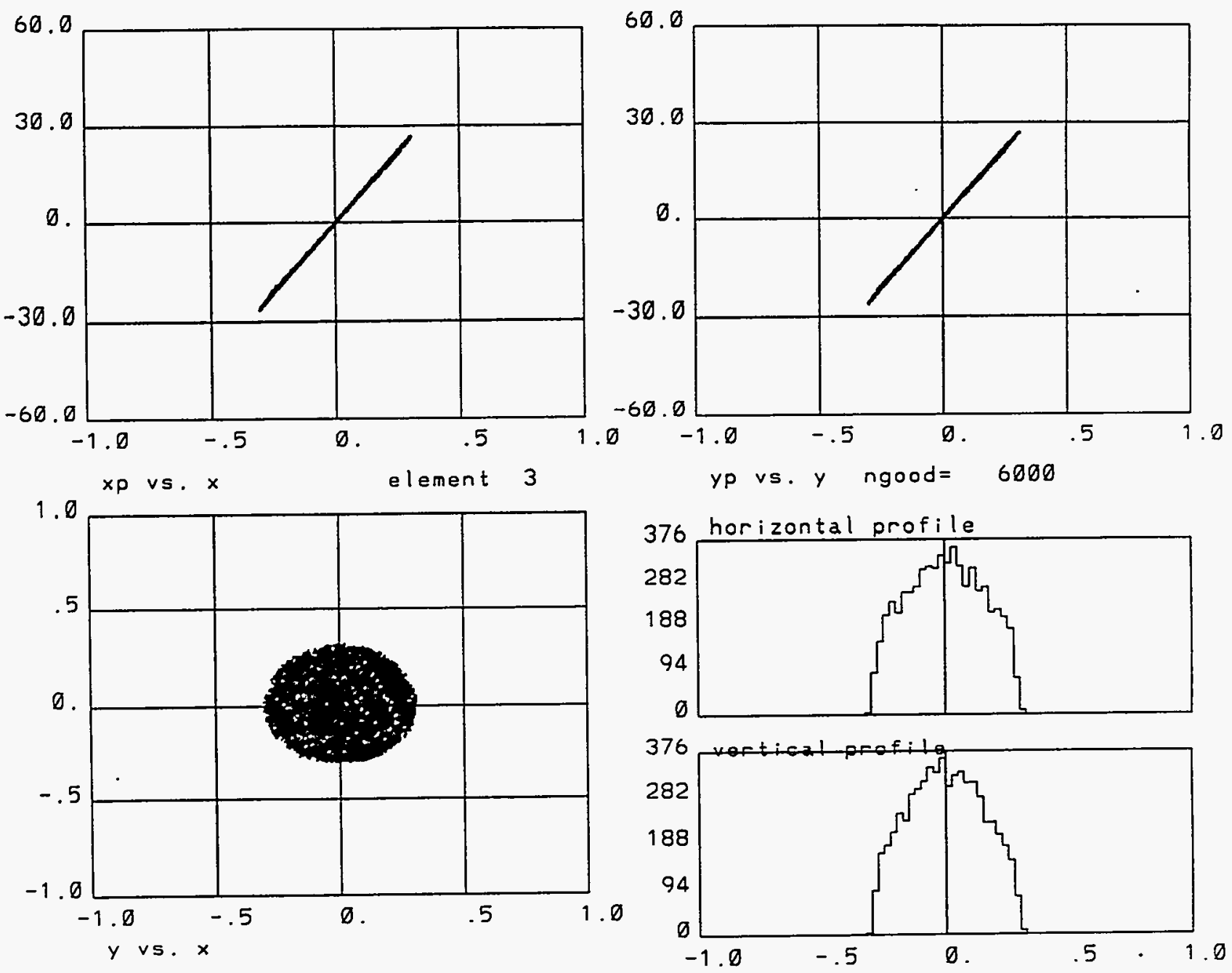
Figure 12-4 shows the beam profile at position $(n e=4) z=7.9$, with the Inline-Injection system of Solenoid + Gun + Solenoid combination inline with the linac. With Solenoid current of $I=3400 \mathrm{amp}$.

- BNL RF GUN 1

$$
60.8
$$
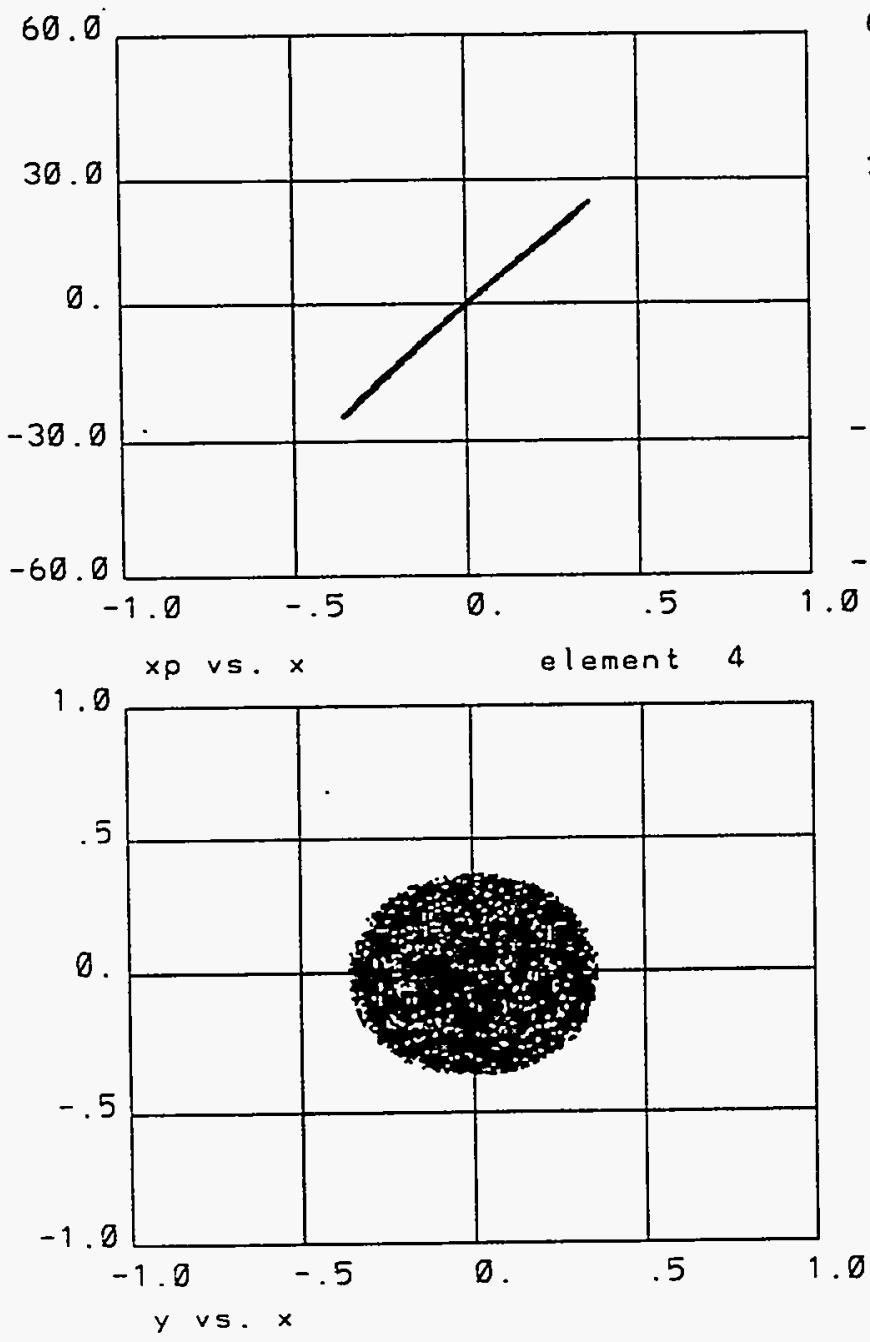

$(0.9 \mathrm{~mm}), 5.15 p s, 100 \mathrm{MV} / \mathrm{m}, 1 \mathrm{nC}, \mathrm{phi}=43.0)$
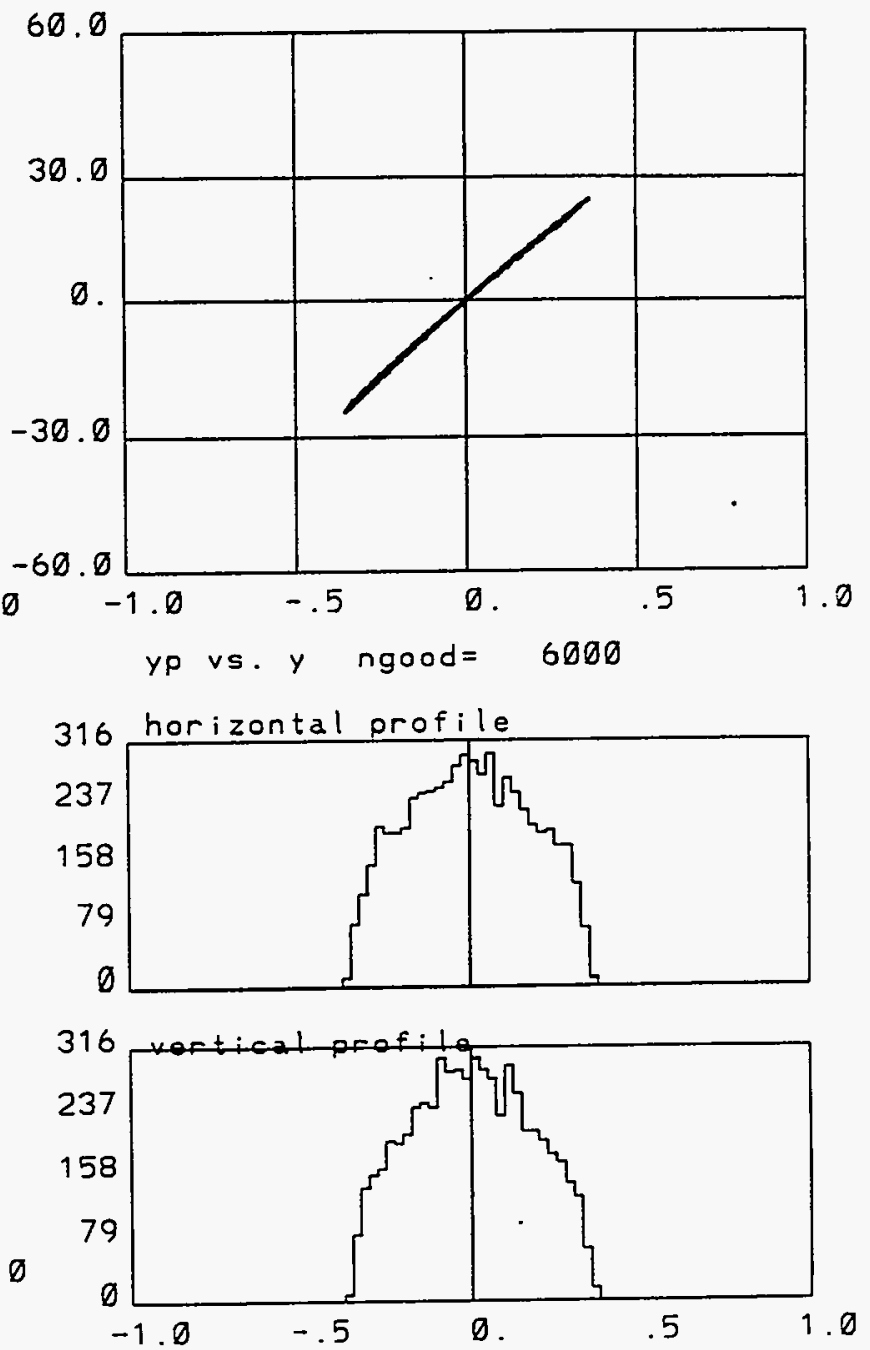
Figure $12-5$ shows the beam profile at position (ne=5) $z=10$, with the Inline-Injection system of Solenoid + Gun + Solenoid combination inline with the linac. With Solenoid current of $I=3400 \mathrm{amp}$.

- BNL RF GUN
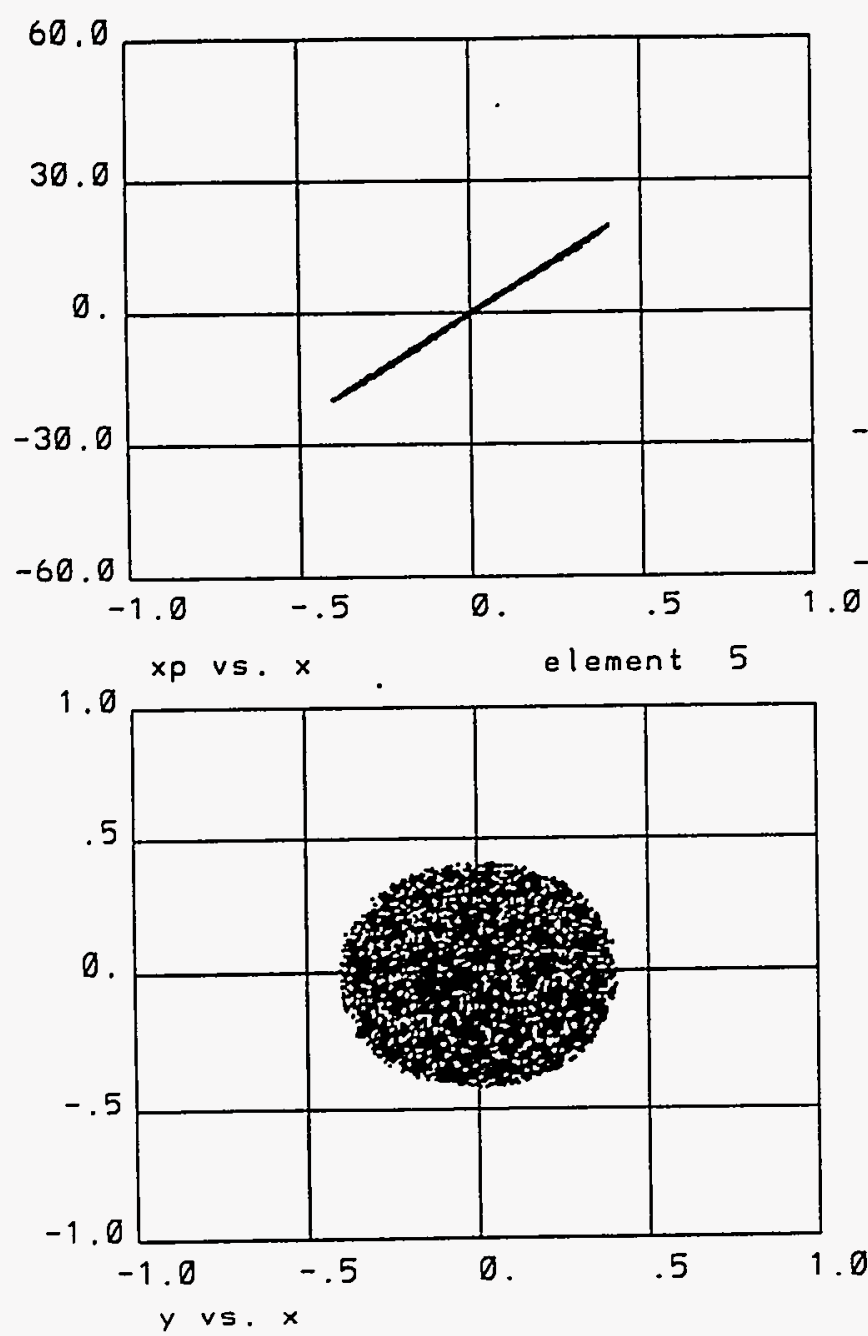

$(0.9 \mathrm{~mm}), 5.15 p s, 100 M V / m$, inC, phi $=43.0)$

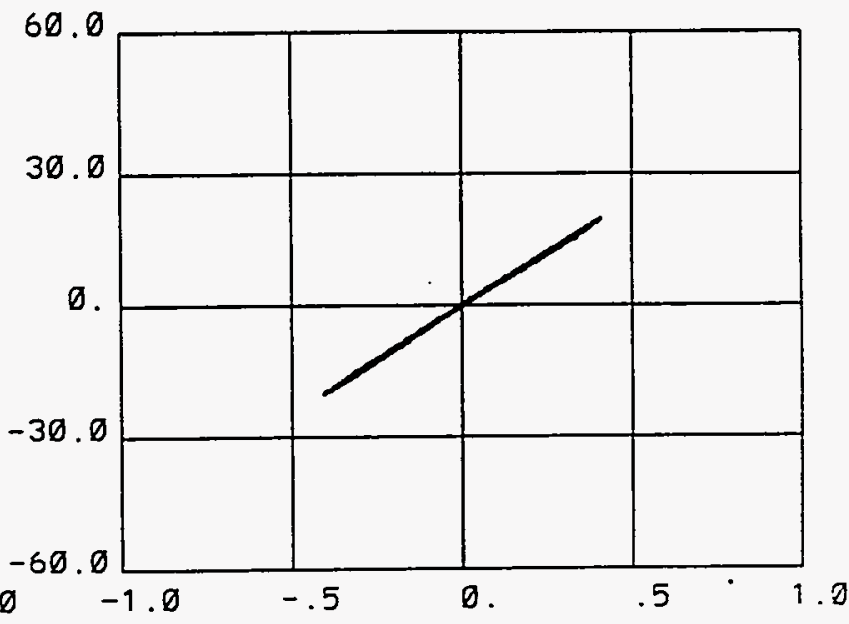

yp vs. y ngood $=6000$
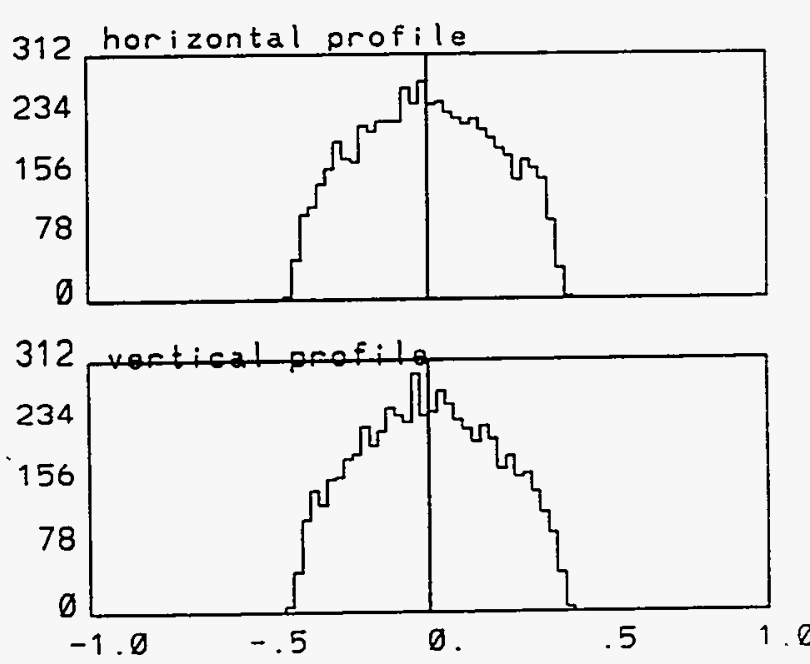
Figure $12-6$ shows the beam profile at position $(n e=10) z=20$, with the Inline-Injection system of Solenoid + Gun + Solenoid combination inline with the linac. With Solenoid current of $I=3400$ amp.

-BNL RF GUN

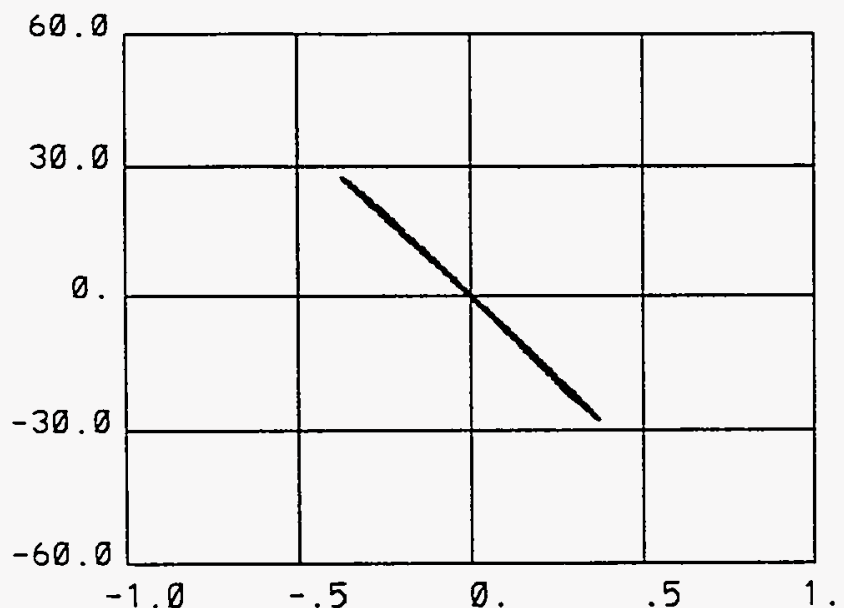

$x p$ vs. $x$

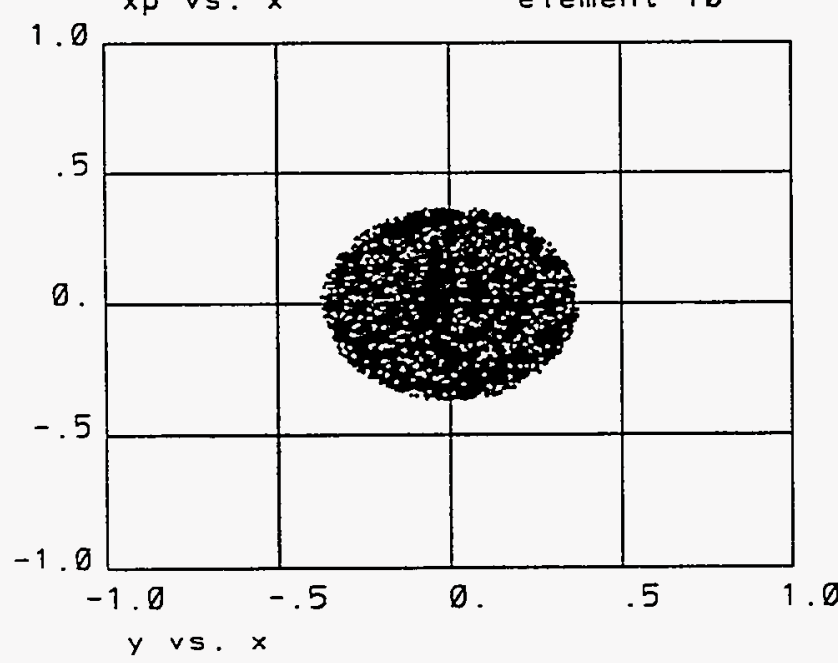

$(0.9 \mathrm{~mm}), 5.15 \mathrm{ps} .100 \mathrm{MV} / \mathrm{m}, 1 \mathrm{nC}, \mathrm{phi}=43.0)$

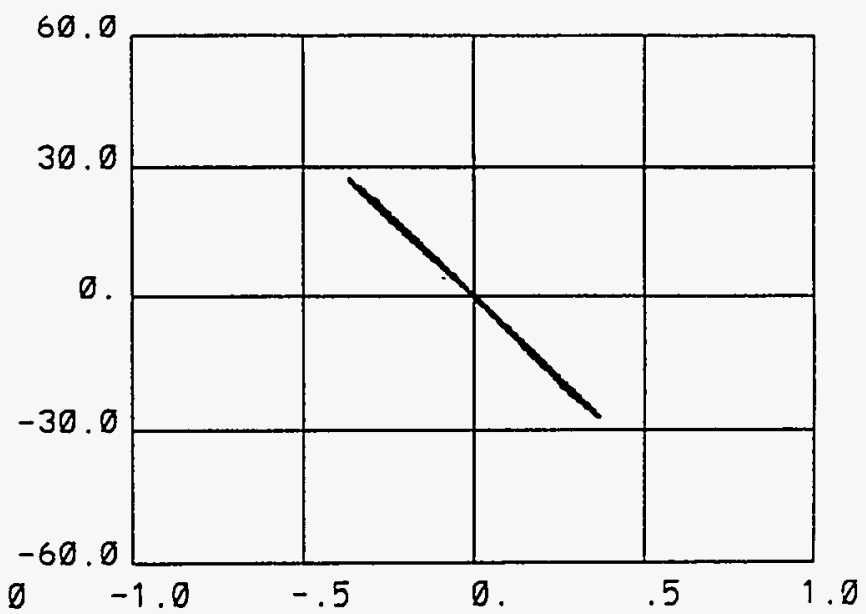

yp vs. y ngood $=6000$
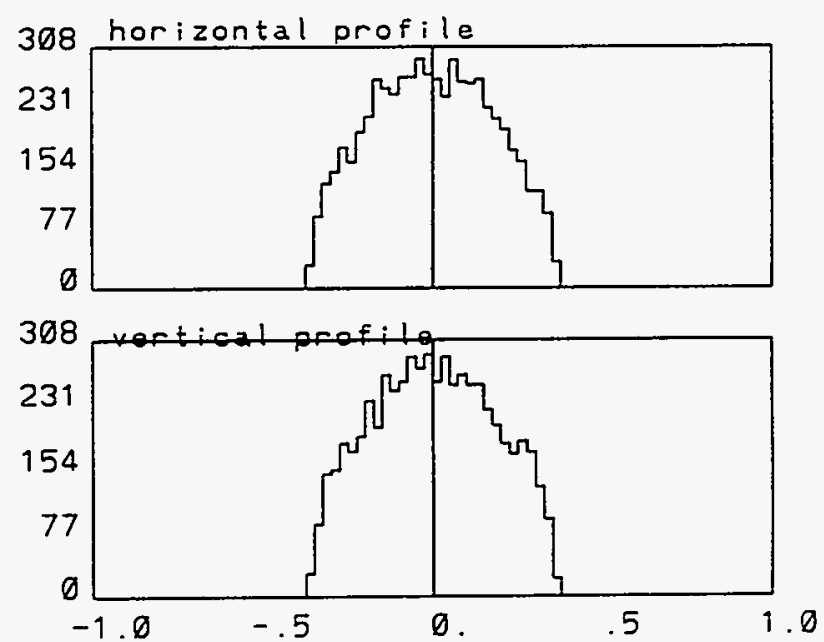
Figure $12-7$ shows the beam profile at position $(n e=20) z=40$, with the Inline-Injection system of Solenoid + Gun + Solenoid combination inline with the linac. With Solenoid current of $I=3400 \mathrm{amp}$.

'BNL RF GUN

$$
60.0
$$

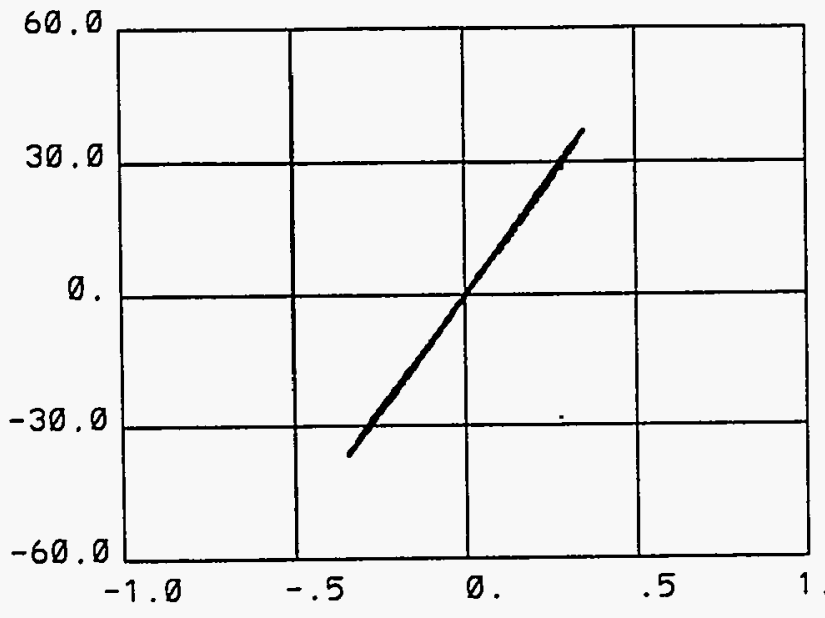

$x p \vee s . x$

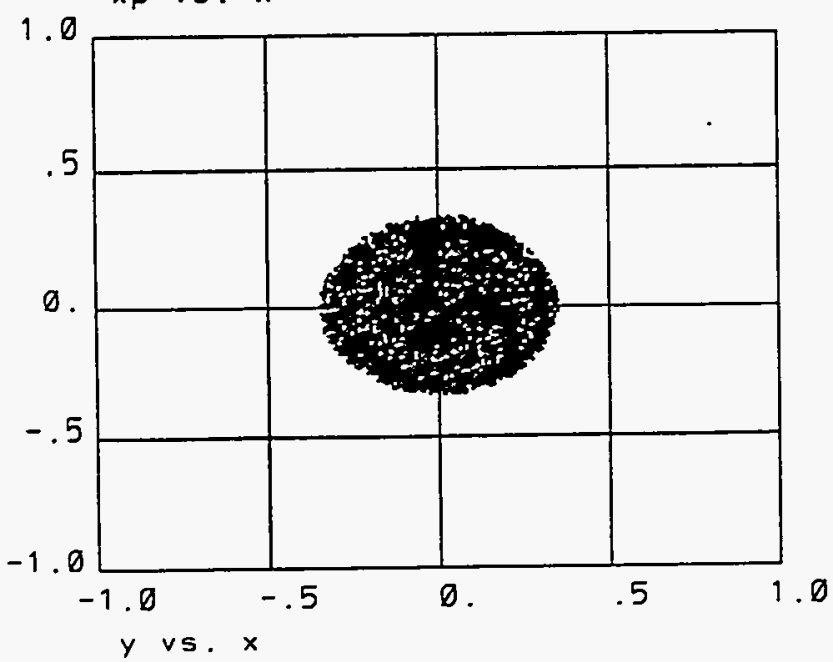

$(0.9 \mathrm{~mm}), 5.15 p s, 100 \mathrm{MV} / \mathrm{m}, 1 \mathrm{nC}, \mathrm{phi}=43.0)^{\circ}$
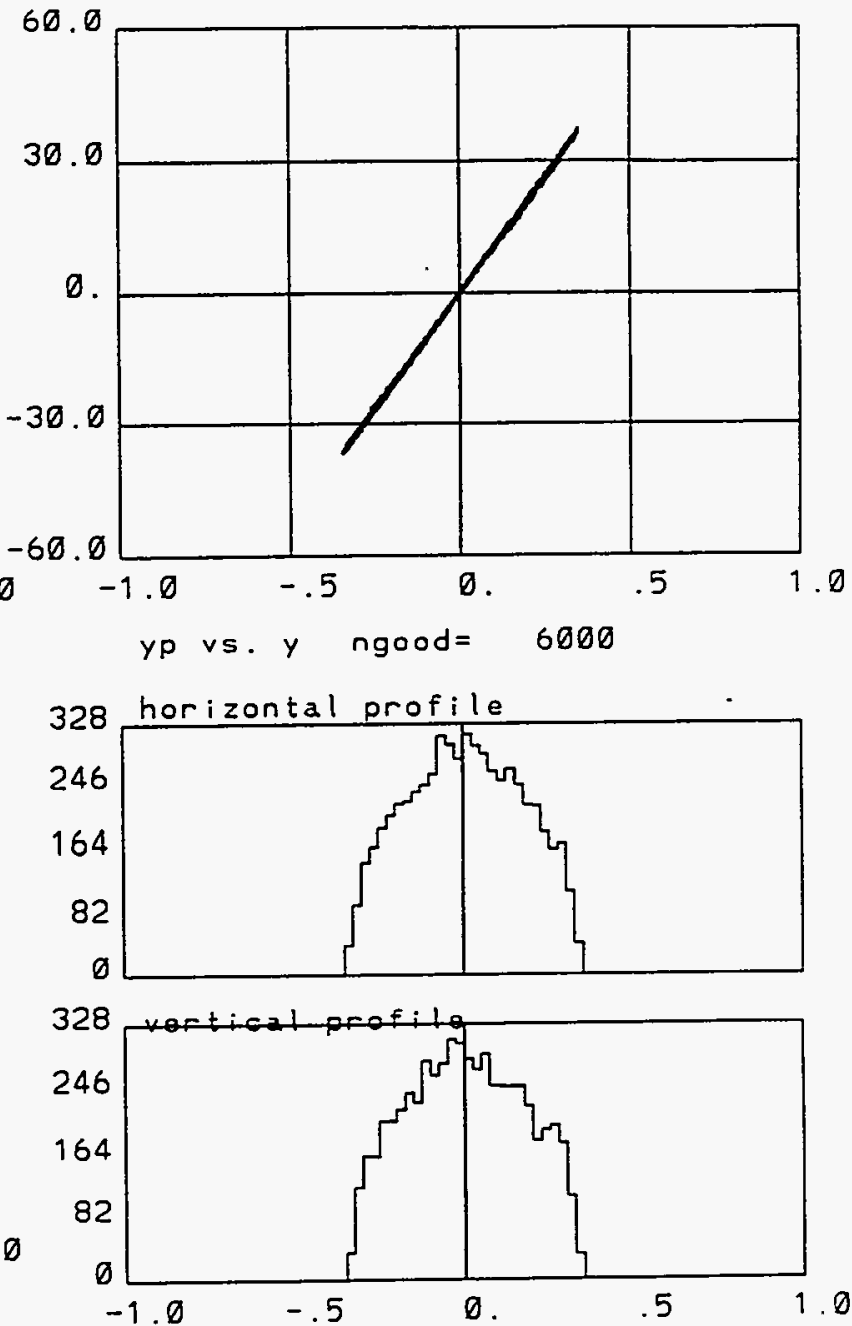
Figure 12-8 shows the beam profile at position $(n e=27) z=58$, with the Inline-Injection system of Solenoid + Gun + Solenoid combination inline with the linac. With Solenoid current of $I=3400 \mathrm{amp}$.

- BNL RF GUN
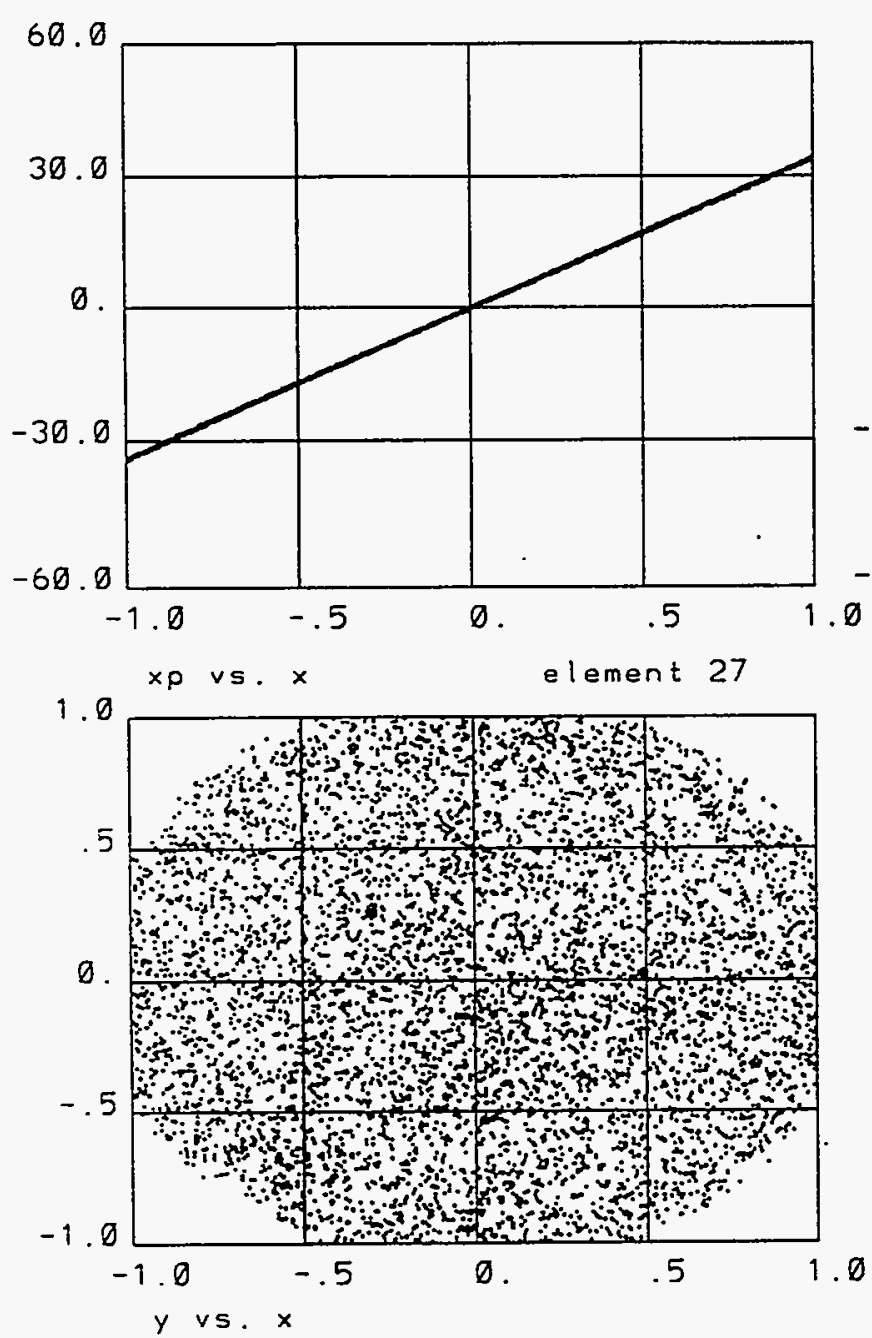

$(0.9 \mathrm{~mm}), 5.15 \mathrm{ps} .100 \mathrm{MV} / \mathrm{m}, 1 \mathrm{nC}$, phi $=43.0)$

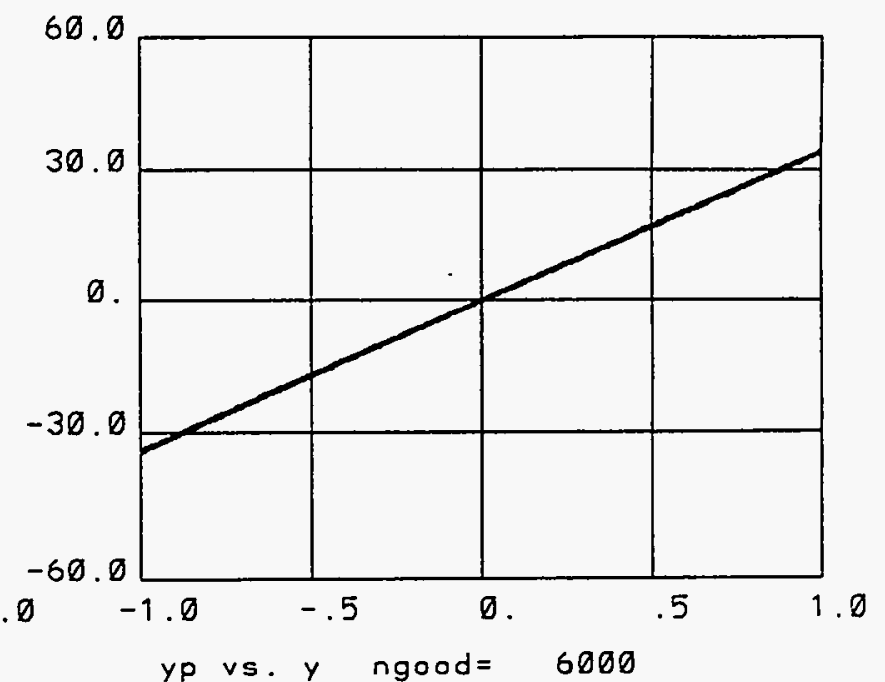

yp vs. y ngood $=6000$
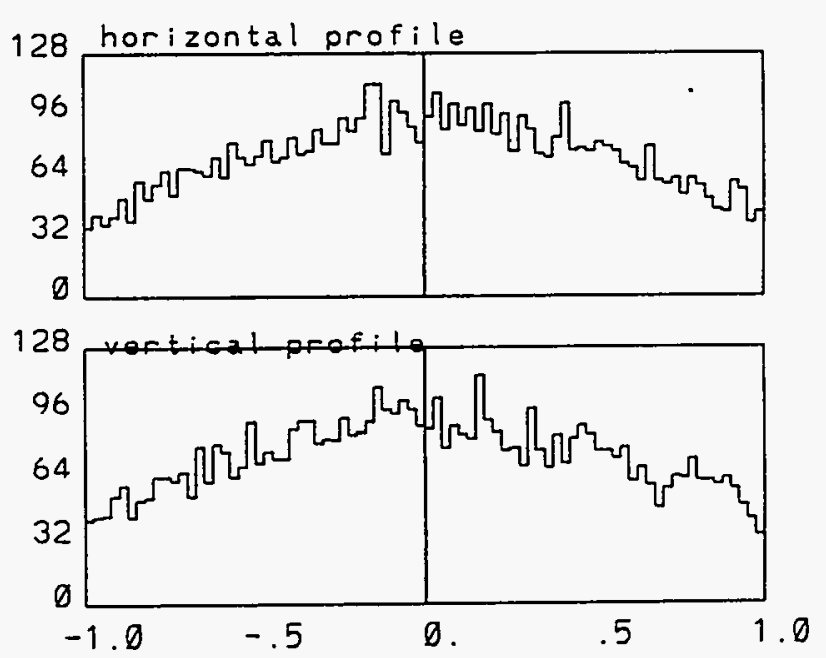
Figure 12-9 shows the beam profile at position $(n e=30) z=70$, with the Inline-Injection system of Solenoid + Gun + Solenoid combination inline with the linac. With Solenoid current of $I=3400$ amp.

- BNL RF GUN

60.0

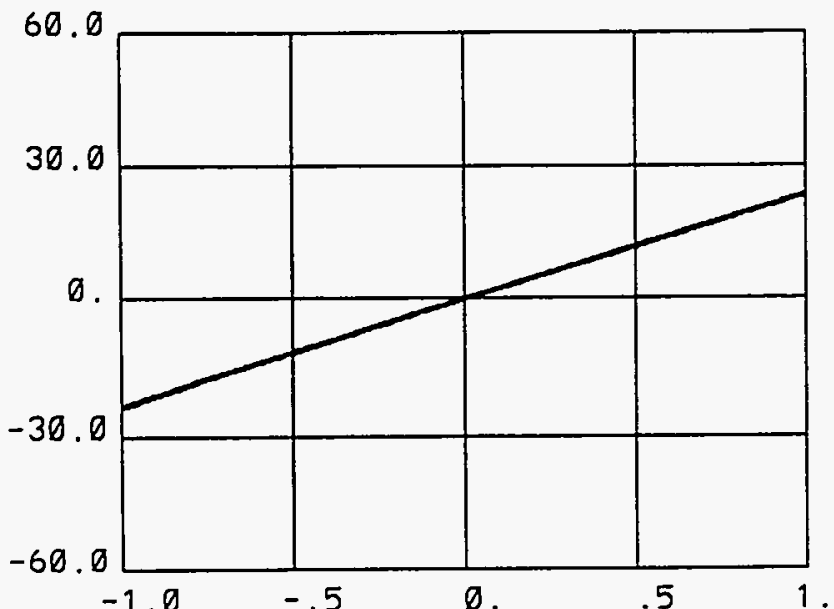

$(0.9 \mathrm{~mm}), 5.15 \mathrm{ps}, 100 \mathrm{MV} / \mathrm{m}, 1 \mathrm{nC}, \mathrm{phi}=43.01$.

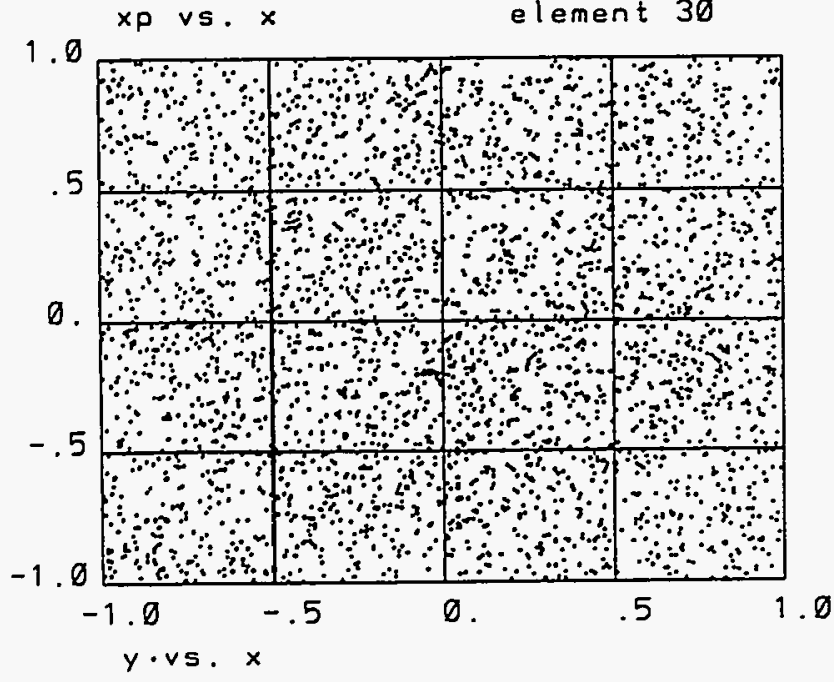

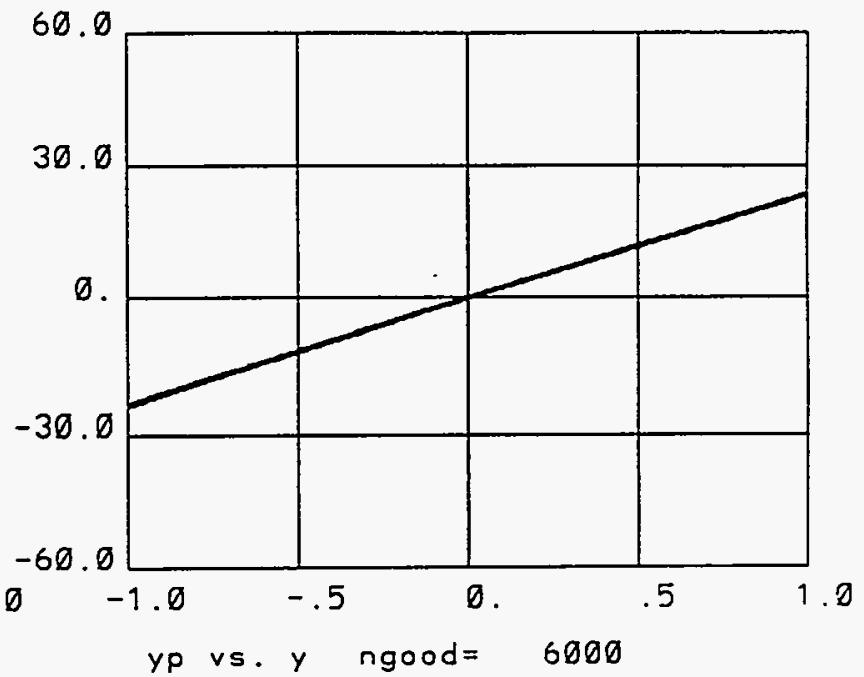

60.0
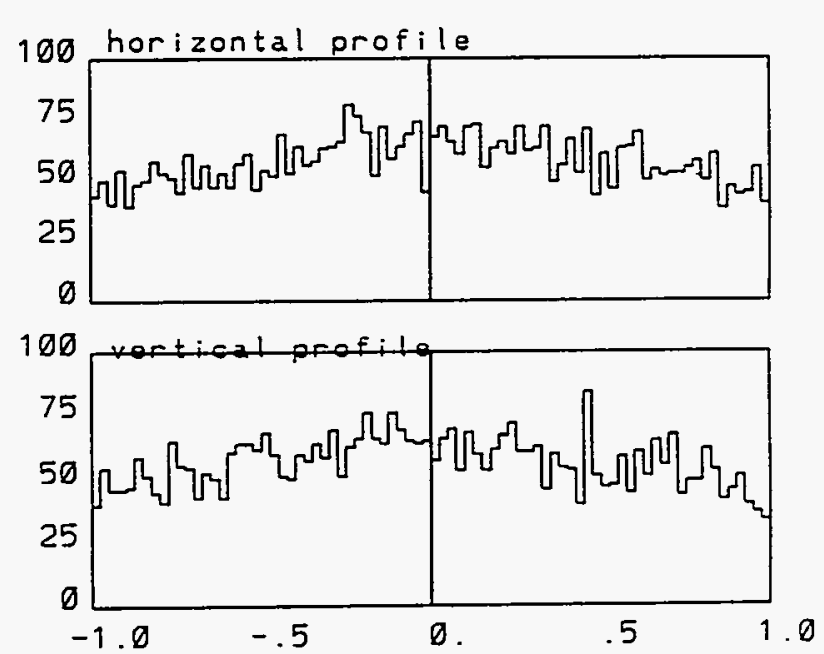
Figure 12-10 shows the beam profile at position $(n e=35) \quad z=95$, with the Inline-Injection system of Solenoid + Gun + Solenoid combination inline with the linac. With Solenoid current of $I=3400 \mathrm{amp}$.

- BNL RF GUN

60.0
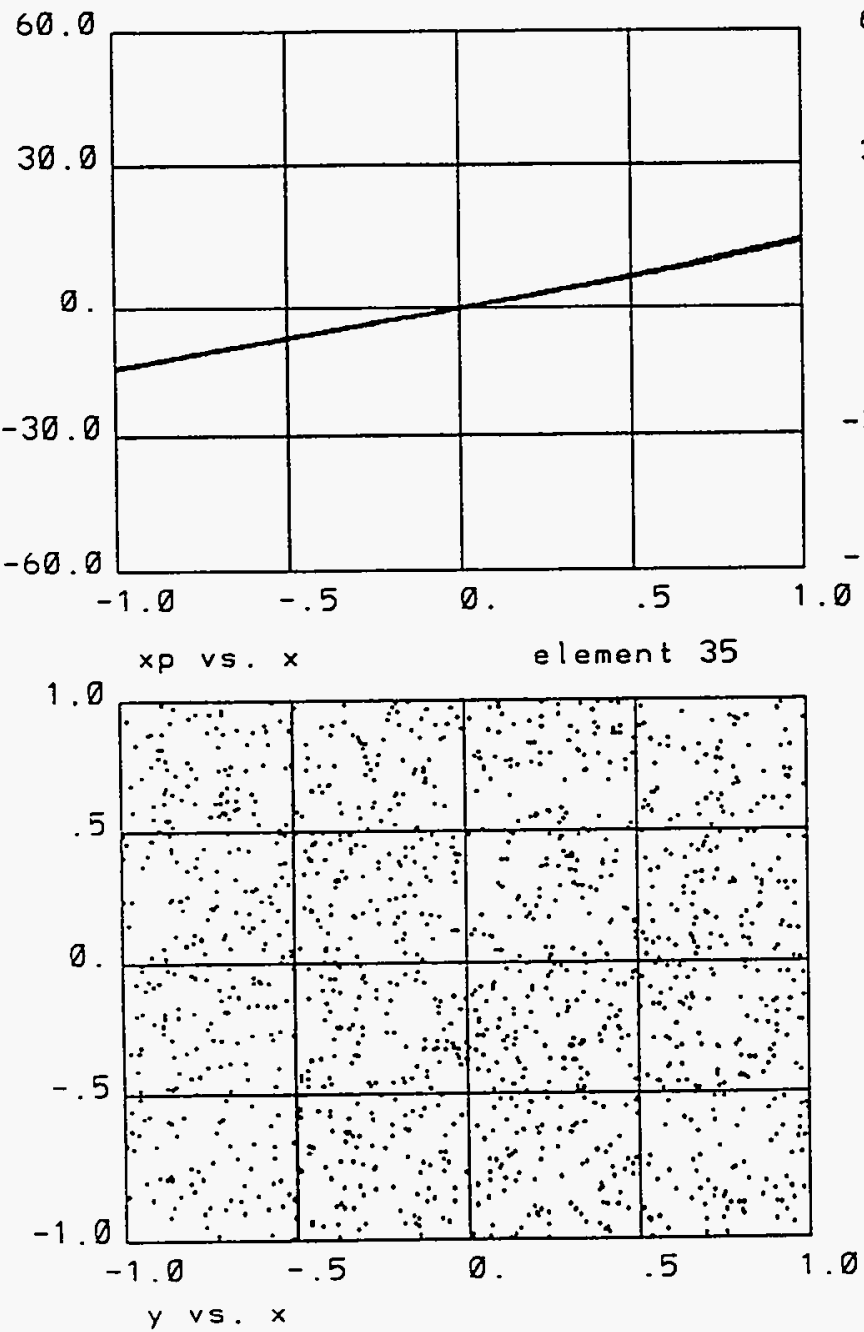

$(0.9 \mathrm{~mm}), 5.15 p s .100 M V / m, 1 \mathrm{nC}, \mathrm{phi}=43.0)^{\circ}$
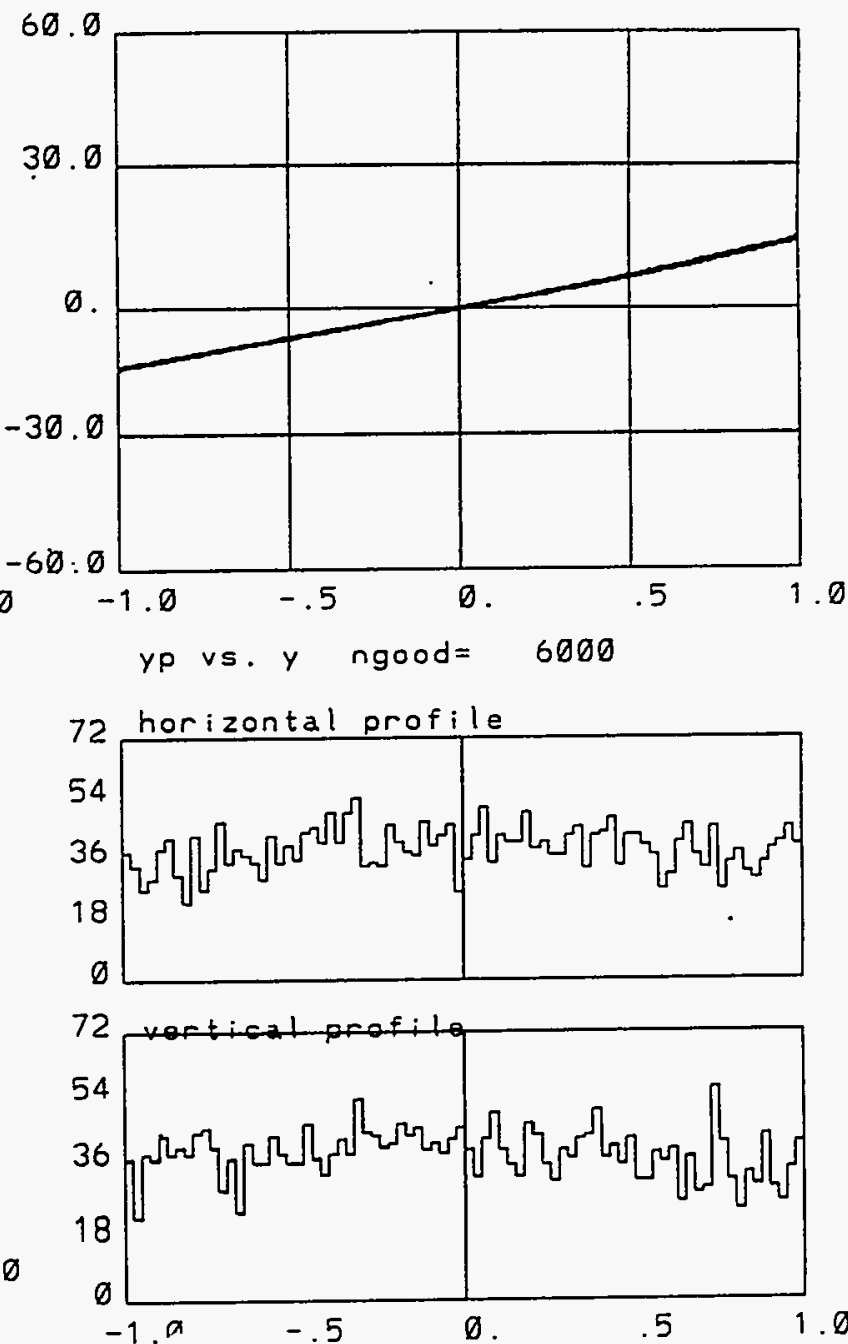
Figures $13-1$ to 13-10 shows the beam profile at various positions (distance $\mathbf{z}$ from the cathode), with the Inline-Injection system of Solenoid + Gun + Solenoid combination inline with the linac. With Solenoid current of $I=3800 \mathrm{amp}$.

'BNL RF GUN

60.0

30.8

$\emptyset$.

$-30.0$
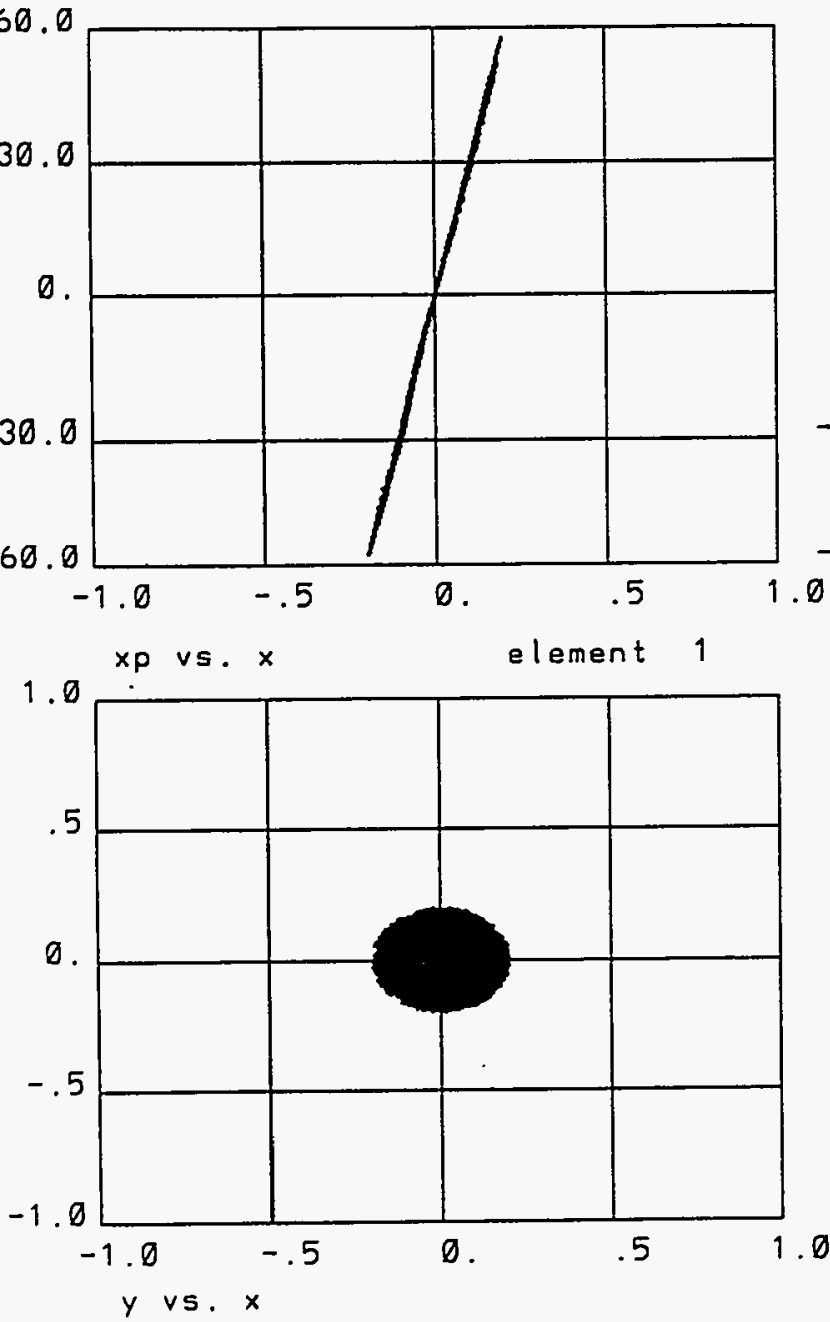

$(0.9 \mathrm{~mm}), 5.15 \mathrm{ps}, 100 \mathrm{MV} / \mathrm{m}, 1 \mathrm{nC}, \mathrm{phi}=43.0)$.
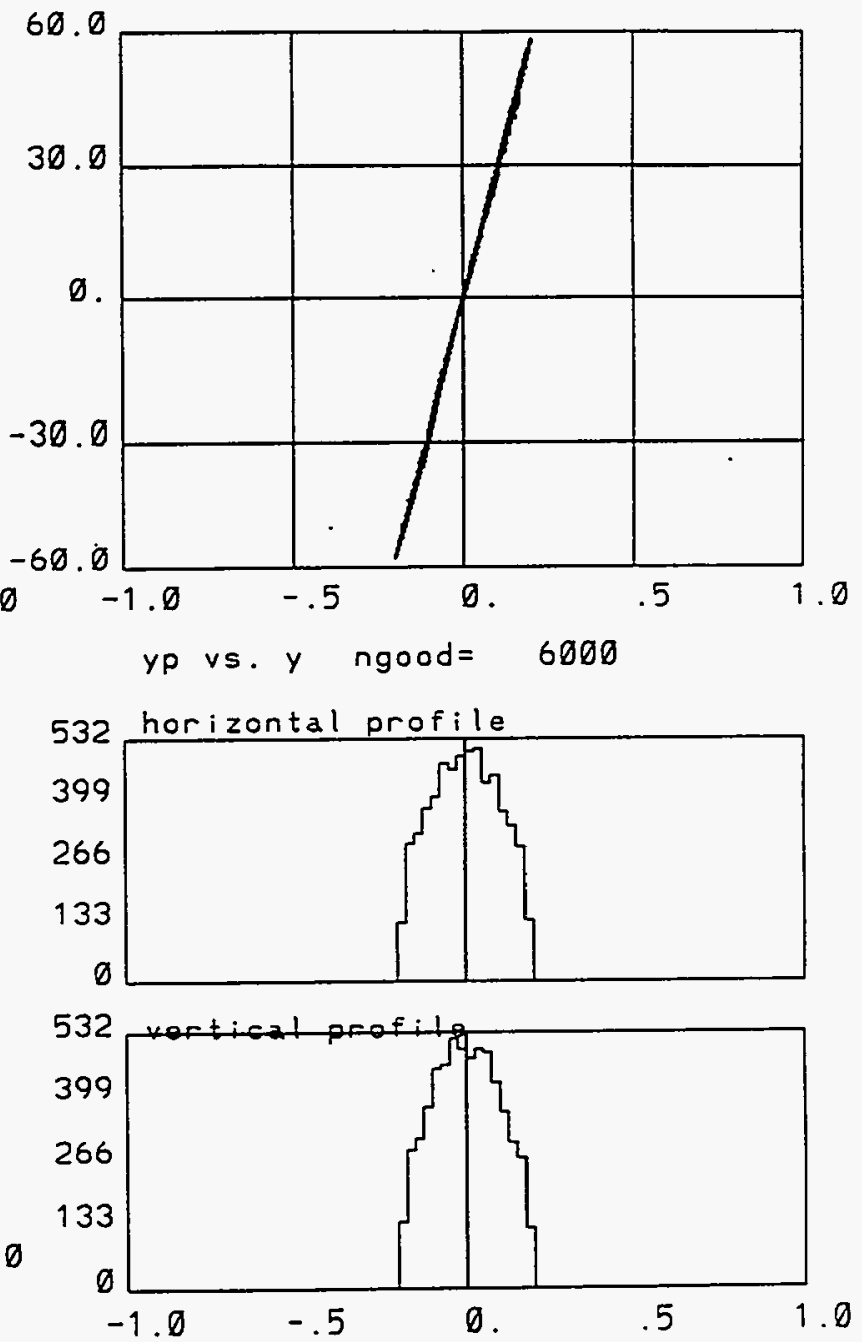

Figure $13-1$ shows the beam profile at position $(n e=1) z=0$, with the Inline-Injection system of Solenoid + Gun + Solenoid combination inline with the linac. With Solenoid current of $I=3800$ amp. 
Figure $13-2$ shows the beam profile at position $(n e=2) z=2.6$, with the Inline-Injection system of Solenoid + Gun + Solenoid combination inline with the linac. With Solenoid current of $I=3800$ amp.

- BNL RF GUN

$$
60 .
$$
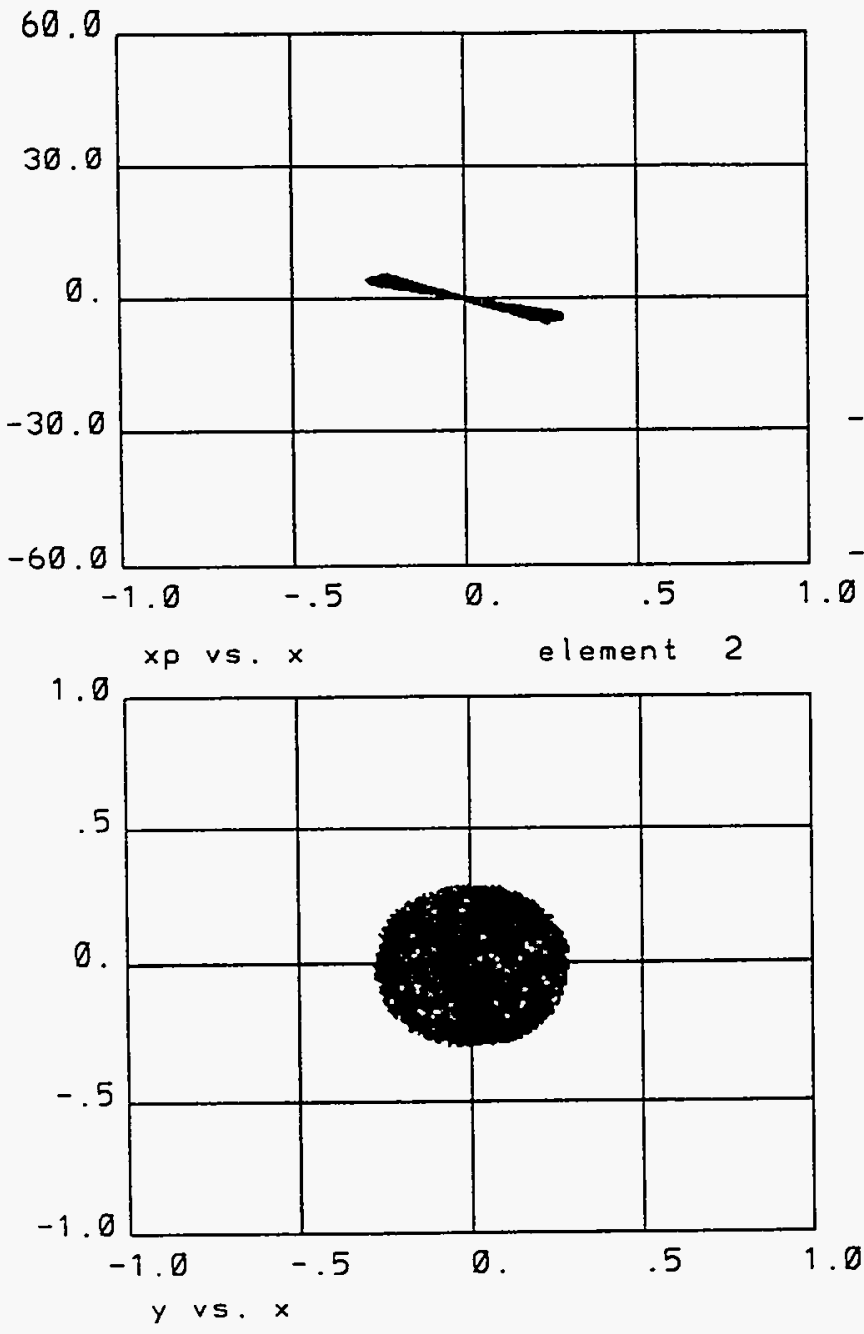

$(0.9 \mathrm{~mm}), 5.15 p s, 100 \mathrm{MV} / \mathrm{m}, 1 \mathrm{nC}, \mathrm{phi}=43.0)^{\circ}$
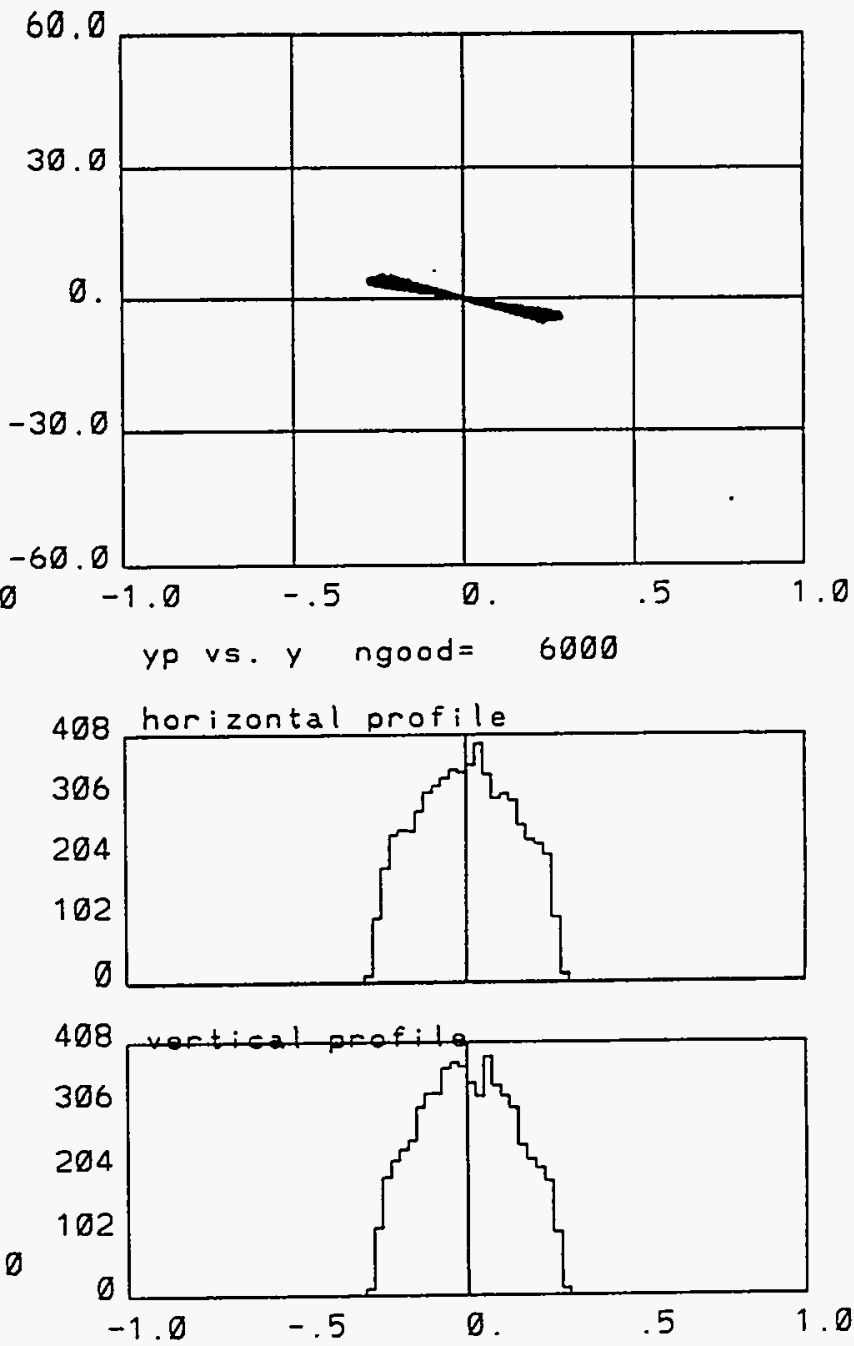
Figure $13-3$ shows the beam profile at position (ne=3) $z=5.2$, with the Inline-Injection system of Solenoid + Gun + Solenoid combination inline with the linac. With Solenoid current of $I=3800 \mathrm{amp}$.

'BNL RF GUN
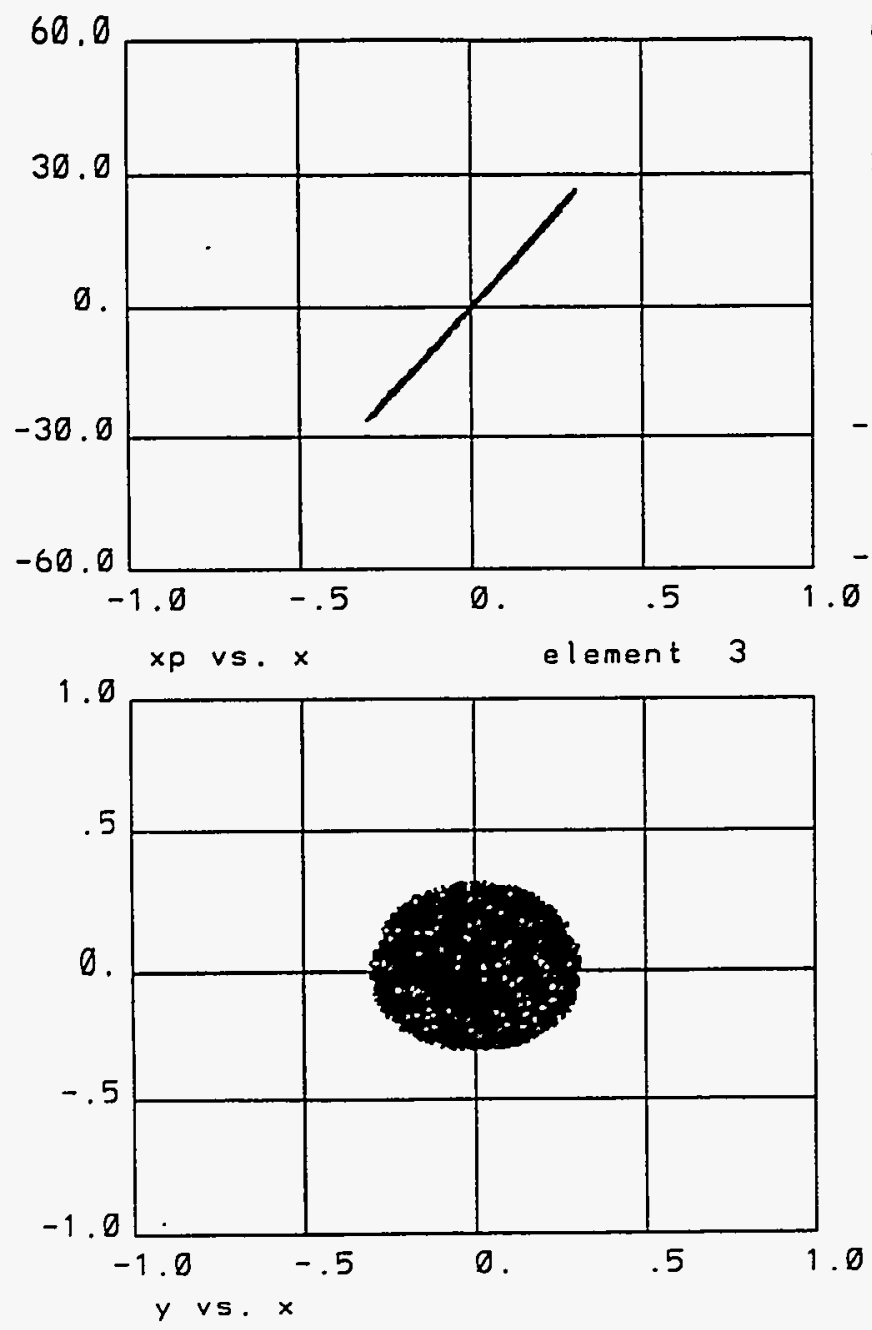

$(0.9 \mathrm{~mm}), 5.15 \mathrm{ps}, 100 \mathrm{MV} / \mathrm{m}, 1 \mathrm{nC}, \mathrm{phi}=43.0)$.
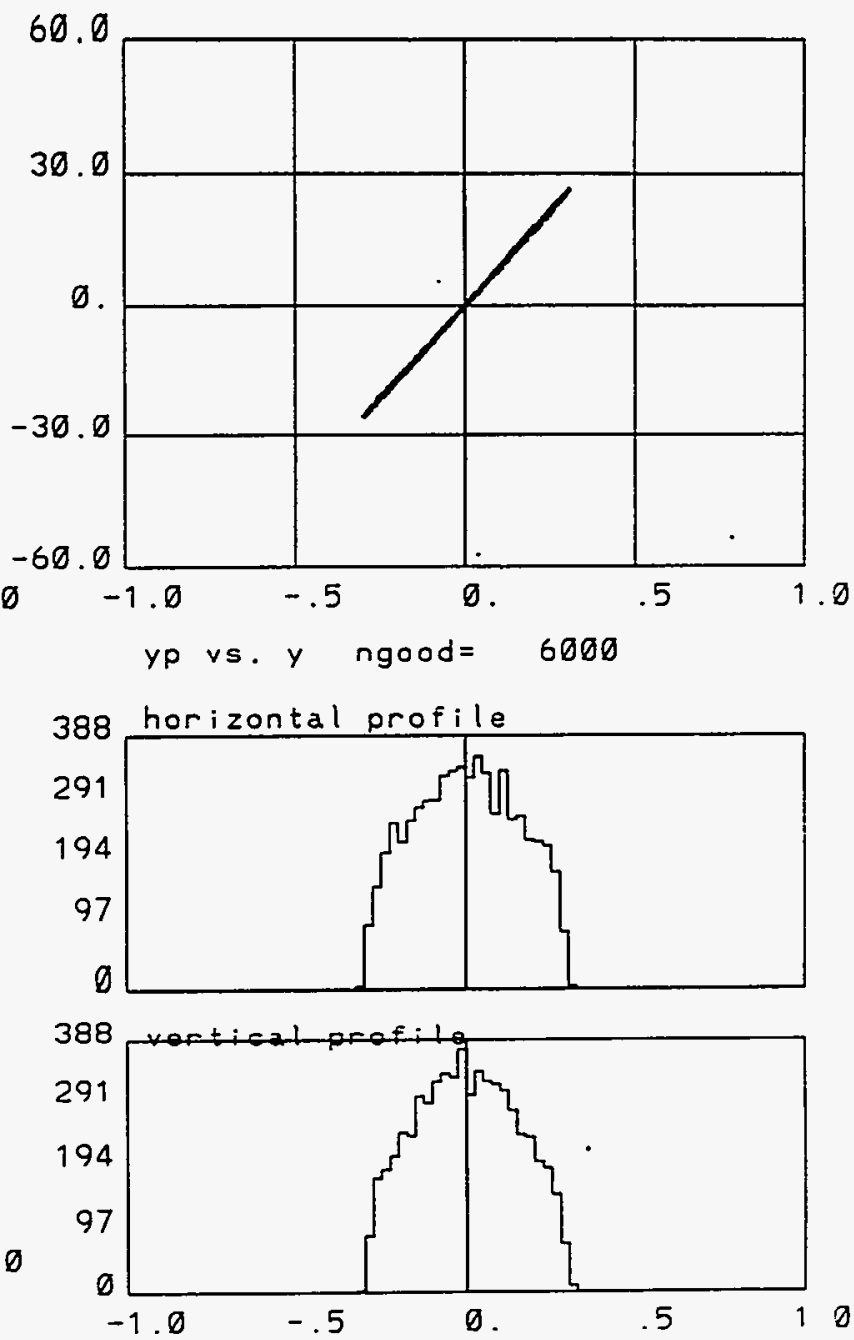
Figure 13-4 shows the beam profile at position $(n e=4) z=7.9$, with the Inline-Injection system of Solenoid + Gun + Solenoid combination inline with the linac. With Solenoid current of $I=3800$ amp.

- BNL RF GUN
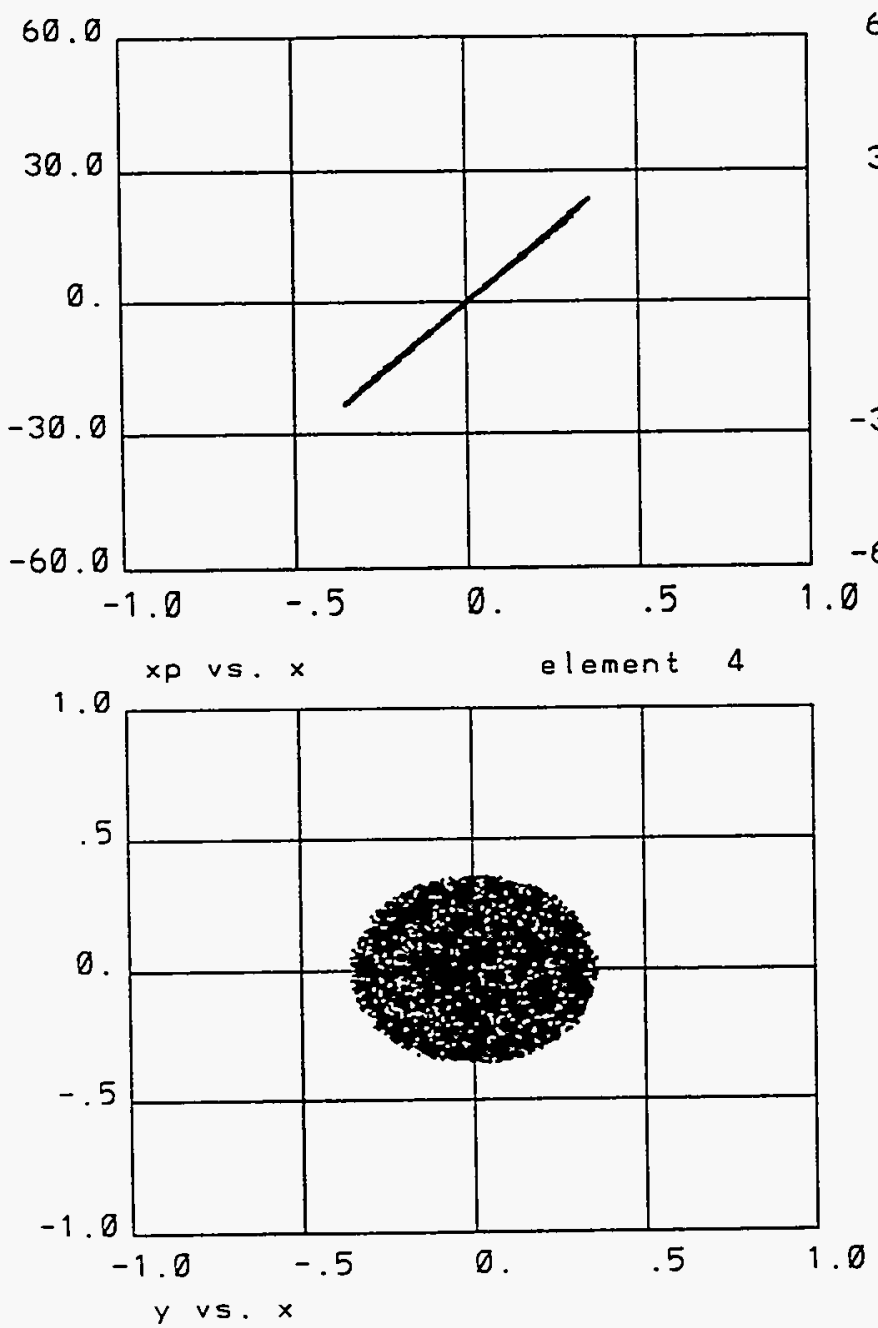

$(0.9 \mathrm{~mm}), 5.15 \mathrm{ps}, 100 \mathrm{MV} / \mathrm{m}, 1 \mathrm{nC}, \mathrm{phi}=43.0)^{\circ}$

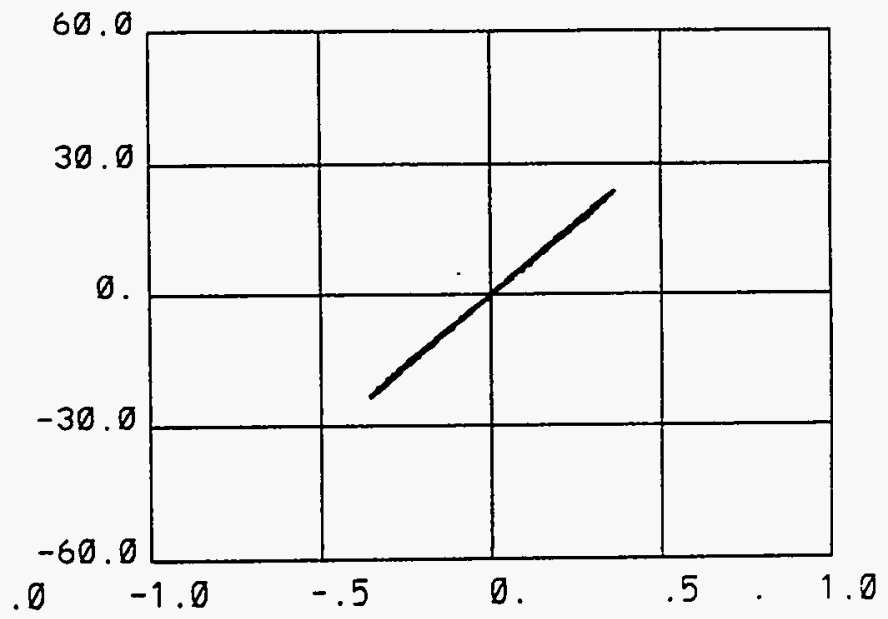

yp vs. y ngood $=6000$
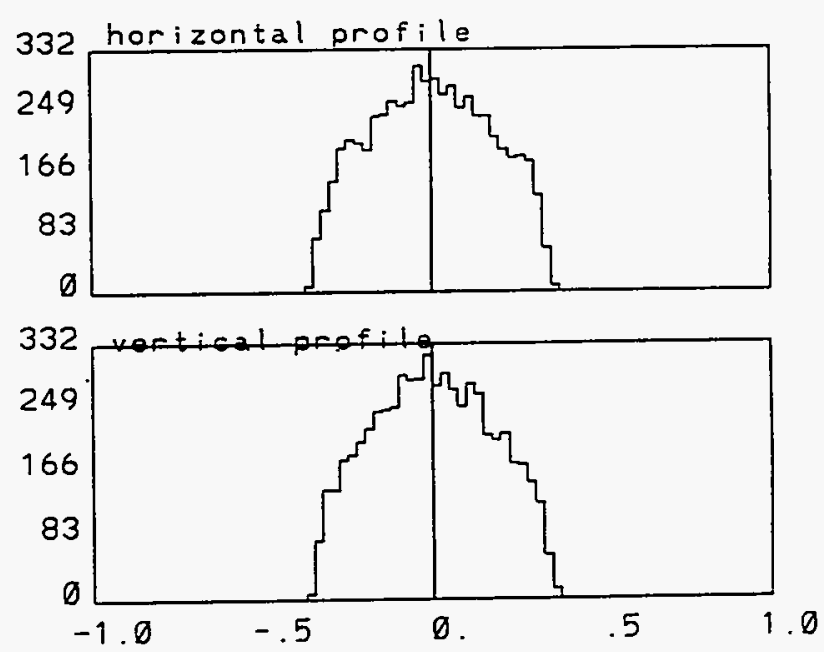
Figure $13-5$ shows the beam profile at position $(n e=5) z=10$, with the Inline-Injection system of Solenoid + Gun + Solenoid combination inline with the linac. With Solenoid current of $I=3800$ amp.

- BNL RF GUN

$$
60.0
$$
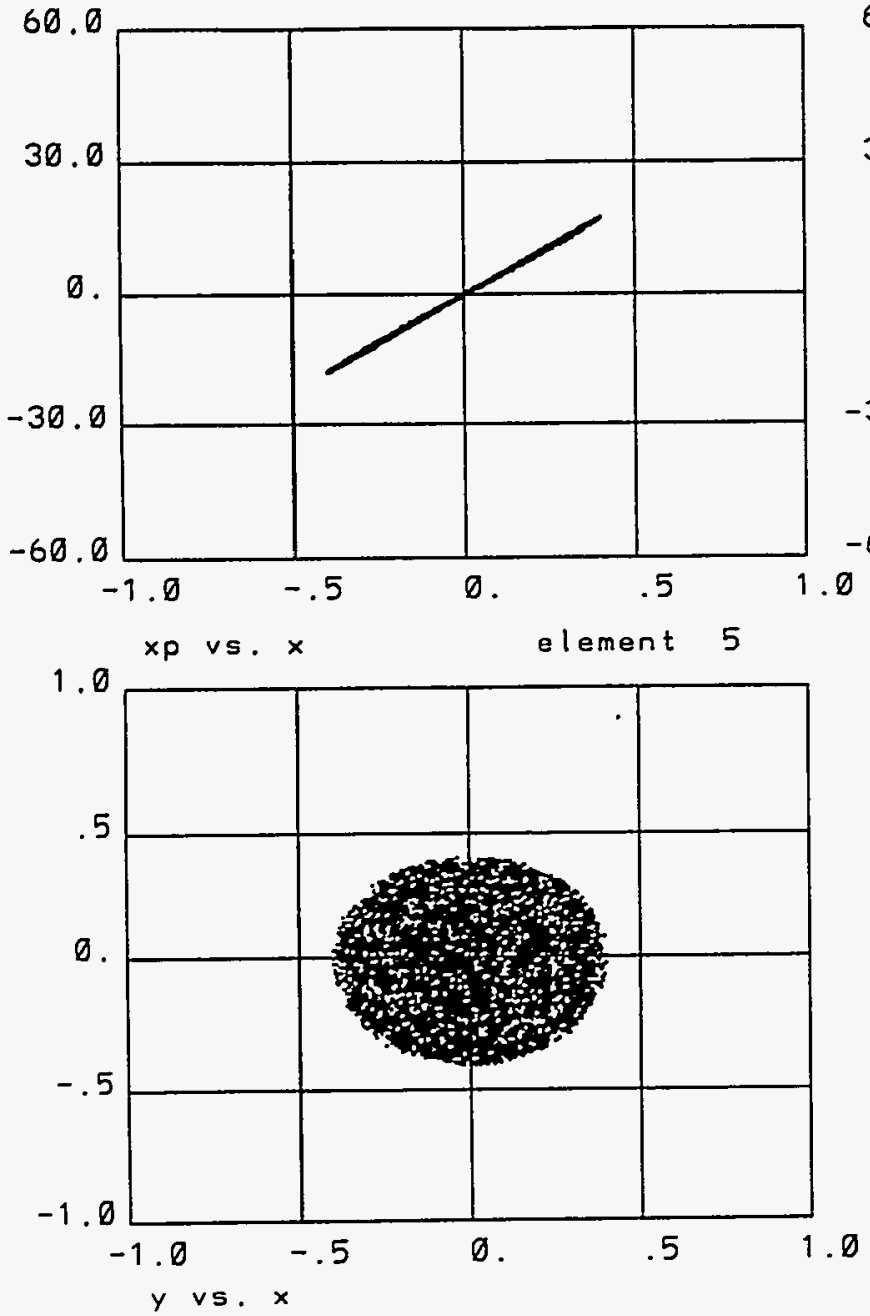

$(0.9 \mathrm{~mm}), 5.15 \mathrm{ps}, 100 \mathrm{MV} / \mathrm{m}, 1 \mathrm{nC}$, phi $=43.0)^{\circ}$

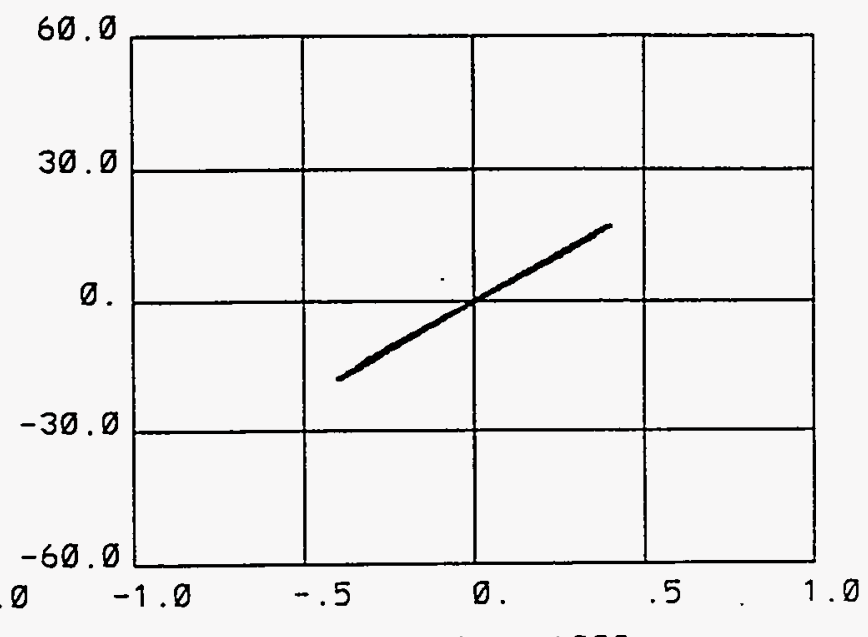

yp vs. y ngood $=6000$
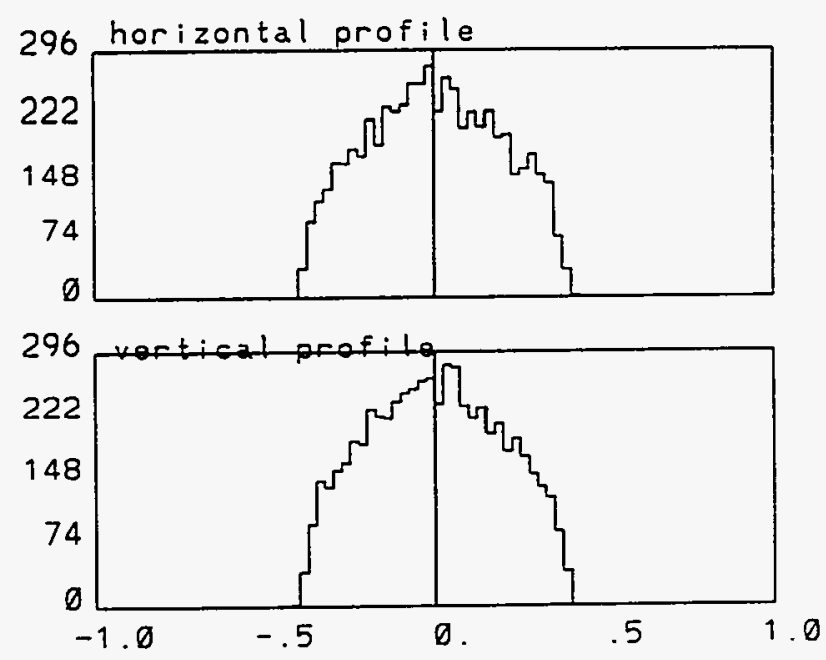
Figure $13-6$ shows the beam profile at position $(n e=10) z=20$, with the Inline-Injection system of Solenoid + Gun + Solenoid combination inline with the linac. With Solenoid current of $I=3800$ amp.

-BNL RF GUN
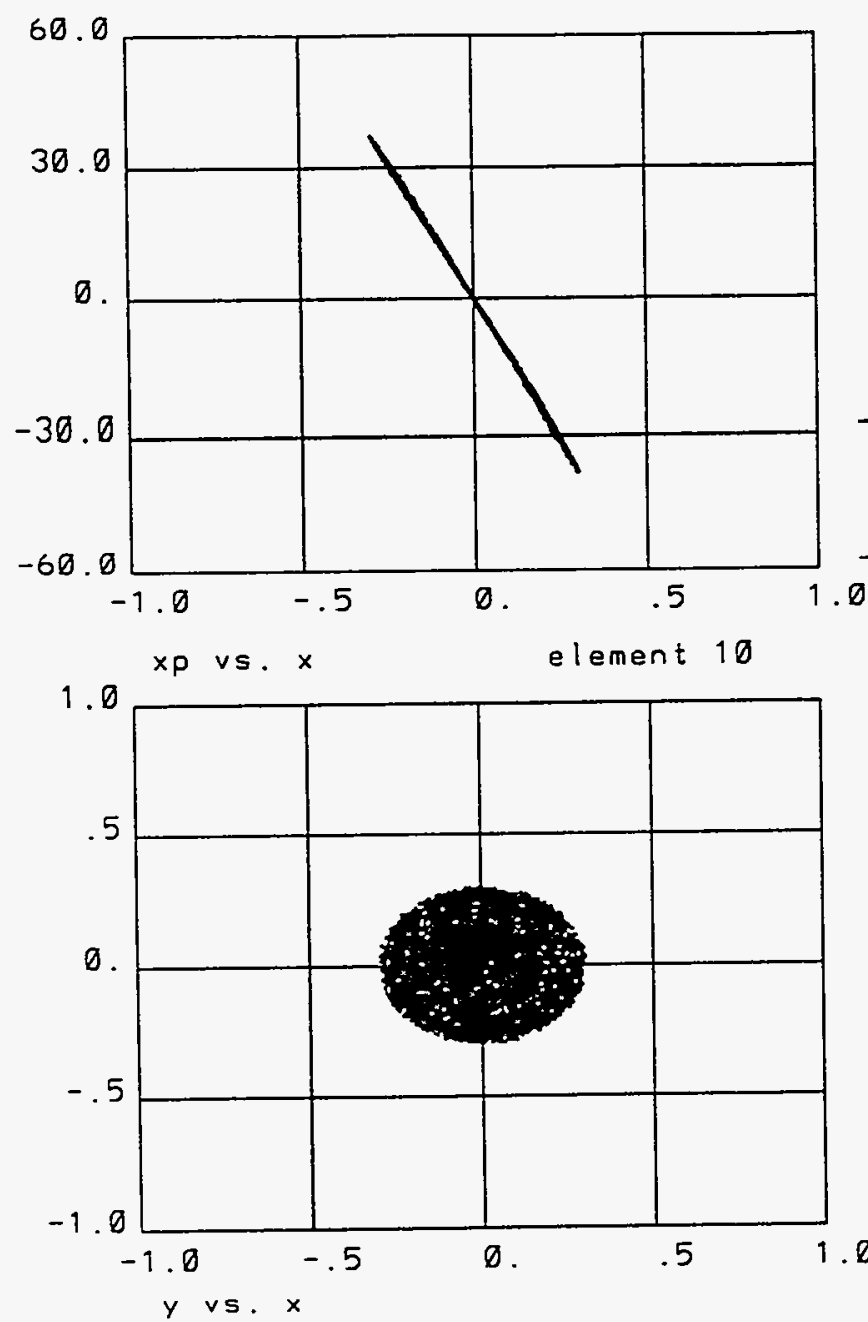

$(0.9 \mathrm{~mm}), 5.15 \mathrm{ps}, 100 \mathrm{MV} / \mathrm{m} .1 \mathrm{nC}, \mathrm{phi}=43.0)^{\circ}$

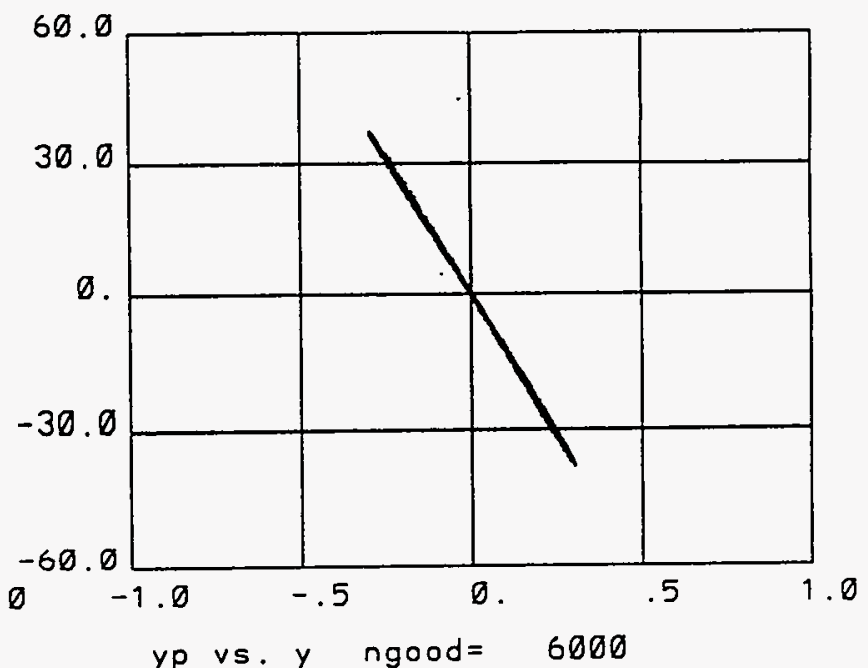

388 horizontal profile
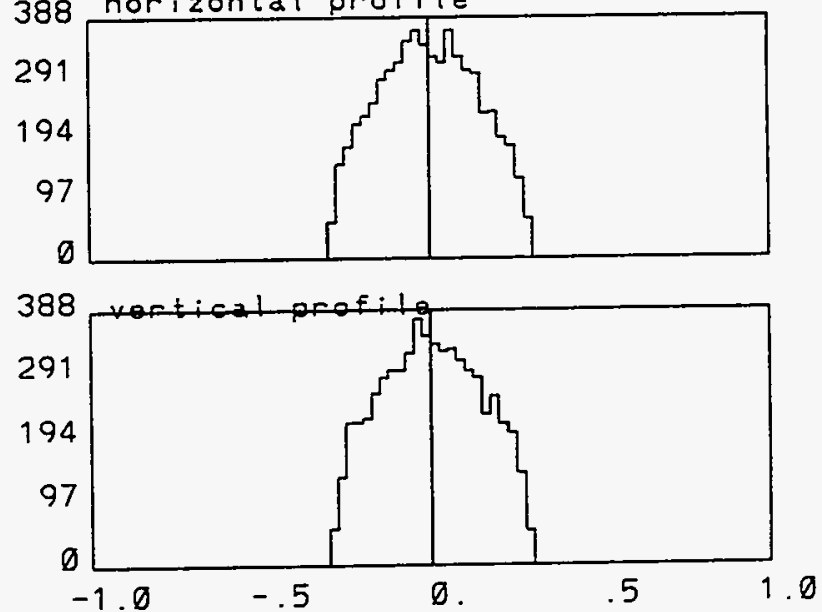
Figure $13-7$ shows the beam profile at position $(n e=20) z=40$, with the Inline-Injection system of Solenoid + Gun + Solenoid combination inline with the linac. With Solenoid current of $\mathrm{I}=\mathbf{3 8 0 0} \mathrm{amp}$.

'BNL RF GUN
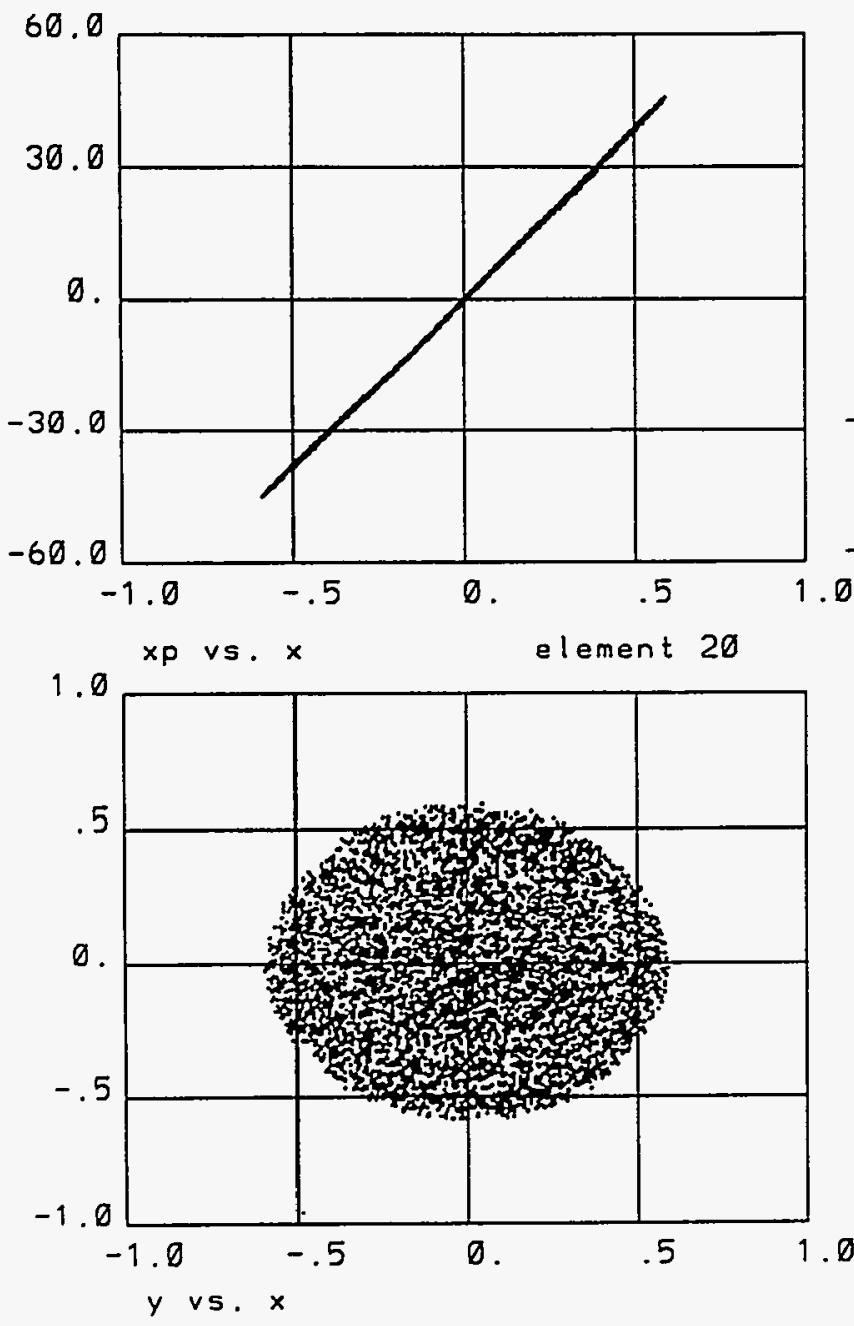

$(0.9 \mathrm{~mm}), 5.15 p s, 100 \mathrm{MV} / \mathrm{m}, 1 \mathrm{nC}, \mathrm{phi}=43.0)^{\prime}$

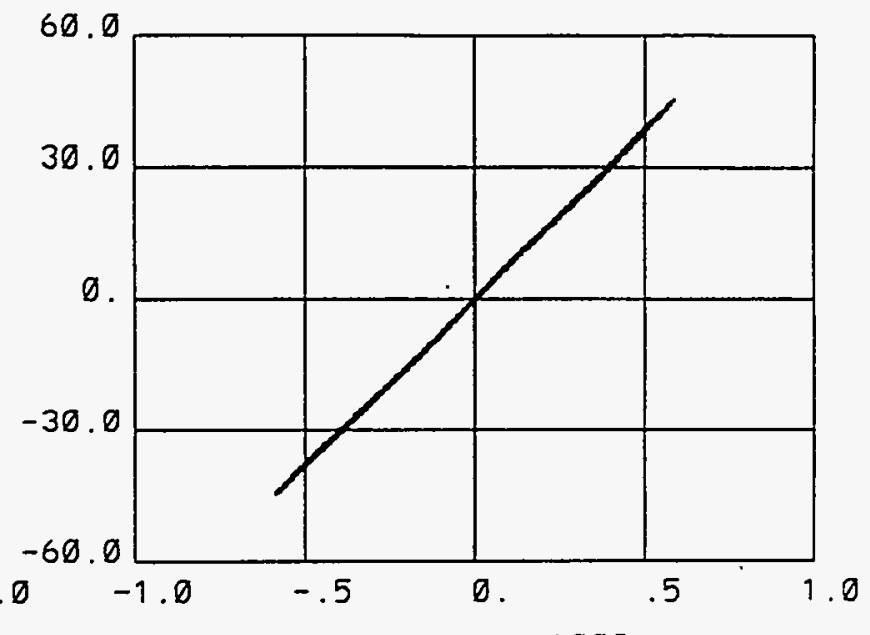

yp vs. y ngood $=6000$
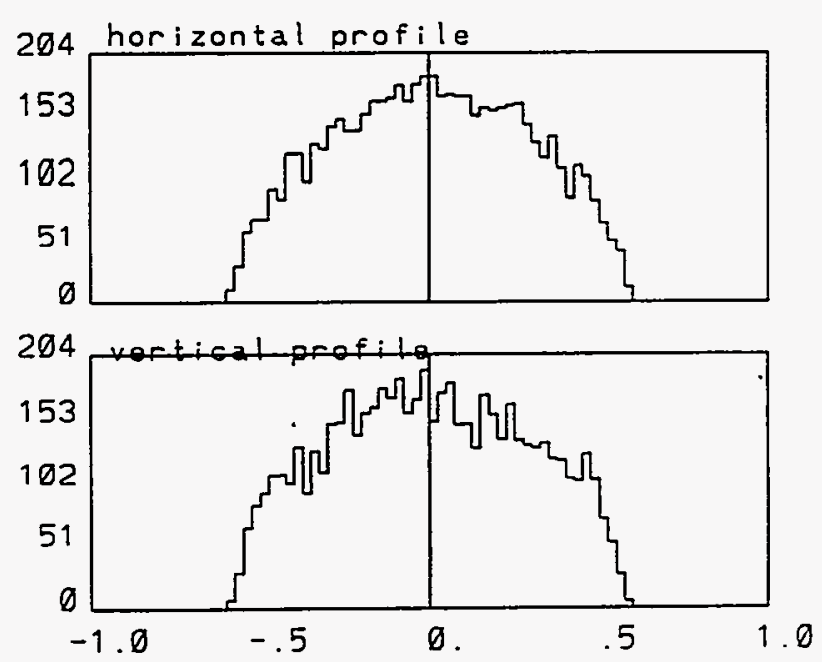
Figure 13-8 shows the beam profile at position $(n e=27) z=58$, with the Inline-Injection system of Solenoid + Gun + Solenoid combination inline with the linac. With Solenoid current of $\mathrm{I}=3800 \mathrm{amp}$.
-BNL RF GUN
$(0.9 \mathrm{~mm}): 5.15 \mathrm{ps} .100 \mathrm{MV} / \mathrm{m}, 1 \mathrm{nC}, \mathrm{phi}=43.0)$
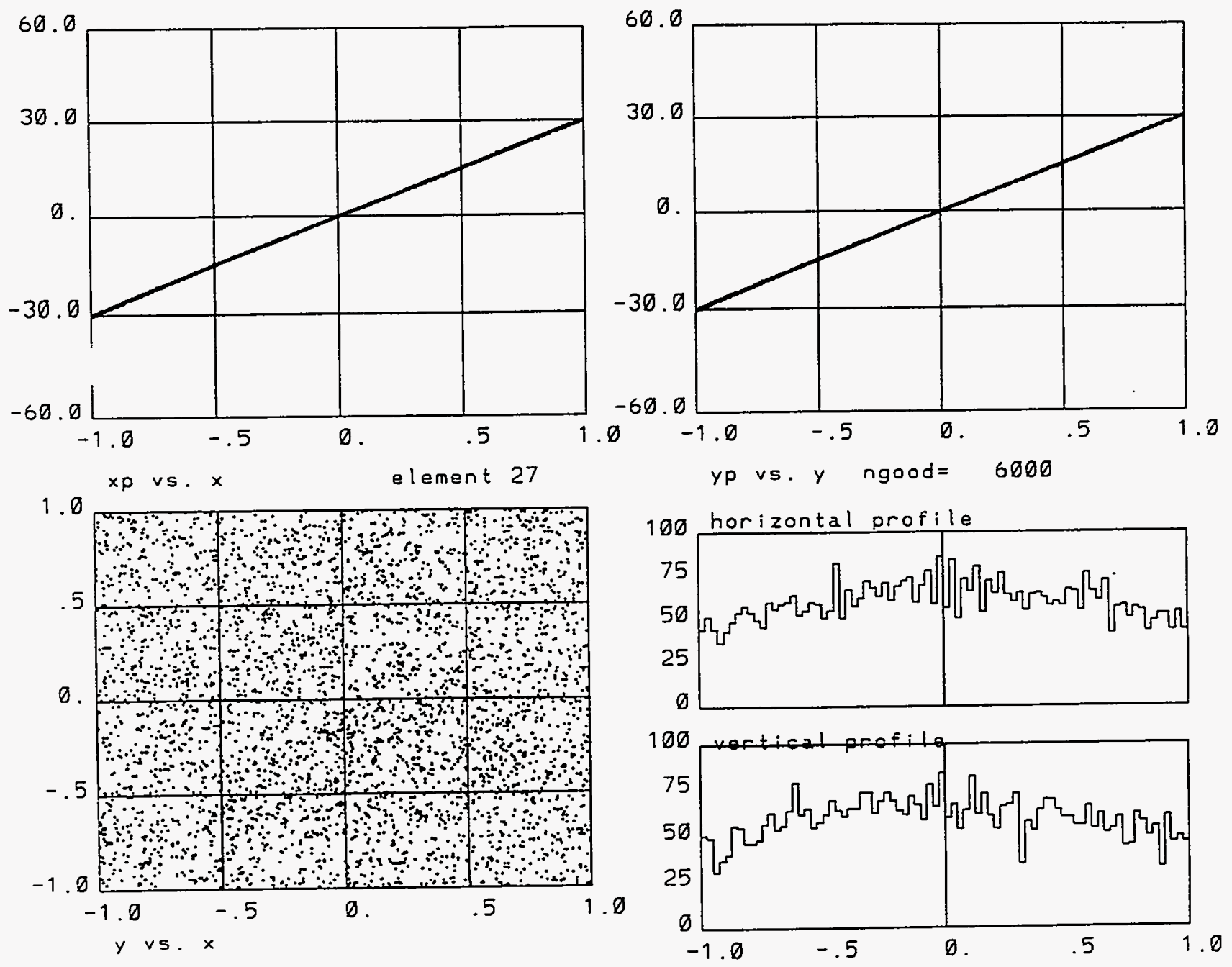
Figure 13-9 shows the beam profile at position $(n e=30) z=70$, with the Inline-Injection system of Solenoid + Gun + Solenoid combination inline with the linac. With Solenoid current of $I=3800 \mathrm{amp}$.

- BNL RF GUN
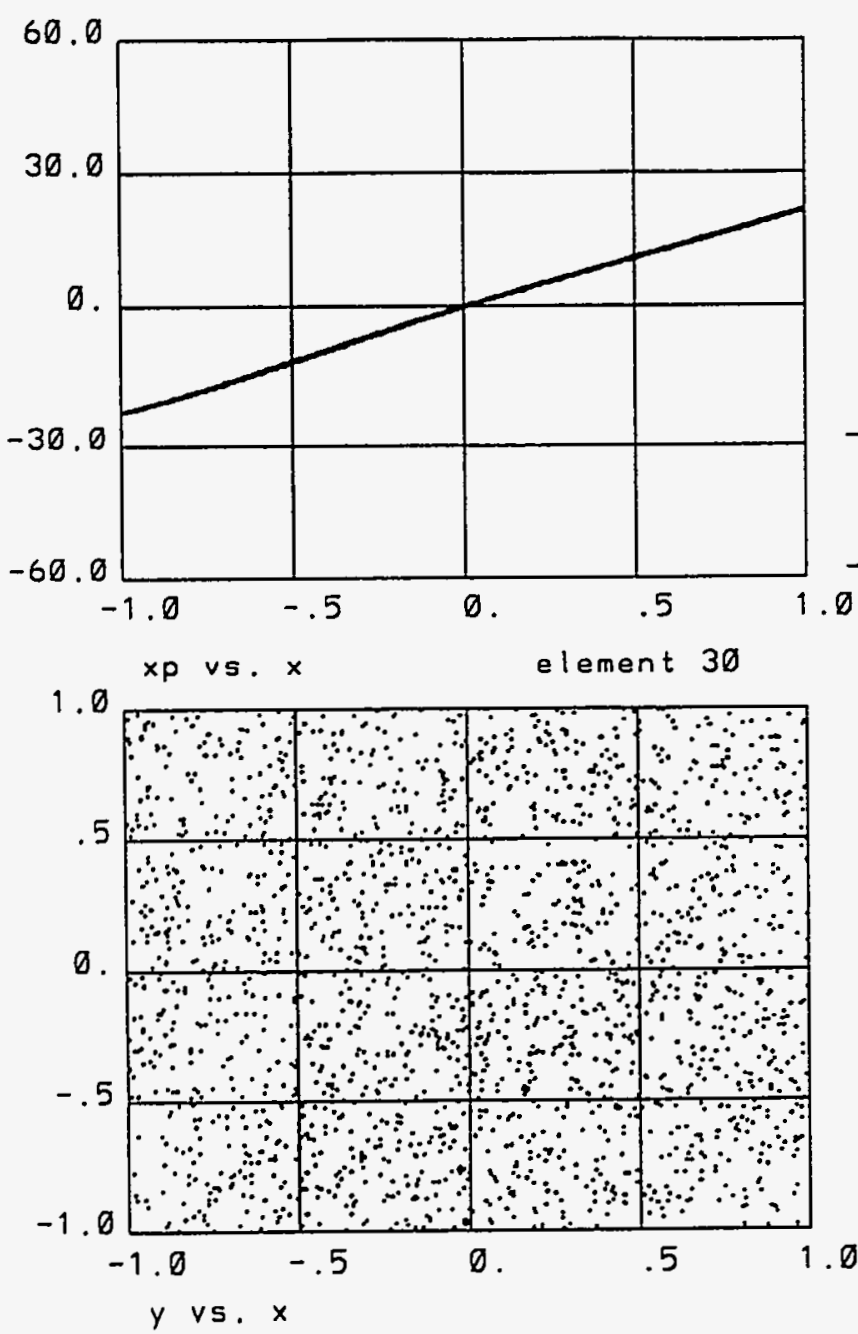

$(0.9 \mathrm{~mm}), 5.15 \mathrm{ps}, 100 \mathrm{MV} / \mathrm{m}, 1 \mathrm{nC}, \mathrm{phi}=43.01$.
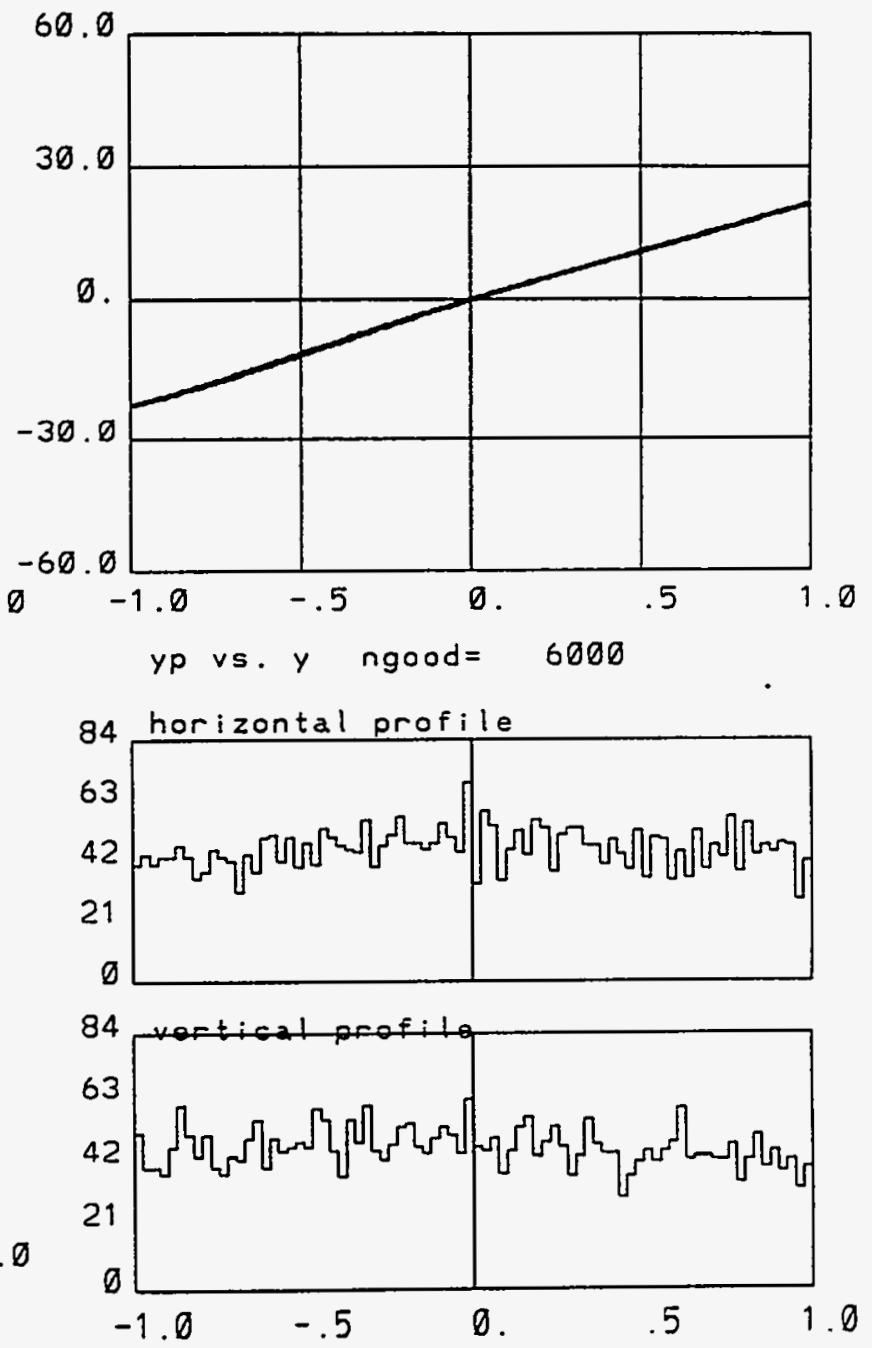
Figure 13-10 shows the beam profile at position $(n e=35) \quad z=95$, with the Inline-Injection system of Solenoid + Gun + Solenoid combination inline with the linac. With Solenoid current of $I=3800$ amp.

'BNL RF GUN $\quad,(0.9 \mathrm{~mm}), 5.15 \mathrm{ps}, 100 \mathrm{MV} / \mathrm{m}, 1 \mathrm{nC}, \mathrm{phi}=43 . \emptyset)^{\circ}$
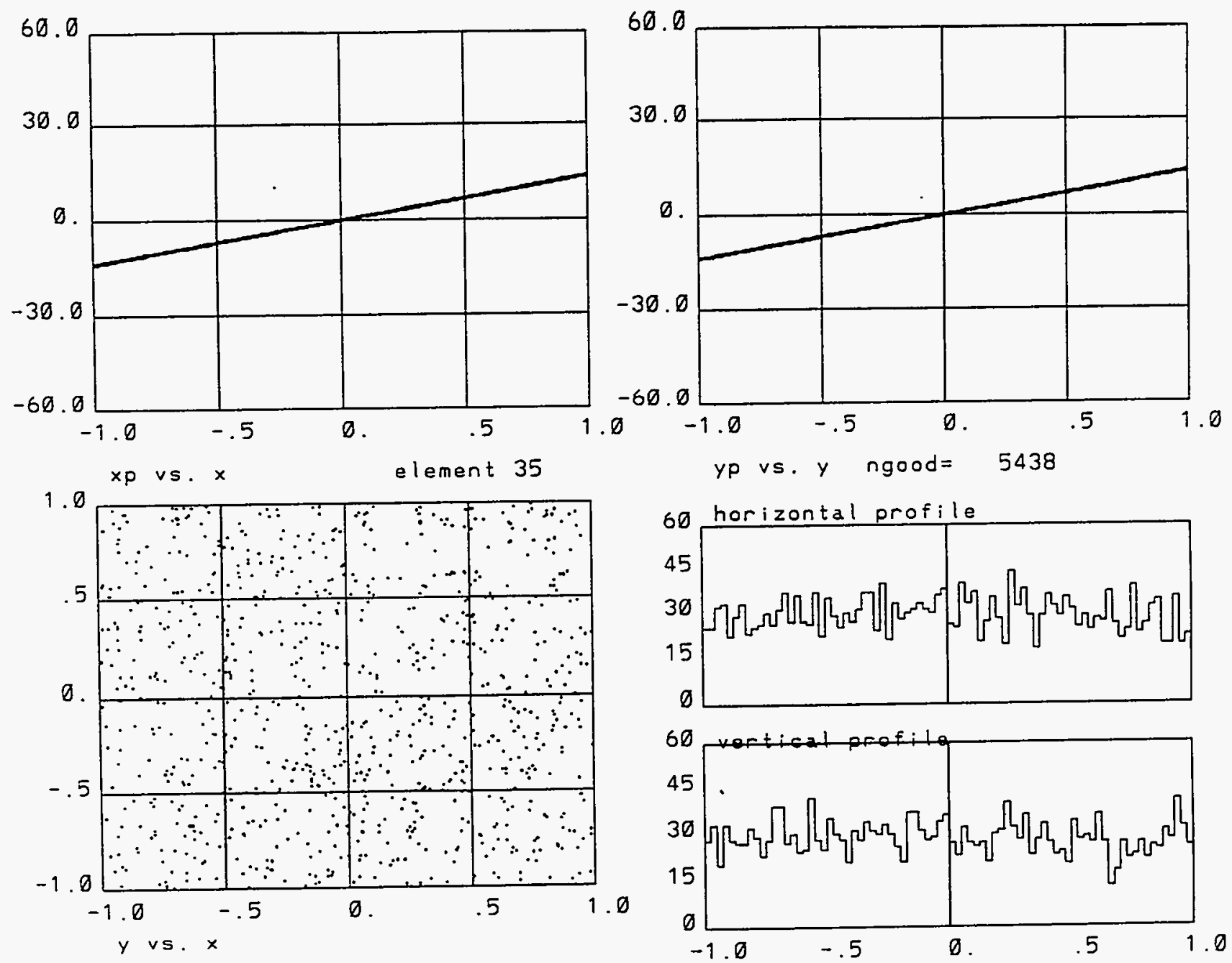


\section{TABLE CAPTIONS:}

Table 1. Shows the labling of the elements in the beam line (in PARMELA format) from the Cathode (at $z=0$ ), through the $R F$ Gun and at various points (distance $\mathrm{z}$ from the cathode) to the Linac entrance.

Table 2-1. Shows variation of emittance through the gun, at the the gun exit and out side of the gun through the drift space(s) to the Linac entrance. With $66 \mathrm{MV} / \mathrm{m}$ field on the cathode for the Inline-Injection system with $\underline{\mathrm{I}=0 \text {, and }}$ "flat" beam.

Table 2-2. Shows variation of emittance through the gun, at the the gun exit and out side of the gun through the drift space(s) to the Linac entrance. With $66 \mathrm{MV} / \mathrm{m}$ field on the cathode for the Inline-Injection system with $\underline{\mathrm{I}=0 \text {, }}$ "Gaussian distribution, and $1 \sigma$ cut.

Table 2-3. Shows variation of emittance through the gun, at the the gun exit and out side of the gun through the drift space(s) to the Linac entrance. With $66 \mathrm{MV} / \mathrm{m}$ field on the cathode for the Inline-Injection system with $\underline{\mathrm{I}}=0$, "Gaussian distribution, and $2 \sigma$ cut.

Table 2-4. Shows variation of emittance through the gun, at the the gun exit and out side of the gun through the drift space(s) to the Linac entrance. With $66 \mathrm{MV} / \mathrm{m}$ field on the cathode for the Inline-Injection system with $\underline{\mathrm{I}=0 \text {, }}$ "Gaussian distribution, and $\underline{3 \sigma \text { cut }}$.

Table 3-1. Shows variation of emittance through the gun, at the the gun exit and out side of the gun through the drift space(s) to the Linac entrance. With $66 \mathrm{MV} / \mathrm{m}$ field on the cathode for the Inline-Injection system, solenoid current of $\mathrm{I=2180}$, with a "flat" beam.

Table 3-2. Shows variation of emittance through the gun, at the the gun exit and out side of the gun through the drift space(s) to the Linac entrance. With $66 \mathrm{MV} / \mathrm{m}$ field on the cathode for the Inline-Injection system, solenoid current of $\underline{\mathrm{I}=2180}$, with a "Gaussian distribution, and $1 \sigma$ cut .

Table 3-3. Shows variation of emittance through the gun, at the the gun exit and out side of the gun through the drift space(s) to the Linac entrance. With $66 \mathrm{MV} / \mathrm{m}$ field on the cathode for the Inline-Injection system with solenoid current $\mathrm{I}=2180, \mathrm{a}$ "Gaussian distribution, and $\underline{3 \sigma \text { cut }}$. 


\section{FIGURE CAPTIONS:}

Figure 1. A sketch of the Brookhaven ATF (original design) Injection system with the transport beamline from the gun to the linac entrance. Showing the positions of the magnetic elements.

Figure 2. A sketch of the Brookhaven ATF (new design) Inline-Injection system with the Solenoid + Gun + Solenoid combination placed inline with the linac.

Figures 3-1 to 3-8 shows the beam profile at various positions (distance $\mathrm{z}$ from the cathode), with the Inline-Injection system of Solenoid + Gun + Solenoid combination inline with the linac. With Solenoid current of $\underline{I=2140}$ amp.

Figure $3-1$ shows the beam profile at position (ne=1) $z=0$, with the InlineInjection system of Solenoid + Gun + Solenoid combination inline with the linac. With Solenoid current of $\mathrm{I}=2140 \mathrm{amp}$.

Figure $3-2$ shows the beam profile at position (ne $=2) z=2.6$, with the Inline-Injection system of Solenoid + Gun + Solenoid combination inline with the linac. With Solenoid current of $\mathrm{I}=2140 \mathrm{amp}$.

Figure $3-3$ shows the beam profile at position (ne=3) $z=5.2$, with the Inline-Injection system of Solenoid + Gun + Solenoid combination inline with the linac. With Solenoid current of $\mathrm{I}=2140 \mathrm{amp}$.

Figure $3-4$ shows the beam profile at position $(n e=4) z=7.9$, with the Inline-Injection system of Solenoid + Gun + Solenoid combination inline with the linac. With Solenoid current of $\mathrm{I}=2140 \mathrm{amp}$.

Figure $3-5$ shows the beam profile at position $(n e=5) \quad z=10$, with the InlineInjection system of Solenoid + Gun + Solenoid combination inline with the linac. With Solenoid current of $\mathrm{I}=2140 \mathrm{amp}$.

Figure $3-6$ shows the beam profile at position $(n e=10) \quad z=20$ with the Inline-Injection system of Solenoid + Gun + Solenoid combination inline with the linac. With Solenoid current of $\mathrm{I}=2140 \mathrm{amp}$. 
Figure $3-7$ shows the beam profile at position $(n e=20) z=40$, with the Inline-Injection system of Solenoid + Gun + Solenoid combination inline with the linac. With Solenoid current of $\mathrm{I}=2140 \mathrm{amp}$.

Figure $3-8$ shows the beam profile at position $(n e=30) z=70$, with the Inline-Injection system of Solenoid + Gun + Solenoid combination inline with the linac. With Solenoid current of $\mathrm{I}=2140 \mathrm{amp}$.

Figures $4-1$ to $4-8$ shows the beam profile at various positions (distance $z$ from the cathode), with the Inline-Injection system of Solenoid + Gun + Solenoid combination inline with the linac. With Solenoid current of $I=2140$ amp.

Figure $4-1$ shows the beam profile at position $(n e=1) \quad z=0$, with the InlineInjection system of Solenoid + Gun + Solenoid combination inline with the linac. With Solenoid current of $I=2160$ amp.

Figure $4-2$ shows the beam profile at position $(n e=2) z=2.6$, with the Inline-Injection system of Solenoid + Gun + Solenoid combination inline with the linac. With Solenoid current of $I=2160 \mathrm{amp}$.

Figure $4-3$ shows the beam profile at position (ne=3) $z=5.2$, with the Inline-Injection system of Solenoid + Gun + Solenoid combination inline with the linac. With Solenoid current of $I=2160 \mathrm{amp}$.

Figure $4-4$ shows the beam profile at position $(n e=4) \quad z=7.9$, with the Inline-Injection system of Solenoid + Gun + Solenoid combination inline with the linac. With Solenoid current of $I=2160$ amp.

Figure $4-5$ shows the beam profile at position $(\mathrm{ne}=5) \mathrm{z}=10$, with the InlineInjection system of Solenoid + Gun + Solenoid combination inline with the linac. With Solenoid current of $\mathrm{I}=2160 \mathrm{amp}$.

Figure 4 -6 shows the beam profile at position (ne=10) $\mathrm{z}=20$ with the Inline-Injection system of Solenoid + Gun + Solenoid combination inline with the linac. With Solenoid current of $I=2160 \mathrm{amp}$.

Figure $4-7$ shows the beam profile at position $(\mathrm{ne}=20) \mathrm{z}=40$, with the Inli." -Injection system of Solenoid + Gun + Solenoid combination inline with the linac. With Solenoid current of $\mathrm{I}=2160 \mathrm{amp}$.

Figure $4-8$ shows the beam profile at position $(n e=30) z=70$, with the Inline-Injection system of Solenoid + Gun + Solenoid combination inline with the linac. With Solenoid current of $\mathrm{I}=2160 \mathrm{amp}$. 
Figures $5-1$ to 5-8 shows the beam profile at various positions (distance $z$ from the cathode), with the Inline-Injection system of Solenoid + Gun + Solenoid combination inline with the linac. With Solenoid current of $I=2180$ amp.

Figure $5-1$ shows the beam profile at position $(n e=1) z=0$, with the InlineInjection system of Solenoid + Gun + Solenoid combination inline with the linac. With Solenoid current of $\mathrm{I}=2180 \mathrm{amp}$.

Figure $5-2$ shows the beam profile at position $(n e=2) z=2.6$, with the Inline-Injection system of Solenoid + Gun + Solenoid combination inline with the linac. With Solenoid current of $I=2180 \mathrm{amp}$.

Figure $5-3$ shows the beam profile at position $(n e=3) z=5.2$, with the Inline-Injection system of Solenoid + Gun + Solenoid combination inline with the linac. With Solenoid current of $I=2180 \mathrm{amp}$.

Figure $5-4$ shows the beam profile at position $(n e=4) z=7.9$, with the Inline-Injection system of Solenoid + Gun + Solenoid combination inline with the linac. With Solenoid current of $I=2180 \mathrm{amp}$.

Figure $5-5$ shows the beam profile at position $(n e=5) \quad z=10$, with the InlineInjection system of Solenoid + Gun + Solenoid combination inline with the linac. With Solenoid current of $I=2180 \mathrm{amp}$.

Figure $5-6$ shows the beam profile at position $(n e=10) z=20$ with the Inline-Injection system of Solenoid + Gun + Solenoid combination inline with the linac. With Solenoid current of $\mathrm{I}=2180 \mathrm{amp}$.

Figure 5-7 shows the beam profile at position $(\mathrm{ne}=20) \mathrm{z}=40$, with the Inline-Injection system of Solenoid + Gun + Solenoid combination inline with the linac. With Solenoid current of $\mathrm{I}=2180 \mathrm{amp}$.

Figure $5-8$ shows the beam profile at position $(n e=30) z=70$, with the Inline-Injection system of Solenoid + Gun + Solenoid combination inline with the linac. With Solenoid current of $I=2180 \mathrm{amp}$.

Figures $6-1$ to $6-8$ shows the beam profile at various positions (distance $z$ from the cathode), with the Inline-Injection system of Solenoid + Gun + Solenoid combination inline with the linac. With Solenoid current of $I=2200$ amp. 
Figure $6-1$ shows the beam profile at position $(n e=1) z=0$, with the InlineInjection system of Solenoid + Gun + Solenoid combination inline with the linac. With Solenoid current of $I=2200 \mathrm{amp}$.

Figure $6-2$ shows the beam profile at position $(n e=2) \quad z=2.6$, with the Inline-Injection system of Solenoid + Gun + Solenoid combination inline with the linac. With Solenoid current of $\mathrm{I}=2200 \mathrm{amp}$.

Figure $6-3$ shows the beam profile at position (ne $=3$ ) $z=5.2$, with the Inline-Injection system of Solenoid + Gun + Solenoid combination inline with the linac. With Solenoid current of $\mathrm{I}=2200 \mathrm{amp}$.

Figure $6-4$ shows the beam profile at position $(n e=4) z=7.9$, with the Inline-Injection system of Solenoid + Gun + Solenoid combination inline with the linac. With Solenoid current of $\mathrm{I}=2200 \mathrm{amp}$.

Figure $6-5$ shows the beam profile at position $(n e=5) z=10$, with the InlineInjection system of Solenoid + Gun + Solenoid combination inline with the linac. With Solenoid current of $\mathrm{I}=2200 \mathrm{amp}$.

Figure $6-6$ shows the beam profile at position $(n e=10) \quad z=20$ with the Inline-Injection system of Solenoid + Gun + Solenoid combination inline with the linac. With Solenoid current of $\mathrm{I}=2200 \mathrm{amp}$.

Figure $6-7$ shows the beam profile at position $(n e=20) z=40$, with the Inline-Injection system of Solenoid + Gun + Solenoid combination inline with the linac. With Solenoid current of $\mathrm{I}=2200 \mathrm{amp}$.

Figure $6-8$ shows the beam profile at position $(\mathrm{ne}=30) \mathrm{z}=70$, with the Inline-Injection system of Solenoid + Gun + Solenoid combination inline with the linac. With Solenoid current of $\mathrm{I}=2200 \mathrm{amp}$.

Figures $7-1$ to $7-8$ shows the beam profile at various positions (distance $z$ from the cathode), with the Inline-Injection system of Solenoid + Gun + Solenoid combination inline with the linac. With Solenoid current of $I=2400$ amp.

Figure $7-1$ shows the beam profile at position $(n e=1) z=0$, with the InlineInjection system of Solenoid + Gun + Solenoid combination inline with the linac. With Solenoid current of $I=2400 \mathrm{amp}$.

Figure $7-2$ shows the beam profile at position $(\mathrm{ne}=2) \mathrm{z}=2.6$, with the Inline-Injection system of Solenoid + Gun + Solenoid combination inline with the linac. With Solenoid current of $\mathrm{I}=2400 \mathrm{amp}$. 
Figure 7-3 shows the beam profile at position $(n e=3) z=5.2$, with the InlineInjection system of Solenoid + Gun + Solenoid combination inline with the linac. With Solenoid current of $I=2400 \mathrm{amp}$.

Figure $7-4$ shows the beam profile at position $(n e=4) z=7.9$, with the Inline-Injection system of Solenoid + Gun + Solenoid combination inline with the linac. With Solenoid current of $\mathrm{I}=2400 \mathrm{amp}$.

Figure $7-5$ shows the beam profile at position $(n e=5) z=10$, with the Inline-Injection system of Solenoid + Gun + Solenoid combination inline with the linac. With Solenoid current of $I=2400 \mathrm{amp}$.

Figure $7-6$ shows the beam profile at position $(n e=10) z=20$ with the Inline-Injection system of Solenoid + Gun + Solenoid combination inline with the linac. With Solenoid current of $I=2400 \mathrm{amp}$.

Figure 7 -7 shows the beam profile at position $(\mathrm{ne}=20) \mathrm{z}=40$, with the Inline-Injection system of Solenoid + Gun + Solenoid combination inline with the linac. With Solenoid current of $I=2180 \mathrm{amp}$.

Figure 7-8 shows the beam profile at position $(\mathrm{ne}=30) \mathrm{z}=70$, with the Inline-Injection system of Solenoid + Gun + Solenoid combination inline with the linac. With Solenoid current of $I=2400 \mathrm{amp}$.

Figures $8-1$ to $8-10$ shows the beam profile at various positions (distance $z$ from the cathode), with the Inline-Injection system of Solenoid + Gun + Solenoid combination inline with the linac. With Solenoid current of $I=2500$ amp.

Figure $8-1$ shows the beam profile at position $(n e=1) \quad z=0$, with the InlineInjection system of Solenoid + Gun + Solenoid combination inline with the linac. With Solenoid current of $\mathrm{I}=2500 \mathrm{amp}$.

Figure $8-2$ shows the beam profile at position (ne=2) $z=2.6$, with the Inline-Injection system of Solenoid + Gun + Solenoid combination inline with the linac. With Solenoid current of $I=2500 \mathrm{amp}$.

Figure $8-3$ shows the beam profile at position $(n e=3) z=5.2$, with the Inline-Injection system of Solenoid + Gun + Solenoid combination inline with the linac. With Solenoid current of $I=2500 \mathrm{amp}$.

Figure 8-4 shows the beam profile at position $(n e=4) z=7.9$, with the InlineInjection system of Solenoid + Gun + Solenoid combination inline with the linac. With Solenoid current of $\mathrm{I}=2500 \mathrm{amp}$. 
Figure $8-5$ shows the beam profile at position $(n e=5) \quad z=10$, with the InlineInjection system of Solenoid + Gun + Solenoid combination inline with the linac. With Solenoid current of $I=2500 \mathrm{amp}$.

Figure $8-6$ shows the beam profile at position $(n e=10) z=20$ with the Inline-Injection system of Solenoid + Gun + Solenoid combination inline with the linac. With Solenoid current of $I=2500 \mathrm{amp}$.

Figure $8-7$ shows the beam profile at position $(n e=20) z=40$, with the Inline-Injection system of Solenoid + Gun + Solenoid combination inline with the linac. With Solenoid current of $I=2180 \mathrm{amp}$.

Figure $8-8$ shows the beam profile at position $(n e=27) z=58$, with the Inline-Injection system of Solenoid + Gun + Solenoid combination inline with the linac. With Solenoid current of $I=2500 \mathrm{amp}$.

Figure 8 -9 shows the beam profile at position $(n e=30) z=70$, with the Inline-Injection system of Solenoid + Gun + Solenoid combination inline with the linac. With Solenoid current of $\mathrm{I}=2500 \mathrm{amp}$.

Figure $8-10$ shows the beam profile at position $(n e=35) \quad z=95$, with the Inline-Injection system of Solenoid + Gun + Solenoid combination inline with the linac. With Solenoid current of $\mathrm{I}=2500 \mathrm{amp}$.

Figures $9-1$ to 9-9 shows the beam profile at various positions (distance $z$ from the cathode), with the Inline-Injection system of Solenoid + Gun + Solenoid combination inline with the linac. With Solenoid current of $I=2600$ amp.

Figure $9-1$ shows the beam profile at position $(n e=1) z=0$, with the InlineInjection system of Solenoid + Gun + Solenoid combination inline with the linac. With Solenoid current of $I=2600 \mathrm{amp}$.

Figure $9-2$ shows the beam profile at position $(n e=2) z=2.6$, with the Inline-Injection system of Solenoid + Gun + Solenoid combination inline with the linac. With Solenoid current of $I=2600 \mathrm{amp}$.

Figure $9-3$ shows the beam profile at position $(n e=3) z=5.2$, with the Inline-Injection system of Solenoid + Gun + Solenoid combination inline with the linac. With Solenoid current of $I=2600 \mathrm{amp}$.

Figure 9-4 shows the beam profile at position $(n e=4) \quad z=7.9$, with the InlineInjection system of Solenoid + Gun + Solenoid combination inline with the linac. With Solenoid current of $\mathrm{I}=2600 \mathrm{amp}$. 
Figure $9-5$ shows the beam profile at position (ne=5) $z=10$, with the InlineInjection system of Solenoid + Gun + Solenoid combination inline with the linac. With Solenoid current of $\mathrm{I}=2600 \mathrm{amp}$.

Figure $9-6$ shows the beam profile at position $(n e=10) z=20$ with the Inline-Injection system of Solenoid + Gun + Solenoid combination inline with the linac. With Solenoid current of $I=2600 \mathrm{amp}$.

Figure $9-7$ shows the beam profile at position $(n e=20) z=40$, with the Inline-Injection system of Solenoid + Gun + Solenoid combination inline with the linac. With Solenoid current of $I=2600 \mathrm{amp}$.

Figure 9.8 shows the beam profile at position $(n e=30) z=70$, with the Inline-Injection system of Solenoid + Gun + Solenoid combination inline with the linac. With Solenoid current of $\mathrm{I}=2600 \mathrm{amp}$.

Figure 9-9 shows the beam profile at position $(n e=34) z=90$, with the Inline-Injection system of Solenoid + Gun + Solenoid combination inline with the linac. With Solenoid current of $\mathrm{I}=2600 \mathrm{amp}$.

Figures $10-1$ to 10-9 shows the beam profile at various positions (distance $z$ from the cathode), with the Inline-Injection system of Solenoid + Gun + Solenoid combination inline with the linac. With Solenoid current of $I=2800$ amp.

Figure $10-1$ shows the beam profile at position (ne=1) $\mathrm{z}=0$, with the InlineInjection system of Solenoid + Gun + Solenoid combination inline with the linac. With Solenoid current of $I=2800 \mathrm{amp}$.

Figure $10-2$ shows the beam profile at position $(n e=2) z=2.6$, with the Inline-Injection system of Solenoid + Gun + Solenoid combination inline with the linac. With Solenoid current of $I=2800 \mathrm{amp}$.

Figure $10-3$ shows the beam profile at position $(\mathrm{ne}=3) \mathrm{z}=5.2$, with the Inline-Injection system of Solenoid + Gun + Solenoid combination inline with the linac. With Solenoid current of $\mathrm{I}=2800 \mathrm{amp}$.

Figure 10-4 shows the beam profile at position (ne=4) $z=7.9$, with the Inline-Injection system of Solenoid + Gun + Solenoid combination inline with the linac. With Solenoid current of $I=2800 \mathrm{amp}$.

Figure 10 -5 shows the beam profile at position $(n e=5) z=10$, with the Inline-Injection system of Solenoid + Gun + Solenoid combination inline with the linac. With Solenoid current of $I=2800 \mathrm{amp}$. 
Figure $10-6$ shows the beam profile at position (ne=10) $z=20$, with the Inline-Injection system of Solenoid + Gun + Solenoid combination inline with the linac. With Solenoid current of $I=2800 \mathrm{amp}$.

Figure $10-7$ shows the beam profile at position (ne $=20) z=40$, with the Inline-Injection system of Solenoid + Gun + Solenoid combination inline with the linac. With Solenoid current of $I=2800 \mathrm{amp}$.

Figure 10-8 shows the beam profile at position $(\mathrm{ne}=30) \mathrm{z}=70$, with the Inline-Injection system of Solenoid + Gun + Solenoid combination inline with the linac. With Solenoid current of $I=2800 \mathrm{amp}$.

Figure 10-9 shows the beam profile at position $(\mathrm{ne}=34) \mathrm{z}=90$, with the Inline-Injection system of Solenoid + Gun + Solenoid combination inline with the linac. With Solenoid current of $I=2800 \mathrm{amp}$.

Figures $11-1$ to $11-9$ shows the beam profile at various positions (distance $\mathrm{z}$ from the cathode), with the Inline-Injection system of Solenoid + Gun + Solenoid combination inline with the linac. With Solenoid current of $I=3000$ amp.

Figure $11-1$ shows the beam profile at position (ne=1) $z=0$, with the InlineInjection system of Solenoid + Gun + Solenoid combination inline with the linac. With Solenoid current of $I=3000$ amp.

Figure $11-2$ shows the beam profile at position $(n e=2) z=2.6$, with the Inline-Injection system of Solenoid + Gun + Solenoid combination inline with the linac. With Solenoid current of $I=3000 \mathrm{amp}$.

Figure $11-3$ shows the beam profile at position $(n e=3) \quad z=5.2$, with the Inline-Injection system of Solenoid + Gun + Solenoid combination inline with the linac. With Solenoid current of $\mathrm{I}=3000 \mathrm{amp}$.

Figure 11-4 shows the beam profile at position (ne=4) $z=7.9$, with the Inline-Injection system of Solenoid + Gun + Solenoid combination inline with the linac. With Solenoid current of $I=3000 \mathrm{amp}$.

Figure $11-5$ shows the beam profile at position $(n e=5) z=10$, with the Inline-Injection system of Solenoid + Gun + Solenoid combination inline with the linac. With Solenoid curren $\iota$ of $I=3000 \mathrm{amp}$.

Figure $11-6$ shows the beam profile at position $(n e=10) z=20$, with the Inline-Injection system of Solenoid + Gun + Solenoid combination inline with the linac. With Solenoid current of $\mathrm{I}=3000 \mathrm{amp}$. 
Figure $11-7$ shows the beam profile at position $(n e=20) z=40$, with the Inline-Injection system of Solenoid + Gun + Solenoid combination inline with the linac. With Solenoid current of $I=3000 \mathrm{amp}$.

Figure 11-8 shows the beam profile at position (ne $=30) \mathrm{z}=70$, with the Inline-Injection system of Solenoid + Gun + Solenoid combination inline with the linac. With Solenoid current of $\mathrm{I}=3000 \mathrm{amp}$.

Figure 11-9 shows the beam profile at position $(n e=34) \quad z=90$, with the Inline-Injection system of Solenoid + Gun + Solenoid combination inline with the linac. With Solenoid current of $I=3000 \mathrm{amp}$.

Figures $12-1$ to $12-10$ shows the beam profile at various positions (distance $\mathrm{z}$ from the cathode), with the Inline-Injection system of Solenoid + Gun + Solenoid combination inline with the linac. With Solenoid current of $I=3400$ amp.

Figure $12-1$ shows the beam profile at position $(n e=1) \quad z=0$, with the InlineInjection system of Solenoid + Gun + Solenoid combination inline with the linac. With Solenoid current of $\mathrm{I}=3400 \mathrm{amp}$.

Figure $12-2$ shows the beam profile at position (ne=2) $\mathrm{z}=2.6$, with the Inline-Injection system of Solenoid + Gun + Solenoid combination inline with the linac. With Solenoid current of $I=3400 \mathrm{amp}$.

Figure $12-3$ shows the beam profile at position $(n e=3) z=5.2$, with the Inline-Injection system of Solenoid + Gun + Solenoid combination inline with the linac. With Solenoid current of $I=3400 \mathrm{amp}$.

Figure 12-4 shows the beam profile at position $(\mathrm{ne}=4) \mathrm{z}=7.9$, with the Inline-Injection system of Solenoid + Gun + Solenoid combination inline with the linac. With Solenoid current of I=3400 amp.

Figure $12-5$ shows the beam profile at position $(n e=5) z=10$, with the Inline-Injection system of Solenoid + Gun + Solenoid combination inline with the linac. With Solenoid current of $I=3400 \mathrm{amp}$.

Figure $12-6$ shows the beam profile at position $(n e=10) \quad z=20$, with the Inline-Injection system of Solenoid + Gun + Solenoid combination inline with the linac. With Solenoid current of I=3400 amp.'

Figure $12-7$ shows the beam profile at position (ne=20) $z=40$, with the Inline-Injection system of Solenoid + Gun + Solenoid combination inline with the linac. With Solenoid current of $I=3400 \mathrm{amp}$. 
Figure 12-8 shows the beam profile at position $(n e=27) z=58$, with the Inline-Injection system of Solenoid + Gun + Solenoid combination inline with the linac. With Solenoid current of $I=3400 \mathrm{amp}$.

Figure 12-9 shows the beam profile at position $(n e=30) z=70$, with the Inline-Injection system of Solenoid + Gun + Solenoid combination inline with the linac. With Solenoid current of $I=3400 \mathrm{amp}$.

Figure 12-10 shows the beam profile at position (ne $=35) \quad z=95$, with the Inline-Injection system of Solenoid + Gun + Solenoid combination inline with the linac. With Solenoid current of $\mathrm{I}=3400 \mathrm{amp}$.

Figures $13-1$ to 13-10 shows the beam profile at various positions (distance $\mathrm{z}$ from the cathode), with the Inline-Injection system of Solenoid + Gun + Solenoid combination inline with the linac. With Solenoid current of $I=3800$ amp.

Figure $13-1$ shows the beam profile at position (ne=1) $z=0$, with the InlineInjection system of Solenoid + Gun + Solenoid combination inline with the linac. With Solenoid current of $\mathrm{I}=3800 \mathrm{amp}$.

Figure $13-2$ shows the beam profile at position $(n e=2) z=2.6$, with the Inline-Injection system of Solenoid + Gun + Solenoid combination inline with the linac. With Solenoid current of $I=3800 \mathrm{amp}$.

Figure $13-3$ shows the beam profile at position $(n e=3) \quad z=5.2$, with the Inline-Injection system of Solenoid + Gun + Solenoid combination inline with the linac. With Solenoid current of $I=3800$ amp.

Figure 13-4 shows the beam profile at position $(\mathrm{ne}=4) \mathrm{z}=7.9$, with the Inline-Injection system of Solenoid + Gun + Solenoid combination inline with the linac. With Solenoid current of $\mathrm{I}=3800 \mathrm{amp}$.

Figure $13-5$ shows the beam profile at position $(n e=5) z=10$, with the Inline-Injection system of Solenoid + Gun + Solenoid combination inline with the linac. With Solenoid current of $\mathrm{I}=3800 \mathrm{amp}$.

Figure $13-6$ shows the beam profile at position $(n e=10) \quad z=20$, with the Inline-Injection system of Solenoid + Gun + Solenoid combination inline with the linac. With Solenoid current of $\mathrm{I}=3800 \mathrm{amp}$.

Figure $13-7$ shows the beam profile at position $(n e=20) z=40$, with the Inline-Injection system of Solenoid + Gun + Solenoid combination inline with the linac. With Solenoid current of $\mathrm{I}=3800 \mathrm{amp}$. 
Figure 13-8 shows the beam profile at position (ne=27) $z=58$, with the Inline-Injection system of Solenoid + Gun + Solenoid combination inline with the linac. With Solenoid current of $\mathrm{I}=3800 \mathrm{amp}$.

Figure 13-9 shows the beam profile at position $(\mathrm{ne}=30) \mathrm{z}=70$, with the Inline-Injection system of Solenoid + Gun + Solenoid combination inline with the linac. With Solenoid current of $\mathrm{I}=3800 \mathrm{amp}$.

Figure 13-10 shows the beam profile at position $(n e=35) \quad z=95$, with the Inline-Injection system of Solenoid + Gun + Solenoid combination inline with the linac. With Solenoid current of $\mathrm{I}=3800 \mathrm{amp}$. 


\section{REFERENCES}

-Work performed under the auspices of U.S. Dept.of Energy contract DE-ACO2-T6-CH00016.

1. Z. Parsa, "Preliminary Emittance Measurements and Calculations of the Laser Excited Beam from the BNL Accelerator Test Facility Radio Frequency Electron Gun", Vol.II, Sec.II, pI-54, BNL-52292 (1991) and References therein; K. McDonald et al., Proc., EPAC88, June 1988; A. Fisher, J. Fisher, I. pogorelsky, private communications on laser performance.;

Z. Parsa, "Effects of 3rd Order Maps and Solenoid on a Eigh Brightness Beam", BNL-4839i (1993) and Proc. IEEE PAC May 17-20, Washington D.C. (1993).

2. K. Batehelor, I. Ben-Zvi, I. Bigio, T.S. Chou, R.C. Fernow, J. Fischer, J. Gallardo, E. Kirk, N. Kurnit: K.T. McDonald, R.B. Palmer, Z. Parsa, C. Pellegrini, J. Sheehan, T. Srinivasan-Rao, S. Ule, A. Van Steenbergen, M. Woodle, "Operational Status of Brookhaven Accelerator Test Facility “, Proc. March 20-23:1989 IEEE PAC, Chicago: 기, \#89CH2669-0 (1990).

3. 2. Parsa, "On Beam Emittance and Invariants - Applications to ATF Beamline": Proc.2nd EPAC, ViceFrance, Nile Ed., Frontiers, (1990); BNL Report BNL44364 (1990) and Ref.therein.

4. Z. Parsa, and L. Young, "Modeling of the BNL Photocathode Gun with the Code PAR.MELA": Nucl. Inst. \& . Meths. 1318 (1992) 254258.

5. L. Young, (Private comms.), PAR.VIELA, 1993 version: We thank L. Young for helpful discussions: modifications to the programs and collaborations.

6. Z. Parsa, "An Alternate Injection System for the B:VL Accelerator Test Facility", BNL-48403, APS April 1215, Washington D.C. (1993); Z. Parsa, "Intercetions Between Charged Particles and RF Space Charge and Wake Fields in an Accelerating Structure": Particles and Fields 91, Vol. 2, 1073, Ed. David Axen. Douglas Bayman and Martin Comyn; $Z$. Parsa. Accelerator Physics and Modeling, BNL-Report BNL-52379, 65165 (1993). 